\title{
New Potent Human Acetylcholinesterase Inhibitors in the Tetracyclic Triterpene series with Inhibitory Potency on Amyloid $\beta$ Aggregation
}

\author{
Julien Rouleau, Bogdan Iorga and Catherine Guillou* \\ Institut de Chimie des Substances Naturelles, Bt 27, CNRS Avenue de la Terrasse \\ 91198 Gif-sur-Yvette, France

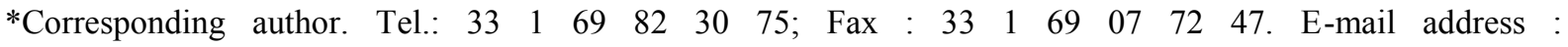 \\ catherine.guillou@icsn.cnrs-gif.fr

\begin{abstract}
New acetylcholinesterase inhibitors in the tetracyclic triterpene series were synthesized, tested in vitro for the inhibition of cholinesterases (different sources of $\mathrm{AChE}$ and $\mathrm{BuChE}$ ) and for the ability to prevent $\mathrm{AChE}$-induced $\mathrm{A} \beta$ aggregation. Some compounds have $h \mathrm{AChE} \mathrm{IC}_{50}$ values in the nanomolar range and showed ability to block the AChE-induced $\mathrm{A} \beta$ aggregation. The mode of interaction between EeAChE and compounds $\mathbf{1}$ and 36e was investigated using docking and molecular dynamics simulations. These studies suggested that both compounds interact simultaneously with the catalytic and the peripheral sites of AChE, and the nature of protein-ligand interactions is mainly hydrophobic.
\end{abstract}

\section{Keywords}

Acetylcholinesterase inhibitors; N-3-isobutyrylcycloxobuxidine-F derivatives; Molecular modeling; Alzheimer's disease; $\beta$-amyloid aggregation; Butyrylcholinesterase; Synthesis.

\section{Introduction}

Alzheimer disease (AD) is a progressive neurodegenerative disorder of the central nervous system (CNS) that affects mainly aged population. AD is characterized by profound memory impairments, emotional disturbance, and also personality changes. The main pathological changes in the $\mathrm{AD}$ brain are extracellular amyloid plaques [1], intracellular neurofibrillary tangles containing abnormally hyperphosphorylated tau protein [2], and loss of neurons in the nucleus basalis of Meynert and the hippocampus. AD is characterized by a pronounced 
alteration of the cholinergic system and other neurotransmitter systems (glutamate and serotonine). The cholinergic hypothesis postulates that memory impairments in patients with $\mathrm{AD}$ result from a deficit of cholinergic function in the brain [3]. Most currently prescribed AD drugs aim to increase the level of acetylcholine (ACh) in the brain by inhibiting $\mathrm{AChE}$. However, clinical use of AChE inhibitors is sometimes limited mainly due to their adverse effects and modest benefits to AD patients. Therefore, novel more effective therapies, including $\mathrm{AChE}$ inhibitors, need to be developed. In addition to its catalytic activity, $\mathrm{AChE}$ exerts secondary noncholinergic functions related to its peripheral binding site on differentiation, cell adhesion, in mediating the processing and deposition of $\beta$-amyloid peptide $(\mathrm{A} \beta)$ [2]. It was postulated that $\mathrm{AChE}$ binds through its peripheral site to the non amyloidogenic form of $\beta$-amyloid protein acting as a chaperone protein and inducing conformational change to the amyloidogenic form with the subsequent amyloid fibril formation. Moreover, it has been shown that molecules which are able to interact with both the active and peripheral sites of AChE could prevent the aggregating activity of AChE towards $\mathrm{A} \beta$ besides the inhibitory activity $[3,4,5]$. Therefore, inhibitors with dual binding to AChE represent a new therapeutic strategic option [6,7].

In continuation of our on-going research on new AChE inhibitors [8], we will present our recent studies on acetylcholinesterase inhibitor in the tetracyclic triterpene series. We previously reported that compound $\mathbf{1}$ was identified by high-throughput screening of The Institut de Chimie des Substances Naturelles' chemical library. Some hemisynthetic analogs of $\mathbf{1}$ (i.e. $\mathbf{2 a}$ and $\mathbf{2 b}$ ) were found to be very active on $E e \mathrm{AChE}$ and $T c \mathrm{AChE}$ but less potent on $h \mathrm{AChE}$ (Figure 1). The aim of the present study is to develop new potent $h \mathrm{AChE}$ inhibitors in this series. 
Herein we describe the synthesis, pharmacological evaluations and molecular modeling of a novel class of analogs.

These new inhibitors contain a modified heterocycle which is supposed to interact with the catalytic site and new substituents connected to the nitrogen atom N 20, which is supposed to be responsible for the binding to the peripheral site of the enzyme.

Newly synthesized compounds were tested in vitro for $\mathrm{AChE}$ and $\mathrm{BuChE}$ inhibition potency. Their selectivity for $h$ AChE was also evaluated. The ability of compounds $\mathbf{3 6 e}, \mathbf{3 6 f}$, 36i, and 36k to inhibit the AChE-induced $\mathrm{A} \beta$ aggregation compared with propidium iodide and tacrine was assayed by means of a thioflavin T-based fluorometric assay [9]. A molecular modeling study (docking and molecular dynamics simulations) of $\mathbf{1}$ and 36e was also performed on EeAChE.

\section{[Figure 1.]}

\section{Chemistry}

Introduction of fluorine atoms on a molecule can modify its properties [10]. In addition, the presence of a triflurometyl group on $m-(\mathrm{N}, \mathrm{N}, \mathrm{N},-$ trimethylammonio) trifluoroacetophenone (TMTFA) or zifrosilone greatly improved their acetylcholinesterase inhibition potency [11]. Replacement of the isopropyl group present in the 1' position of the tritepene tetracyclic scaffold of 1 by other substituents bearing one, three or seven fluorine atoms was first envisaged. The new fluoro analogs 9a-9c were synthesized from cyclobuxidine-F 4 obtained by hydrolysis of $\mathrm{N}$-3-isobutyryl-cycloxobuxidine-F 3 (Scheme 1) [12]. Preparation of amides 6a-c was carried out by coupling trifluoroacetic anhydride or 2-fluoro-2-methylpropanoic acid or methyl heptafluoroisobutyrate with cycloxobuxidine-F 4. The 16-hydroxyfunction was first protected as an acetate (i.e. $\mathbf{7 a - b}$ ) or as a benzoate (i.e. 7c). The nitrogen atom at position 20 was protected as a salt by reaction with APTS before the transformation of the alcohol group 
at position 29 to the corresponding tosylates (i.e. 8a-c). The acetate and benzoate protective groups were then removed under basic conditions. Finally, treatment of the resulting tosylates with sodium diformylamide in DMF provided the expected fluoro oxazines 9a-c (Scheme 1).

\section{[Scheme 1.]}

The N-substituted tetrahydropyrimidines derivatives $\mathbf{1 3 a} \mathbf{a}-\mathbf{b}$ were synthesized following the reactions depicted in Scheme 2 using the aldehyde 10 [8] as key intermediate, which was converted into the corresponding amines 12a-b by aminoreduction. Subsequent heating under microwaves irradiation furnished 13a-b (Scheme 2).

\section{[Scheme 2.]}

The new dihydro-1H-imidazole analog $\mathbf{2 0}$ was synthesized from N-3isobutyrylcycloxobuxidine-F 3. The 16-hydroxy function was first protected as a benzoyl (i.e. 14). Oxidation of the alcohol group at position 29 with Jones' reagent afforded the acid (i.e. 15). Reaction of $\mathbf{1 5}$ with oxalyl chloride provided the corresponding acid chloride which reacted with sodium azide to give the carbonyl azide 16. Curtius rearrangement of $\mathbf{1 6}$ under microwaves irradiation furnished the isocyanate 17. Subsequent hydrolysis with lithium hydroxide, heating in methanol, and treatment under acid conditions provided a 90/10 mixture diamine 18 (major) and the amino amide 19 (minor). Finally, the dihydro- $1 H$-imidazole 20 was prepared by reaction of $\mathbf{1 8}$ and methyl orthoisobutyrate in the presence of ytterbium triflate (Scheme 3).

\section{[Scheme 3.]}

The dihydropyrimidine 25 was synthesized from cycloxobuxidine -F 4 . First, the primary amine was protected with tert-butyl dicarbonate and the 16-hydroxy function was transformed into a benzoate (i.e. 22). Oxidation of the alcohol group at position 29 with Dess-Martin periodinane afforded the corresponding aldehyde 23. Aldolisation of $\mathbf{2 3}$ with 3-methyl-2- 
butanone led to the keto alcohol $\mathbf{2 4}$. Subsequent cyclization under acid conditions followed by deprotection of the benzoate group furnished the expected dihydropyridine $\mathbf{2 5}$ (Scheme 4).

\section{[Scheme 4.]}

The tetrahydropyridine $\mathbf{2 7}$ was synthesized from the keto alcohol $\mathbf{2 4}$. Dehydratation of 24 was first performed using potassium carbonate to afford the $\alpha, \beta$-unsaturated ketone 26 . The tetrahydropyridine $\mathbf{2 7}$ was obtained after hydrogenation of $\mathbf{2 6}$ over palladium hydroxide and cyclization under acid conditions in the presence of trifluoroacetic acid (Scheme 5).

\section{[Scheme 5. ]}

The synthesis of compound $\mathbf{3 0}$ was achieved following the strategy summarized in scheme 6. Reaction of the known tosylate 28 [13] with potassium thioacetate afforded a thioacetate intermediate that was directly hydrolyzed under basic conditions to provide the thiol 29 . The 5,6-dihydro-4H-1,3-thiazine $\mathbf{3 0}$ was obtained by cyclization of $\mathbf{2 9}$ in the presence of trimethylaluminium (Scheme 6).

\section{[Scheme 6. ]}

Synthetic routes to $N$-substituted thiazine analogs $35 \mathbf{a}-\mathbf{b}$ and carbamate $35 \mathbf{c}$ were also developed. The alcohol group at position 29 of $\mathbf{1 4}$ was transformed into the corresponding tosylate (i.e. 31) and then substituted with potassium thioacetate to give the corresponding thioacetate. This later was converted into its $N$-oxide by oxidation with $m$-chloroperbenzoic acid. Subsequent treatment of this $N$-oxide with a mixture of ferrous sulfate heptahydrate and iron chloride hexahydrate provided $\mathrm{N}$-demethylated amine 32. Hydrolysis of the thioacetate 32 provided the thiol $\mathbf{3 3}$ which was cyclized in the presence of trimethylaluminium (i.e. $\mathbf{3 4}$ ). Acylation or alkylation of the amine $\mathbf{3 4}$ furnished the expected amides 35a-c and the tertiary amines 36a-k (Scheme 7). 


\section{[Scheme 7.]}

\section{Results and Discussion}

\subsection{AChE and BuChE Inhibitory Activity and AChE/BuChE Selectivity}

To determine the potential interest of the newly synthesized compounds for the treatment of $\mathrm{AD}$, their AChE inhibitory activity was assayed by the method of Ellman [14] using Electrophorus electricus and human recombinant $\mathrm{AChE}$ and tacrine, $\mathbf{1}$ and $\mathbf{3}$ as reference compounds. Moreover, to further study the biological profiles of the novel compounds, their butyrylcholinesterase $(\mathrm{BuChE})$ inhibitory activity on human serum butyrylcholinesterase ( $h \mathrm{BuChE})$ was also determined by the same method. Recent studies have shown that in AD patients with severe pathology, BuChE increases while $\mathrm{AChE}$ is reduced in specific brain regions [15] this is not in agreement with a recent study which shows a decrease of BuChE activity in vivo [16]. The results are summarized in Table 1.

\section{[Table 1]}

The inhibitory potency depends on numerous factors. Introduction of fluoro groups at position 1' of the oxazine scaffold (i.e. 9a-9c) decreased the inhibitory activity for AChE and BuChE.

Replacement of the oxazine ring by different heterocycle rings affected the affinity for $E e \mathrm{AChE}$ and $h \mathrm{AChE}$. An order of the inhibitory potency for AChE can be established among the various heterocycles : alkylated tetrahydropyrimidines (i.e 13a-b) $\approx$ imidazole (i.e. $\mathbf{2 0}$ ) < tetrahydropyrimidine (i.e. 27) $<$ thiazine (i.e. 30) $<$ dihydropyrimidine (i.e. 25). The dihydropyrimidine $\mathbf{2 5}$ is the most potent inhibitor in these series (12 fold more active than $\mathbf{1}$ ) and selective for $h \mathrm{AChE}$. Compounds 13a-b and $\mathbf{2 0}$ are more potent on BuChE than on AChE. 
The analogs of second generation with modified nitrogen atom at position 20 were also evaluated. This series was envisaged in order to increase the binding of the inhibitors with the peripheral site of $\mathrm{AChE}$ and thereby increasing the inhibitory potency.

$\mathrm{N}$-demethylation of the nitrogen atom of the thiazine (i.e 34, $h \mathrm{AChE} \mathrm{IC}_{50}=76 \mathrm{nM}$ ) produced no significant change on $\mathrm{AChE}$ inhibition (i.e 30, $h \mathrm{AChE} \mathrm{IC}_{50}=60 \mathrm{nM}$ ) but decreased the selectivity for AChE (i.e 34, 17; i.e 30, 89).

In the amide series (i.e 35a-35c) introduction of a phenyl group (i.e. 35b) increased the inhibition activity likely as a result of a better interaction with the peripheral site of the enzyme probably via hydrophobic interactions. We previously reported that compound $\mathbf{1}$ was able to bind simultaneously to both catalytic and peripheral sites of AChE. Its oxazine scaffold interacted with the active site and the nitrogen atom at position 20 with the peripheral site [8].

The AChE inhibitory potency within the $\mathrm{N}$-alkylated series of thiazine derivatives is related to the substituent, to the substitution of $\mathrm{R}$ and to the length of the linker between $\mathrm{R}$ and the nitrogen atom. The inhibition activity follows this order : $\left(\mathrm{CH}_{2}\right)_{2} \mathrm{Bn}$ (i.e. 36c) $<\left(\mathrm{CH}_{2}\right)_{2} \mathrm{Pht}$ (i.e. 36j) $<\mathrm{CH}_{2} \mathrm{Bn}\left(\right.$ i.e. 36b) $<\left(\mathrm{CH}_{2}\right)_{3}$ Pht (i.e. 36k) $<o-\mathrm{CF}_{3} \mathrm{Bn}($ i.e. 36g) $<p-\mathrm{MeOBn}($ i.e. 36f) $<$ Bn (i.e. 36a) $\approx o-\mathrm{MeOBn}\left(\right.$ i.e. 36d) $<p-\mathrm{CF}_{3} \mathrm{Bn}\left(\right.$ i.e. 36h) $\approx m-\mathrm{CF}_{3} \mathrm{Bn}($ i.e. 36h) $\approx m-\mathrm{MeOBn}$ (i.e. 36e). Introduction of a $\mathrm{CF}_{3}$ substituent on the benzyl ring increased the selectivity for $h \mathrm{AChE}$ versus $\mathrm{BuChE}$.

\subsection{Molecular Modeling Studies}

In order to gain insight into the mode of interaction between this new class of inhibitors and AChE, molecular modeling studies were performed using the compound $\mathbf{3 6 e}$, the most potent in the series, as a representative member. The results were compared with those obtained for the apo AChE and for the complex with oxazine 1. 
Molecular docking calculations showed that compound 36e is positioned in a conformation similar to those proposed previously for oxazine 1 [8], and that it is able to interact with both the catalytic and peripheral sites of AChE. The binding site was defined as a sphere with a radius of $20 \AA$ around the $\mathrm{OH}$ group of SER200 and the protein was kept rigid during the docking, whereas the ligand was fully flexible. No hydrogen bond could be identified between oxazine 1 and the protein, and only one was present in the AChE-36e complex, between $\mathrm{OH}$ group in position 16 and $\mathrm{OH}$ group of TYR70. Furthermore, in order to take into account the protein flexibility and a possible induced-fit that could take place upon ligand binding, molecular dynamics simulations were carried out, using the docking results as starting conformations.

Three molecular dynamics simulations, of $10 \mathrm{~ns}$ each, were set up, one with the apo protein (Sim1), for comparison purposes, and the other two with oxazine 1 (Sim2) and compound 36e (Sim3). All three structures were generally stable during the simulation, the root mean square deviation (RMSD) for protein reaching a plateau in less than $5 \mathrm{~ns}$, at $1.8 \AA$ for $\operatorname{Sim} 3$ and at about $2 \AA$ for $\operatorname{Sim} 1$ and $\operatorname{Sim} 2$. In $\operatorname{Sim} 2$ and $\operatorname{Sim} 3$, the ligands attained very quickly an equilibrium state with a RMSD of $0.7 \AA$, which was conserved throughout the simulation (Figure S1, Supporting Information). All simulations showed three main regions with higher flexibility (around residues 73, 341 and 378), but none of them were in the binding site region (Figure S2, Supporting Information).

Generally speaking, very few protein-ligand hydrogen bonds were identified in Sim2 and Sim3, the interactions being mostly hydrophobic. In Sim2, only the interaction between the $\mathrm{OH}$ group in position 16 of the steroid skeleton and the $\mathrm{OH}$ group of TYR70 was scarcely observed during the simulation (less than $10 \%$ of the simulation time). In Sim3, three hydrogen bonds were present all along the simulation : i) the same interaction mentioned above, for about $50 \%$ of the simulation time; ii) a hydrogen bond between the $\mathrm{OH}$ group in 
position 16 of compound 36e and the carboxyl group of ASP72, present all the time; iii) an interaction between the oxygen in position 11 of steroid and the $\mathrm{OH}$ group of TYR121, present for about $25 \%$ of the simulation time. Therefore, it seems that compound 36e can establish better interactions than oxazine 1 within the binding site of AChE and these interactions may be responsible, at least in part, for the good inhibition observed for the compound 36e.

The evolution of the protein-ligand contact surface during the simulations $\operatorname{Sim} 2$ and $\operatorname{Sim} 3$ is shown in Figure S3 (Supporting Information). Whereas during the first 5 ns the surfaces are rather comparable, during the last $5 \mathrm{~ns}$ the protein interaction with compound 36e is clearly more favored than those with oxazine 1. A possible explanation for this behavior may be that AChE better accommodates the cyclopropyl-derived scaffold of compound 36e than the seven-membered ring-derived core of oxazine $\mathbf{1}$. This result is also in agreement with the lower RMSD observed for protein in Sim3 (see above) and possibly could explain the good biologicaly activity measured experimentally for compound 36e.

In the apo simulation ( $\operatorname{Sim} 1)$, the residues forming the binding site did not show important movements, with the exception of TRP84, which underwent a $3.6 \AA$ movement in the direction of the binding site opening, and of the TYR334-PHE330-PHE331 triplet, which underwent a concerted movement resulting in the complete closure of the gorge (Figure 2). Thus, it seems that in the absence of a ligand this closed conformation is more stable than the open conformation originally present in the X-ray structure.

\section{[Figure 2.]}

\section{[Figure 3.]}

In both $\operatorname{Sim} 2$ and $\operatorname{Sim} 3$ simulations, the ligands move about $2.8 \AA$ towards the peripheral site (Figures 3 and 4), to reach a stable, common equilibrium position (Figure S4, Supporting Information). In the meantime, the residues surrounding the ligand move apart in order to 
accommodate its presence and to maximize the hydrophobic interactions. Unexpectedly, no cation- $\pi$ and $\pi-\pi$ interactions could be evidenced between the TRP279 at the peripheral site and the positively charged nitrogen in position 20 and/or its aromatic substituent (in the case of compound 36e).

\section{[Figure 4.]}

\subsection{Inhibition of AChE-Induced A Aggregation}

Three compounds, $\mathbf{3 6 e}, \mathbf{3 6}$ and $\mathbf{3 6 k}$, were selected to assess their abilities to inhibit $\mathrm{A} \beta$ aggregation induced by $\mathrm{AChE}$ using a thioflavin T-based fluorometric assay [9], compared with the reference compound propidium iodide [9], a known specific peripheral site-binding inhibitor and tacrine a known specific inhibitor of the active site [9]. The $\mathrm{N}$ substituted thiazines 36e, 36h and 36k inhibit, at $100 \mu \mathrm{M}$, the AChE-induced $\mathrm{A} \beta$ aggregation with percentages of inhibition ranging from $42 \%$ to $69 \%$ (Table 2). By observation of the data reported in Table 2, it appears that introduction of a meta $\mathrm{CF}_{3}$ group on the $\mathrm{N}$ benzyl terminal group (i.e 36h) or a phtalimide terminal group (i.e. 36k) decrease the anti aggregating action. In contrast, introduction of a $m-\mathrm{OMeBn}$ (i.e. 36e) favors the anti aggregating action. The best compound 36e allows a better positioning of the methoxy aromatic ring with the peripheral site of the enzyme.

Thiazines 36e could be classified as good inhibitor of the AChE-induced $\mathrm{A} \beta$ aggregation. The most potent described inhibitors inhibit, at $100 \mu \mathrm{M}$, the AChE-induced A $\beta$ aggregation with percentages of inhibition ranging from $82 \%$ to $98 \%[17,18,19,20,21]$.

\section{[Table 2.]}

\section{Conclusion}

We have synthesized new inhibitors in the tetracyclic triterpene series. These compounds have been synthesized from the natural product N-3-isobutyrylclyclobuxidine-F. Variations of 
the heterocycle led us to identify that presence of a thiazine or a dihydropyrimidine ring enhanced the $h \mathrm{AChE}$ inhibitory potency. Alkylation of the nitrogen atom at position 20 with functionalized benzyl groups in the thiazine series also increased the $h \mathrm{AChE}$ inhibitory activity. Molecular modeling studies showed that $\mathbf{1}$ and 36e interact simultaneously with both the catalytic and peripheral sites of $E e A C h E$, the nature of protein-ligand interactions being mainly hydrophobic. Futhermore, 36e significantly prevented the AChE-induced $\mathrm{A} \beta$ aggregation. Taken together, all the reported results suggested that new dual binding AChE inhibitors in the thiazine tetracyclic triterpene series are promising leads for the development of disease-modifying drugs for the future treatment of $\mathrm{AD}$.

\section{Experimental section}

\subsection{Chemistry}

All commercially available reagents were used without further purification. All experiments involving water-sensitive compounds were carried out under argon and scrupulously dry conditions, using anhydrous solvents. $\mathrm{MeOH}$ was distilled from $\mathrm{Mg} / \mathrm{I}_{2}$, $\mathrm{CH}_{2} \mathrm{Cl}_{2}$ from $\mathrm{P}_{2} \mathrm{O}_{5}, 1$,4-dioxane from $\mathrm{LiAlH}_{4}, \mathrm{NEt}_{3}$ and pyridine from $\mathrm{KOH}$. All separations were carried out under flash chromatographic conditions on Merck silica gel 60 (70-230 mesh) at medium pressure (200 mbar). TLC was performed on Merck silica gel plates $\left(60 \mathrm{~F}_{254}\right)$ with a fluorescent indicator. NMR spectra were determined on Bruker Avance-300, Bruker AC-400, Bruker AC-500 or Bruker-600 instruments and using tetramethylsilane (TMS) as reference. Chemical shifts are reported in parts per million (ppm) relative to TMS. High resolution mass spectra (HRMS) were obtained on a maldi-toff spectrometer. The assignement of the ${ }^{1} \mathrm{H}$ and ${ }^{13} \mathrm{C}$ NMR spectra of the different compounds has been made by ${ }^{1} \mathrm{H}-{ }^{1} \mathrm{H},{ }^{1} \mathrm{H}-{ }^{13} \mathrm{C}$, COSY, HMBC, HMQC and by analogy with previously described compounds. The numerotation indicated on molecule is only mentioned for the NMR spectra interpretation. Infrared (IR) spectra were recorded on a Fourier Perkin-Elmer Spectrum BX 
FT-IR instruments. Elemental analyses were performed by the microanalysis laboratory of the ICSN, CNRS, Gif-sur-Yvette.

5.1.1. N-[20S-(dimethylamino)-16 $\alpha$-hydroxy-4 $\alpha$-(hydroxymethyl)-4 $\beta, 14 \alpha$-dimethyl-11-oxo-

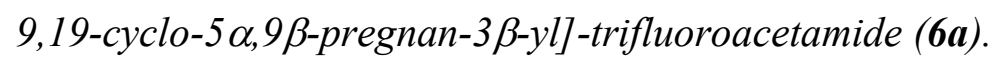

To $68 \mathrm{mg}(0.158 \mathrm{mmol}, 1$ eq. $)$ of cycloxobuxidine-F 3 solubilised in DCM/pyridine (mixture 3/1, $4 \mathrm{~mL}$ ) were added at $0{ }^{\circ} \mathrm{C} 25 \mu \mathrm{L}(0.174 \mathrm{mmol}, 1.1$ eq.) of trifluoroacetic anhydride. The mixture was stirred at $0{ }^{\circ} \mathrm{C}$ for $1 \mathrm{~h}$ then at $\mathrm{RT}$ for further $12 \mathrm{~h}$. After 3 coevaporations with $3 \times 10 \mathrm{~mL}$ of 1,2-dichloroethane, the residue was dissolved in DCM, washed with $20 \mathrm{~mL}$ of a saturated solution of sodium hydrogen carbonate then extracted with $3 \times 20$ $\mathrm{mL}$ of DCM. Combined organic layers were washed with brine $(20 \mathrm{~mL})$, dried over magnesium sulfate and concentrated under vacuum. The crude product was purified by silica gel column chromatography (eluent : $\mathrm{DCM} / \mathrm{MeOH} / \mathrm{NH}_{4} \mathrm{OH}: 96 / 3 / 1$ ) to afford the title compound as an amorphous white solid (70 mg, yield $84 \%$ ). IR (cm¹) : 3276, 2944, 2872, 1698, 1698, 1555, 1454, 1157, 1187, $1209 ;{ }^{1} \mathrm{H}$ NMR (300 MHz, $\left.\mathrm{CDCl}_{3}\right): \delta(\mathrm{ppm}) 6.43(1 \mathrm{H}, \mathrm{d}$, $\left.\mathrm{J}_{\mathrm{NAH}-\mathrm{H} 3}=9.2 \mathrm{~Hz}, \mathrm{~N}_{\mathrm{A}}-\mathrm{H}\right), 4.16(1 \mathrm{H}, \mathrm{m}, \mathrm{H} 16 \beta), 4.12(1 \mathrm{H}, \mathrm{m}, \mathrm{H} 3 \alpha), 3.42\left(1 \mathrm{H}, \mathrm{d}, \mathrm{J}_{\mathrm{gem}}=12.9 \mathrm{~Hz}\right.$, $\mathrm{H} 29 \mathrm{~b}), 3.03\left(1 \mathrm{H}, \mathrm{d}, \mathrm{J}_{\mathrm{gem}}=12.9 \mathrm{~Hz}, \mathrm{H} 29 \mathrm{a}\right), 2.70\left(1 \mathrm{H}, \mathrm{dq}, \mathrm{J}_{20-17 \alpha}=10.8 \mathrm{~Hz}, \mathrm{~J}_{20-21}=6.7 \mathrm{~Hz}\right.$, H20), $2.54\left(1 \mathrm{H}, \mathrm{d}, \mathrm{J}_{\mathrm{gem}}=17.2 \mathrm{~Hz}, \mathrm{H} 12 \alpha\right), 2.50\left(1 \mathrm{H}, \mathrm{ddd}, \mathrm{J}_{\mathrm{gem}}=13.2 \mathrm{~Hz}, \mathrm{~J}_{1 \beta-2}=3.6 \mathrm{~Hz}, \mathrm{~J}_{1 \beta-2}=\right.$ 3.4 Hz, H1 $\beta), 2.32\left(1 \mathrm{H}, \mathrm{d}, \mathrm{J}_{\text {gem }}=17.2 \mathrm{~Hz}, \mathrm{H} 12 \beta\right), 2.31\left(6 \mathrm{H}, \mathrm{sl}, \mathrm{N}_{\mathrm{B}} \mathrm{Me}_{2}\right), 2.10\left(1 \mathrm{H}, \mathrm{dd}, \mathrm{J}_{5 \alpha-6 \beta}=\right.$ $\left.12.5 \mathrm{~Hz}, \mathrm{~J}_{5 \alpha-6 \alpha}=3.6 \mathrm{~Hz}, \mathrm{H} 5 \alpha\right), 2.01(3 \mathrm{H}, \mathrm{m}, \mathrm{H} 17 \alpha+\mathrm{H} 15 \beta+\mathrm{H} 8 \beta), 1.80(1 \mathrm{H}, \mathrm{m}, \mathrm{H} 2 \alpha), 1.71$

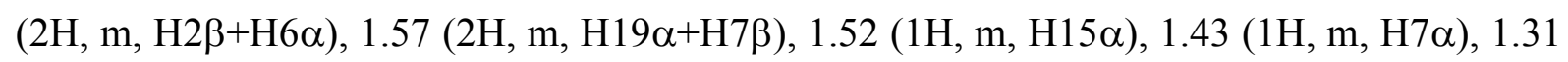
$(1 \mathrm{H}, \mathrm{m}, \mathrm{H} 1 \alpha), 1.22(3 \mathrm{H}, \mathrm{s}, \mathrm{H} 28), 1.06\left(1 \mathrm{H}, \mathrm{d}, \mathrm{J}_{\mathrm{gem}}=3.8 \mathrm{~Hz}, \mathrm{H} 19 \beta\right), 0.92\left(4 \mathrm{H}, \mathrm{m}, \mathrm{J}_{21-20}=\right.$ $6.6 \mathrm{~Hz}, \mathrm{H} 21+\mathrm{H} 6 \beta), 0.86(3 \mathrm{H}, \mathrm{s}, \mathrm{H} 18), 0.64(3 \mathrm{H}, \mathrm{s}, \mathrm{H} 30) ;{ }^{13} \mathrm{C} \mathrm{NMR}\left(75.4 \mathrm{MHz}, \mathrm{CDCl}_{3}\right): \delta(\mathrm{ppm})$ 211.3 (C11), 158.0 (C1'), 113.9 (C2'), 78.0 (C16), 64.2 (C29), 62.4 (C20), 55.8 (C17), 52.4 (C3), 51.4 (C12), 47.1 (C14), 44.6 (C4+C13), 43.1 (C15), 41.5 (C8), 41.2 (C5), 37.2 (C10), 34.3 (C9), 30.5 (C19), 27.4 (C1), 27.2 (C2), 24.3 (C7), 20.7 (C28), 18.4 (C6), 17.9 (C18), 
$11.2(\mathrm{C} 30), 10.1(\mathrm{C} 21)$. MS (ESI, m/z) : $529.2(\mathrm{M}+\mathrm{H})$; HRMS (ESI, m/z) : calcd for $\mathrm{C}_{28} \mathrm{H}_{44} \mathrm{~N}_{2} \mathrm{O}_{4} \mathrm{~F}_{3}:$ : 529.3253, found : 529.3254 .

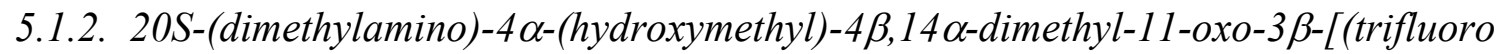
acetyl) amino]-9,19-cyclo-5 $\alpha, 9 \beta$-pregnan-16 $\alpha$-yl acetate (7a).

To $144 \mathrm{mg}(0.27 \mathrm{mmol}, 1$ eq.) of $\mathbf{6 a}$ solubilised in $5 \mathrm{~mL}$ of a mixture DCM/pyridine (3/1) were added at $0{ }^{\circ} \mathrm{C} 27 \mu \mathrm{L}(0.286 \mathrm{mmol}, 1.05$ eq. $)$ of acetic anhydride. The mixture was stirred at $0{ }^{\circ} \mathrm{C}$ for $1 \mathrm{~h}$ then at RT for further $12 \mathrm{~h}$. After 3 co-evaporations with $3 \times 10 \mathrm{~mL}$ of $1,2-$ dichloroethane, the residue was dissolved in DCM, washed with $20 \mathrm{~mL}$ of a saturated solution of sodium hydrogen carbonate then extracted with $3 \times 20 \mathrm{~mL}$ of DCM. Combined organic layers were washed with brine $(20 \mathrm{~mL})$, dried over magnesium sulfate and concentrated under vacuum. The crude product was purified by neutral alumina column chromatography (eluent : $\mathrm{DCM} / \mathrm{MeOH}: 99 / 1$ to $97 / 3$ ) to afford the title compound as an amorphous white solid (112 mg, yield $72 \%)$. IR $\left(\mathrm{cm}^{-1}\right): 3296,2966,2935,2873,2832,1716,1698,1668,1558$, $1456,1251,1208,1185,1156 ;{ }^{1} \mathrm{H} \operatorname{NMR}\left(300 \mathrm{MHz}, \mathrm{CDCl}_{3}\right): \delta(\mathrm{ppm}) 6.90\left(1 \mathrm{H}, \mathrm{d}, \mathrm{J}_{\mathrm{NAH}-\mathrm{H} 3}=\right.$ $\left.9.0 \mathrm{~Hz}, \mathrm{~N}_{\mathrm{A}}-\mathrm{H}\right), 5.11(1 \mathrm{H}, \mathrm{m}, \mathrm{H} 16 \beta), 4.07(1 \mathrm{H}, \mathrm{m}, \mathrm{H} 3 \alpha), 3.40\left(1 \mathrm{H}, \mathrm{d}, \mathrm{J}_{\text {gem }}=12.9 \mathrm{~Hz}, \mathrm{H} 29 \mathrm{~b}\right)$, $3.00\left(1 \mathrm{H}, \mathrm{d}, \mathrm{J}_{\text {gem }}=12.9 \mathrm{~Hz}, \mathrm{H} 29 \mathrm{a}\right), 2.53\left(1 \mathrm{H}, \mathrm{d}, \mathrm{J}_{\text {gem }}=17.2 \mathrm{~Hz}, \mathrm{H} 12 \alpha\right), 2.46(2 \mathrm{H}, \mathrm{m}$, $\mathrm{H} 1 \beta+\mathrm{H} 20), 2.36\left(1 \mathrm{H}, \mathrm{d}, \mathrm{J}_{\mathrm{gem}}=17.2 \mathrm{~Hz}, \mathrm{H} 12 \beta\right), 2.21\left(1 \mathrm{H}, \mathrm{dd}, \mathrm{J}_{17 \alpha-20}=11.1 \mathrm{~Hz}, \mathrm{~J}_{17 \alpha-16 \beta}=\right.$ $5.8 \mathrm{~Hz}, \mathrm{H} 17 \alpha), 2.13\left(6 \mathrm{H}, \mathrm{sl}, \mathrm{N}_{\mathrm{B}} \mathrm{Me}_{2}\right), 2.00-2.09$ (3H, m, H5 $\left.\alpha+\mathrm{H} 8 \beta+\mathrm{H} 15 \beta\right), 1.98$ (3H, s, H32), 1.62-1.79 (3H, m, H2 $\alpha+\mathrm{H} 2 \beta+\mathrm{H} 6 \alpha), 1.56\left(1 \mathrm{H}, \mathrm{d}, \mathrm{J}_{\mathrm{gem}}=3.8 \mathrm{~Hz}, \mathrm{H} 19 \beta\right), 1.51(1 \mathrm{H}, \mathrm{m}, \mathrm{H} 7 \beta)$, $1.42(1 \mathrm{H}, \mathrm{m}, \mathrm{H} 15 \alpha), 1.25-1.36(2 \mathrm{H}, \mathrm{m}, \mathrm{H} 7 \alpha+\mathrm{H} 1 \alpha), 1.14(3 \mathrm{H}, \mathrm{s}, \mathrm{H} 28), 1.05\left(1 \mathrm{H}, \mathrm{d}, \mathrm{J}_{\mathrm{gem}}=\right.$ $3.8 \mathrm{~Hz}, \mathrm{H} 19 \beta), 0.90$ (1H, m, H6ß), $0.83(3 \mathrm{H}, \mathrm{s}, \mathrm{H} 18), 0.80\left(3 \mathrm{H}, \mathrm{d}, \mathrm{J}_{21-20}=6.3 \mathrm{~Hz}, \mathrm{H} 21\right), 0.62$ $(3 \mathrm{H}, \mathrm{s}, \mathrm{H} 30) ;{ }^{13} \mathrm{C}$ NMR $\left(75.4 \mathrm{MHz}, \mathrm{CDCl}_{3}\right): \delta(\mathrm{ppm}) 211.3(\mathrm{C} 11), 170.6(\mathrm{C} 31), 158.4\left(\mathrm{q}, \mathrm{J}_{\mathrm{C} 1}{ }^{\prime}-\mathrm{F}=\right.$

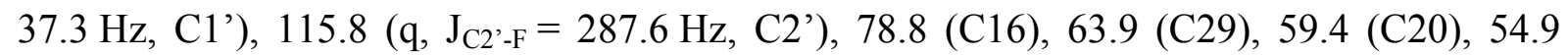
(C17), 52.2 (C3), 51.8 (C12), 47.3 (C14), 44.6 (C4), 44.4 (C13), 42.9 (C15), 41.3 (C8), 41.2 
(C5), $40.1\left(\mathrm{~N}_{\mathrm{B}} \mathrm{Me}_{2}\right), 37.3$ (C10), 33.8 (C9), 30.5 (C19), 27.4 (C1), 27.0 (C2), 24.2 (C7), 21.8 (C32), 19.5 (C28), 18.2 (C6), 17.8 (C18), 11.1 (C30), 9.77 (C21) ; MS (ESI, m/z) : 571.3 $(\mathrm{M}+\mathrm{H}), 529.3$; HRMS (ESI, m/z) : calcd for $\mathrm{C}_{30} \mathrm{H}_{46} \mathrm{~N}_{2} \mathrm{O}_{5} \mathrm{~F}_{3}: 571.3359$, found : 571.3385 .

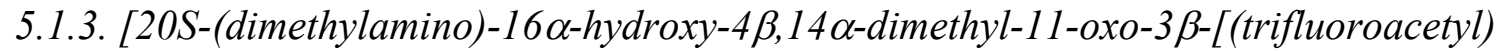

amino]- 9,19-cyclo-5 $\alpha, 9 \beta$-pregnan-4 $\alpha$-yl\}methyl tosylate (8a).

To $110 \mathrm{mg}(0.19 \mathrm{mmol}, 1$ eq. $)$ of $7 \mathbf{a}$ solubilised in $5 \mathrm{~mL}$ of anhydrous $\mathrm{MeOH}$ were added $37 \mathrm{mg}(0.19 \mathrm{mmol}, 1$ eq.) of $p$-toluenesulfonic acid and a small amount of sodium sulfate. The mixture was stirred at RT for $1 \mathrm{~h}$ and filtered through a pad of celite. The solvent was removed and the residue was dried several minutes under vacuum. $1.5 \mathrm{~mL}$ of pyridine and $110 \mathrm{mg}\left(0.19 \mathrm{mmol}, 1\right.$ eq.) of tosyl chloride were added. The mixture was stirred at $90{ }^{\circ} \mathrm{C}$ for $3 \mathrm{~h}$. After 3 co-evaporations with $3 \times 10 \mathrm{~mL}$ of 1,2-dichloroethane, the residue was dissolved in $10 \mathrm{~mL}$ of a mixture $\mathrm{MeOH} /$ water (3/1) and a small amount of potassium carbonate was added. The mixture was stirred at $\mathrm{RT}$ for $3 \mathrm{~h}$ and the $\mathrm{MeOH}$ was removed under vacuum. The mixture was extracted with $3 \times 10 \mathrm{~mL}$ of DCM. Combined organic layers were washed with brine, dried over magnesium sulfate and concentrated under vacuum. The crude product was purified by silica gel column chromatography (eluent : $\mathrm{DCM} / \mathrm{MeOH} / \mathrm{NH}_{4} \mathrm{OH}:$ 98/1/1) to afford the title compound as an amorphous light yellow solid (70 mg, yield $50 \%)$. IR $\left(\mathrm{cm}^{-1}\right)$ : $3321,2924,2869,1719,1667,1551,1454,1367,1159,1189,1208,1175 ;{ }^{1} \mathrm{H}$ NMR (300 $\left.\mathrm{MHz}, \mathrm{CDCl}_{3}\right): \delta(\mathrm{ppm}) 7.77(2 \mathrm{H}, \mathrm{d}, \mathrm{J}=8.3 \mathrm{~Hz}, \mathrm{H} 32+\mathrm{H} 36), 7.34(\mathrm{~d}, 2 \mathrm{H}, \mathrm{J}=8.3 \mathrm{~Hz}, \mathrm{H} 33+\mathrm{H} 35)$, $5.92\left(1 \mathrm{H}, \mathrm{d}, \mathrm{J}_{\mathrm{NAH}-\mathrm{H} 3}=9.8 \mathrm{~Hz}, \mathrm{~N}_{\mathrm{A}}-\mathrm{H}\right), 4.27-4.08(2 \mathrm{H}, \mathrm{m}, \mathrm{H} 3 \alpha+\mathrm{H} 16 \beta), 3.86\left(1 \mathrm{H}, \mathrm{d}, \mathrm{J}_{\mathrm{gem}}=\right.$ $9.9 \mathrm{~Hz}, \mathrm{H} 29 \mathrm{~b}), 3.43\left(1 \mathrm{H}, \mathrm{d}, \mathrm{J}_{\mathrm{gem}}=9.9 \mathrm{~Hz}, \mathrm{H} 29 \mathrm{a}\right), 2.65(1 \mathrm{H}, \mathrm{m}, \mathrm{H} 20), 2.53\left(1 \mathrm{H}, \mathrm{d}, \mathrm{J}_{\mathrm{gem}}=\right.$

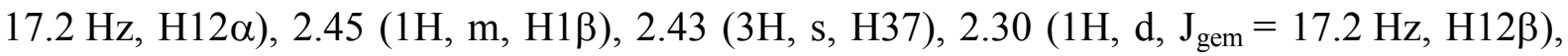
$2.28\left(6 \mathrm{H}, \mathrm{sl}, \mathrm{N}_{\mathrm{B}} \mathrm{Me}_{2}\right), 2.01(4 \mathrm{H}, \mathrm{m}, \mathrm{H} 17 \alpha+\mathrm{H} 5 \alpha+\mathrm{H} 8 \beta+\mathrm{H} 15 \beta), 1.79(1 \mathrm{H}, \mathrm{m}, \mathrm{H} 2 \alpha), 1.56(1 \mathrm{H}$, m, H2 $\beta), 1.54\left(1 \mathrm{H}, \mathrm{d}, \mathrm{J}_{\mathrm{gem}}=3.9 \mathrm{~Hz}, \mathrm{H} 19 \beta\right), 1.51(1 \mathrm{H}, \mathrm{m}, \mathrm{H} 7 \beta), 1.50(2 \mathrm{H}, \mathrm{m}, \mathrm{H} 6 \alpha+\mathrm{H} 15 \alpha)$, 
$1.34(1 \mathrm{H}, \mathrm{m}, \mathrm{H} 7 \alpha), 1.29(1 \mathrm{H}, \mathrm{m}, \mathrm{H} 1 \alpha), 1.19(3 \mathrm{H}, \mathrm{s}, \mathrm{H} 28), 1.01\left(1 \mathrm{H}, \mathrm{d}, \mathrm{J}_{\mathrm{gem}}=3.9 \mathrm{~Hz}, \mathrm{H} 19 \beta\right)$, $0.94(1 \mathrm{H}, \mathrm{m}, \mathrm{H} 6 \beta), 0.89\left(3 \mathrm{H}, \mathrm{d}, \mathrm{J}_{21-20}=6.5 \mathrm{~Hz}, \mathrm{H} 21\right), 0.84(3 \mathrm{H}, \mathrm{s}, \mathrm{H} 18), 0.75(3 \mathrm{H}, \mathrm{s}, \mathrm{H} 30)$;

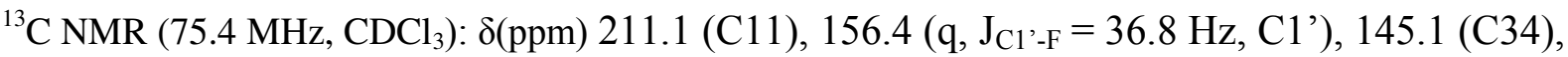
$131.9(\mathrm{C} 31), 129.8(\mathrm{C} 33+\mathrm{C} 35), 128.1(\mathrm{C} 32+\mathrm{C} 36), 115.7\left(\mathrm{q}, \mathrm{J}_{\mathrm{C}^{\prime}-\mathrm{F}}=288.2 \mathrm{~Hz}, \mathrm{C} 2{ }^{\prime}\right), 78.0$ (C16), 70.2 (C29), 62.1 (C20), 55.8 (C17), 51.4 (C12), 51.3 (C3), 47 (C14), 44.4 (C13), 43.0 (C4), 42.9 (C15), 42.3 (C8), 41.8 (C5), 36.6 (C10), 34.1 (C9), 30.4 (C19), 27.6 (C2), 27.0 (C1), 24.3 (C7), 21.6 (C37), 20.9 (C28), 18.5 (C6), 17.9 (C18), 11.1 (C30), 10.0 (C21) ; MS (ESI, m/z) : $683.3(\mathrm{M}+\mathrm{H}) ;$ HRMS (ESI, m/z) : calcd for $\mathrm{C}_{35} \mathrm{H}_{50} \mathrm{~N}_{2} \mathrm{O}_{6} \mathrm{SF}_{3}:$ 683.3342, found : 683.3305 .

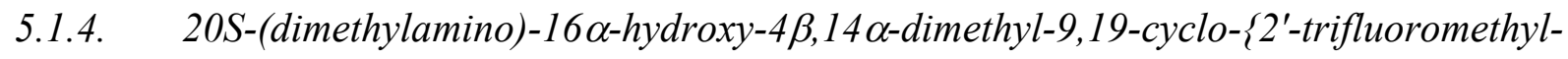
5',6'-dihydro-4' $\alpha H$-[1',3']oxazino[4',5':3,4]\}-5 $\alpha, 9 \beta$-pregnan-11-one (9a).

To $34 \mathrm{mg}$ ( $0.05 \mathrm{mmol}, 1$ eq.) of 8a solubilised in $2 \mathrm{~mL}$ of anhydrous DMF were added $14 \mathrm{mg}$ ( $0.15 \mathrm{mmol}, 3$ eq.) of sodium diformylamide. The mixture was stirred at $120{ }^{\circ} \mathrm{C}$ for $5 \mathrm{~h}$, washed with $20 \mathrm{~mL}$ of a sodium hydrogen carbonate saturated solution and extracted with $5 \times 30 \mathrm{~mL}$ of DCM. Combined organic layers were washed with brine, dried over magnesium sulfate and concentrated under vacuum. The crude product was purified by preparative TLC (eluent : $\left.\mathrm{DCM} / \mathrm{MeOH} / \mathrm{NH}_{4} \mathrm{OH}: 89 / 10 / 1\right)$ to afford the title compound as an amorphous colourless solid (17 mg, yield $68 \%$ ). IR $\left(\mathrm{cm}^{-1}\right): 3669,2924\left(v_{\mathrm{a}} \mathrm{C}-\mathrm{H}\right), 1719,1698,1455,1151$, $1209 ;{ }^{1} \mathrm{H}$ NMR $\left(300 \mathrm{MHz}, \mathrm{CDCl}_{3}\right): \delta(\mathrm{ppm}) 4.26(1 \mathrm{H}, \mathrm{m}, \mathrm{H} 16 \beta), 4.17\left(1 \mathrm{H}, \mathrm{d}, \mathrm{J}_{\mathrm{gem}}=10.3 \mathrm{~Hz}\right.$, H29b), 3.99 (1H, d, J gem $=10.3 \mathrm{~Hz}, \mathrm{H} 29 \mathrm{a}), 3.16(1 \mathrm{H}, \mathrm{m}, \mathrm{H} 3 \alpha), 2.79(1 \mathrm{H}, \mathrm{m}, \mathrm{H} 20), 2.53(1 \mathrm{H}$, $\left.\mathrm{d}, \mathrm{J}_{\mathrm{gem}}=17.1 \mathrm{~Hz}, \mathrm{H} 12 \alpha\right), 2.43(1 \mathrm{H}, \mathrm{m}, \mathrm{H} 1 \beta), 2.40\left(6 \mathrm{H}, \mathrm{sl}, \mathrm{N}_{\mathrm{B}} \mathrm{Me}_{2}\right), 2.34\left(1 \mathrm{H}, \mathrm{d}, \mathrm{J}_{\mathrm{gem}}=\right.$

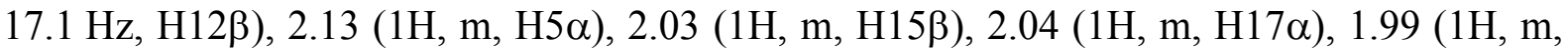
$\mathrm{H} 2 \alpha), 1.68\left(1 \mathrm{H}, \mathrm{d}, \mathrm{J}_{\mathrm{gem}}=4.1 \mathrm{~Hz}, \mathrm{H} 19 \beta\right), 1.58(1 \mathrm{H}, \mathrm{m}, \mathrm{H} 7 \beta), 1.55(1 \mathrm{H}, \mathrm{m}, \mathrm{H} 2 \beta), 1.54(1 \mathrm{H}, \mathrm{m}$,

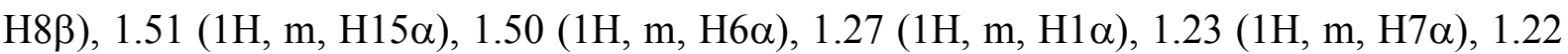


$(3 \mathrm{H}, \mathrm{s}, \mathrm{H} 28), 1.12\left(1 \mathrm{H}, \mathrm{d}, \mathrm{J}_{\mathrm{gem}}=4.1 \mathrm{~Hz}, \mathrm{H} 19 \beta\right), 1.02(1 \mathrm{H}, \mathrm{m}, \mathrm{H} 6 \beta), 0.98\left(3 \mathrm{H}, \mathrm{d}, \mathrm{J}_{21-20}=\right.$ $6.4 \mathrm{~Hz}, \mathrm{H} 21), 0.85$ (3H, s, H18), $0.87(3 \mathrm{H}, \mathrm{s}, \mathrm{H} 30) ;{ }^{13} \mathrm{C} \mathrm{NMR}\left(75.4 \mathrm{MHz}, \mathrm{CDCl}_{3}\right): \delta(\mathrm{ppm}) 211.1$ (C11), 147.0 (C1'), 78.2 (C16), 77.4 (C29), 63.0 (C20), 59.9 (C3), 55.9 (C17), 51.4 (C12), 47.2 (C14), 44.9 (C13), 46.1 (C8), 43.2 (C15), 40.6 (C5), 36.7 (C10), 35.1 (C4), 33.9 (C9), 29.8 (C19), 28.0 (C1), 27.7 (C2), 23.7 (C7), 20.3 (C28), 18.2 (C6), 17.9 (C18), 10.9 (C30), $10.4(\mathrm{C} 21)$. MS (ESI, m/z) : $511.3(\mathrm{M}+\mathrm{H})$; HRMS (ESI, m/z) : calcd for $\mathrm{C}_{28} \mathrm{H}_{42} \mathrm{~N}_{2} \mathrm{O}_{3} \mathrm{~F}_{3}$ : 511.3148 , found : 511.3129 .

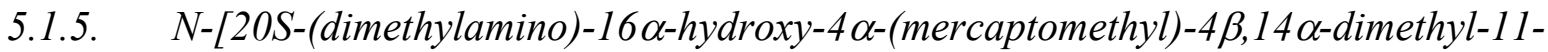
oxo-9,19-cyclo-5 $\alpha, 9 \beta$-pregnan-3 $\beta$-yl]-isobutyramide (29).

To $40 \mathrm{mg}$ ( $0.057 \mathrm{mmol}$, 1 eq.) of $\mathbf{2 8}$ dissolved in $0.5 \mathrm{~mL}$ of DMPU were added $98 \mathrm{mg}$ ( $0.86 \mathrm{mmol}, 15$ eq.) of sodium thioacetate. The mixture was stirred at $100{ }^{\circ} \mathrm{C}$ for $5 \mathrm{~h}$ then diluted with $10 \mathrm{~mL}$ of a saturated sodium hydrogen carbonate solution and extracted with $20 \mathrm{~mL}$ of $\mathrm{Et}_{2} \mathrm{O}$. The organic layer was dried with brine, over magnesium sulfate and concentrated under vacuum. The residue was dissolved in $20 \mathrm{~mL}$ of a degassed mixture $\mathrm{MeOH} /$ water (7/3) and a small amount of potassium hydroxide was added. The mixture was stirred at RT for $3 \mathrm{~h}$. The $\mathrm{MeOH}$ was removed under reduced pressure and the mixture was extracted with $3 \times 20 \mathrm{~mL}$ of DCM. Combined organic layers were washed with brine, dried over magnesium sulfate and concentrated under vacuum. The crude product was purified by silica gel column chromatography (gradient elution : $\mathrm{DCM} / \mathrm{MeOH} / \mathrm{NH}_{4} \mathrm{OH}$ : from $99 / 0 / 1$ to 90/10/1) to afford the title compound as an amorphous white solid (20 mg, yield: $67 \%$ ). IR $\left(\mathrm{cm}^{-1}\right): 3322,2964,2937,2869,2833,1655,1534,1460,1225,1159,1094 ;{ }^{1} \mathrm{H}$ NMR (300 $\left.\mathrm{MHz}, \mathrm{CDCl}_{3}\right): \delta(\mathrm{ppm}) 5.23\left(1 \mathrm{H}, \mathrm{d}, \mathrm{J}_{\mathrm{NAH}-3}=9.9 \mathrm{~Hz}, \mathrm{~N}_{\mathrm{A}}-\mathrm{H}\right), 4.15(1 \mathrm{H}, \mathrm{m}, \mathrm{H} 3 \alpha), 4.12(1 \mathrm{H}, \mathrm{m}$, H16ß), $2.64\left(1 \mathrm{H}, \mathrm{dq}, \mathrm{J}_{20-17}=10.8 \mathrm{~Hz}, \mathrm{~J}_{20-21}=6.5 \mathrm{~Hz}, \mathrm{H} 20\right), 2.53\left(1 \mathrm{H}, \mathrm{d}, \mathrm{J}_{\mathrm{gem}}=17.1 \mathrm{~Hz}\right.$, H12 $\alpha), 2.49(1 \mathrm{H}, \mathrm{m}, \mathrm{H} 29 \mathrm{~b}), 2.41(1 \mathrm{H}, \mathrm{m}, \mathrm{H} 1 \beta), 2.34\left(1 \mathrm{H}, \mathrm{m}, \mathrm{H} 2^{\prime}\right), 2.29\left(1 \mathrm{H}, \mathrm{d}, \mathrm{J}_{\mathrm{gem}}=\right.$ 
17.1 Hz, H12ß), 2.26 (6H, bs, $\left.\mathrm{N}_{\mathrm{B}} \mathrm{Me}\right), 2.22$ (1H, m, H5), 2.19 (1H, m, H29a), 2.02 (2H, m, $\mathrm{H} 8 \beta+\mathrm{H} 15 \beta), 1.99(1 \mathrm{H}, \mathrm{m}, \mathrm{H} 17 \alpha), 1.72(1 \mathrm{H}, \mathrm{m}, \mathrm{H} 2 \alpha), 1.56\left(1 \mathrm{H}, \mathrm{d}, \mathrm{J}_{\mathrm{gem}}=3.4 \mathrm{~Hz}, \mathrm{H} 19 \alpha\right), 1.55$ $(1 \mathrm{H}, \mathrm{m}, \mathrm{H} 7 \beta), 1.51(1 \mathrm{H}, \mathrm{m}, \mathrm{H} 15 \alpha), 1.49(1 \mathrm{H}, \mathrm{m}, \mathrm{H} 2 \beta), 1.38(1 \mathrm{H}, \mathrm{m}, \mathrm{H} 7 \alpha), 1.33(1 \mathrm{H}, \mathrm{m}$, H6 $\alpha), 1.31(1 \mathrm{H}, \mathrm{m}, \mathrm{H} 1 \alpha), 1.23$ (3H, s, H28), 1.17+1.14 (6H, 2d, J = 7.2 Hz, H3'+H4'), 1.03 $\left(1 \mathrm{H}, \mathrm{d}, \mathrm{J}_{\mathrm{gem}}=3.4 \mathrm{~Hz}, \mathrm{H} 19 \beta\right), 0.92(1 \mathrm{H}, \mathrm{m}, \mathrm{H} 6 \beta), 0.89\left(3 \mathrm{H}, \mathrm{d}, \mathrm{J}_{21-20}=6.5 \mathrm{~Hz}, \mathrm{H} 21\right), 0.85(3 \mathrm{H}$, s, H18), $0.78(3 \mathrm{H}, \mathrm{s}, \mathrm{H} 30) ;{ }^{13} \mathrm{C}$ NMR (75.4 MHz, $\left.\mathrm{CDCl}_{3}\right): \delta(\mathrm{ppm}) 211.4(\mathrm{C} 11), 176.7$ (C1'), 78.1 (C16), 62.0 (C20), 55.7 (C17), 51.4 (C12), 49.7 (C3), 47.0 (C14), 44.4 (C13), 43.1 (C4), 42.9 (C15), 42.0 (C5), 41.9 (C8), 37.6 (C10), 35.8 (C2'), 34.2 (C9), 31.2 (C29), 30.9 (C19), 28.5 (C2), 27.4 (C1), 24.3 (C7), 20.8 (C28), 20.1+19.4 (C3'+C4'), 18.4 (C6), 17.8 (C18), $15.5(\mathrm{C} 30), 9.9(\mathrm{C} 21)$; MS (ESI, m/z) : $519.3(\mathrm{M}+\mathrm{H})$; HRMS (ESI, m/z) : calcd for $\mathrm{C}_{30} \mathrm{H}_{51} \mathrm{~N}_{2} \mathrm{O}_{3} \mathrm{~S}: 519.3620$, found : 519.3611 .

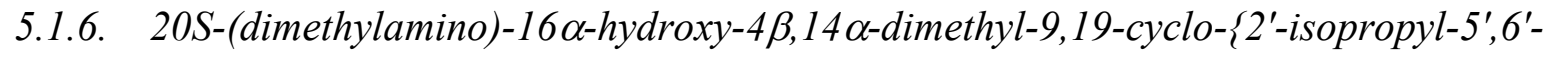

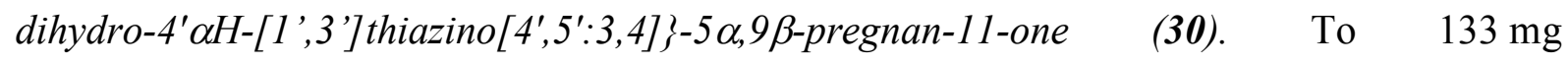
(0.256 mmol, 1 eq.) of 29 dissolved in $5 \mathrm{~mL}$ of 1,2-dichloroethane were added $640 \mu \mathrm{L}$ ( $1.28 \mathrm{mmol}, 5$ eq.) of trimethylaluminium ( $2 \mathrm{M}$ in toluene). The mixture was refluxed for $3 \mathrm{~h}$ then cooled to RT. The mixture was quenched with $3 \mathrm{~mL}$ of sodium hydroxide $(1 \mathrm{~N})$, diluted $10 \mathrm{~mL}$ of a saturated potassium hydrogen carbonate solution and extracted with $3 \times 10 \mathrm{~mL}$ of DCM. Combined organic layers were washed with brine, dried over magnesium sulfate and concentrated under vacuum. The crude product was purified by silica gel column chromatography (gradient elution : $\mathrm{DCM} / \mathrm{MeOH} / \mathrm{NH}_{4} \mathrm{OH}$ : from $99 / 0 / 1$ to $90 / 10 / 1$ ) to afford the title compound as an amorphous white solid (76 mg, yield: $59 \%$ ). IR ( $\left.\mathrm{cm}^{-1}\right): 3330,2962$, 2933, 2866, 1661, 1633, 1462, 1260, 1124, 1157, $1088 ;{ }^{1} \mathrm{H}$ NMR (300 MHz, $\left.\mathrm{CDCl}_{3}\right): \delta(\mathrm{ppm})$ $4.10(1 \mathrm{H}, \mathrm{m}, \mathrm{H} 16 \beta), 2.84(1 \mathrm{H}, \mathrm{m}, \mathrm{H} 3 \alpha), 2.83\left(1 \mathrm{H}, \mathrm{d}, \mathrm{J}_{\mathrm{gem}}=11.6 \mathrm{~Hz}, \mathrm{H} 29 \mathrm{~b}\right), 2.73(1 \mathrm{H}, \mathrm{d}$, $\left.\mathrm{J}_{\mathrm{gem}}=11.6 \mathrm{~Hz}, \mathrm{H} 29 \mathrm{a}\right), 2.63\left(1 \mathrm{H}, \mathrm{dq}, \mathrm{J}_{20-17 \alpha}=9.9 \mathrm{~Hz}, \mathrm{~J}_{20-21}=6.6 \mathrm{~Hz}, \mathrm{H} 20\right), 2.58(1 \mathrm{H}, \mathrm{m}, \mathrm{H} 2$ ') 
$2.51\left(1 \mathrm{H}, \mathrm{d}, \mathrm{J}_{\mathrm{gem}}=17.0 \mathrm{~Hz}, \mathrm{H} 12 \alpha\right), 2.45(1 \mathrm{H}, \mathrm{dt}, \mathrm{J}=13.4 \mathrm{~Hz}, \mathrm{~J}=3.5 \mathrm{~Hz}, \mathrm{H} 1 \beta), 2.32(1 \mathrm{H}, \mathrm{d}$, $\left.\mathrm{J}_{\mathrm{gem}}=17.0 \mathrm{~Hz}, \mathrm{H} 12 \beta\right), 2.25\left(6 \mathrm{H}, \mathrm{bs}, \mathrm{N}_{\mathrm{B}} \mathrm{Me}_{2}\right), 2.10(1 \mathrm{H}, \mathrm{m}, \mathrm{H} 8 \beta), 2.05$ (1H, m, H2 2$), 2.01$ (1H, m, H15ß), $1.98(1 \mathrm{H}, \mathrm{m}, \mathrm{H} 17 \alpha), 1.71(1 \mathrm{H}, \mathrm{m}, \mathrm{H} 2 \beta), 1.64\left(1 \mathrm{H}, \mathrm{d}, \mathrm{J}_{\mathrm{gem}}=3.9 \mathrm{~Hz}, \mathrm{H} 19 \alpha\right)$, $1.58(1 \mathrm{H}, \mathrm{m}, \mathrm{H} 7 \beta), 1.53$ (1H, m, H6 $\alpha), 1.49$ (1H, m, H15 $\alpha), 1.42(1 \mathrm{H}, \mathrm{m}, \mathrm{H} 5 \alpha), 1.34$ (1H, m, H7 $\alpha), 1.31(1 \mathrm{H}, \mathrm{m}, \mathrm{H} 1 \alpha), 1.21(3 \mathrm{H}, \mathrm{s}, \mathrm{H} 28), 1.17$ and $1.18\left(6 \mathrm{H}, 2 \mathrm{~d}, \mathrm{~J}=6.9 \mathrm{~Hz}, \mathrm{H} 3{ }^{\prime}+\mathrm{H} 4{ }^{\prime}\right)$, $1.11\left(1 \mathrm{H}, \mathrm{d}, \mathrm{J}_{\mathrm{gem}}=3.9 \mathrm{~Hz}, \mathrm{H} 19 \beta\right), 1.01(1 \mathrm{H}, \mathrm{m}, \mathrm{H} 6 \beta), 0.88\left(3 \mathrm{H}, \mathrm{d}, \mathrm{J}_{21-20}=6.6 \mathrm{~Hz}, \mathrm{H} 21\right), 0.85$ $(3 \mathrm{H}, \mathrm{s}, \mathrm{H} 18), 0.76(3 \mathrm{H}, \mathrm{s}, \mathrm{H} 30) ;{ }^{13} \mathrm{C} \mathrm{NMR}\left(75.4 \mathrm{MHz}, \mathrm{CDCl}_{3}\right): \delta(\mathrm{ppm}) 210.8(\mathrm{C} 11), 165.7$ (C1'), 78.3 (C16), 62.7 (C3), 62.0 (C20), 55.7 (C17), 51.4 (C12), 49.8 (C5), 47.1 (C14), 44.5 (C13), 42.8 (C15), 41.4 (C8), 40.2 (C2’), 38.7 (C29), 38.4 (C10), 34.5 (C9), 33.4 (C4), 30.6 (C2), 30.2 (C19), 28.0 (C1), 24.4 (C7), 20.8 and 21.0 (C3'+C4'), 20.7 (C28), 18.0 (C6), 17.9 (C18), 11.3 (C30), 9.9 (C21); MS (ESI, m/z) : $501.3(\mathrm{M}+\mathrm{H})$; HRMS (ESI, m/z) : calcd for $\mathrm{C}_{30} \mathrm{H}_{49} \mathrm{~N}_{2} \mathrm{O}_{2} \mathrm{~S}: 501.3515$, found : 501.3537.

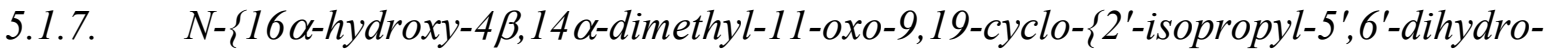
$4^{\prime} \alpha H-\left[1^{\prime}, 3\right.$ '] thiazino $\left.\left[4^{\prime}, 5^{\prime}: 3,4\right]\right\}-5 \alpha, 9 \beta$-pregnan-20S-yl\}-N-methylacetamide (35a).

To $12 \mathrm{mg}$ ( $0.0246 \mathrm{mmol}, 1$ eq.) of $\mathbf{3 4}$ dissolved in $1 \mathrm{~mL}$ of absolute EtOH were added $3 \mu \mathrm{L}$ ( $0.032 \mathrm{mmol}, 1.3$ eq.) of acetic anhydride. The mixture was stirred at RT for $3 \mathrm{~h}$ and concentrated under vacuum. $5 \mathrm{~mL}$ of a saturated sodium hydrogen carbonate solution were added and the mixture was extracted with $3 \times 5 \mathrm{~mL}$ of DCM. Combined organic layers were washed with brine, dried over magnesium sulfate and concentrated under vacuum. The crude product was purified by silica gel column chromatography (gradient elution : DCM/MeOH/ $\mathrm{NH}_{4} \mathrm{OH}$ : from $99 / 0 / 1$ to $97 / 2 / 1$ ) to afford the title compound as an amorphous white solid (12 mg, yield: $92 \%)$. IR $\left(\mathrm{cm}^{-1}\right): 3306,2962,2930,2868,1662,1612,1454,1258$, 1114, 1082, $1016 ;{ }^{1} \mathrm{H}$ NMR $\left(300 \mathrm{MHz}, \mathrm{CDCl}_{3}\right): \delta(\mathrm{ppm}) 4.99\left(1 \mathrm{H}, \mathrm{dq}, \mathrm{J}_{20-17}=11.3 \mathrm{~Hz}, \mathrm{~J}_{20-21}=\right.$ $6.8 \mathrm{~Hz}, \mathrm{H} 20), 4.20(1 \mathrm{H}, \mathrm{m}, \mathrm{H} 16 \beta), 2.89\left(3 \mathrm{H}, \mathrm{s}, \mathrm{N}_{\mathrm{B}} \mathrm{Me}\right), 2.88(1 \mathrm{H}, \mathrm{m}, \mathrm{H} 3 \alpha), 2.84(1 \mathrm{H}, \mathrm{d}$, 
$\left.\mathrm{J}_{\mathrm{gem}}=11.5 \mathrm{~Hz}, \mathrm{H} 29 \mathrm{~b}\right), 2.74\left(1 \mathrm{H}, \mathrm{d}, \mathrm{J}_{\text {gem }}=11.5 \mathrm{~Hz}, \mathrm{H} 29 \mathrm{a}\right), 2.58(1 \mathrm{H}, \mathrm{m}, \mathrm{H} 2$ ') $2.57(1 \mathrm{H}, \mathrm{d}$, $\left.\mathrm{J}_{\mathrm{gem}}=17.0 \mathrm{~Hz}, \mathrm{H} 12 \alpha\right), 2.48\left(1 \mathrm{H}, \mathrm{dt}, \mathrm{J}_{\mathrm{gem}}=13.6 \mathrm{~Hz}, \mathrm{~J}_{1 \beta-2}=3.2 \mathrm{~Hz}, \mathrm{H} 1 \beta\right), 2.37\left(1 \mathrm{H}, \mathrm{d}, \mathrm{J}_{\mathrm{gem}}=\right.$ $17.0 \mathrm{~Hz}, \mathrm{H} 12 \beta), 2.11$ (1H, m, H17 $\alpha), 2.08$ (3H, s, H32), 2.07 (1H, m, H8 $\beta), 2.02$ (1H, m, H15ß), $1.73(1 \mathrm{H}, \mathrm{m}, \mathrm{H} 2 \alpha), 1.65\left(1 \mathrm{H}, \mathrm{d}, \mathrm{J}_{\mathrm{gem}}=4.1 \mathrm{~Hz}, \mathrm{H} 19 \alpha\right), 1.62(1 \mathrm{H}, \mathrm{m}, \mathrm{H} 2 \beta), 1.54(2 \mathrm{H}$,

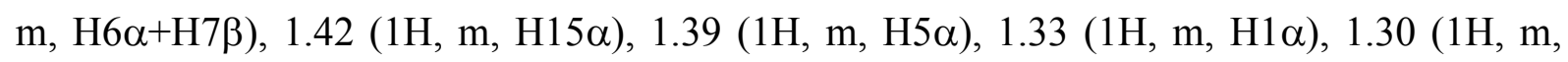
$\mathrm{H} 7 \alpha), 1.20(3 \mathrm{H}, \mathrm{s}, \mathrm{H} 28), 1.20+1.18\left(6 \mathrm{H}, 2 \mathrm{~d}, \mathrm{~J}=7.0 \mathrm{~Hz}, \mathrm{H} 3^{\prime}+\mathrm{H}^{\prime}{ }^{\prime}\right), 1.13\left(1 \mathrm{H}, \mathrm{d}, \mathrm{J}_{\mathrm{gem}}=4.1 \mathrm{~Hz}\right.$, H19ß), $1.09\left(3 \mathrm{H}, \mathrm{d}, \mathrm{J}_{21-20}=6.8 \mathrm{~Hz}, \mathrm{H} 21\right), 1.03(1 \mathrm{H}, \mathrm{m}, \mathrm{H} 6 \beta), 0.96(3 \mathrm{H}, \mathrm{s}, \mathrm{H} 18), 0.77(3 \mathrm{H}, \mathrm{s}$, $\mathrm{H} 30) ;{ }^{13} \mathrm{C} \mathrm{NMR}\left(75.4 \mathrm{MHz}, \mathrm{CDCl}_{3}\right): \delta(\mathrm{ppm}) 210.4$ (C11), 173.7 (C31), $171.0(\mathrm{C} 1$ ') $75.6(\mathrm{C} 16)$, 62.6 (C3), 57.1 (C17), 51.6 (C12), 49.8 (C5), 48.2 (C20), 47.4 (C14), 46.1 (C15), 45.6 (C13), 41.4 (C8), 40.1 (C2'), 38.7 (C29), 38.4 (C10), 34.0 (C9), 33.5 (C4), 30.5 (C2+C19), 30.4 ( $\left.\mathrm{N}_{\mathrm{B}} \mathrm{Me}\right), 27.9$ (C1), 24.5 (C7), 22.5 (C32), 21.1+20.8 (C3'+C4'), 19.9 (C28), 18.1 (C21), 18.0 (C18+C6), $11.3(\mathrm{C} 30)$; MS (ESI, m/z) : $529.3(\mathrm{M}+\mathrm{H})$; HRMS (ESI, m/z) : calcd for $\mathrm{C}_{31} \mathrm{H}_{49} \mathrm{~N}_{2} \mathrm{O}_{3} \mathrm{~S}:$ : 529.3464, found : 529.3461 .

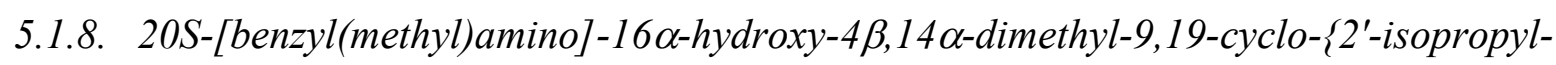
5',6'-dihydro-4' $\alpha H-\left[1^{\prime}, 3^{\prime}\right]$ thiazino $\left.\left[4^{\prime}, 5^{\prime}: 3,4\right]\right\}-5 \alpha, 9 \beta$-pregnan-11-one $\quad$ (36a). To $13 \mathrm{mg}$ ( $0.0267 \mathrm{mmol}, 1$ eq.) of $\mathbf{3 4}$ dissolved in $1 \mathrm{~mL}$ of acetonitrile were added $4.1 \mu \mathrm{L}(0.035 \mathrm{mmol}$, 1.3 eq. $)$ of benzyl bromide and $11 \mathrm{mg}(0.08 \mathrm{mmol}, 3$ eq. $)$ of potassium carbonate. The mixture was stirred at RT for $16 \mathrm{~h}$, diluted with $5 \mathrm{~mL}$ of a saturated sodium hydrogen carbonate solution then extracted with $3 \times 5 \mathrm{~mL}$ of DCM. Combined organic layers were washed with brine, dried over magnesium sulfate and concentrated under vacuum. The crude product was purified by silica gel column chromatography (gradient elution: DCM/MeOH/ $\mathrm{NH}_{4} \mathrm{OH}$ : from $99 / 0 / 1$ to $96 / 3 / 1$ ) to afford the title compound as an amorphous white solid (14 mg, yield: $93 \%)$. IR $\left(\mathrm{cm}^{-1}\right): 3324,2962,2929,2867,1666,1633,1452,1259,1223,1147,1079,1017$; ${ }^{1} \mathrm{H}$ NMR (500 MHz, CD $\left.{ }_{3} \mathrm{CN}, 333 \mathrm{~K}\right): \delta(\mathrm{ppm}) 7.30$ (5H, m, H33+H34+H35+H36+H37), 3.99 (1H, 
m, H16ß), $3.72\left(1 \mathrm{H}, \mathrm{d}, \mathrm{J}_{\text {gem }}=12.8 \mathrm{~Hz}, \mathrm{H} 31\right), 3.59\left(1 \mathrm{H}, \mathrm{d}, \mathrm{J}_{\mathrm{gem}}=12.8 \mathrm{~Hz}, \mathrm{H} 31\right), 2.86(2 \mathrm{H}, \mathrm{m}$, H20+H29b), $2.84(1 \mathrm{H}, \mathrm{m}, \mathrm{H} 3 \alpha), 2.75\left(1 \mathrm{H}, \mathrm{d}, \mathrm{J}_{\text {gem }}=11.6 \mathrm{~Hz}, \mathrm{H} 29 \mathrm{a}\right), 2.53\left(1 \mathrm{H}, \mathrm{d}, \mathrm{J}_{\text {gem }}=\right.$ $17.1 \mathrm{~Hz}, \mathrm{H} 12 \alpha), 2.48\left(1 \mathrm{H}, \mathrm{m}, \mathrm{H} 2\right.$ '), $2.35\left(1 \mathrm{H}, \mathrm{dt}, \mathrm{J}_{\mathrm{gem}}=13.6 \mathrm{~Hz}, \mathrm{~J}_{1 \beta-2}=3.2 \mathrm{~Hz}, \mathrm{H} 1 \beta\right), 2.23$ $\left(1 \mathrm{H}, \mathrm{d}, \mathrm{J}_{\mathrm{gem}}=17.1 \mathrm{~Hz}, \mathrm{H} 12 \beta\right), 2.21\left(3 \mathrm{H}, \mathrm{s}, \mathrm{N}_{\mathrm{B}} \mathrm{Me}\right), 2.12(1 \mathrm{H}, \mathrm{m}, \mathrm{H} 17 \alpha), 2.08(1 \mathrm{H}, \mathrm{m}, \mathrm{H} 8 \beta)$, $1.97(1 \mathrm{H}, \mathrm{m}, \mathrm{H} 15 \beta), 1.95(1 \mathrm{H}, \mathrm{m}, \mathrm{H} 2 \alpha), 1.63(1 \mathrm{H}, \mathrm{m}, \mathrm{H} 2 \beta), 1.53(1 \mathrm{H}, \mathrm{m}, \mathrm{H} 7 \beta), 1.51(1 \mathrm{H}, \mathrm{m}$, H6 $\alpha), 1.50\left(1 \mathrm{H}, \mathrm{d}, \mathrm{J}_{\mathrm{gem}}=4.0 \mathrm{~Hz}, \mathrm{H} 19 \alpha\right), 1.47(1 \mathrm{H}, \mathrm{m}, \mathrm{H} 5 \alpha), 1.39(1 \mathrm{H}, \mathrm{m}, \mathrm{H} 15 \alpha), 1.33(1 \mathrm{H}$, m, H7 $), 1.31(1 \mathrm{H}, \mathrm{m}, \mathrm{H} 1 \alpha), 1.18(3 \mathrm{H}, \mathrm{s}, \mathrm{H} 28), 1.13(1 \mathrm{H}, \mathrm{m}, \mathrm{H} 19 \beta), 1.12(6 \mathrm{H}, 2 \mathrm{~d}, \mathrm{~J}=$ 6.7 Hz, H3'+H4'), 1.05 (1H, m, H6ß), 0.98 (3H, d, J $21-20=6.4 \mathrm{~Hz}, \mathrm{H} 21), 0.75$ (3H, s, H18), $0.73(3 \mathrm{H}, \mathrm{s}, \mathrm{H} 30) ;{ }^{13} \mathrm{C}$ NMR (75.4 MHz, $\left.\mathrm{CD}_{3} \mathrm{CN}\right): \delta(\mathrm{ppm}) 210.8(\mathrm{C} 11), 165.9\left(\mathrm{C}^{\prime}\right.$ '), 138.7 (C32), 129.1 (C33+C37), 128.5 (C34+C36), 127.3 (C35), 77.6 (C16), 62.7 (C3), 60.3 (C20), 59.4 (C31), 55.8 (C17), 51.4 (C12), 49.7 (C5), 47.2 (C14), 44.7 (C13), 42.8 (C15), 41.3 (C8), 40.2 (C2'), 38.7 (C29), 38.4 (C10), 35.3 (N $\left.\mathrm{N}_{\mathrm{B}} \mathrm{Me}\right), 34.4$ (C9), 33.4 (C4), 30.5 (C2), 30.3 (C19), 28.0 (C1), 24.4 (C7), 21.0+20.8 (C3’+C4'), 20.6 (C28), 18.0 (C6), 17.7 (C18), 11.3 (C30), 10.7 (C21); MS (ESI, m/z) : $577.4(\mathrm{M}+\mathrm{H}), 487.3(\mathrm{M}-\mathrm{Bn}+\mathrm{H})$; HRMS (ESI, m/z) : calcd for $\mathrm{C}_{36} \mathrm{H}_{53} \mathrm{~N}_{2} \mathrm{O}_{2} \mathrm{~S}: 577.3828$, found : 577.3835 .

\subsection{Biochemical Methods}

\subsubsection{In vitro AChE Inhibition Assay}

Enzymes : EeAChE from electrophorus electricus (reference C 2888) and human recombinant $h \mathrm{AChE}$ (reference C 1682) were purchased from Sigma.

Inhibition of AChE activity was determined by the spectroscopic method of Ellman et al. [14], using acetylthiocholine iodide as substrate, in 96-well microtiter plates. All solutions were brought to room temperature prior to use. Aliquots of $200 \mu \mathrm{L}$ of a solution containing $640 \mu \mathrm{L} 10 \mathrm{mM}$ DTNB in $0.1 \mathrm{M}$ sodium phosphate, $\mathrm{pH} 8.0,19.2 \mathrm{~mL}$ of the same buffer, and $13 \mu \mathrm{L}$ of a solution of $\mathrm{AChE}(100 \mathrm{U} / \mathrm{mL})$ in water, were added to each well, followed by $2 \mu \mathrm{L}$ 
of a DMSO solution of the inhibitor. The reaction was initiated by adding $20 \mu \mathrm{L}$ of acetylthiocholine iodide $(7.5 \mathrm{mM})$ to each well, and followed by monitoring the appearance of the thiolate dianion produced by reduction of DTNB at $412 \mathrm{~nm}$ for $120 \mathrm{~s}$ at $25^{\circ} \mathrm{C}$ in a Molecular Devices Spectra Max 384 Plus plate reader. Each inhibitor was evaluated at several concentrations in the range of $10^{-9}-2 \times 10^{-5} \mathrm{M}$. Percentage inhibition was calculated relative to a control sample (DMSO), and $\mathrm{IC}_{50}$ values displayed represent the mean \pm standard deviation for triplicate assays.

\subsubsection{In vitro BuChE Inhibition Assay}

Enzyme : $h$ BuChE from human serum (reference C 9971) was purchased from Sigma. Inhibition of BuChE activity was determined by the spectroscopic method of Ellman et al. [14], using butyrylthiocholine iodide as substrate, in 96-well microtiter plates. All solutions were brought to room temperature prior to use. Aliquots of $200 \mu \mathrm{L}$ of a solution containing $640 \mu \mathrm{L} 10 \mathrm{mM}$ DTNB in $0.1 \mathrm{M}$ sodium phosphate, $\mathrm{pH} 8.0,19.2 \mathrm{~mL}$ of the same buffer, and $13 \mu \mathrm{L}$ of a solution of $\mathrm{BuChE}(100 \mathrm{U} / \mathrm{mL})$ in water, were added to each well, followed by 2 $\mu \mathrm{L}$ of a DMSO solution of the inhibitor. The reaction was initiated by adding $20 \mu \mathrm{L}$ of butyrylthiocholine iodide $(7.5 \mathrm{mM})$ to each well, and followed by monitoring the appearance of the thiolate dianion produced by reduction of DTNB at $412 \mathrm{~nm}$ for $120 \mathrm{~s}$ at $25^{\circ} \mathrm{C}$ in a Molecular Devices Spectra Max 384 Plus plate reader. Each inhibitor was evaluated at several concentrations in the range of $10^{-9}-2 \times 10^{-5} \mathrm{M}$. Percentage inhibition was calculated relative to a control sample, and $\mathrm{IC}_{50}$ values displayed represent the mean \pm standard deviation for triplicate assays.

\subsubsection{Inhibition of AChE-Induced A $\beta_{40}$ Aggregation Assay}

Thioflavine T (T3516), 1,1,1,3,3,3,-hexafluoro-2-propanol (HFIP) and human recombinant 
hAChE (reference C 1682) were purchased from Sigma Chemicals. $\beta$-amyloid - $_{100}(\mathrm{~A} \beta 40)$ trifluoroacetate salt was purchased form Bachem AG (Bubendorf, Switzerland). Aliquots of 2 $\mu \mathrm{L}$ of $\mathrm{A} \beta(1-40)$, lyophilized from $2 \mathrm{mg} / \mathrm{mL}$ HFIP and dissolved in DMSO at a final concentration of $230 \mu \mathrm{M}$, were incubated for $24 \mathrm{~h}$ at room temperature in $0.215 \mathrm{M}$ sodium phosphate buffer (pH 8.0). For coincubation experiments, aliquots of $h \mathrm{AChE}(2.30 \mu \mathrm{M}$, ratio 100:1) and $h \mathrm{AChE}$ in the presence of the tested compound $(100 \mu \mathrm{M})$ were added. Blanks containing $\mathrm{A} \beta, h \mathrm{AChE}, \mathrm{A} \beta$ plus the tested compound, and $h \mathrm{AChE}$ plus the tested compound in $0.215 \mathrm{M}$ sodium phosphate buffer ( $\mathrm{pH} 8.0$ ) were prepared. The final volume of each vial was $20 \mu \mathrm{L}$. To quantify amyloid fibril formation, the thioflavin $\mathrm{T}$ fluorescence method was used $^{9}$ and monitored with a spectrofluorometer (Hitachi F-2500). Thioflavin $\mathrm{T}$ binds to amyloid fibrils, giving rise to an intense specific emission band at $490 \mathrm{~nm}$ in its fluorescent emission spectrum. Therefore, after incubation, the samples were diluted to a final volume of $2 \mathrm{~mL}$ with $50 \mathrm{mM}$ glycine- $\mathrm{NaOH}$ buffer $(\mathrm{pH} 8.5$ ) containing $1.5 \mu \mathrm{M}$ thioflavin $\mathrm{T}$. A $300 \mathrm{~s}$ time scan of fluorescence intensity was carried out $(\lambda \operatorname{exc}=446 \mathrm{~nm}, \lambda \mathrm{em}=490 \mathrm{~nm})$, and values at the plateau were averaged after subtraction of the background fluorescence of the $1.5 \mu \mathrm{M}$ thioflavin $\mathrm{T}$ solution. The percent inhibition of the AChE-induced aggregation due to the presence of increasing concentrations of the inhibitor was calculated by the following expression: 100-[(IF $\left.\left./ \mathrm{IF}_{0}\right) \times 100\right]$ where $\mathrm{IF}_{\mathrm{i}}$ and $\mathrm{IF}_{\mathrm{o}}$ were the fluorescence intensities obtained for $\mathrm{A} \beta$ plus $\mathrm{AChE}$ in the presence and in the absence of inhibitor, respectively, after subtracting the fluorescence of respective blanks. Each assay was run in triplicate.

\subsection{Computer-Aided Molecular Modeling}

Molecular docking was carried out using GOLD 4.0 [22] with standard parameters. Crystal structure of EeAChE (pdb code 1EEA) has been chosen in order to facilitate the comparison 
with the inhibition assay results, the binding site being defined as a $20 \AA$ radius sphere around the $\mathrm{OH}$ group of Ser200. Although this protein has been crystallized in the apo form, the residues that form the anionic site are positioned in the open form of the gorge, thus making this structure appropriate for docking compounds presumed to interact with both the catalytic and peripheral sites of EeAChE. The 3D structures of ligands were constructed with CORINA 3.44 (Molecular Networks GmbH).

Molecular dynamics simulations were carried out with GROMACS version 4.0.2 [23] using the OPLS-AA [24] force field. Each system was energy-minimized until convergence using a steepest descents algorithm. Molecular dynamics with position restraints for $200 \mathrm{ps}$ was then performed followed by the production run of $10 \mathrm{~ns}$. During the position restraints and production runs, the Parinello-Rahman method [25] was used for pressure coupling, and the temperature was coupled using the Nosé-Hoover method [26] at $300 \mathrm{~K}$. Electrostatics were calculated with the particle mesh Ewald method [27]. The P-LINCS algorithm [28] was used to constrain bond lengths, and a time step of 2 fs was used throughout. Ligand topologies for the OPLS-AA force field were obtained using an in-house developed script. All calculations were performed using the HPC facilities at the ICSN.

Images were generated with Chimera [29] and raytraced with the POV-ray module.

Acknowledgment We thank the CNRS and ICSN for financial support. J. Rouleau was supported by fellowship from the Institut de Chimie des Substances Naturelles (ICSNCNRS). Professor J.L. Lallemand is gratefully acknowledged for his interest in our work. We also thank Dr. Jordi Molgo for fruitful discussions. 


\section{Appendix. Supplementary material}

Detailed experimental procedures, compound characterization data for $\mathbf{6 b}-\mathbf{c}, \mathbf{7 b}-\mathbf{c}, \mathbf{8 b}-\mathbf{c}, \mathbf{9 b}-\mathbf{c}$, 12a-b, 13a-b, 14, 15, 16, 18, 19, 20, 21, 22, 23, 24, 25, 26, 27, 31, 32, 33, 34, 35b-c, 36b-k. Computer-Aided Molecular Modeling. RMSD, RMSF and protein-ligand contact surface plots from MD simulations. Equilibrium conformations for compounds $\mathbf{1}$ and $\mathbf{3 6 e}$ in the AChE binding site can be found in the online version at .....

\section{References}

[1] J. Hardy, D. J. Selkoe, Science 297 (2002) 353-356.

[2] M. Tolnay, A. Probst, Neuropathol. Appl. Neurobiol. 25 (1999) 171-187.

[3] R. T. Bartus, R. L. Dean, $3^{\text {rd }}$; B. Beer, A. S. Lippa, Science 217 (1982) 408-414.

[4] S. B. Dunnett, H. C. Fibiger, Prog. Brain Res. 98, (1999) 413-420.

[5] R. T. Bartus, Exp. Neurol. 163 (2000) 495-529.

[6] D. Munoz-Torrero, P. Camps, Curr. Med. Chem. 13 (2006) 399-422.

[7] D. Munoz-Torrero, Curr. Med. Chem. 15 (2008) 2433-2455.

[8] T. Sauvaître, M. Barlier, D. Herlem, N. Gresh, A. Chiaroni, D. Guenard, C. Guillou, J. Med. Chem. 50 (2007) 5311-323.

[9] M. Bartolini, C. Bertucci, V. Cavrini, V. Andriasino, Biochem. Pharmacol. 65 (2003) 407-416.

[10] W.K. Hagmann, J. Med. Chem. 51 (2008) 4259-4369.

[11] H. K. Nair, K. Lee, D.M. Quinn, J. Am. Chem. Soc. 115 (1993) 9939-9941.

[12] J. T. Shaw, W. L. Corbett, D. L. Layman, G. D. Cuny, J. Kerschner, J. Heterocyclic Chem. 25 (1988) 1837-1840. 
[13] C. Guillou, T. Sauvaitre, D. Guénard, J.Y. Lallemand, D. Herlem, J. Molgo, F. KhuongHuu, WO 2006082126.

[14] G. L. Ellman, K. D. Courtney, V. Andres, R. M. Featherstone, Biochem. Pharmacol. 7 (1961) 88-95.

[15] E. Giacobini, Butyrylcholinesterase its Functions and Inhibitors. Martin Dunitz : London 2003.

[16] D.E. Kuhl, R.A. Koeppe, S.E. Snyder, S. Minoshima, K.A. Frey, M.R. Kilbourn, Ann. Neurol. 59, (2006) 13-20.

[17] M. L. Bolognesi, V. Andrisano, M. Bartolini, R. Banzi, C. Melchiorre, J. Med. Chem. 48 (2005) 24-27.

[18] P. Munoz-Ruiz, L. Rubio, E. Garcia-Palomero, I. Dorronsoro, M. del Monte-Millan, R. Valenzuela, P. Usan, C. de Austria, M. Bartolini, V. Andrisano, A. Bidon-Chanal, M. Orozco, F. J. Luque, M. Medina, A. Martınez, J. Med. Chem. 48 (2005)7223-7233.

[19] A. Cavalli, M. L. Bolognesi, S. Capsoni, V. Andrisano, M. Bartolini, E. Margotti, A. Cattaneo, M. Recanatini, C. Melchiorre, Angew. Chem., Int. Ed. 46 (2007) 3689-3692.

[20] Bolognesi, M. L.; Banzi, R.; Bartolini, M.; Cavalli, A.; Tarozzi, A.; Andrisano, V.; Minarini, A.; Rosini, M.; Tumiatti, V.; Bergamini, C.; Fato, R.; Lenaz, G.; Hrelia, P.; Cattaneo, A.; Recanatini, M.; Melchiorre, C. Novel Class of Quinone-Bearing Polyamines as Multi-Target-Directed Ligands to Combat Alzheimer's Disease. J. Med. Chem. 2007, 50, $4882-4897$.

[21] Q. Xie, H. Wang, Z. Xia, M. Lu, W. Zhang, X. Wang, W. Fu, Y. Tang, W. Sheng, W. Li, W. Zhou, X. Zhu, Z. Qiu, H. Chen, J. Med. Chem. 51 (2008) 2027-2036. 
[22] M. L. Verdonk, J. C. Cole, M. J. Hartshorn, C. W. Murray, R. D. Taylor, Proteins 52 (2003) 609-623.

[23] B. Hess, C. Kutzner, D. van der Spoel, E. Lindahl, J. Chem. Theory Comput. 4 (2008) $435-447$.

[24] W. L. Jorgensen, D. S. Maxwell, J. Tirado-Rives, J. Am. Chem. Soc. 118 (1996) 11225 11236.

[25] M. Parinello, A. Rahman, J. Appl. Phys. 52 (1981) 7182-7190.

[26] S. Nosé, Mol. Phys. 52 (1984) 255-268.

[27] U. Essman, L. Perela, M. L. Berkowitz, T. Darden, H. Lee, L. G. Pedersen, J. Chem. Phys. 103 (1995) 8577-8592.

[28] B. Hess, J. Chem. Theory Comput. 4 (2008) 116-122.

[29] E. F. Pettersen, T. D. Goddard, C. C. Huang, G. S. Couch, D. M. Greenblatt, E. C. Meng, T. E. Ferrin, J. Comput. Chem. 25 (2004) 1605-12. 


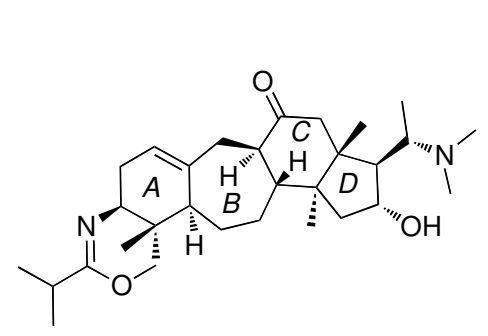

1: oxazine

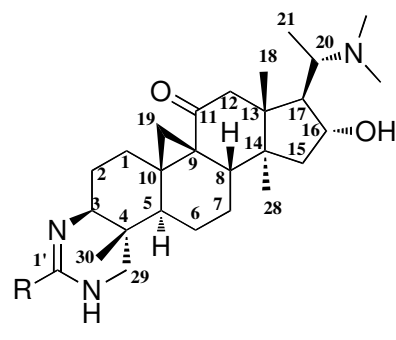

2a: $\mathrm{R}_{1}=\mathrm{iPr}$

2b: $\mathrm{R}_{1}=(S)-\left(\mathrm{CH}_{3}\right) \mathrm{CH}\left(\mathrm{C}_{2} \mathrm{H}_{5}\right)$

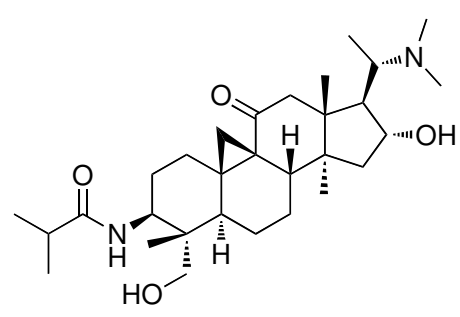

3 : N-3-isobutyrylcycloxobuxidine-F

Figure 1. 


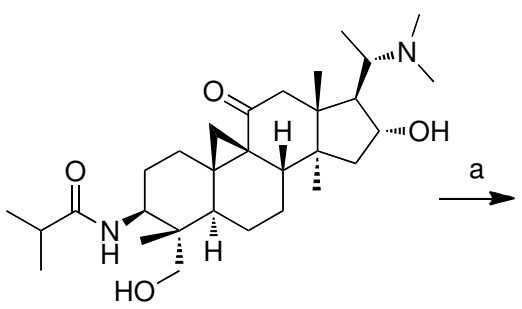

3: N-3-isobutyrylcycloxobuxidine-F

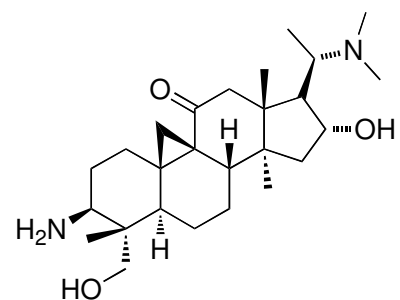

$4(94 \%)$

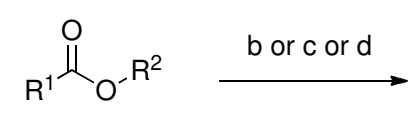

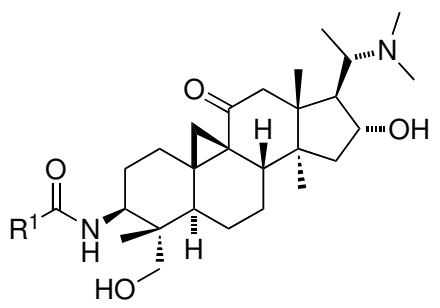

5a $\quad \mathrm{R}^{1}=\mathrm{CF}_{3} ; \mathrm{R}^{2}=\mathrm{COCF}_{3}$

5b $\mathrm{R}^{1}=\left(\mathrm{CH}_{3}\right)_{2} \mathrm{CF} ; \mathrm{R}^{2}=\mathrm{H}$

5c $\mathrm{R}^{1}=\left(\mathrm{CF}_{3}\right)_{2} \mathrm{CF} ; \mathrm{R}^{2}=\mathrm{OCH}_{3}$

6a $\mathrm{R}^{1}=\mathrm{CF}_{3}(84 \%)$

6b $\mathrm{R}^{1}=\left(\mathrm{CH}_{3}\right)_{2} \mathrm{CF}(86 \%)$

6c $\mathrm{R}^{1}=\left(\mathrm{CF}_{3}\right)_{2} \mathrm{CF}(73 \%)$

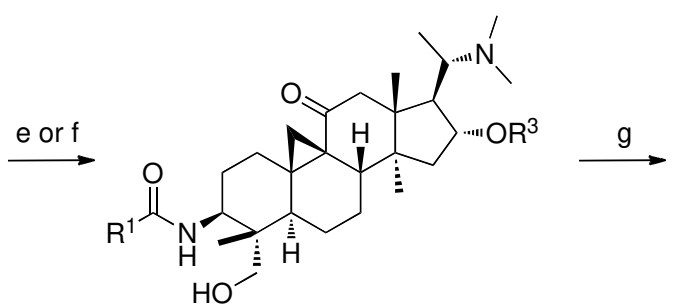

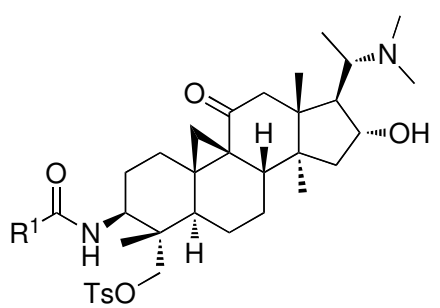

7a $\mathrm{R}^{1}=\mathrm{CF}_{3} ; \mathrm{R}^{3}=\mathrm{COCH}_{3}(72 \%)$

7b $\mathrm{R}^{1}=\left(\mathrm{CH}_{3}\right)_{2} \mathrm{CF} ; \mathrm{R}^{3}=\mathrm{COCH}_{3}(86 \%)$

7c $\mathrm{R}^{1}=\left(\mathrm{CF}_{3}\right)_{2} \mathrm{CF} ; \mathrm{R}^{3}=\mathrm{COPh}(95 \%)$

8a $\quad \mathrm{R}^{1}=\mathrm{CF}_{3}(50 \%)$

8b $\mathrm{R}^{1}=\left(\mathrm{CH}_{3}\right)_{2} \mathrm{CF}(66 \%)$

8c $R^{1}=\left(\mathrm{CF}_{3}\right)_{2} \mathrm{CF}(31 \%)$

$\stackrel{\mathrm{h}}{\longrightarrow}$

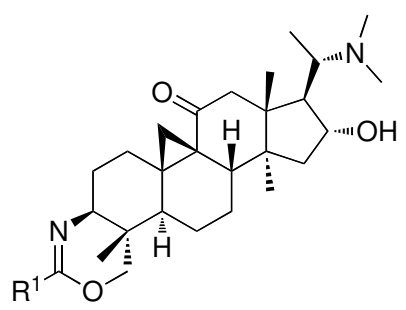

9a $\mathrm{R}^{1}=\mathrm{CF}_{3}(68 \%)$

9b $\mathrm{R}^{1}=\left(\mathrm{CH}_{3}\right) \mathrm{CF}(32 \%)$

9c $\mathrm{R}^{1}=\left(\mathrm{CF}_{3}\right)_{2} \mathrm{CF}(14 \%)$

aReagents and conditions : (a) $\mathrm{H}_{2} \mathrm{SO}_{4}, \mathrm{MeOH}$; (b) for $5 \mathbf{a}$ : pyridine, $\mathrm{CH}_{2} \mathrm{Cl}_{2}$; ( c) for $5 \mathbf{b}$ : $(\mathrm{CICO})_{2}$ DMF then pyridine, $\mathrm{CH}_{2} \mathrm{Cl}_{2}$; (d) for $5 \mathbf{c}: \mathrm{MeOH} ;(\mathrm{e})$ for $\mathbf{6 a}$ and $\mathbf{6 b}: \mathrm{Ac}_{2} \mathrm{O}$, pyridine, $\mathrm{CH}_{2} \mathrm{Cl}_{2}$; (f) for $7 \mathbf{c}$ : $\mathrm{Bz}_{2} \mathrm{O}$, pyridine, $\mathrm{CH}_{2} \mathrm{Cl}_{2}$; (g) i ) APTS, $\mathrm{Na}_{2} \mathrm{SO}_{4}, \mathrm{MeOH}$, ii) $\mathrm{TsCl}$, pyridine, iii) $\mathrm{K}_{2} \mathrm{CO}_{3}, \mathrm{MeOH}, \mathrm{H}_{2} \mathrm{O}$; (h) $\mathrm{NaN}(\mathrm{CHO})_{2}$, DMF

Scheme 1. 


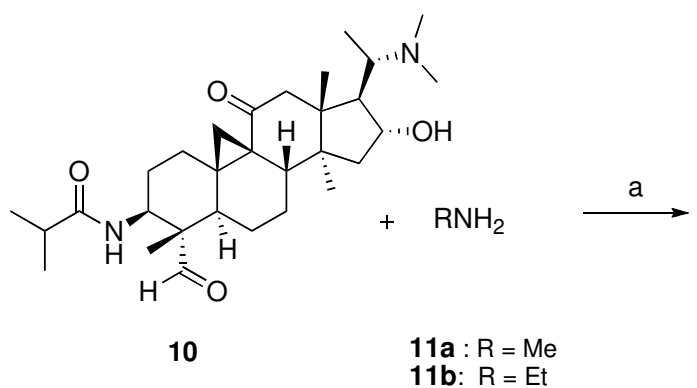

11b: $R=E t$

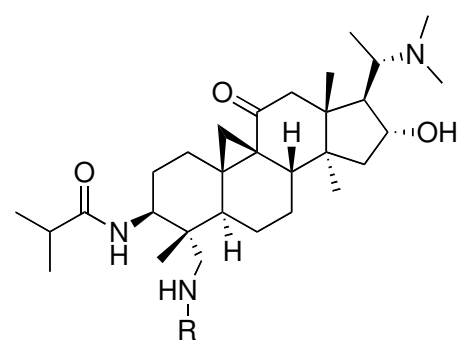

12a $: R=\operatorname{Me}(90 \%)$

12b : R $=\mathrm{Et}(85 \%)$
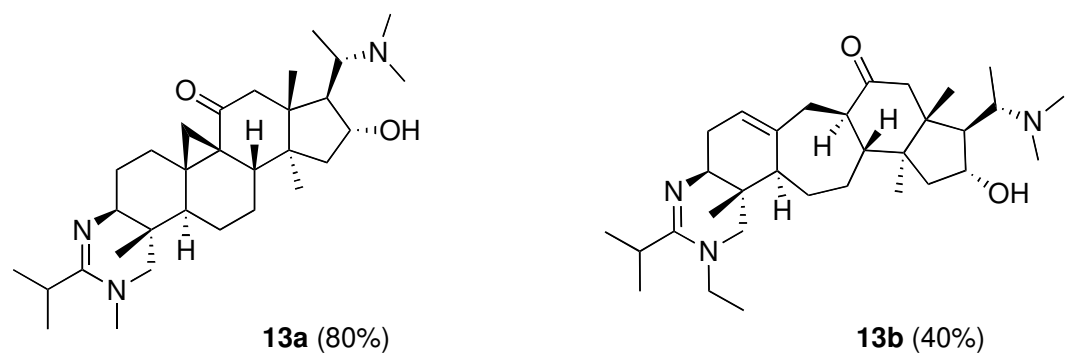

aReagents and conditions : (a) i) $\mathrm{RNH}_{2}, \mathrm{Na}_{2} \mathrm{SO}_{4}, \mathrm{EtOH}$, ii) $\mathrm{NaBH}_{3} \mathrm{CN}, \mathrm{AcOH}, \mathrm{EtOH}$; (b) $\mathrm{NEt}_{3}$, $n$-BuOH, microwaves, $200^{\circ} \mathrm{C}, 2 \mathrm{~h}$; (c) $\mathrm{NEt}_{3}, n-\mathrm{BuOH}$, microwaves, $250^{\circ} \mathrm{C}, 5 \mathrm{~h}$.

Scheme 2. 


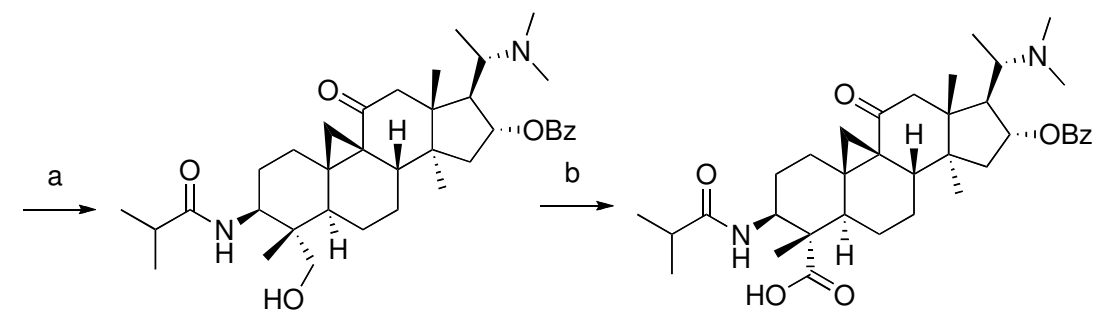

14

15

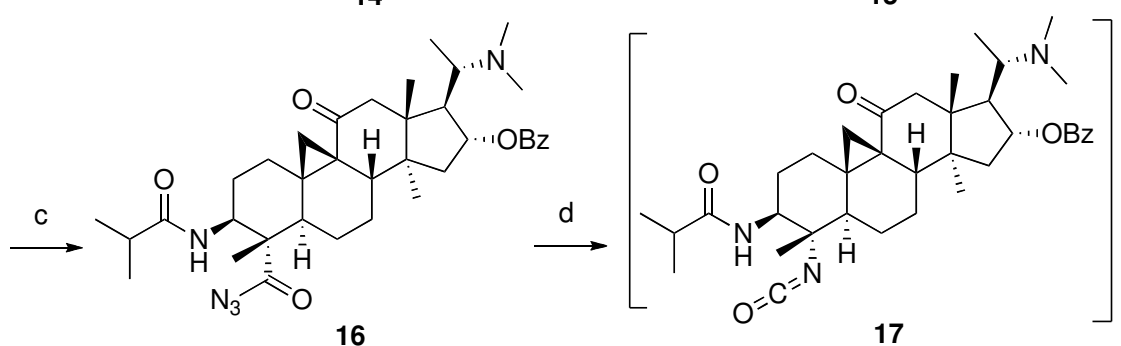

16

17

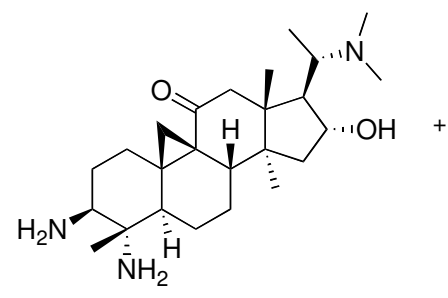

$18 \quad 18 / 19: 90 / 10$

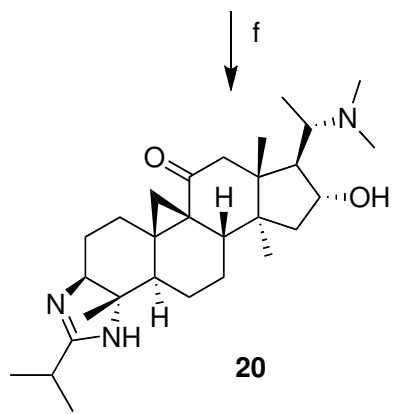

'Reagents and conditions : (a) $\mathrm{Bz}_{2} \mathrm{O}$, pyridine, $\mathrm{CH}_{2} \mathrm{Cl}_{2}$; (b) Jones' reagent, acetone (73\%); (c) i) $(\mathrm{ClCO})_{2}, \mathrm{NEt}_{3}$, DMF, ii) $\mathrm{NaN}_{3}, \mathrm{H}_{2} \mathrm{O}(74 \%)$; (d) microwaves (100\%); (e) i) $\mathrm{LiOH}, \mathrm{THF}, \mathrm{H}_{2} \mathrm{O}$, ii) $\mathrm{MeOH}$, iii) $1 \mathrm{~N} \mathrm{HCl}$ reflux $(84 \%)$; (f) $\operatorname{irC}(\mathrm{OMe})_{3}, \mathrm{Yb}(\mathrm{OTf})_{3}$, Dioxane $(85 \%)$.

Scheme 3. 


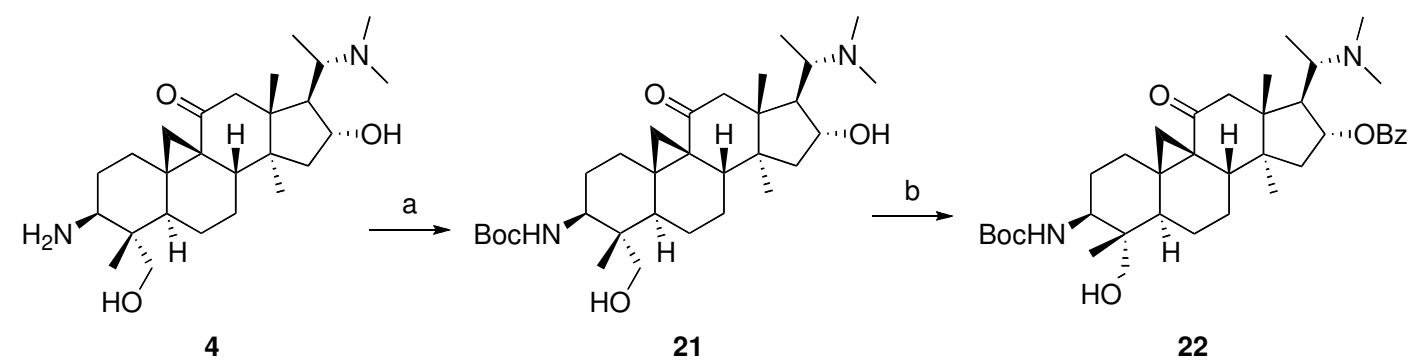

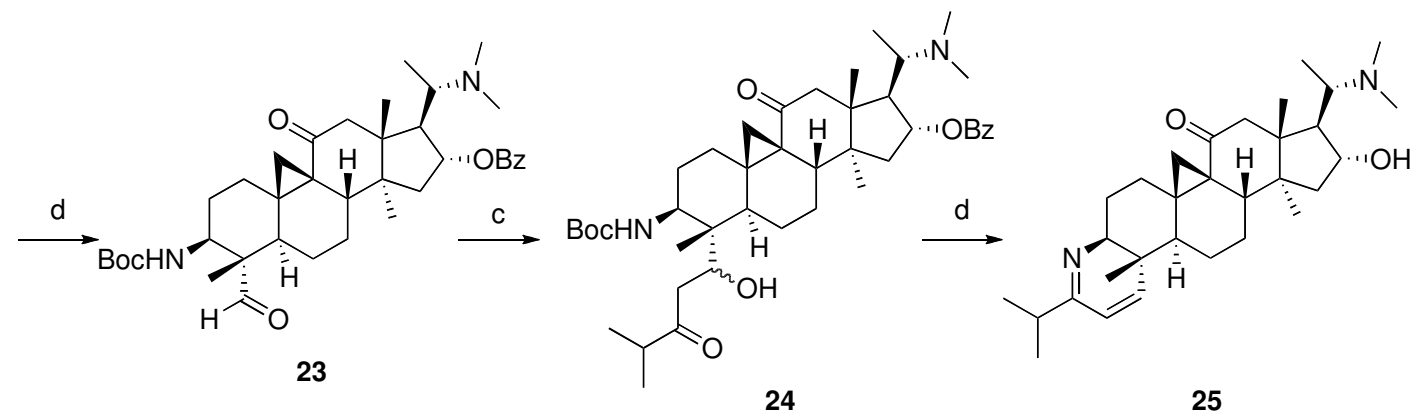

aReagents and conditions : (a) $\mathrm{Boc}_{2} \mathrm{O}, \mathrm{MeOH}$ (100\%); (b) $\mathrm{Bz}_{2} \mathrm{O}, \mathrm{DMAP}, \mathrm{CH}_{2} \mathrm{Cl}_{2}, \mathrm{NEt}_{3}(97 \%)$; (b) Dess-Martin periodinane, $\mathrm{CH}_{2} \mathrm{Cl}_{2}$ (88\%); (c) $\operatorname{iPrCOCH}{ }_{3}$, LDA, THF (79\%); (d) i) TFA, $\mathrm{CH}_{2} \mathrm{Cl}_{2}$, ii) $\mathrm{K}_{2} \mathrm{CO}_{3}, \mathrm{MeOH}, \mathrm{H}_{2} \mathrm{O}(63 \%)$.

Scheme 4.

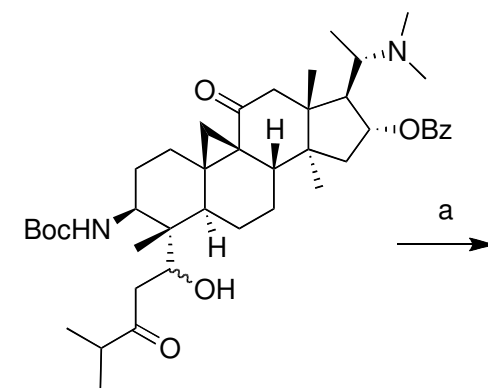

24

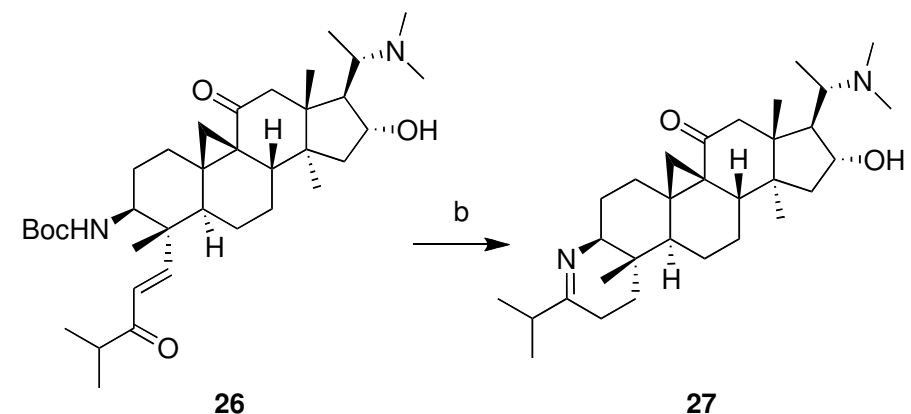

27

aReagents and conditions : (a) $\mathrm{K}_{2} \mathrm{CO}_{3}, \mathrm{MeOH}, \mathrm{H}_{2} \mathrm{O}$ (86\%). (b) i) $\mathrm{Pd}(\mathrm{OH})_{2}, \mathrm{H}_{2}, \mathrm{MeOH}$, ii) TFA, $\mathrm{CH}_{2} \mathrm{Cl}_{2}(68 \%$ ).

Scheme 5. 


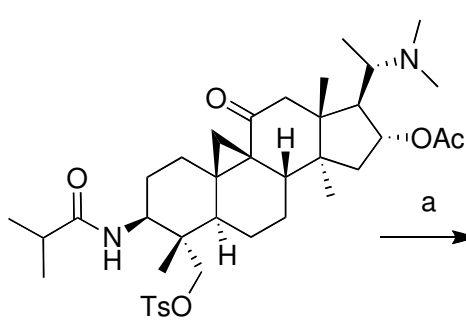

28

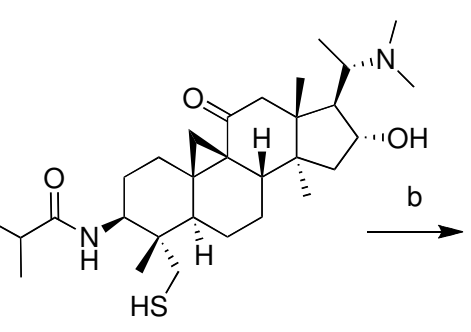

29

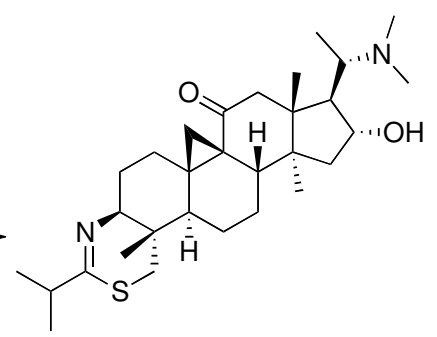

30

aReagents and conditions : (a) i) KSAc, DMPU, ii) $\mathrm{KOH}, \mathrm{MeOH}, \mathrm{H}_{2} \mathrm{O}(67 \%)$. (b) $\mathrm{AlMe}_{3},\left(\mathrm{ClCH}_{2}\right)_{2}(59 \%)$

Scheme 6. 

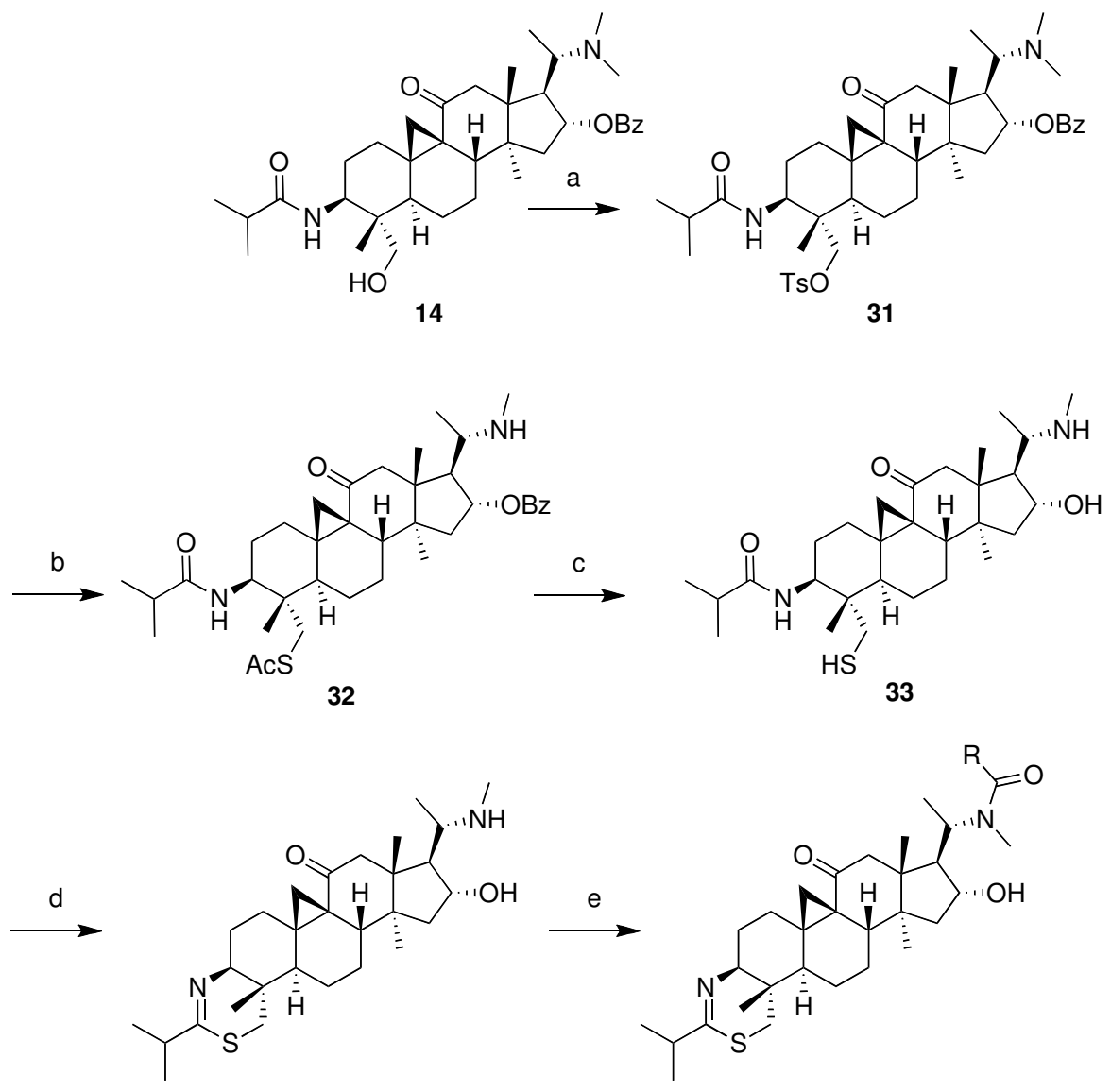

34

$\begin{array}{ll}35 a & R=M e(92 \%) \\ 35 b & R=P h(83 \%)\end{array}$

35c $\mathrm{R}=\mathrm{O} t-\mathrm{Bu}(83 \%)$

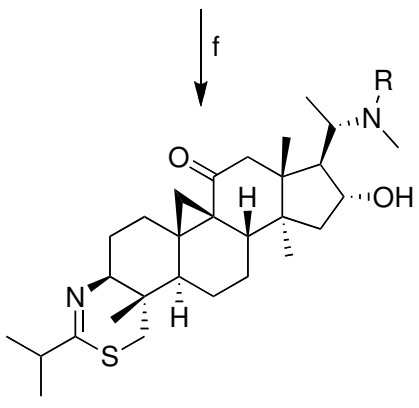

36a $\mathrm{R}=\mathrm{Bn}(93 \%)$

36b $\mathrm{R}=\mathrm{CH}_{2} \mathrm{Bn}$ (84\%)

36c $\mathrm{R}=\left(\mathrm{CH}_{2}\right)_{2} \mathrm{Bn}(72 \%)$

36d $\mathrm{R}=0-\mathrm{OMeBn}(83 \%)$

36e $\mathrm{R}=m-\mathrm{OMeBn}(80 \%)$

$36 f \mathrm{R}=p$-OMeBn $(70 \%)$

36g R $=o-\mathrm{CF}_{3} \mathrm{Bn}(55 \%)$

36h $\mathrm{R}=m-\mathrm{CF}_{3} \mathrm{Bn}(62 \%)$

$36 \mathrm{i} \mathrm{R}=\mathrm{p}-\mathrm{CF}_{3} \mathrm{Bn}(75 \%)$

36j $\mathrm{R}=\left(\mathrm{CH}_{2}\right)_{2} \mathrm{Pht}(72 \%)$

36k $\mathrm{R}=\left(\mathrm{CH}_{2}\right)_{3} \mathrm{Pht}(75 \%)$

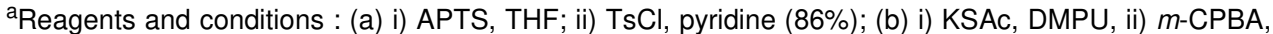
$\mathrm{CH}_{2} \mathrm{Cl}_{2}$, iii) $\mathrm{FeSO}_{4} .7 \mathrm{H}_{2} \mathrm{O}, \mathrm{FeCl}_{3} .6 \mathrm{H}_{2} \mathrm{O}, \mathrm{MeOH}(81 \%)$; (c) $\mathrm{KOH}, \mathrm{MeOH}, \mathrm{H}_{2} \mathrm{O}(55 \%)$; (d) $\mathrm{AlMe}_{3},\left(\mathrm{ClCH}_{2}\right)_{2}$ (70\%); (e) (RCO) $)_{2} \mathrm{O}$, EtOH or THF; (f) $\mathrm{RX}, \mathrm{K}_{2} \mathrm{CO}_{3}, \mathrm{CH}_{3} \mathrm{CN}$ or DMF.

\section{Scheme 7.}




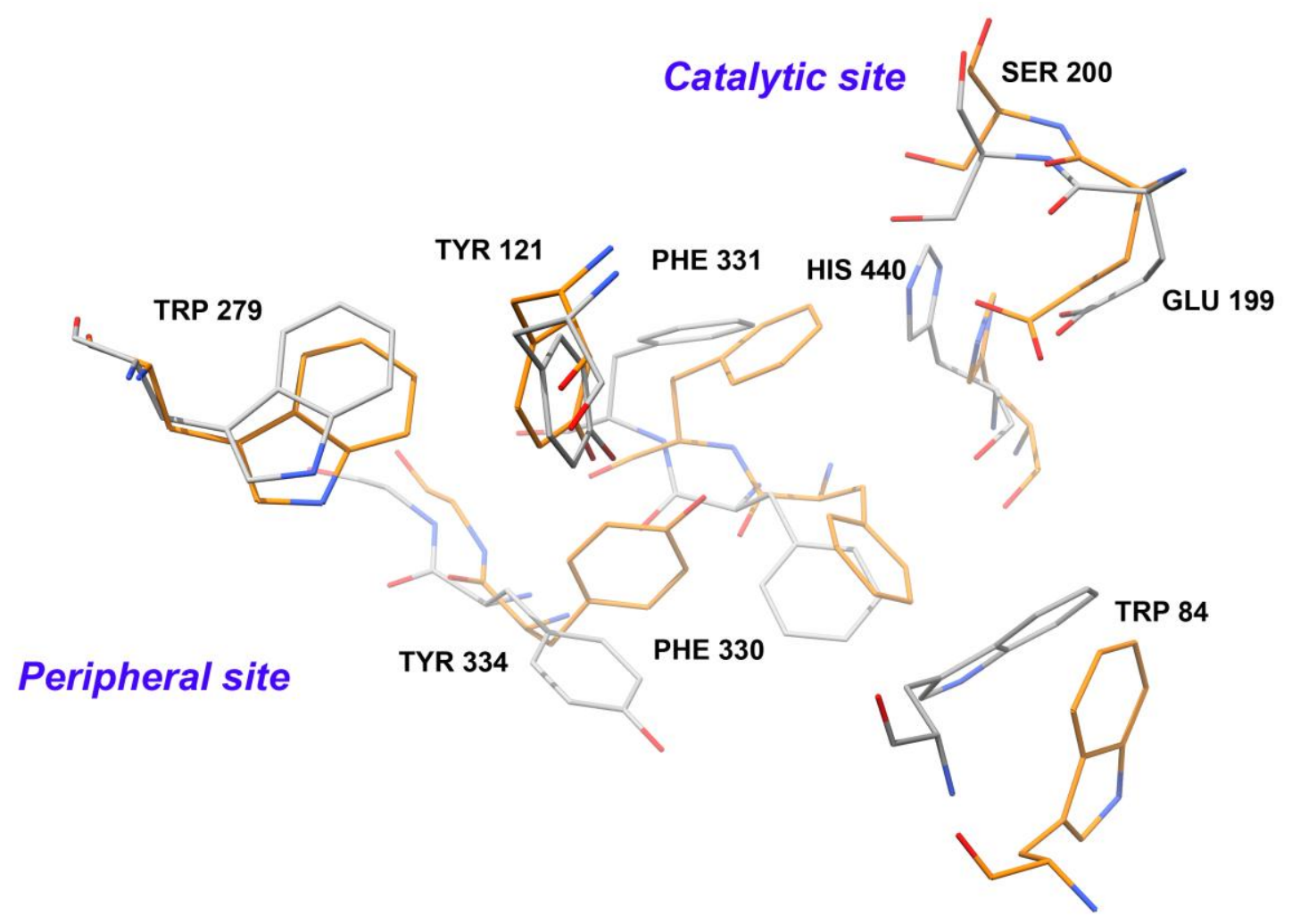

Figure 2 


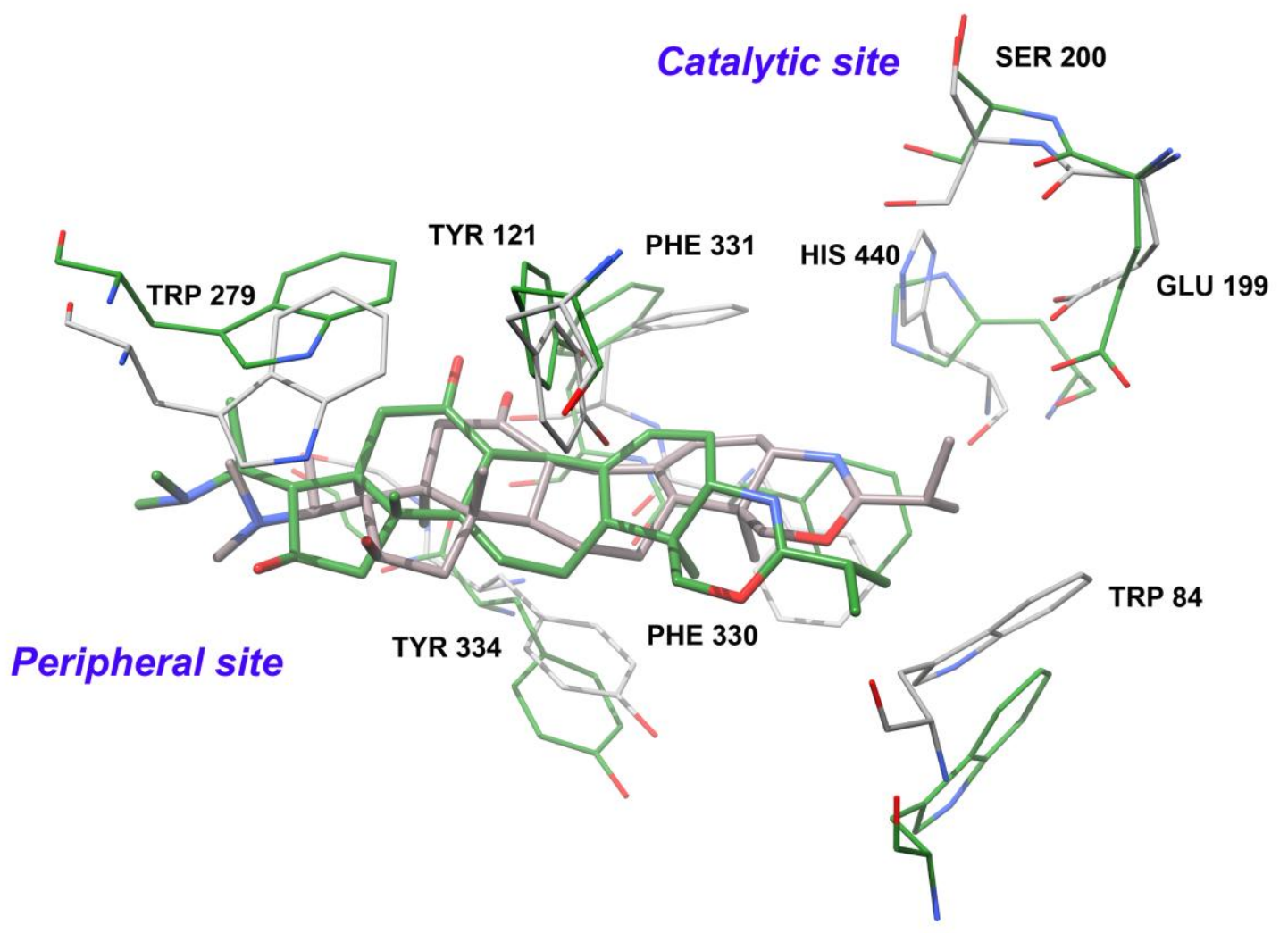

Figure 3 


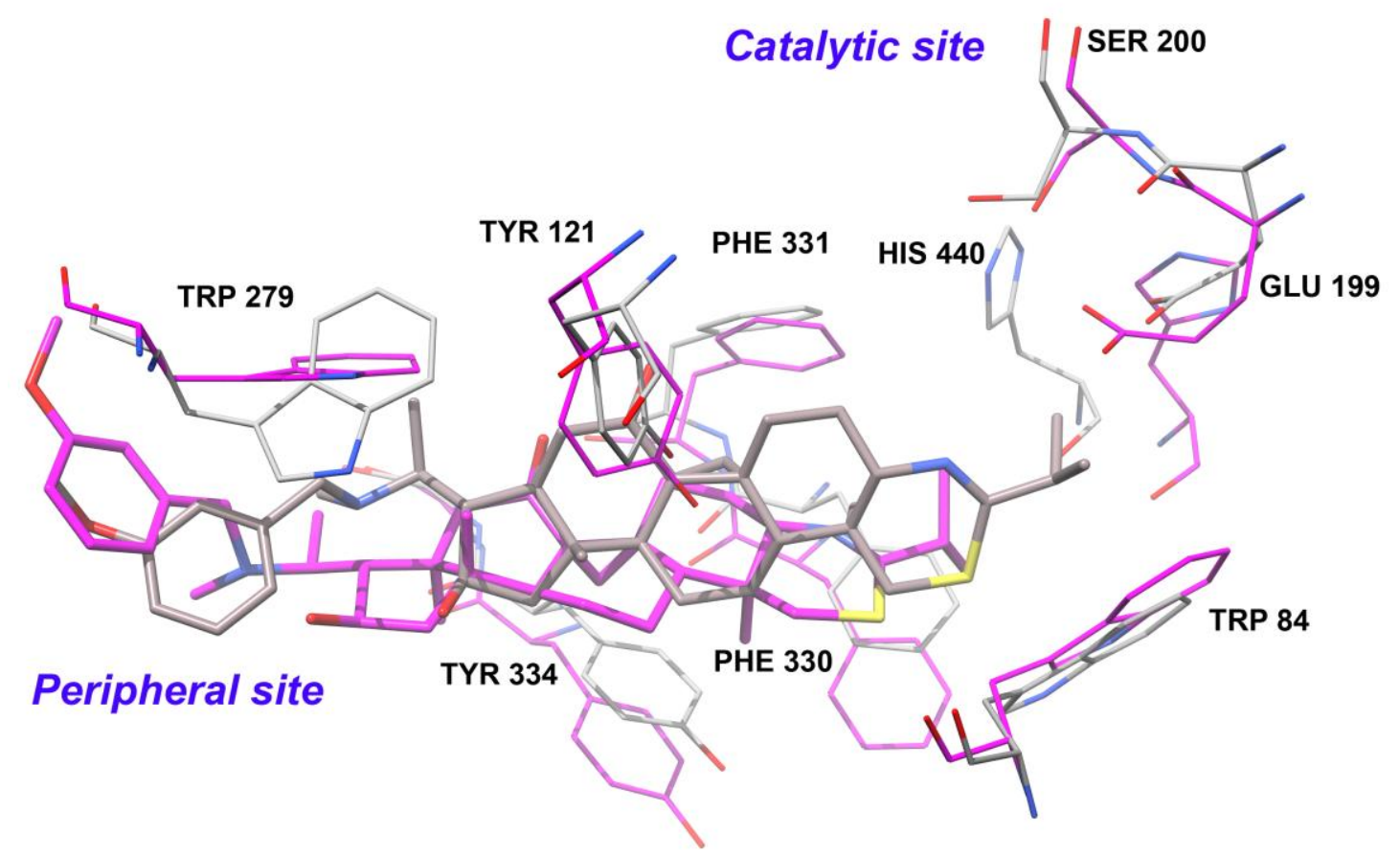

Figure 4 


\section{Figure legends}

Figure 1. Structures of compounds 1-3.

Figure 2. Selected residues of the protein binding site (apo form) at the beginning (gray) and at the end (orange) of the $10 \mathrm{~ns}$ molecular dynamics simulation

Figure 3. Selected residues of the protein binding site in complex with compound $\mathbf{1}$ at the beginning (gray) and at the end (green) of the $10 \mathrm{~ns}$ molecular dynamics simulation

Figure 4. Selected residues of the protein binding site in complex with compound 36e at the beginning (gray) and at the end (magenta) of the $10 \mathrm{~ns}$ molecular dynamics simulation

\section{Scheme legends}

Scheme 1. Synthesis of oxazines 9.

Scheme 2. Synthesis of tetrahydropyrimidines 13a-b.

Scheme 3. Synthesis of Dihydro- $1 H$-imidazole 20.

Scheme 4. Synthesis of dihydropyridine 25.

Scheme 5. Synthesis of tetrahydropyridine 27.

Scheme 6. Synthesis of 5,6-dihydro-4H-1,3-thiazine 29.

Scheme 7. Synthesis of compound 35a-c and 36a-k. 


\section{TABLES}

Table 1 : AChE and BuChE Inhibitory activities of new analogs. ${ }^{\text {a }}$

\begin{tabular}{ccccc}
\hline \multirow{2}{*}{ Compounds } & \multicolumn{3}{c}{$\mathrm{IC}_{50}(\mathrm{nM})$} & \\
\cline { 2 - 4 } & EeAChE & $h \mathrm{AChE}$ & AChE \\
selectivity $^{\mathrm{b}}$
\end{tabular}

${ }^{a}$ Values are expressed as the mean \pm standard error of the mean of three experiments. $\mathrm{IC}_{50}$ inhibitory concentration (nM) of AChE from Electrophorus electricus (EeAChE) or human recombinant ( $h \mathrm{AChE}$ ) or $\mathrm{BuChE}$ from human serum $(h \mathrm{BuChE}) .{ }^{\mathrm{b}} \mathrm{IC}_{50}(h \mathrm{BuChE}) / \mathrm{IC}_{50}(h \mathrm{AChE}) .{ }^{\mathrm{c}}$ Values from reference $8 .{ }^{\mathrm{d}}$ Not determined. 
Table 2. A $\beta$ aggregation of $36 \mathbf{e}, 36 \mathbf{h}$ and $36 \mathbf{k}$, propidium iodide and tacrine. ${ }^{\mathrm{a}}$

\begin{tabular}{|c|c|}
\hline compd & AChE-induced $\mathrm{A} \beta_{1-40}$ aggregation (\%) \\
\hline 36e & $69 \pm 2$ \\
\hline $36 h$ & $56 \pm 1$ \\
\hline $36 \mathbf{k}$ & $42 \pm 1$ \\
\hline propidium iodide & $82 \pm 2.5^{\mathrm{c}}$ \\
\hline tacrine & $7 \pm 0.21^{\mathrm{c}}$ \\
\hline
\end{tabular}


$>$ New acetylcholinesterase inhibitors in the triterpene series were synthesized. $>$

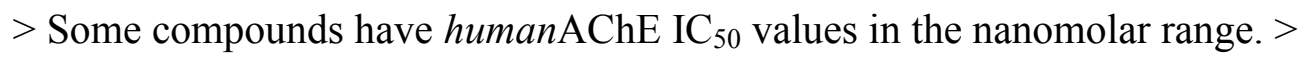

> Some analogs showed ability to block the AChE-induced A $\beta$ aggregation. $>$

$>$ A docking study and molecular dynamics simulations were done. $>$

> These compounds interact with both catalytic and peripheral sites of AChE. > 
Supplementary material

\title{
New Potent Human Acetylcholinesterase Inhibitors in the Tetracyclic Triterpene series with Inhibitory Potency on Amyloid $\beta$ Aggregation
}

\author{
Julien Rouleau, Bogdan I. Iorga and Catherine Guillou*. \\ Centre de Recherche de Gif, Institut de Chimie des Substances Naturelles, CNRS, \\ 1, Avenue de la Terrasse, 91198 Gif-sur-Yvette, France
}


$\begin{array}{ll}\text { Preparation of compound } \mathbf{6 b} & \text { S4 }\end{array}$

$\begin{array}{ll}\text { Preparation of compound } \mathbf{6 c} & \text { S5 }\end{array}$

$\begin{array}{ll}\text { Preparation of compound } \mathbf{7 b} & \text { S6 }\end{array}$

$\begin{array}{ll}\text { Preparation of compound 7c } & \text { S7 }\end{array}$

$\begin{array}{ll}\text { Preparation of compound } \mathbf{8 b} & \text { S8 }\end{array}$

$\begin{array}{lr}\text { Preparation of compound 8c } & \text { S9 }\end{array}$

$\begin{array}{lr}\text { Preparation of compound } \mathbf{9 b} & \text { S10 }\end{array}$

$\begin{array}{ll}\text { Preparation of compound 9c } & \text { S11 }\end{array}$

$\begin{array}{lr}\text { Preparation of compound 12a } & \text { S12 }\end{array}$

$\begin{array}{lr}\text { Preparation of compound 12b } & \text { S13 }\end{array}$

$\begin{array}{lr}\text { Preparation of compound } 14 & \text { S14 }\end{array}$

$\begin{array}{lr}\text { Preparation of compound } \mathbf{1 5} & \text { S15 }\end{array}$

$\begin{array}{ll}\text { Preparation of compound } \mathbf{1 6} & \text { S16 }\end{array}$

$\begin{array}{lr}\text { Preparation of compound } \mathbf{1 8} & \text { S17 }\end{array}$

$\begin{array}{lr}\text { Preparation of compound } 19 & \text { S18 }\end{array}$

$\begin{array}{lr}\text { Preparation of compound } 21 & \text { S19 }\end{array}$

$\begin{array}{lr}\text { Preparation of compound 22 } & \text { S20 }\end{array}$

$\begin{array}{ll}\text { Preparation of compound } \mathbf{2 3} & \text { S21 }\end{array}$

$\begin{array}{lr}\text { Preparation of compound } \mathbf{2 4} & \text { S22 }\end{array}$

$\begin{array}{ll}\text { Preparation of compound } 26 & \text { S23 }\end{array}$

$\begin{array}{lr}\text { Preparation of compound } 29 & \text { S24 }\end{array}$

$\begin{array}{ll}\text { Preparation of compound } \mathbf{3 1} & \text { S25 }\end{array}$

$\begin{array}{ll}\text { Preparation of compound } 32 & \text { S26 }\end{array}$

$\begin{array}{ll}\text { Preparation of compound } \mathbf{3 3} & \text { S27 }\end{array}$

$\begin{array}{lr}\text { Preparation of compound 35b } & \text { S28 }\end{array}$

$\begin{array}{lr}\text { Preparation of compound 35c } & \text { S29 }\end{array}$

$\begin{array}{lll}7 / 03 / 21 & 10: 03 & \text { S2 }\end{array}$ 
Preparation of compound 36a

Preparation of compound 36b

Preparation of compound 36c

Preparation of compound 36d

Preparation of compound 36f

Preparation of compound $\mathbf{3 6 g}$

Preparation of compound $\mathbf{3 6 h}$

Preparation of compound 36i

Preparation of compound 36j

Preparation of compound $\mathbf{3 6 k}$

Purity of tested compounds

Computer Aided Molecular Modeling

Figure S1, RMSD plot from MD simulations

Figure S2, RMSF plot from MD simulations

Figure S3, Protein-ligand contact surface plot from MD simulations

Figure S4, Equilibrium conformations for compounds $\mathbf{1}$ and 36e in the AChE binding site

Detailed experimental procedures and compound characterization data $\mathbf{6 b}-\mathbf{c}, \mathbf{7 b}-\mathbf{c}, \mathbf{8 b}-\mathbf{c}$, 9b-c, 12a-b, 14, 15, 16, 18, 19, 21, 22, 23, 24, 26, 29, 31, 32, 33, 35b-c, 36a-d, 36f-k. The assignement of the ${ }^{1} \mathrm{H}$ and ${ }^{13} \mathrm{C}$ NMR spectra of the different compounds has been made by ${ }^{1} \mathrm{H}-{ }^{1} \mathrm{H},{ }^{1} \mathrm{H}-{ }^{13} \mathrm{C}, \mathrm{COSY}, \mathrm{HMBC}, \mathrm{HMQC}$ and by analogy with previously described compounds. The numerotation indicated on molecule is only mentioned for the NMR spectra interpretation.

Note : The resonances of the CF3-group carbons are not being observed. The absence of such signals is ascribed to the slow relaxation times of these carbons and/or due to $13 \mathrm{C}-19 \mathrm{~F}$ couplings. Both of these phenomena are likely to lead to low-intensity signals for such carbons. The consequence of the free rotation of the $\mathrm{N}_{\mathrm{B}} \mathrm{Me}_{2}$ group is the apparition of a broad singlet in ${ }^{1} \mathrm{H}$ NMR and the diminution or disappearance of the corresponding peak in ${ }^{13} \mathrm{C}$ NMR. In some cases, this chemical shift can't be determined. 


\section{$N$-[20S-(dimethylamino)-16 $\alpha$-hydroxy-4 $\alpha$-(hydroxymethyl)-4 $\beta, 14 \alpha$-dimethyl-11-oxo-9,19- cyclo-5 $\alpha, 9 \beta$-pregnan-3 $\beta$-yl]-2-fluoro-2-methylpropanamide (6b)}

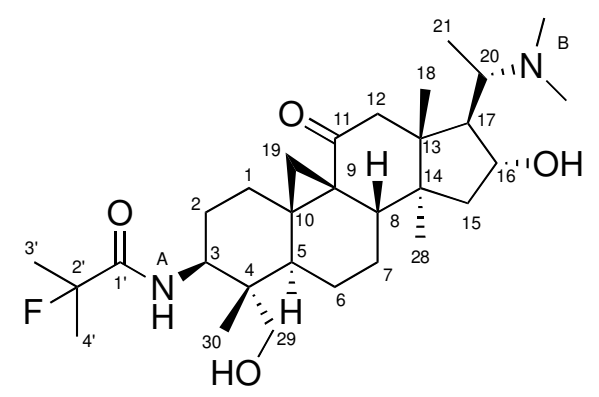

To $34 \mu \mathrm{L}\left(0.39 \mathrm{mmol}, 1.5\right.$ eq.) of oxalyl chloride solubilized in $1 \mathrm{~mL}$ of DCM at $0{ }^{\circ} \mathrm{C}$ were slowly added $30 \mu \mathrm{L}(0.39 \mathrm{mmol}, 1.5$ eq. $)$ of $\mathrm{DMF}$ and $30 \mu \mathrm{L}(0.34 \mathrm{mmol}, 1.3$ eq. $)$ of 2-fluoro-2-methylpropanoic acid. The mixture was stirred at $0{ }^{\circ} \mathrm{C}$ for $10 \mathrm{~min}$ and slowly added to $112 \mathrm{mg}$ ( $0.26 \mathrm{mmol}, 1$ eq.) of cycloxobuxidine-F 3 solubilized in $1.5 \mathrm{~mL}$ of pyridine and $1 \mathrm{~mL}$ of DCM at $-20^{\circ} \mathrm{C}$. The mixture was stirred at RT for $12 \mathrm{~h}$. After three co-evaporations with $3 \times 10 \mathrm{~mL}$ of 1,2-dichloroethane, the residue was dissolved in DCM, washed with a saturated solution of sodium carbonate and extracted with $3 \times 20 \mathrm{~mL}$ of DCM. Combined organic layers were washed with brine $(20 \mathrm{~mL})$, dried over magnesium sulfate and concentrated under vacuum. The crude product was purified by silica gel column chromatography (eluent : $\mathrm{DCM} / \mathrm{MeOH} / \mathrm{NH}_{4} \mathrm{OH}:$ : 96/3/1) to afford the title compound as an amorphous white solid (116 mg, yield $86 \%)$. IR $\left(\mathrm{cm}^{-1}\right): 3331,2936,2870,1659,1659,1535,1461,1161,1189,1225$; ${ }^{1} \mathrm{H}$ NMR $\left(300 \mathrm{MHz}, \mathrm{CDCl}_{3}\right): \delta(\mathrm{ppm}) 6.31\left(1 \mathrm{H}, \mathrm{dd}, \mathrm{J}_{\mathrm{NAH}-\mathrm{H} 3}=8.9 \mathrm{~Hz}, \mathrm{~J}_{\mathrm{NAH}-\mathrm{F}}=6.8 \mathrm{~Hz}, \mathrm{~N}_{\mathrm{A}}-\mathrm{H}\right)$, $4.07(2 \mathrm{H}, \mathrm{m}, \mathrm{H} 16 \beta+\mathrm{OH} 29), 3.95(1 \mathrm{H}, \mathrm{m}, \mathrm{H} 3 \alpha), 3.34(1 \mathrm{H}, \mathrm{m}, \mathrm{H} 29 \mathrm{~b}), 2.92\left(1 \mathrm{H}, \mathrm{d}, \mathrm{J}_{\mathrm{gem}}=11.9 \mathrm{~Hz}\right.$, $\mathrm{H} 29 \mathrm{a}), 2.60\left(1 \mathrm{H}, \mathrm{dq}, \mathrm{J}_{20-17 \alpha}=10.8 \mathrm{~Hz}, \mathrm{~J}_{20-21}=6.5 \mathrm{~Hz}, \mathrm{H} 20\right), 2.49\left(1 \mathrm{H}, \mathrm{d}, \mathrm{J}_{\text {gem }}=17.2 \mathrm{~Hz}, \mathrm{H} 12 \alpha\right)$, $2.42\left(1 \mathrm{H}, \mathrm{ddd}, \mathrm{J}_{\mathrm{gem}}=13.5 \mathrm{~Hz}, \mathrm{~J}_{1 \beta-2}=3.5 \mathrm{~Hz}, \mathrm{~J}_{1 \beta-2}=3.4 \mathrm{~Hz}, \mathrm{H} 1 \beta\right), 2.27\left(1 \mathrm{H}, \mathrm{d}, \mathrm{J}_{\mathrm{gem}}=17.2 \mathrm{~Hz}\right.$, $\mathrm{H} 12 \beta), 2.22\left(6 \mathrm{H}, \mathrm{bs}, \mathrm{N}_{\mathrm{B}} \mathrm{Me}_{2}\right), 1.92-2.07$ (4H, m, H5 $\left.\alpha+\mathrm{H} 17 \alpha+\mathrm{H} 15 \beta+\mathrm{H} 8 \beta\right), 1.64-1.71(3 \mathrm{H}, \mathrm{m}$, $\mathrm{H} 2 \alpha+\mathrm{H} 2 \beta+\mathrm{H} 6 \alpha), 1.59\left(3 \mathrm{H}, \mathrm{d}, \mathrm{J}_{\mathrm{H} 3^{\prime}-\mathrm{F}}=7.1 \mathrm{~Hz}, \mathrm{H} 3^{\prime}\right), 1.55(1 \mathrm{H}, \mathrm{m}, \mathrm{H} 19 \alpha), 1.52\left(3 \mathrm{H}, \mathrm{d}, \mathrm{J}_{\mathrm{H} 4^{\prime}-\mathrm{F}}=\right.$

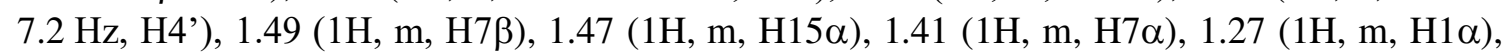
$1.19(3 \mathrm{H}, \mathrm{s}, \mathrm{H} 28), 1.02\left(1 \mathrm{H}, \mathrm{d}, \mathrm{J}_{\text {gem }}=3.7 \mathrm{~Hz}, \mathrm{H} 19 \beta\right), 0.88(1 \mathrm{H}, \mathrm{m}, \mathrm{H} 6 \beta), 0.84\left(3 \mathrm{H}, \mathrm{d}, \mathrm{J}_{21-20}=\right.$ $6.5 \mathrm{~Hz}, \mathrm{H} 21), 0.81(3 \mathrm{H}, \mathrm{s}, \mathrm{H} 18), 0.56(3 \mathrm{H}, \mathrm{s}, \mathrm{H} 30) ;{ }^{13} \mathrm{C} \mathrm{NMR}\left(75.4 \mathrm{MHz}, \mathrm{CDCl}_{3}\right): \delta(\mathrm{ppm})$ $211.3(\mathrm{C} 11), 174.4\left(\mathrm{~d}, \mathrm{~J}_{\mathrm{C} 1}{ }^{\prime} \mathrm{F}=20.9 \mathrm{~Hz}, \mathrm{C} 1^{\prime}\right), 96.1\left(\mathrm{~d}, \mathrm{~J}_{\mathrm{C}{ }^{\prime}-\mathrm{F}}=181.1 \mathrm{~Hz}, \mathrm{C} 2^{\prime}\right), 78.1(\mathrm{C} 16), 64.0$ (C29), 61.9 (C20), 55.7 (C17), 51.4 (C12), 50.6 (C3), 47.0 (C14), 44.6 (C4+C13), 42.7 (C15), 41.3 (C8), 41.1 (C5), 37.5 (C10), 34.3 (C9), 30.5 (C19), 27.5 (C1), 27.2 (C2), 25.5 (d, J $\mathrm{J}_{\mathrm{C} 3^{\prime}-\mathrm{F}}=$ $\left.23.6 \mathrm{~Hz}, \mathrm{C} 3^{\prime}\right), 24.6$ (d, J $\mathrm{J}^{\prime}$ - $\mathrm{F}=24.2 \mathrm{~Hz}, \mathrm{C}^{\prime}$ '), 24.2 (C7), 20.7 (C28), 18.3 (C6), 17.7 (C18), 11.1 (C30), $9.8(\mathrm{C} 21)$. MS (ESI, m/z) : $521.4(\mathrm{M}+\mathrm{H})$; HRMS (ESI, m/z) : calcd for $\mathrm{C}_{28} \mathrm{H}_{44} \mathrm{~N}_{2} \mathrm{O}_{4} \mathrm{~F}_{3}$ : 521.3755, found : 521.3755 . 


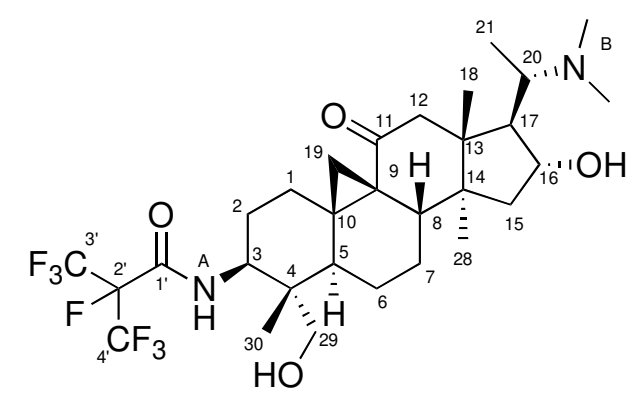

To $82 \mathrm{mg}(0.29 \mathrm{mmol}, 1$ eq.) of cyclobuxidine-F 3 solubilized in $4 \mathrm{~mL}$ of anhydrous $\mathrm{MeOH}$ were added $130 \mathrm{mg}(0.57 \mathrm{mmol}, 3 \mathrm{eq}$.) of methyl heptafluoroisobutyrate. The mixture was stirred at RT for $24 \mathrm{~h}$ and the solvent was evaporated under vacuum. The residue was purified by silica gel column chromatography (eluent : $\mathrm{DCM} / \mathrm{MeOH} / \mathrm{NH}_{4} \mathrm{OH}: 96 / 3 / 1$ ) to afford the title compound as an amorphous white solid (86 mg, yield $73 \%)$. IR $\left(\mathrm{cm}^{-1}\right): 3331,2936$, 2870, 1659, 1659, 1535, 1461, 1161, 1189, $1225 ;{ }^{1} \mathrm{H} \mathrm{NMR}\left(300 \mathrm{MHz}, \mathrm{CDCl}_{3}\right): \delta(\mathrm{ppm}) 6.69$ $\left(1 \mathrm{H}, \mathrm{dm}, \mathrm{J}_{\mathrm{NAH}-\mathrm{H} 3}=8.7 \mathrm{~Hz}, \mathrm{~N}_{\mathrm{A}}-\mathrm{H}\right), 4.16(1 \mathrm{H}, \mathrm{m}, \mathrm{H} 3 \alpha), 4.08(1 \mathrm{H}, \mathrm{m}, \mathrm{H} 16 \beta), 3.39(1 \mathrm{H}, \mathrm{m}, \mathrm{H} 29 \mathrm{~b})$, $2.96\left(1 \mathrm{H}, \mathrm{d}, \mathrm{J}_{\mathrm{gem}}=12.4 \mathrm{~Hz}, \mathrm{H} 29 \mathrm{a}\right), 2.62\left(1 \mathrm{H}, \mathrm{dq}, \mathrm{J}_{20-17 \alpha}=10.7 \mathrm{~Hz}, \mathrm{~J}_{20-21}=6.6 \mathrm{~Hz}, \mathrm{H} 20\right), 2.53$ $\left(1 \mathrm{H}, \mathrm{d}, \mathrm{J}_{\mathrm{gem}}=17.0 \mathrm{~Hz}, \mathrm{H} 12 \alpha\right), 2.48\left(1 \mathrm{H}, \mathrm{dt}, \mathrm{J}_{\mathrm{gem}}=13.4 \mathrm{~Hz}, \mathrm{~J}_{1 \beta-2}=3.5 \mathrm{~Hz}, \mathrm{H} 1 \beta\right), 2.28(1 \mathrm{H}, \mathrm{d}$, $\left.\mathrm{J}_{\text {gem }}=17.0 \mathrm{~Hz}, \mathrm{H} 12 \beta\right), 2.24\left(6 \mathrm{H}, \mathrm{bs}, \mathrm{N}_{\mathrm{B}} \mathrm{Me}_{2}\right), 2.09(2 \mathrm{H}, \mathrm{m}, \mathrm{H} 5 \alpha+\mathrm{H} 8 \beta), 2.00(1 \mathrm{H}, \mathrm{m}, \mathrm{H} 15 \beta)$, $1.98(1 \mathrm{H}, \mathrm{m}, \mathrm{H} 17 \alpha), 1.74(2 \mathrm{H}, \mathrm{m}, \mathrm{H} 2 \alpha+\mathrm{H} 2 \beta), 1.67(1 \mathrm{H}, \mathrm{m}, \mathrm{H} 6 \alpha), 1.56(1 \mathrm{H}, \mathrm{m}, \mathrm{H} 7 \beta), 1.54(1 \mathrm{H}$, $\left.\mathrm{d}, \mathrm{J}_{\mathrm{gem}}=3.8 \mathrm{~Hz}, \mathrm{H} 19 \alpha\right), 1.47(1 \mathrm{H}, \mathrm{m}, \mathrm{H} 15 \alpha), 1.41(1 \mathrm{H}, \mathrm{m}, \mathrm{H} 7 \alpha), 1.25(1 \mathrm{H}, \mathrm{m}, \mathrm{H} 1 \alpha), 1.21(3 \mathrm{H}$, s, H28), $1.06\left(1 \mathrm{H}, \mathrm{d}, \mathrm{J}_{\mathrm{gem}}=3.8 \mathrm{~Hz}, \mathrm{H} 19 \beta\right), 0.92(1 \mathrm{H}, \mathrm{m}, \mathrm{H} 6 \beta), 0.87\left(3 \mathrm{H}, \mathrm{d}, \mathrm{J}_{21-20}=6.6 \mathrm{~Hz}, \mathrm{H} 21\right)$, $0.85(3 \mathrm{H}, \mathrm{s}, \mathrm{H} 18), 0.62(3 \mathrm{H}, \mathrm{s}, \mathrm{H} 30) ;{ }^{13} \mathrm{C} \mathrm{NMR}\left(75.4 \mathrm{MHz}, \mathrm{CDCl}_{3}\right): \delta(\mathrm{ppm}) 211.0(\mathrm{C} 11), 158.8$ $\left(\mathrm{d}, \mathrm{J}_{\mathrm{C} 1}{ }^{\prime} \mathrm{F}=18.7 \mathrm{~Hz}, \mathrm{C} 1^{\prime}\right), 78.0(\mathrm{C} 16), 63.9(\mathrm{C} 29), 61.8(\mathrm{C} 20), 55.8$ (C17), 52.1 (C3), 51.2 (C12), 46.9 (C14), 44.5 (C13), 44.3 (C4), 42.6 (C15), 41.5 (C8), 41.1 (C5), 36.8 (C10), 34.1 (C9), 30.2 (C19), 27.3 (C1), 27.1 (C2), 24.2 (C7), 20.4 (C28), 18.2 (C6), 17.5 (C18), 10.8 (C30), 9.5 (C21). MS (ESI, m/z) : $629.3(\mathrm{M}+\mathrm{H})$; HRMS (ESI, m/z) : calcd for $\mathrm{C}_{30} \mathrm{H}_{44} \mathrm{~N}_{2} \mathrm{O}_{4} \mathrm{~F}_{7}: 629.3189$, found : 629.3158 . 


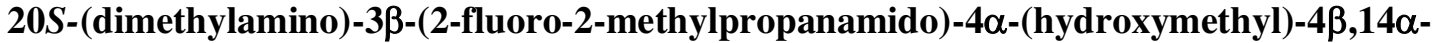 dimethyl-11-oxo-9,19-cyclo-5 $\alpha, 9 \beta$-pregnan-16 $\alpha$-yl acetate (7b)}

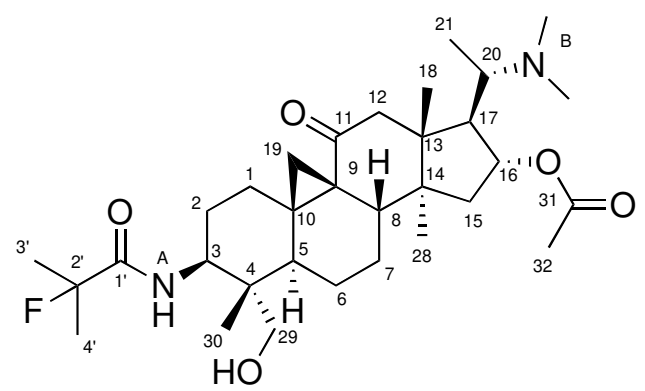

To $189 \mathrm{mg}(0.36 \mathrm{mmol}, 1 \mathrm{eq}$.) of $\mathbf{6 b}$ solubilized in $10 \mathrm{~mL}$ of a mixture DCM/pyridine (3/1) were added at $0{ }^{\circ} \mathrm{C} 41 \mu \mathrm{L}(0.43 \mathrm{mmol}, 1.2 \mathrm{eq}$.) of acetic anhydride. The mixture was stirred at $0{ }^{\circ} \mathrm{C}$ for $1 \mathrm{~h}$ then at RT for further $12 \mathrm{~h}$. After 3 co-evaporations with $3 \times 10 \mathrm{~mL}$ of $1,2-$ dichloroethane, the residue was dissolved in DCM, washed with $20 \mathrm{~mL}$ of a saturated solution of sodium hydrogen carbonate then extracted with $3 \times 20 \mathrm{~mL}$ of DCM. Combined organic layers were washed with brine $(20 \mathrm{~mL})$, dried over magnesium sulfate and concentrated under vacuum. The crude product was purified by silica gel column chromatography (eluent : DCM/MeOH/TEA : 96.5/3/0.5) to afford the title compound as an amorphous white solid (175 mg, yield $86 \%)$. IR $\left(\mathrm{cm}^{-1}\right): 3344,2970,2933,2873,2825,2733,1669,1652,1540,1456$, $1249,1229,1197 ;{ }^{1} \mathrm{H}$ NMR $\left(300 \mathrm{MHz}, \mathrm{CDCl}_{3}\right): \delta(\mathrm{ppm}) 6.29\left(1 \mathrm{H}, \mathrm{dd}, \mathrm{J}_{\mathrm{NAH}-\mathrm{H} 3}=8.7 \mathrm{~Hz}, \mathrm{~J}_{\mathrm{NAH}-\mathrm{F}}=\right.$ $\left.7.1 \mathrm{~Hz}, \mathrm{~N}_{\mathrm{A}}-\mathrm{H}\right), 5.13\left(1 \mathrm{H}, \mathrm{dd}, \mathrm{J}_{\mathrm{H} 16 \beta-\mathrm{H} 15 \mathrm{~b}}=8.1 \mathrm{~Hz}, \mathrm{~J}_{\mathrm{H} 16 \beta-\mathrm{H} 17 \mathrm{a}}=5.9 \mathrm{~Hz}, \mathrm{H} 16 \beta\right), 4.12(1 \mathrm{H}, \mathrm{dd}$, $\left.\mathrm{J}_{\mathrm{OH}-\mathrm{H} 29 \mathrm{~b}}=10.5 \mathrm{~Hz}, \mathrm{~J}_{\mathrm{OH}-\mathrm{H} 29 \mathrm{a}}=5.0 \mathrm{~Hz}, \mathrm{OH} 29\right), 3.99(1 \mathrm{H}, \mathrm{m}, \mathrm{H} 3 \alpha), 3.38\left(1 \mathrm{H}, \mathrm{dd}, \mathrm{J}_{\mathrm{gem}}=12.9 \mathrm{~Hz}\right.$, $\left.\mathrm{J}_{\mathrm{H} 29 \mathrm{~b}-\mathrm{OH}}=10.5 \mathrm{~Hz}, \mathrm{H} 29 \mathrm{~b}\right), 2.95\left(1 \mathrm{H}, \mathrm{dd}, \mathrm{J}_{\mathrm{gem}}=12.9 \mathrm{~Hz}, \mathrm{~J}_{\mathrm{H} 29 \mathrm{a}-\mathrm{OH}}=5.0 \mathrm{~Hz}, \mathrm{H} 29 \mathrm{a}\right), 2.55(1 \mathrm{H}, \mathrm{d}$, $\left.\mathrm{J}_{\mathrm{gem}}=17.2 \mathrm{~Hz}, \mathrm{H} 12 \alpha\right), 2.47(2 \mathrm{H}, \mathrm{m}, \mathrm{H} 20+\mathrm{H} 1 \beta), 2.39\left(1 \mathrm{H}, \mathrm{d}, \mathrm{J}_{\mathrm{gem}}=17.2 \mathrm{~Hz}, \mathrm{H} 12 \beta\right), 2.23(1 \mathrm{H}$, $\left.\mathrm{dd}, \mathrm{J}_{\mathrm{H} 17 \mathrm{a}-\mathrm{H} 20}=11.0 \mathrm{~Hz}, \mathrm{~J}_{\mathrm{H} 17 \mathrm{a}-\mathrm{H} 16 \mathrm{~b}}=5.9 \mathrm{~Hz}, \mathrm{H} 17 \alpha\right), 2.14\left(6 \mathrm{H}, \mathrm{bs}, \mathrm{N}_{\mathrm{B}} \mathrm{Me}_{2}\right), 1.98-2.10(3 \mathrm{H}, \mathrm{m}$, $\mathrm{H} 5 \alpha+\mathrm{H} 8 \beta+\mathrm{H} 15 \beta), 2.01(3 \mathrm{H}, \mathrm{s}, \mathrm{H} 32), 1.70(3 \mathrm{H}, \mathrm{m}, \mathrm{H} 2 \alpha+\mathrm{H} 2 \beta+\mathrm{H} 6 \alpha), 1.63\left(3 \mathrm{H}, \mathrm{d}, \mathrm{J}_{\mathrm{H} 3}{ }^{\prime} \mathrm{F}=\right.$ $\left.6.9 \mathrm{~Hz}, \mathrm{H} 3{ }^{\prime}\right), 1.59\left(1 \mathrm{H}, \mathrm{d}, \mathrm{J}_{\mathrm{gem}}=3.9 \mathrm{~Hz}, \mathrm{H} 19 \alpha\right), 1.56\left(3 \mathrm{H}, \mathrm{d}, \mathrm{J}_{\mathrm{H} 4^{\prime}-\mathrm{F}}=6.9 \mathrm{~Hz}, \mathrm{H} 4^{\prime}\right), 1.47(1 \mathrm{H}, \mathrm{m}$, $\mathrm{H} 7 \beta), 1.42\left(1 \mathrm{H}, \mathrm{d}, \mathrm{J}_{\mathrm{gem}}=14.1 \mathrm{~Hz}, \mathrm{H} 15 \alpha\right), 1.38(1 \mathrm{H}, \mathrm{m}, \mathrm{H} 7 \alpha), 1.26(1 \mathrm{H}, \mathrm{m}, \mathrm{H} 1 \alpha), 1.16(3 \mathrm{H}, \mathrm{s}$, $\mathrm{H} 28), 1.06\left(1 \mathrm{H}, \mathrm{d}, \mathrm{J}_{\mathrm{gem}}=3.9 \mathrm{~Hz}, \mathrm{H} 19 \beta\right), 0.85(3 \mathrm{H}, \mathrm{s}, \mathrm{H} 18), 0.82\left(3 \mathrm{H}, \mathrm{d}, \mathrm{J}_{\mathrm{H} 21-\mathrm{H} 20}=6.5 \mathrm{~Hz}, \mathrm{H} 21\right)$, $0.60(3 \mathrm{H}, \mathrm{s}, \mathrm{H} 30) ;{ }^{13} \mathrm{C} \mathrm{NMR}\left(75.4 \mathrm{MHz}, \mathrm{CDCl}_{3}\right): \delta(\mathrm{ppm}) 211.2(\mathrm{C} 11), 174.5\left(\mathrm{~d}, \mathrm{~J}_{\mathrm{Cl}}{ }^{\prime} \mathrm{F}=\right.$ $20.3 \mathrm{~Hz}, \mathrm{C} 1$ '), 170.6 (C31), 96.1 (d, J J $\mathrm{J}^{\prime}-\mathrm{F}=181.7 \mathrm{~Hz}, \mathrm{C} 2$ '), 78.8 (C16), 64.1 (C29), 59.4 (C20), 55.1 (C17), 51.9 (C12), 50.7 (C3), 47.4 (C14), 44.6 (C4), 44.6 (C13), 43.0 (C15), 41.4 (C8), 41.2 (C5), $40.2\left(\mathrm{~N}_{\mathrm{B}} \mathrm{Me}_{2}\right), 37.6$ (C10), 33.9 (C9), 30.7 (C19), 27.6 (C1), 27.4 (C2), 25.6 (d, $\mathrm{J}_{\mathrm{C} 3^{\prime}-\mathrm{F}}=24.2 \mathrm{~Hz}, \mathrm{C}^{\prime}$ '), 24.7 (d, $\mathrm{J}_{\mathrm{C}^{\prime}-\mathrm{F}}=24.2 \mathrm{~Hz}, \mathrm{C}^{\prime}$ '), $24.3(\mathrm{C} 7), 21.2(\mathrm{C} 32), 19.5(\mathrm{C} 28), 18.4$ (C6), 17.8 (C18), 11.1 (C30), 9.8 (C21) ; MS (ESI, m/z) : $563.3(\mathrm{M}+\mathrm{H}), 521.4$; HRMS (ESI, $\mathrm{m} / \mathrm{z}$ ) : calcd for $\mathrm{C}_{32} \mathrm{H}_{52} \mathrm{~N}_{2} \mathrm{O}_{5} \mathrm{~F}: 563.3860$, found : 563.3845 . 


\section{S-(dimethylamino)-3 $\beta$-(heptafluoroisobutanamino)-4 $\alpha$-(hydroxymethyl)-4 $\beta, 14 \alpha$ - dimethyl-11-oxo-9,19-cyclo-5 $\alpha, 9 \beta$-pregnan-16 $\alpha$-yl benzoate (7c)}

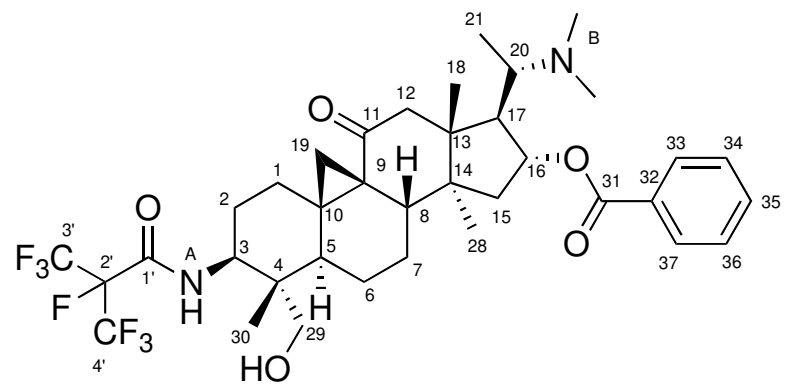

To $51 \mathrm{mg}(0.081 \mathrm{mmol}, 1$ eq. $)$ of $\mathbf{6 c}$ solubilized in $6 \mathrm{~mL}$ of a mixture DCM/pyridine (3/1) were added at $0{ }^{\circ} \mathrm{C} 23 \mathrm{mg}(0.092 \mathrm{mmol}, 1.2$ eq. $)$ of benzoic anhydride. The mixture was stirred at $0{ }^{\circ} \mathrm{C}$ for $1 \mathrm{~h}$ then at RT for further $12 \mathrm{~h}$. After 3 co-evaporations with $3 \times 10 \mathrm{~mL}$ of 1,2dichloroethane, the residue was dissolved in DCM, washed with $20 \mathrm{~mL}$ of a saturated solution of sodium carbonate then extracted with $3 \times 20 \mathrm{~mL}$ of DCM. Combined organic layers were washed with brine $(20 \mathrm{~mL})$, dried over magnesium sulfate and concentrated under vacuum. The crude product was purified by silica gel column chromatography (eluent : $\mathrm{DCM} / \mathrm{MeOH} / \mathrm{NH}_{4} \mathrm{OH}$ : 98/1/1) to afford the title compound as an amorphous white solid (55 mg, yield 95\%). IR ( $\left.\mathrm{cm}^{-1}\right)$ : $3538,3447,2969,2932,2862,2833,1708,1642,1523,1450,1259,1225,1128 ;{ }^{1} \mathrm{H}$ NMR $\left(300 \mathrm{MHz}, \mathrm{CDCl}_{3}\right): \delta(\mathrm{ppm}) 8.04(2 \mathrm{H} . \mathrm{d} . \mathrm{J}=7.2 \mathrm{~Hz}, \mathrm{H} 33+\mathrm{H} 37), 7.55(1 \mathrm{H}, \mathrm{m}, \mathrm{H} 35), 7.44(2 \mathrm{H}, \mathrm{m}$, $\mathrm{H} 34+\mathrm{H} 36), 6.62\left(1 \mathrm{H}, \mathrm{dd}, \mathrm{J}_{\mathrm{NAH}-\mathrm{H} 3}=8.9 \mathrm{~Hz}, \mathrm{~J}_{\mathrm{NAH}-\mathrm{F}}=2.7 \mathrm{~Hz}, \mathrm{~N}_{\mathrm{A}}-\mathrm{H}\right), 5.42\left(1 \mathrm{H}, \mathrm{dd}, \mathrm{J}_{\mathrm{H} 16 \beta-\mathrm{H} 15 \square}=\right.$ $\left.8.1 \mathrm{~Hz}, \mathrm{~J}_{\mathrm{H} 16 \beta-\mathrm{H} 17 \mathrm{\square}}=5.6 \mathrm{~Hz}, \mathrm{H} 16 \beta\right), 4.17(1 \mathrm{H}, \mathrm{m}, \mathrm{H} 3 \alpha), 3.39(1 \mathrm{H}, \mathrm{m}, \mathrm{H} 29 \mathrm{~b}), 2.96\left(1 \mathrm{H}, \mathrm{d}, \mathrm{J}_{\mathrm{gem}}=\right.$ $12.4 \mathrm{~Hz}, \mathrm{H} 29 \mathrm{a}), 2.63\left(1 \mathrm{H}, \mathrm{d}, \mathrm{J}_{\text {gem }}=17.1 \mathrm{~Hz}, \mathrm{H} 12 \alpha\right), 2.52(2 \mathrm{H}, \mathrm{m}, \mathrm{H} 20+\mathrm{H} 1 \beta), 2.46(1 \mathrm{H}, \mathrm{m}$, $\mathrm{H} 17 \alpha), 2.44\left(1 \mathrm{H}, \mathrm{d}, \mathrm{J}_{\mathrm{gem}}=17.1 \mathrm{~Hz}, \mathrm{H} 12 \beta\right), 1.96-2.11\left(9 \mathrm{H}, \mathrm{m}, \mathrm{N}_{\mathrm{B}} \mathrm{Me}_{2}+\mathrm{H} 5 \alpha+\mathrm{H} 8 \beta+\mathrm{H} 15 \beta\right), 1.77$ $(2 \mathrm{H}, \mathrm{m}, \mathrm{H} 2 \alpha+\mathrm{H} 2 \beta), 1.67(1 \mathrm{H}, \mathrm{m}, \mathrm{H} 6 \alpha), 1.55-1.60(2 \mathrm{H}, \mathrm{m}, \mathrm{H} 19 \alpha+\mathrm{H} 15 \alpha), 1.51(1 \mathrm{H}, \mathrm{m}, \mathrm{H} 7 \beta)$, $1.37(1 \mathrm{H}, \mathrm{m}, \mathrm{H} 7 \alpha), 1.30(1 \mathrm{H}, \mathrm{m}, \mathrm{H} 1 \alpha), 1.24(3 \mathrm{H}, \mathrm{s}, \mathrm{H} 28), 1.07\left(1 \mathrm{H}, \mathrm{d}, \mathrm{J}_{\mathrm{gem}}=3.8 \mathrm{~Hz}, \mathrm{H} 19 \beta\right)$, $0.95(1 \mathrm{H}, \mathrm{m}, \mathrm{H} 6 \beta), 0.85\left(3 \mathrm{H}, \mathrm{d}, \mathrm{J}_{21-20}=6.0 \mathrm{~Hz}, \mathrm{H} 21\right), 0.91(3 \mathrm{H}, \mathrm{s}, \mathrm{H} 18), 0.62(3 \mathrm{H}, \mathrm{s}, \mathrm{H} 30) ;{ }^{13} \mathrm{C}$ NMR (75.4 MHz, $\left.\mathrm{CDCl}_{3}\right)$ : $\delta(\mathrm{ppm}) 211.2(\mathrm{C} 11), 165.9(\mathrm{C} 31), 158.2\left(\mathrm{~d}, \mathrm{~J}_{\mathrm{Cl}}{ }^{\prime} \mathrm{F}=19.2 \mathrm{~Hz}, \mathrm{C} 1^{\prime}\right)$, 132.5 (C35), 130.8 (C32), 129.4 (C33+C37), 128.2 (C34+C36), 79.5 (C16), 63.8 (C29), 59.4 (C20), 55.3 (C17), 52.2 (C3), 51.8 (C12), 47.5 (C14), 44.8 (C13), 44.6 (C4), 43.2 (C15), 41.6 (C8), 41.2 (C5), $40.2\left(\mathrm{~N}_{\mathrm{B}} \mathrm{Me}_{2}\right), 37.2$ (C10), 33.9 (C9), 30.7 (C19), 27.4 (C1), 27.3 (C2), 24.3 (C7), 19.6 (C28), 18.4 (C6), 17.9 (C18), 11.1 (C30), 9.9 (C21) ; MS (ESI, m/z) : 733.3 (M+H) ; HRMS (ESI, m/z) : calcd for $\mathrm{C}_{37} \mathrm{H}_{48} \mathrm{~N}_{2} \mathrm{O}_{5} \mathrm{~F}_{7}:$ : 733.3494, found : 733.351 . 


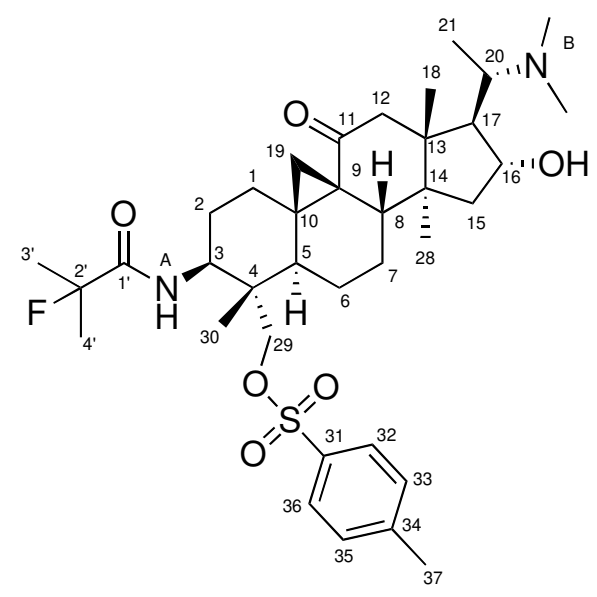

The title compound was prepared from $75 \mathrm{mg}(0.31 \mathrm{mmol}, 1$ eq.) of $7 \mathbf{b}, 10 \mathrm{~mL}$ of anhydrous $\mathrm{MeOH}, 60 \mathrm{mg}$ ( $0.31 \mathrm{mmol}, 1$ eq.) of PTSA, $1.5 \mathrm{~mL}$ of pyridine and $178 \mathrm{mg}$ $(0.93 \mathrm{mmol}, 3 \mathrm{eq}$.) of tosyl chloride by following the similar procedure described for the preparation of 8a. The crude product was purified by reverse phase silica gel column chromatography (eluent : water/EtOH/HCOOH : 80/20/0.1) to afford the title compound as an amorphous white solid (149 mg, yield $66 \%)$. IR $\left(\mathrm{cm}^{-1}\right): 3330,2962-2934,2869,1667,1598$, 1525, 1455, 1261, 1188, $1174 ;{ }^{1} \mathrm{H}$ NMR $\left(300 \mathrm{MHz}, \mathrm{CDCl}_{3}\right): \delta(\mathrm{ppm}) 7.81(2 \mathrm{H}, \mathrm{d}, \mathrm{J}=8.2 \mathrm{~Hz}$, $\mathrm{H} 32+\mathrm{H} 36), 7.33(2 \mathrm{H}, \mathrm{d}, \mathrm{J}=8.2 \mathrm{~Hz}, \mathrm{H} 33+\mathrm{H} 35), 6.07\left(1 \mathrm{H}, \mathrm{dd}, \mathrm{J}_{\mathrm{NAH}-\mathrm{H} 3}=9.9 \mathrm{~Hz}, \mathrm{~J}_{\mathrm{NAH}-\mathrm{F}}=7.0 \mathrm{~Hz}\right.$, $\left.\mathrm{N}_{\mathrm{A}} \mathrm{H}\right), 4.71(1 \mathrm{H}, \mathrm{t}, \mathrm{J}=7.5 \mathrm{~Hz}, \mathrm{H} 16 \beta), 4.11(1 \mathrm{H}, \mathrm{m}, \mathrm{H} 3 \alpha), 3.81\left(1 \mathrm{H}, \mathrm{d}, \mathrm{J}_{\mathrm{gem}}=9.8 \mathrm{~Hz}, \mathrm{H} 29 \mathrm{~b}\right)$, $3.57\left(1 \mathrm{H}, \mathrm{d}, \mathrm{J}_{\mathrm{gem}}=9.8 \mathrm{~Hz}, \mathrm{H} 29 \mathrm{a}\right), 3.42\left(1 \mathrm{H}, \mathrm{dq}, \mathrm{J}_{20-17 \alpha}=11.2 \mathrm{~Hz}, \mathrm{~J}_{20-21}=6.6 \mathrm{~Hz}, \mathrm{H} 20\right), 2.96(3 \mathrm{H}$, s, H5'), $2.84\left(3 \mathrm{H}, \mathrm{s}, \mathrm{H6}\right.$ '), $2.62\left(1 \mathrm{H}, \mathrm{d}, \mathrm{J}_{\mathrm{gem}}=17.0 \mathrm{~Hz}, \mathrm{H} 12 \alpha\right), 2.62(1 \mathrm{H}, \mathrm{s}, \mathrm{H} 37), 2.38(1 \mathrm{H}, \mathrm{m}$, $\mathrm{H} 1 \beta), 2.32\left(1 \mathrm{H}, \mathrm{d}, \mathrm{J}_{\mathrm{gem}}=17.0 \mathrm{~Hz}, \mathrm{H} 12 \beta\right), 2.27(1 \mathrm{H}, \mathrm{m}, \mathrm{H} 17 \alpha), 2.25\left(6 \mathrm{H}, \mathrm{bs}, \mathrm{N}_{\mathrm{B}} \mathrm{Me}_{2}\right), 2.09(1 \mathrm{H}$,

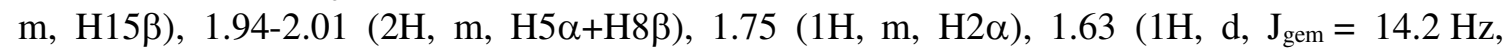
$\mathrm{H} 15 \alpha), 1.62(1 \mathrm{H}, \mathrm{m}, \mathrm{H} 7 \beta), 1.55(1 \mathrm{H}, \mathrm{m}, \mathrm{H} 19 \alpha), 1.51\left(3 \mathrm{H}, \mathrm{d}, \mathrm{J}_{\mathrm{H} 4^{\prime}-\mathrm{F}}=22.4 \mathrm{~Hz}, \mathrm{H} 4^{\prime}\right), 1.46(1 \mathrm{H}, \mathrm{m}$, $\mathrm{H} 2 \beta), 1.41(1 \mathrm{H}, \mathrm{m}, \mathrm{H} 6 \alpha), 1.39\left(3 \mathrm{H}, \mathrm{d}, \mathrm{J}_{\mathrm{H} 3^{\prime}-\mathrm{F}}=2.4 \mathrm{~Hz}, \mathrm{H} 3{ }^{\prime}\right), 1.26\left(3 \mathrm{H}, \mathrm{d}, \mathrm{J}_{21-20}=6.6 \mathrm{~Hz}, \mathrm{H} 21\right)$, $1.27(2 \mathrm{H}, \mathrm{m}, \mathrm{H} 7 \alpha+\mathrm{H} 1 \alpha), 1.06\left(1 \mathrm{H}, \mathrm{d}, \mathrm{J}_{\mathrm{gem}}=4.1 \mathrm{~Hz}, \mathrm{H} 19 \beta\right), 0.93(1 \mathrm{H}, \mathrm{m}, \mathrm{H} 6 \beta), 0.88(3 \mathrm{H}, \mathrm{s}$, $\mathrm{H} 18), 0.74(3 \mathrm{H}, \mathrm{s}, \mathrm{H} 30) ;{ }^{13} \mathrm{C}$ NMR $\left(75.4 \mathrm{MHz}, \mathrm{CDCl}_{3}\right)$ : $\delta(\mathrm{ppm}) 209.6(\mathrm{C} 11), 172.3\left(\mathrm{~d}, \mathrm{~J}_{\mathrm{C} 1} \cdot \mathrm{F}=\right.$ $19.8 \mathrm{~Hz}, \mathrm{Cl}$ '), 144.7 (C34), 132.6 (C31), 129.8 (C33+C35), 128.1 (C32+C36), 96.6 (d, J $\mathrm{J}_{\mathrm{C} 2}$ - $\mathrm{F}=$ $184.3 \mathrm{~Hz}, \mathrm{C} 2$ '), 73.4 (C16), 70.5 (C29), 65.1 (C20), 55.4 (C17), 51.7 (C12), 49.5 (C3), 47.4 (C14), 45.9 (C13), 45.5 (C15), 44.0 (C5'), 43.1 (C4), 42.6 (C8), 41.5 (C5), 37.5 (C10), 36.1 (C6'), 33.7 (C9), 30.8 (C19), $28.0(\mathrm{C} 1), 27.2(\mathrm{C} 2), 25.1$ (d, J $\left.\mathrm{C}^{\prime}-\mathrm{F}=18.4 \mathrm{~Hz}, \mathrm{C} 3^{\prime}\right), 24.8$ (d, J $\mathrm{J}_{\mathrm{C}{ }^{\prime}-\mathrm{F}}=$ 18.4 Hz, C4'), 24.3 (C7), 21.6 (C37), 20.0 (C28), 18.5 (C6), 17.9 (C18), 11.6 (C21), 10.9 (C30) ; MS (ESI, m/z) : $675.3(\mathrm{M}+\mathrm{H}) ; \mathrm{HRMS}(\mathrm{ESI}, \mathrm{m} / \mathrm{z})$ : calcd for $\mathrm{C}_{37} \mathrm{H}_{56} \mathrm{~N}_{2} \mathrm{O}_{6} \mathrm{FS}: 675.3843$, found : 675.3879 . 


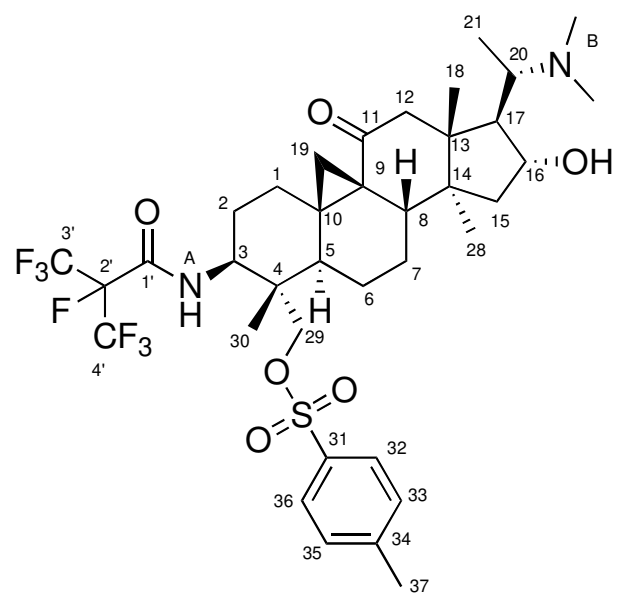

The title compound was prepared from $280 \mathrm{mg}(0.42 \mathrm{mmol}, 1 \mathrm{eq}$.$) of 7 \mathrm{c}, 20 \mathrm{~mL}$ of anhydrous $\mathrm{MeOH}, 80 \mathrm{mg}(0.42 \mathrm{mmol}, 1 \mathrm{eq}$.) of $p$-toluenesulfonic acid, $2.5 \mathrm{~mL}$ of pyridine, $398 \mathrm{mg}$ ( $2.1 \mathrm{mmol}, 5 \mathrm{eq}$.) of tosyl chloride by following similar procedure described for the preparation of 8a. The basic hydrolysis of benzoyl group was proceed at $60{ }^{\circ} \mathrm{C}$ for $3 \mathrm{~h}$. The crude product was purified by reverse phase silica gel column chromatography (eluent : water/ $\left.\mathrm{CH}_{3} \mathrm{CN} / \mathrm{HCOOH}: 75 / 25 / 0.1\right)$ to afford the title compound as an amorphous light yellow solid (101 mg, yield $31 \%$ ). IR $\left(\mathrm{cm}^{-1}\right): 3342,2932,2873,1716,1670,1539,1457,1236,1176$; ${ }^{1} \mathrm{H}$ NMR $\left(300 \mathrm{MHz}, \mathrm{CDCl}_{3}\right): \delta(\mathrm{ppm}) 7.78(2 \mathrm{H}, \mathrm{d}, \mathrm{J}=8.3 \mathrm{~Hz}, \mathrm{H} 33+\mathrm{H} 35), 7.78(2 \mathrm{H}, \mathrm{d}, \mathrm{J}=$ $8.3 \mathrm{~Hz}, \mathrm{H} 32+\mathrm{H} 36), 6.16\left(1 \mathrm{H}, \mathrm{dd}, \mathrm{J}_{\mathrm{NAH}-\mathrm{H} 3}=9.8 \mathrm{~Hz}, \mathrm{~J}_{\mathrm{NAH}-\mathrm{F}}=3.8 \mathrm{~Hz}, \mathrm{~N}_{\mathrm{A}}-\mathrm{H}\right), 4.21(1 \mathrm{H}, \mathrm{m}, \mathrm{H} 16 \beta)$, $4.13(1 \mathrm{H}, \mathrm{m}, \mathrm{H} 3 \alpha), 3.88\left(1 \mathrm{H}, \mathrm{d}, \mathrm{J}_{\mathrm{gem}}=10.1 \mathrm{~Hz}, \mathrm{H} 29 \mathrm{~b}\right), 3.40\left(1 \mathrm{H}, \mathrm{d}, \mathrm{J}_{\mathrm{gem}}=10.1 \mathrm{~Hz}, \mathrm{H} 29 \mathrm{a}\right), 2.65$ $\left(1 \mathrm{H}, \mathrm{dq}, \mathrm{J}_{20-17 \alpha}=10.8 \mathrm{~Hz}, \mathrm{~J}_{20-21}=6.5 \mathrm{~Hz}, \mathrm{H} 20\right), 2.53\left(1 \mathrm{H}, \mathrm{d}, \mathrm{J}_{\mathrm{gem}}=17.0 \mathrm{~Hz}, \mathrm{H} 12 \alpha\right), 2.48(1 \mathrm{H}, \mathrm{m}$, $\mathrm{H} 1 \beta), 2.44(3 \mathrm{H}, \mathrm{s}, \mathrm{H} 37), 2.31\left(1 \mathrm{H}, \mathrm{d}, \mathrm{J}_{\mathrm{gem}}=17.0 \mathrm{~Hz}, \mathrm{H} 12 \beta\right), 1.96-2.06(10 \mathrm{H}, \mathrm{m}$, $\left.\mathrm{H} 17 \alpha+\mathrm{N}_{\mathrm{B}} \mathrm{Me}_{2}+\mathrm{H} 5 \alpha+\mathrm{H} 8 \beta+\mathrm{H} 15 \beta\right), 1.78(1 \mathrm{H}, \mathrm{m}, \mathrm{H} 2 \alpha), 1.63(1 \mathrm{H}, \mathrm{m}, \mathrm{H} 2 \beta), 1.48-1.56(4 \mathrm{H}, \mathrm{m}$, $\mathrm{H} 6 \alpha+\mathrm{H} 19 \alpha+\mathrm{H} 15 \alpha+\mathrm{H} 7 \beta), 1.31(1 \mathrm{H}, \mathrm{m}, \mathrm{H} 7 \alpha), 1.30(1 \mathrm{H}, \mathrm{m}, \mathrm{H} 1 \alpha), 1.20(3 \mathrm{H}, \mathrm{s}, \mathrm{H} 28), 1.02(1 \mathrm{H}$, $\left.\mathrm{d}, \mathrm{J}_{\mathrm{gem}}=4.0 \mathrm{~Hz}, \mathrm{H} 19 \beta\right), 0.93(1 \mathrm{H}, \mathrm{m}, \mathrm{H} 6 \beta), 0.88\left(3 \mathrm{H}, \mathrm{d}, \mathrm{J}_{21-20}=6.5 \mathrm{~Hz}, \mathrm{H} 21\right), 0.84(3 \mathrm{H}, \mathrm{s}, \mathrm{H} 18)$, $0.75(3 \mathrm{H}, \mathrm{s}, \mathrm{H} 30) ;{ }^{13} \mathrm{C} \mathrm{NMR}\left(75.4 \mathrm{MHz}, \mathrm{CDCl}_{3}\right): \delta(\mathrm{ppm}) 211.2(\mathrm{C} 11), 156.1\left(\mathrm{~d}, \mathrm{~J}_{\mathrm{Cl}} \cdot \mathrm{F}=\right.$ $18.7 \mathrm{~Hz}, \mathrm{C1}$ '), 144.9 (C34), 132.3 (C31), 129.4 (C33+C35), 128.0 (C32+C36), 78.0 (C16), 70.1 (C29), 62.1 (C20), 55.7 (C17), 51.4 (C12), 51.3 (C3), 46.9 (C14), 44.4 (C13), 43.2 (C4), 42.9 (C15), 42.2 (C8), 42.0 (C5), 36.6 (C10), 34.0 (C9), 30.5 (C19), 27.8 (C1), 27.0 (C2), 24.3 (C7), 21.5 (C37), 20.9 (C28), 18.5 (C6), 17.9 (C18), 11.0 (C30), 10.0 (C21) ; MS (ESI, m/z) : 783.3 $(\mathrm{M}+\mathrm{H})$; HRMS (ESI, m/z) : calcd for $\mathrm{C}_{37} \mathrm{H}_{50} \mathrm{~N}_{2} \mathrm{O}_{6} \mathrm{~F}_{7} \mathrm{~S}: 783.3278$, found : 783.3328 . 


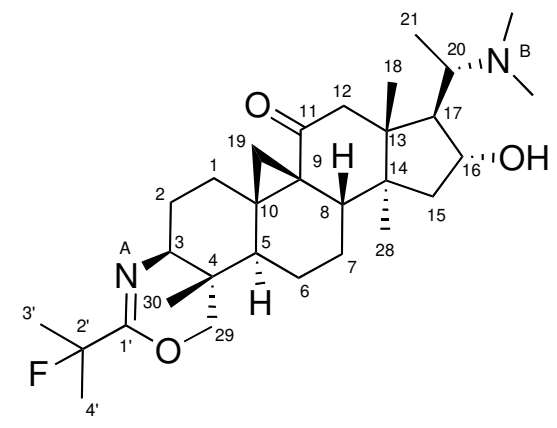

To $32 \mathrm{mg}$ ( $0.044 \mathrm{mmol}, 1$ eq.) of $\mathbf{8 b}$ solubilized in $3 \mathrm{~mL}$ of anhydrous DMF were added $21 \mathrm{mg}\left(0.22 \mathrm{mmol}, 5 \mathrm{eq}\right.$.) of sodium diformylamide. The mixture was stirred at $120{ }^{\circ} \mathrm{C}$ for $5 \mathrm{~h}$, washed with $20 \mathrm{~mL}$ of a sodium hydrogen carbonate saturated solution and extracted with $5 \times 10 \mathrm{~mL}$ of DCM. Combined organic layers were washed with brine, dried over magnesium sulfate and concentrated under vacuum. The crude product was purified by neutral alumina column chromatography (eluent : $\mathrm{DCM} / \mathrm{MeOH}: 7 / 3$ ) to afford the title compound as an amorphous colourless solid (7 mg, yield $32 \%)$. IR $\left(\mathrm{cm}^{-1}\right): 3329,2934,2869,1669,1456,1157$; ${ }^{1} \mathrm{H}$ NMR $\left(500 \mathrm{MHz}, \mathrm{CDCl}_{3}\right): \delta(\mathrm{ppm}) 4.10(1 \mathrm{H}, \mathrm{m}, \mathrm{H} 16 \beta), 4.03\left(1 \mathrm{H}, \mathrm{d}, \mathrm{J}_{\mathrm{gem}}=9.9 \mathrm{~Hz}, \mathrm{H} 29 \mathrm{~b}\right)$, $3.86\left(1 \mathrm{H}, \mathrm{d}, \mathrm{J}_{\mathrm{gem}}=9.9 \mathrm{~Hz}, \mathrm{H} 29 \mathrm{a}\right), 3.04(1 \mathrm{H}, \mathrm{dm}, \mathrm{J}=12.1 \mathrm{~Hz}, \mathrm{H} 3 \alpha), 2.62(1 \mathrm{H}, \mathrm{m}, \mathrm{H} 20), 2.50$ $\left(1 \mathrm{H}, \mathrm{d}, \mathrm{J}_{\mathrm{gem}}=17.0 \mathrm{~Hz}, \mathrm{H} 12 \alpha\right), 2.37(1 \mathrm{H}, \mathrm{m}, \mathrm{H} 2 \alpha), 2.33\left(1 \mathrm{H}, \mathrm{d}, \mathrm{J}_{\mathrm{gem}}=17.0 \mathrm{~Hz}, \mathrm{H} 12 \beta\right), 2.25(6 \mathrm{H}$, bs, $\left.\mathrm{N}_{\mathrm{B}} \mathrm{Me}_{2}\right), 2.13(1 \mathrm{H}, \mathrm{m}, \mathrm{H} 5 \alpha), 2.03(1 \mathrm{H}, \mathrm{m}, \mathrm{H} 15 \beta), 1.93-1.98(2 \mathrm{H}, \mathrm{m}, \mathrm{H} 17 \alpha+\mathrm{H} 1 \beta), 1.67(1 \mathrm{H}$, $\left.\mathrm{d}, \mathrm{J}_{\mathrm{gem}}=4.1 \mathrm{~Hz}, \mathrm{H} 19 \alpha\right), 1.50-1.59\left(10 \mathrm{H}, \mathrm{m}, \mathrm{H} 7 \beta+\mathrm{H} 3^{\prime}+\mathrm{H} 4{ }^{\prime}+\mathrm{H} 15 \alpha+\mathrm{H} 1 \alpha+\mathrm{H} 8 \beta\right), 1.30(1 \mathrm{H}, \mathrm{m}$, $\mathrm{H} 2 \beta), 1.22(2 \mathrm{H}, \mathrm{m}, \mathrm{H} 6 \alpha+\mathrm{H} 7 \alpha), 1.10\left(1 \mathrm{H}, \mathrm{d}, \mathrm{J}_{\mathrm{gem}}=4.1 \mathrm{~Hz}, \mathrm{H} 19 \beta\right), 0.92(1 \mathrm{H}, \mathrm{m}, \mathrm{H} 6 \beta), 0.88(3 \mathrm{H}$, d, $\left.\mathrm{J}_{21-20}=6.1 \mathrm{~Hz}, \mathrm{H} 21\right), 0.84(3 \mathrm{H}, \mathrm{s}, \mathrm{H} 18), 0.82(3 \mathrm{H}, \mathrm{s}, \mathrm{H} 30) ;{ }^{13} \mathrm{C} \mathrm{NMR}\left(125.6 \mathrm{MHz}, \mathrm{CDCl}_{3}\right)$ : $\delta(\mathrm{ppm}) 210.5$ (C11), 158.8 (C1'), 93.7 (C2'), 78.3 (C16), 76.5 (C29), 62.1 (C20), 59.6 (C3), 55.9 (C17), 51.4 (C12), 47.2 (C14), 46.2 (C8), 44.7 (C13), 42.6 (C15), 40.7 (C5), 37.1 (C10), 34.9 (C9), 34.1 (C4), 29.7 (C19), 28.4 (C2), 28.2 (C1), 25.5 (d, J $\left.\mathrm{J}^{\prime}{ }^{\prime}-\mathrm{F}=23.8 \mathrm{~Hz}, \mathrm{C} 3^{\prime}\right), 25.4$ (d, $\mathrm{J}_{\mathrm{C} 4^{\prime}-\mathrm{F}}=23.8 \mathrm{~Hz}, \mathrm{C}^{\prime}$ ) $), 24.3(\mathrm{C} 7), 20.5$ (C28), 18.1 (C6), 17.9 (C18), 10.9 (C30), 10.0 (C21) ; MS $\left(\right.$ ESI, m/z) : $503.3(\mathrm{M}+\mathrm{H})$; HRMS (ESI, m/z) : calcd for $\mathrm{C}_{30} \mathrm{H}_{48} \mathrm{~N}_{2} \mathrm{O}_{3} \mathrm{~F}: 503.3649$, found : 503.3642 . 


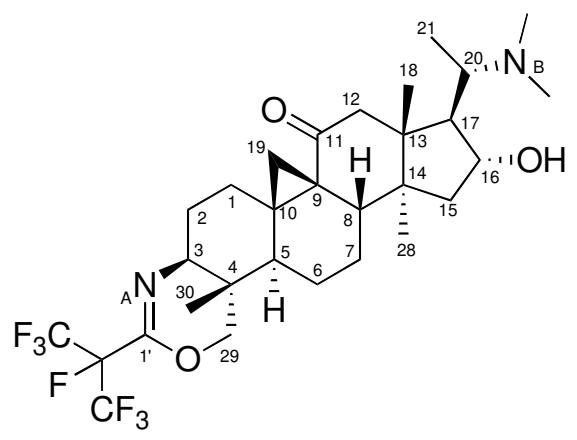

$28.5 \mathrm{mg}(0.036 \mathrm{mmol}, 1$ eq. $)$ of $\mathbf{8 c}, 17 \mathrm{mg}(0.182 \mathrm{mmol}, 5$ eq.) of sodium diformylamide and $2 \mathrm{~mL}$ of DMF were used by following similar procedure described for the preparation of $9 \mathbf{a}$. $3 \mathrm{mg}$ of $9 \mathrm{c}$ were isolated as amorphous white solid (14\%).

IR $\left(\mathrm{cm}^{-1}\right): 3400,2953,2924,2857,1734,1688,1672,1460,1270,1236 ;{ }^{1} \mathrm{H}$ NMR $(500 \mathrm{MHz}$, $\left.\mathrm{CDCl}_{3}\right): \delta(\mathrm{ppm}) 4.16\left(1 \mathrm{H}, \mathrm{d}, \mathrm{J}_{\mathrm{gem}}=10.1 \mathrm{~Hz}, \mathrm{H} 29 \mathrm{a}\right), 4.10(1 \mathrm{H}, \mathrm{m}, \mathrm{H} 16 \beta), 4.00\left(1 \mathrm{H}, \mathrm{d}, \mathrm{J}_{\mathrm{gem}}=\right.$ $10.1 \mathrm{~Hz}, \mathrm{H} 29 \mathrm{~b}), 3.20(1 \mathrm{H}, \mathrm{m}, \mathrm{H} 3 \alpha), 2.63(1 \mathrm{H}, \mathrm{dq}, \mathrm{J}=11.0 \mathrm{~Hz}, \mathrm{~J}=6.4 \mathrm{~Hz}, \mathrm{H} 20), 2.58(1 \mathrm{H}, \mathrm{d}$, $\left.\mathrm{J}_{\mathrm{gem}}=17.1 \mathrm{~Hz}, \mathrm{H} 12 \alpha\right), 2.41(1 \mathrm{H}, \mathrm{m}, \mathrm{H} 1 \beta), 2.35(1 \mathrm{H}, \mathrm{d}, \mathrm{J}=17.1 \mathrm{~Hz}, \mathrm{H} 12 \beta), 2.42(6 \mathrm{H}, \mathrm{bs}$,

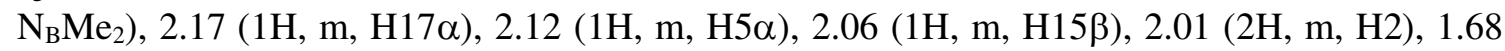
$\left(1 \mathrm{H}, \mathrm{d}, \mathrm{J}_{\mathrm{gem}}=4.0 \mathrm{~Hz}, \mathrm{H} 19 \alpha\right), 1.58(1 \mathrm{H}, \mathrm{m}, \mathrm{H} 15 \alpha), 1.56(1 \mathrm{H}, \mathrm{m}, \mathrm{H} 8 \beta), 1.14(1 \mathrm{H}, \mathrm{m}, \mathrm{H} 6 \alpha), 1.34$ $(2 \mathrm{H}, \mathrm{m}, \mathrm{H} 7), 1.59(1 \mathrm{H}, \mathrm{m}, \mathrm{H} 1 \alpha), 1.22(3 \mathrm{H}, \mathrm{s}, \mathrm{H} 28), 1.12\left(1 \mathrm{H}, \mathrm{d}, \mathrm{J}_{\mathrm{gem}}=4.0 \mathrm{~Hz}, \mathrm{H} 19 \beta\right), 0.88(3 \mathrm{H}$, $\mathrm{d}, \mathrm{J}=6.4 \mathrm{~Hz}, \mathrm{H} 21), 0.86(3 \mathrm{H}, \mathrm{s}, \mathrm{H} 18), 0.85(1 \mathrm{H}, \mathrm{m}, \mathrm{H} 6 \beta), 0.84(3 \mathrm{H}, \mathrm{m}, \mathrm{H} 30) ;{ }^{13} \mathrm{C}$ NMR (125.6 $\left.\mathrm{MHz}, \mathrm{CDCl}_{3}\right)$ : $\delta(\mathrm{ppm}) 209.4$ (C11), 144.6 (C1'), 78.5 (C16), 77.8 (C29), 60.4 (C3), 62.1 (C20), 56.0 (C17), 51.6 (C12), 47.1 (C14), 45.8 (C8), 44.7 (C13), 44.2 (C15), 40.4 (C5), 36.4 (C10), 35.2 (C9), 34.9 (C4), 29.8 (C19), 28.1 (C1), 27.3 (C2), 22.3 (C7), 19.7 (C28), 19.3 (C6), 17.7 (C18), $11.0(\mathrm{C} 21), 10.6(\mathrm{C} 30)$; MS (ESI, m/z) : $611.3(\mathrm{M}+\mathrm{H})$; HRMS (ESI, m/z) : calcd for $\mathrm{C}_{30} \mathrm{H}_{42} \mathrm{~F}_{7} \mathrm{~N}_{2} \mathrm{O}_{3}: 611.3085$, found : 611.3085 . 


\section{$N$-[20S-(dimethylamino)-16 $\alpha$-hydroxy-4 $\beta, 14 \alpha$-dimethyl-4 $\alpha$-[(methylamino)methyl]-11-oxo- 9,19-cyclo-5 $\alpha, 9 \beta$-pregnan-3 $\beta$-yl]-isobutyramide (12a)}

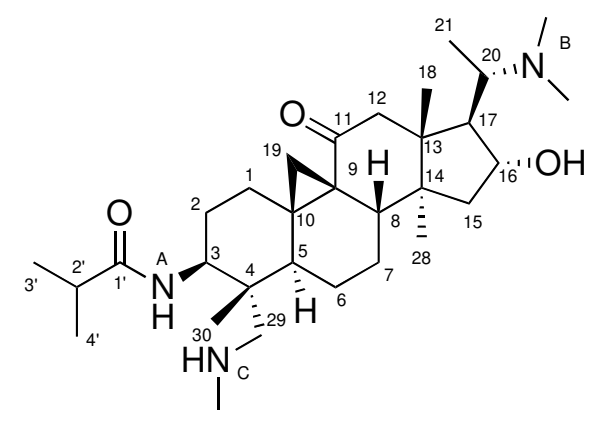

To $100 \mathrm{mg}(0.19 \mathrm{mmol}, 1$ eq.) of aldehyde $\mathbf{1 0}$ solubilized in $1 \mathrm{~mL}$ of EtOH were added $100 \mu \mathrm{L}$ (1.4 mmol, 7 eq.) of methylamine ( $33 \%$ in EtOH) and a small amount of sodium sulfate. The mixture was stirred at $60{ }^{\circ} \mathrm{C}$ for $12 \mathrm{~h}$, filtered and concentrated under vacuum. The formation of imine was controlled by NMR. The residue was dissolved in $1 \mathrm{~mL}$ of EtOH and $34 \mu \mathrm{L}$ ( $0.6 \mathrm{mmol}, 3 \mathrm{eq}$.) of acetic acid and $19 \mathrm{mg}(0.3 \mathrm{mmol}, 1.5 \mathrm{eq}$.$) of sodium$ cyanoborohydride were added at $0{ }^{\circ} \mathrm{C}$. The mixture was stirred at RT for $1 \mathrm{~h}$, washed with $20 \mathrm{~mL}$ of a saturated sodium carbonate solution then extracted with $3 \times 20 \mathrm{~mL}$ of DCM. Combined organic layers were washed with brine, dried over magnesium sulfate and concentrated under vacuum to afford the title compound without further purification as an amorphous white solide (93 mg, yield $90 \%)$. IR $\left(\mathrm{cm}^{-1}\right): 3308,2961,2931,2869,2839,1732$, $1649,1537,1450,1226,1188,1156,1095 ;{ }^{1} \mathrm{H}$ NMR $\left(300 \mathrm{MHz}, \mathrm{CDCl}_{3}\right): \delta(\mathrm{ppm}) 5.46(1 \mathrm{H}, \mathrm{d}$, $\left.\mathrm{J}_{\mathrm{NAH}-3}=9.7 \mathrm{~Hz}, \mathrm{~N}_{\mathrm{A}}-\mathrm{H}\right), 4.10(1 \mathrm{H}, \mathrm{m}, \mathrm{H} 16 \beta), 3.97(1 \mathrm{H}, \mathrm{m}, \mathrm{H} 3 \alpha), 2.62\left(1 \mathrm{H}, \mathrm{dq}, \mathrm{J}_{20-17 \alpha}=10.7 \mathrm{~Hz}\right.$, $\left.\mathrm{J}_{20-21}=6.5 \mathrm{~Hz}, \mathrm{H} 20\right), 2.51\left(1 \mathrm{H}, \mathrm{d}, \mathrm{J}_{\mathrm{gem}}=17.2 \mathrm{~Hz}, \mathrm{H} 12 \alpha\right), 2.44(1 \mathrm{H}, \mathrm{m}, \mathrm{H} 1 \beta), 2.36(1 \mathrm{H}, \mathrm{m}, \mathrm{H} 2$ '), $2.34(3 \mathrm{H}, \mathrm{s}, \mathrm{H} 31), 2.27\left(1 \mathrm{H}, \mathrm{d}, \mathrm{J}_{\mathrm{gem}}=17.2 \mathrm{~Hz}, \mathrm{H} 12 \beta\right), 2.25\left(6 \mathrm{H}, \mathrm{bs}, \mathrm{N}_{\mathrm{B}} \mathrm{Me}_{2}\right), 2.21(2 \mathrm{H}, \mathrm{m}, \mathrm{H} 29)$, $2.12(1 \mathrm{H}, \mathrm{m}, \mathrm{H} 5 \alpha), 2.02(1 \mathrm{H}, \mathrm{m}, \mathrm{H} 15 \beta), 1.98(1 \mathrm{H}, \mathrm{m}, \mathrm{H} 17 \alpha), 1.91(1 \mathrm{H}, \mathrm{m}, \mathrm{H} 8 \beta), 1.68(1 \mathrm{H}, \mathrm{m}$, $\mathrm{H} 2 \alpha), 1.54(1 \mathrm{H}, \mathrm{m}, \mathrm{H} 2 \beta), 1.53\left(1 \mathrm{H}, \mathrm{d}, \mathrm{J}_{\mathrm{gem}}=3.5 \mathrm{~Hz}, \mathrm{H} 19 \alpha\right), 1.50(2 \mathrm{H}, \mathrm{m}, \mathrm{H} 6 \alpha+\mathrm{H} 15 \alpha), 1.48$ $(2 \mathrm{H}, \mathrm{m}, \mathrm{H} 7), 1.27(1 \mathrm{H}, \mathrm{m}, \mathrm{H} 1 \alpha), 1.21(3 \mathrm{H}, \mathrm{s}, \mathrm{H} 28), 1.15$ and $1.18(6 \mathrm{H}, 2 \mathrm{~d}, \mathrm{~J}=7.3 \mathrm{~Hz}$, H3'+H4'), $1.04\left(1 \mathrm{H}, \mathrm{d}, \mathrm{J}_{\text {gem }}=3.5 \mathrm{~Hz}, \mathrm{H} 19 \beta\right), 0.94(1 \mathrm{H}, \mathrm{m}, \mathrm{H} 6 \beta), 0.84(3 \mathrm{H}, \mathrm{s}, \mathrm{H} 18), 0.87$ (3H, d, $\left.\mathrm{J}_{21-20}=6.5 \mathrm{~Hz}, \mathrm{H} 21\right), 0.68(3 \mathrm{H}, \mathrm{s}, \mathrm{H} 30) .{ }^{13} \mathrm{C} \mathrm{NMR}\left(75.4 \mathrm{MHz}, \mathrm{CDCl}_{3}\right): \delta(\mathrm{ppm}) 211.6(\mathrm{C} 11)$, 176.9 (C1'), 78.2 (C16), 61.9 (C20), 56.6 (C29), 55.7 (C17), 51.4 (C12), 50.4 (C3), 47.0 (C14), 44.3 (C13), 43.3 (C4), 42.9 (C15), 42.6 (C5), 42.1 (C8), 38.1 (C10), 37.4 (C31), 35.8 (C2'), 34.3 (C9), 31.7 (C19), 28.2 (C2), 27.6 (C1), 24.5 (C7), 21.0 (C28), 19.4 and 20.2 (C3'+C4'), 18.6 (C6), 17.8 (C18), 13.8 (C30), 9.8 (C21) ; MS (ESI, m/z) : $516.4(\mathrm{M}+\mathrm{H})$; HRMS (ESI, m/z) : calcd for $\mathrm{C}_{31} \mathrm{H}_{54} \mathrm{~N}_{3} \mathrm{O}_{3}: 516.4165$, found : 516.4159 . 


\section{$N$-[20S-(dimethylamino)-4 $\alpha$-[(ethylamino)methyl]-16 $\alpha$-hydroxy-4 $\beta, 14 \alpha$-dimethyl-11-oxo- 9,19-cyclo-5 $\alpha, 9 \beta$-pregnan-3 $\beta$-yl]-isobutyramide (12b)}

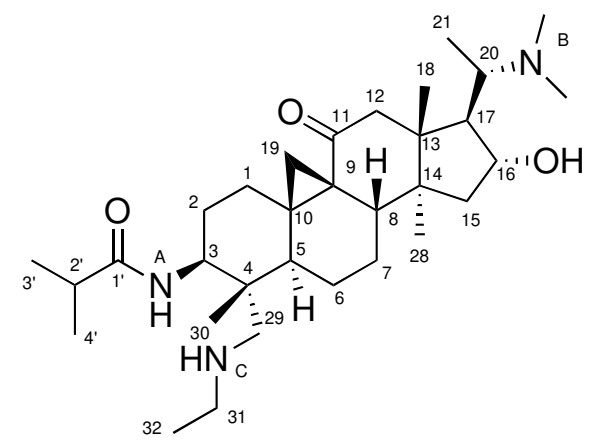

The title compound was prepared from $100 \mathrm{mg}(0.19 \mathrm{mmol}, 1$ eq. $)$ of aldehyde $\mathbf{1 0}$, $100 \mu \mathrm{L}$ (1.5 mmol, 7.5 eq.) of ethylamine, $34 \mu \mathrm{L}(0.6 \mathrm{mmol}, 3$ eq.) acetic acid, $19 \mathrm{mg}$ ( $0.3 \mathrm{mmol}, 1.5$ eq.) of sodium cyanoborohydride and $1 \mathrm{~mL}$ of EtOH by following similar procedure describe for the preparation of 12a. 12b $(90 \mathrm{mg}$, yield $85 \%)$. IR $\left(\mathrm{cm}^{-1}\right): 3308,2961$, 2933, 2869, 2831, 1732, 1651, 1537, 1453, 1226, 1188, 1165, $1096 ;{ }^{1} \mathrm{H}$ NMR $(300 \mathrm{MHz}$, $\left.\mathrm{CDCl}_{3}\right): \delta(\mathrm{ppm}) 5.46\left(1 \mathrm{H}, \mathrm{d}, \mathrm{J}_{\mathrm{NAH}-3}=9.7 \mathrm{~Hz}, \mathrm{~N}_{\mathrm{A}}-\mathrm{H}\right), 4.10(1 \mathrm{H}, \mathrm{ddd}, \mathrm{J}=10.0 \mathrm{~Hz}, \mathrm{~J}=7.3 \mathrm{~Hz}, \mathrm{~J}=$ $2.8 \mathrm{~Hz}, \mathrm{H} 16 \beta), 3.97(1 \mathrm{H}, \mathrm{ddd}, \mathrm{J}=11.9 \mathrm{~Hz}, \mathrm{~J}=9.7 \mathrm{~Hz}, \mathrm{~J}=4.2 \mathrm{~Hz}, \mathrm{H} 3 \alpha), 2.53-2.68(3 \mathrm{H}, \mathrm{m}$, $\mathrm{H} 20+\mathrm{H} 31), 2.52\left(1 \mathrm{H}, \mathrm{d}, \mathrm{J}_{\mathrm{gem}}=17.8 \mathrm{~Hz}, \mathrm{H} 12 \alpha\right), 2.41(1 \mathrm{H}, \mathrm{m}, \mathrm{H} 1 \beta), 2.36(1 \mathrm{H}, \mathrm{q}, \mathrm{J}=6.9 \mathrm{~Hz}$, H2'), $2.28\left(1 \mathrm{H}, \mathrm{d}, \mathrm{J}_{\mathrm{gem}}=17.8 \mathrm{~Hz}, \mathrm{H} 12 \beta\right), 2.25\left(8 \mathrm{H}, \mathrm{m}, \mathrm{H} 29+\mathrm{N}_{\mathrm{B}} \mathrm{Me}_{2}\right), 2.17(1 \mathrm{H}, \mathrm{m}, \mathrm{H} 5 \alpha), 2.04$ $(1 \mathrm{H}, \mathrm{m}, \mathrm{H} 15 \beta), 1.98(2 \mathrm{H}, \mathrm{m}, \mathrm{H} 17 \alpha+\mathrm{H} 8 \beta), 1.69(1 \mathrm{H}, \mathrm{m}, \mathrm{H} 2 \alpha), 1.54\left(1 \mathrm{H}, \mathrm{d}, \mathrm{J}_{\mathrm{gem}}=3.6 \mathrm{~Hz}\right.$, $\mathrm{H} 19 \alpha), 1.53(1 \mathrm{H}, \mathrm{m}, \mathrm{H} 2 \beta), 1.50(4 \mathrm{H}, \mathrm{m}, \mathrm{H} 6 \alpha+\mathrm{H} 15 \alpha+\mathrm{H} 7), 1.28(1 \mathrm{H}, \mathrm{m}, \mathrm{H} 1 \alpha), 1.22(3 \mathrm{H}, \mathrm{s}$, $\mathrm{H} 28), 1.15$ and $1.17\left(6 \mathrm{H}, 2 \mathrm{~d}, \mathrm{~J}=6.9 \mathrm{~Hz}, \mathrm{H} 3^{\prime}+\mathrm{H}^{\prime}{ }^{\prime}\right), 1.08\left(3 \mathrm{H}, \mathrm{t}, \mathrm{J}_{32-31}=7.2 \mathrm{~Hz}, \mathrm{H} 32\right), 1.04(1 \mathrm{H}$, d, $\left.\mathbf{J}_{\text {gem }}=3.6 \mathrm{~Hz}, \mathrm{H} 19 \beta\right), 0.98(1 \mathrm{H}, \mathrm{m}, \mathrm{H} 6 \beta), 0.87\left(3 \mathrm{H}, \mathrm{d}, \mathrm{J}_{21-20}=6.6 \mathrm{~Hz}, \mathrm{H} 21\right), 0.84(3 \mathrm{H}, \mathrm{s}, \mathrm{H} 18)$, 0.69 (3H, s, H30) ; ${ }^{13} \mathrm{C}$ NMR (75.4 MHz, $\left.\mathrm{CDCl}_{3}\right)$ : $\delta(\mathrm{ppm}) 211.6(\mathrm{C} 11), 176.9\left(\mathrm{Cl}{ }^{\prime}\right), 78.3(\mathrm{C} 16)$, 61.9 (C20), 55.7 (C17), 53.4 (C29), 51.4 (C12), 50.4 (C3), 47.0 (C14), 44.7 (C31), 44.3 (C13), 43.1 (C4), 42.8 (C15), 42.3 (C5), 42.0 (C8), 38.1 (C10), 35.8 (C2'), 34.3 (C9), 31.5 (C19), 28.2 (C2), 27.5 (C1), 24.4 (C7), 21.0 (C28), 19.4 and 20.1 (C3'+C4'), 18.6 (C6), 17.8 (C18), 14.7 (C32), 13.8 (C30), 9.8 (C21) ; MS (ESI, m/z) : $530.5(\mathrm{M}+\mathrm{H})$; HRMS (ESI, m/z) : calcd for $\mathrm{C}_{32} \mathrm{H}_{56} \mathrm{~N}_{3} \mathrm{O}_{3}: 530.4322$, found : 530.4299 . 


\section{$20 S$-(dimethylamino)-3 $\beta$-(isobutanamido)-4 $\alpha$-(hydroxymethyl)-4 $\beta, 14 \alpha$-dimethyl-11-oxo-}

9,19-cyclo-5 $\alpha, 9 \beta$-pregnan-16 $\alpha$-yl benzoate (14)

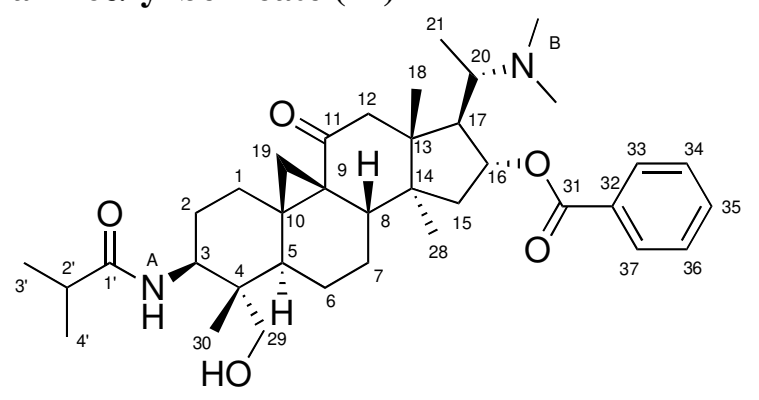

To $216 \mathrm{mg}$ ( $0.429 \mathrm{mmol}, 1$ eq.) of $N$-3-isobutyrylcycloxobuxidine-F 3 solubilized in $9 \mathrm{~mL}$ of a mixture DCM/pyridine (3/1) were added at $0{ }^{\circ} \mathrm{C} 130 \mathrm{mg}(0.515 \mathrm{mmol}, 1.2$ eq. $)$ of benzoic anhydride. The mixture was stirred at $0{ }^{\circ} \mathrm{C}$ for $1 \mathrm{~h}$ and at RT for further $12 \mathrm{~h}$. After 3 coevaporations with $3 \times 10 \mathrm{~mL}$ of 1,2-dichloroethane, the residue was dissolved in DCM, washed with $20 \mathrm{~mL}$ of a saturated sodium hydrogen carbonate solution and extracted with $3 \times 20 \mathrm{~mL}$ of DCM. Combined organic layers were washed with brine, dried over magnesium sulfate and concentrated under vacuum. The crude product was purified by silica gel column chromatography (eluent : $\mathrm{DCM} / \mathrm{MeOH} / \mathrm{NH}_{4} \mathrm{OH}: 97 / 2 / 1$ ) to afford the title compound as a white solid (260 mg, yield $100 \%$ ). IR $\left(\mathrm{cm}^{-1}\right): 3314,2965,2930,2873,2829,1702,1661,1620,1540$, 1447, 1279, 1230, $1114 ;{ }^{1} \mathrm{H}$ NMR (300 MHz, CDCl $): \delta(\mathrm{ppm}) 8.04(2 \mathrm{H}, \mathrm{m}, \mathrm{H} 33+\mathrm{H} 37), 7.54$ $(1 \mathrm{H}, \mathrm{m}, \mathrm{H} 35), 7.43(2 \mathrm{H}, \mathrm{m}, \mathrm{H} 34+\mathrm{H} 36), 5.41\left(2 \mathrm{H}, \mathrm{m}, \mathrm{N}_{\mathrm{A}}-\mathrm{H}+\mathrm{H} 16 \beta\right), 4.35\left(1 \mathrm{H}, \mathrm{dd}, \mathrm{J}_{\mathrm{OH}-\mathrm{H} 29 \mathrm{~b}}=\right.$ $\left.10.9 \mathrm{~Hz}, \mathrm{~J}_{\mathrm{OH}-\mathrm{H} 29 \mathrm{a}}=4.9 \mathrm{~Hz}, \mathrm{OH} 29\right), 3.99(1 \mathrm{H}, \mathrm{m}, \mathrm{H} 3 \alpha), 3.33\left(1 \mathrm{H}, \mathrm{dd}, \mathrm{J}_{\mathrm{gem}}=12.7 \mathrm{~Hz}, \mathrm{~J}_{\mathrm{H} 29 \mathrm{~b}-\mathrm{OH}}=\right.$ $10.9 \mathrm{~Hz}, \mathrm{H} 29 \mathrm{~b}), 2.96\left(1 \mathrm{H}, \mathrm{dd}, \mathrm{J}_{\mathrm{gem}}=12.7 \mathrm{~Hz}, \mathrm{~J}_{\mathrm{H} 29 \mathrm{a}-\mathrm{OH}}=4.9 \mathrm{~Hz}, \mathrm{H} 29 \mathrm{a}\right), 2.62\left(1 \mathrm{H}, \mathrm{d}, \mathrm{J}_{\mathrm{gem}}=\right.$ $17.1 \mathrm{~Hz}, \mathrm{H} 12 \alpha), 2.50\left(1 \mathrm{H}, \mathrm{dq}, \mathrm{J}_{20-17 \alpha}=11.3 \mathrm{~Hz}, \mathrm{~J}_{20-21}=6.6 \mathrm{~Hz}, \mathrm{H} 20\right), 2.44(2 \mathrm{H}, \mathrm{m}, \mathrm{H} 1 \beta+\mathrm{H} 17 \alpha)$, $2.43\left(1 \mathrm{H}, \quad \mathrm{d}, \quad \mathrm{J}_{\mathrm{gem}}=17.1 \mathrm{~Hz}, \mathrm{H} 12 \beta\right), 2.40 \quad\left(1 \mathrm{H}, \quad \mathrm{m}, \quad \mathrm{H} 2^{\prime}\right), \quad 2.02-2.17 \quad(9 \mathrm{H}, \mathrm{m}$, $\left.\mathrm{N}_{\mathrm{B}} \mathrm{Me}_{2}+\mathrm{H} 5 \alpha+\mathrm{H} 8 \beta+\mathrm{H} 15 \beta\right), 1.68(2 \mathrm{H}, \mathrm{m}, \mathrm{H} 2 \alpha+\mathrm{H} 2 \beta), 1.68(1 \mathrm{H}, \mathrm{m}, \mathrm{H} 6 \alpha), 1.55-1.60(2 \mathrm{H}, \mathrm{m}$, $\mathrm{H} 19 \alpha+\mathrm{H} 15 \alpha), 1.47(1 \mathrm{H}, \mathrm{m}, \mathrm{H} 7 \beta), 1.38(1 \mathrm{H}, \mathrm{m}, \mathrm{H} 7 \alpha), 1.27(1 \mathrm{H}, \mathrm{m}, \mathrm{H} 1 \alpha), 1.24(3 \mathrm{H}, \mathrm{s}, \mathrm{H} 28)$, 1.17 and $1.19\left(6 \mathrm{H}, 2 \mathrm{~d}, \mathrm{~J}=6.9 \mathrm{~Hz}, \mathrm{H} 3^{\prime}+\mathrm{H} 4{ }^{\prime}\right), 1.06\left(1 \mathrm{H}, \mathrm{d}, \mathrm{J}_{\mathrm{gem}}=3.5 \mathrm{~Hz}, \mathrm{H} 19 \beta\right), 0.89(1 \mathrm{H}, \mathrm{m}$, $\mathrm{H} 6 \beta), 0.91(3 \mathrm{H}, \mathrm{s}, \mathrm{H} 18), 0.85\left(3 \mathrm{H}, \mathrm{d}, \mathrm{J}_{21-20}=6.1 \mathrm{~Hz}, \mathrm{H} 21\right), 0.57(3 \mathrm{H}, \mathrm{s}, \mathrm{H} 30) ;{ }^{13} \mathrm{C}$ NMR $(75.4$ $\mathrm{MHz}, \mathrm{CDCl}_{3}$ ): $\delta(\mathrm{ppm}) 211.2$ (C11), 178.5 (C1'), 165.9 (C31), 132.5 (C35), 130.9 (C32), 129.4 (C33+C37), 128.4 (C34+C36), 79.5 (C16), 64.1 (C29), 59.5 (C20), 55.4 (C17), 52.0 (C12), 50.7 (C3), 47.6 (C14), 44.7 (C13), 44.6 (C4), 43.2 (C15), 41.5 (C8), 41.2 (C5), $40.3\left(\mathrm{~N}_{\mathrm{B}} \mathrm{Me}_{2}\right), 37.8$ (C10), 35.8 (C2'), 34.0 (C9), 30.8 (C19), 27.8 (C1), 27.6 (C2), 24.4 (C7), 20.1 (C28), 19.6 and 19.4 (C3'+C4'), 18.4 (C6), 17.9 (C18), 11.2 (C30), 9.9 (C21) ; MS (ESI, m/z) : $607.3(\mathrm{M}+\mathrm{H})$; HRMS (ESI, m/z) : calcd for $\mathrm{C}_{37} \mathrm{H}_{55} \mathrm{~N}_{2} \mathrm{O}_{5}: 607.4070$, found : 607.4111 . 


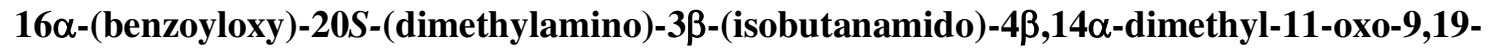
cyclo-5 $\alpha, 9 \beta$-pregnan-4 $\alpha$-carboxylic acid (15)

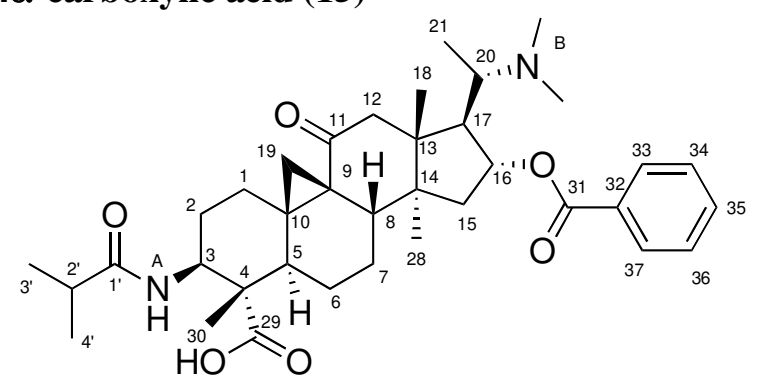

To $300 \mathrm{mg}$ ( $0.494 \mathrm{mmol}, 1 \mathrm{eq}$.) of $\mathbf{1 4}$ solubilized in $15 \mathrm{~mL}$ of acetone were added at $0{ }^{\circ} \mathrm{C} 925 \mu \mathrm{L}$ (2.47 mmol, 5 eq.) of Jones' reagent $(2.672 \mathrm{M})$. The mixture was stirred at RT for $1 \mathrm{~h}$ then cooled at $0^{\circ} \mathrm{C} .5 \mathrm{~mL}$ of isopropanol were slowly added to quench the excess of reagent. $5 \mathrm{~mL}$ of $\mathrm{NH}_{4} \mathrm{OH}$ were added and the mixture was filtered through a pad of celite. The filtrate was concentrated under vacuum and the residue was purified by silica gel column chromatography (eluent : $\mathrm{DCM} / \mathrm{MeOH} / \mathrm{NH}_{4} \mathrm{OH}: 85 / 15 / 1$ ) to afford the title compound as a white solide (224 mg, yield $73 \%$ ). IR $\left(\mathrm{cm}^{-1}\right): 3354,2966,2930,2873,2829,1711,1660,1650$, $1555,1449,1279,1228,1112 ;{ }^{1} \mathrm{H}$ NMR $\left(300 \mathrm{MHz}, \mathrm{CD}_{3} \mathrm{OD}\right): \delta(\mathrm{ppm}) 8.03(2 \mathrm{H}, \mathrm{m}, \mathrm{H} 33+\mathrm{H} 37)$, $7.57(1 \mathrm{H}, \mathrm{m}, \mathrm{H} 35), 7.45(2 \mathrm{H}, \mathrm{m}, \mathrm{H} 34+\mathrm{H} 36), 5.43(1 \mathrm{H}, \mathrm{m}, \mathrm{H} 16 \beta), 4.32(1 \mathrm{H}, \mathrm{m}, \mathrm{H} 3 \alpha), 2.93(1 \mathrm{H}$, $\left.\mathrm{dq}, \mathrm{J}_{20-17 \alpha}=11.0 \mathrm{~Hz}, \mathrm{~J}_{20-21}=6.4 \mathrm{~Hz}, \mathrm{H} 20\right), 2.74\left(1 \mathrm{H}, \mathrm{d}, \mathrm{J}_{\mathrm{gem}}=18.4 \mathrm{~Hz}, \mathrm{H} 12 \alpha\right), 2.67(1 \mathrm{H}, \mathrm{m}$,

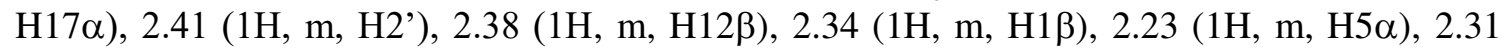
$\left(6 \mathrm{H}, \mathrm{s}, \mathrm{N}_{\mathrm{B}} \mathrm{Me}_{2}\right), 2.18(1 \mathrm{H}, \mathrm{m}, \mathrm{H} 8 \beta), 1.73(2 \mathrm{H}, \mathrm{m}, \mathrm{H} 2 \alpha+\mathrm{H} 2 \beta), 1.59$ (2H, m, H15 $\left.\alpha+\mathrm{H} 15 \beta\right), 1.56$ $(1 \mathrm{H}, \mathrm{m}, \mathrm{H} 19 \alpha), 1.48(2 \mathrm{H}, \mathrm{m}, \mathrm{H} 7 \beta+\mathrm{H} 7 \alpha), 1.33(1 \mathrm{H}, \mathrm{m}, \mathrm{H} 1 \alpha), 1.29(3 \mathrm{H}, \mathrm{s}, \mathrm{H} 28), 1.20(1 \mathrm{H}, \mathrm{m}$,

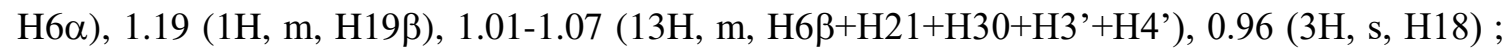
${ }^{13} \mathrm{C}$ NMR (75.4 MHz, CD $\left.{ }_{3} \mathrm{OD}\right): \delta(\mathrm{ppm}) 212.9(\mathrm{C} 11), 181.3(\mathrm{C} 29), 179.2(\mathrm{C} 1$ '), $167.4(\mathrm{C} 31)$, 134.1 (C35), 132.0 (C32), 130.4 (C33+C37), 129.6 (C34+C36), 80.9 (C16), 61.8 (C20), 55.9 (C17), 55.3 (C3), 54.5 (C4), 53.0 (C12), 48.4 (C14), 46.5 (C5), 46.4 (C13), 44.3 (C15), 42.5 (C8), $40.6\left(\mathrm{~N}_{\mathrm{B}} \mathrm{Me}_{2}\right), 39.2$ (C10), 36.1 (C2'), 35.2 (C9), 31.5 (C19), 28.9 (C1), 28.7 (C2), 25.5 (C7), 22.1 (C6), 20.1 and 20.2 (C3'+C4'), 19.9 (C28), 18.3 (C18), 11.4 (C30), 10.7 (C21); MS $\left(\right.$ ESI, m/z) : $621.3(\mathrm{M}+\mathrm{H})$; HRMS $(\mathrm{ESI}, \mathrm{m} / \mathrm{z})$ : calcd for $\mathrm{C}_{37} \mathrm{H}_{53} \mathrm{~N}_{2} \mathrm{O}_{6}: 621.3904$, found : 621.3896. 


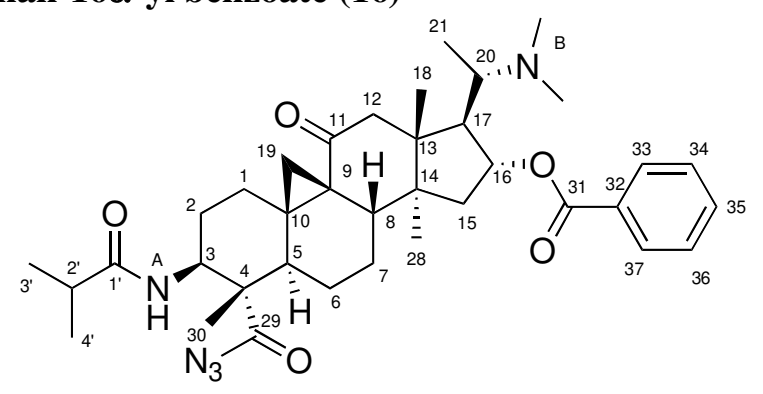

To $350 \mathrm{mg}$ ( $0.564 \mathrm{mmol}, 1$ eq.) of $\mathbf{1 5}$ solubilized in $7 \mathrm{~mL}$ of DCM were added at $-20{ }^{\circ} \mathrm{C}$ $235 \mu \mathrm{L}$ (1.69 mmol, 3 eq.) of TEA, $44 \mu \mathrm{L}(0.564 \mathrm{mmol}, 1$ eq.) of DMF and $74 \mu \mathrm{L}(0.845 \mathrm{mmol}$, 1.5 eq.) of oxalyl chloride. The mixture was stirred at $-20^{\circ} \mathrm{C}$ for 15 min then warmed to $0{ }^{\circ} \mathrm{C}$. $183 \mathrm{mg}$ ( $2.8 \mathrm{mmol}, 5$ eq.) of sodium azide dissolved in the minimum of water were added. The mixture was stirred at RT for further $3 \mathrm{~h}$, washed with $20 \mathrm{~mL}$ of a saturated sodium hydrogen carbonate solution and extracted with $3 \times 20 \mathrm{~mL}$ of DCM. Combined organic layers were washed with brine, dried over magnesium sulfate and concentrated under vacuum. The crude product was purified by silica gel column chromatography (eluent : ACOEt/heptane/ $\mathrm{NH}_{4} \mathrm{OH}: 50 / 50 / 1$ ) to afford the title compound as a white solid (350 mg, yield: $74 \%)$. IR $\left(\mathrm{cm}^{-1}\right): 3316,2933$, 2870, $2137\left(\mathrm{vCO}^{-\mathrm{N}_{3}}\right), 1744,1708,1665,1530,1449,1194,1218,1276 ;{ }^{1} \mathrm{H}$ NMR $(300 \mathrm{MHz}$, $\left.\mathrm{CDCl}_{3}\right): \delta(\mathrm{ppm}) 8.05(2 \mathrm{H}, \mathrm{m}, \mathrm{H} 33+\mathrm{H} 37), 7.56(1 \mathrm{H}, \mathrm{m}, \mathrm{H} 35), 7.44(2 \mathrm{H}, \mathrm{m}, \mathrm{H} 34+\mathrm{H} 36), 5.41(1 \mathrm{H}$, $\mathrm{m}, \mathrm{H} 16 \beta), 5.36\left(1 \mathrm{H}, \mathrm{d}, \mathrm{J}_{\mathrm{NAH}-3}=9.5 \mathrm{~Hz}, \mathrm{~N}_{\mathrm{A}} \mathrm{H}\right), 4.38(1 \mathrm{H}, \mathrm{m}, \mathrm{H} 3 \alpha), 2.63\left(1 \mathrm{H}, \mathrm{d}, \mathrm{J}_{\text {gem }}=17.0 \mathrm{~Hz}\right.$, $\mathrm{H} 12 \alpha), 2.50$ (1H, m, H20), 2.45 (2H, m, H17 $\alpha+\mathrm{H} 12 \beta), 2.36$ (1H, m, H1 $\beta$ ), 2.29 (1H, m, H2'), $2.25(1 \mathrm{H}, \mathrm{m}, \mathrm{H} 5 \alpha), 2.09$ (6H, s, $\left.\mathrm{N}_{\mathrm{B}} \mathrm{Me}_{2}\right), 2.12$ (1H, m, H8 $\beta$ ), $1.80(2 \mathrm{H}, \mathrm{m}, \mathrm{H} 2 \alpha+\mathrm{H} 2 \beta), 1.54-1.63$ $(3 \mathrm{H}, \mathrm{m}, \mathrm{H} 15 \alpha+\mathrm{H} 15 \beta+\mathrm{H} 19 \alpha), 1.48$ (2H, m, H7 $\beta), 1.40$ (2H, m, H7 $\alpha+\mathrm{H} 1 \alpha), 1.28$ (3H, s, H28), 1.05-1.11 (9H, m, H6+H19ß+H3'+H4'), 1.07 (3H, s, H30), $0.90(3 \mathrm{H}, \mathrm{s}, \mathrm{H} 18), 0.85$ (3H, d, $\left.\mathrm{J}_{21-20}=5.9 \mathrm{~Hz}, \mathrm{H} 21\right) ;{ }^{13} \mathrm{C} \mathrm{NMR}\left(75.4 \mathrm{MHz}, \mathrm{CDCl}_{3}\right): \delta(\mathrm{ppm}) 210.4(\mathrm{C} 11), 183.4(\mathrm{C} 29), 176.2$ (C1'), 165.7 (C31), 132.6 (C35), 130.7 (C32), 129.3 (C33+C37), 128.2 (C34+C36), 79.4 (C16), 59.4 (C20), 55.4 (C17), 54.7 (C4), 52.7 (C3), 51.8 (C12), 47.5 (C14), 44.6 (C13), 44.1 (C5), 42.9 (C15), 40.9 (C8), $40.2\left(\mathrm{~N}_{\mathrm{B}} \mathrm{Me}_{2}\right), 36.2$ (C10), 35.7 (C2'), 33.7 (C9), 29.4 (C19), 27.9 (C2), 27.2 (C1), 23.9 (C7), 21.1 (C6), 19.9 (C28), 19.3 and 19.4 (C3'+C4'), 17.9 (C18), 10.0 (C30), $9.9(\mathrm{C} 21)$; MS (ESI, m/z) : $646.4(\mathrm{M}+\mathrm{H}), 618.3$; HRMS (ESI, m/z) : calcd for $\mathrm{C}_{37} \mathrm{H}_{52} \mathrm{~N}_{5} \mathrm{O}_{5}$ : 646.3968, found : 646.3952 . 


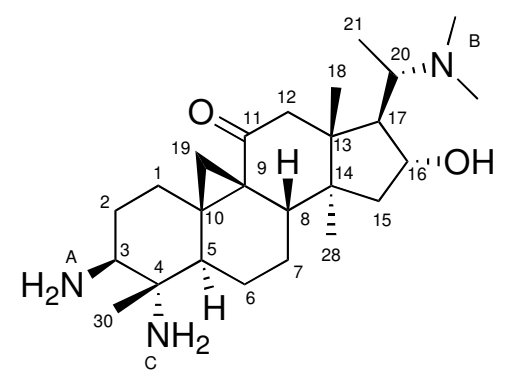

$155 \mathrm{mg}(0.24 \mathrm{mmol}, 1$ eq.) of $\mathbf{1 6}$ were heated without solvent in a microwave (Pmax = $200 \mathrm{~W}$ ) at $110{ }^{\circ} \mathrm{C}$ for $10 \mathrm{~min} .20 \mathrm{~mL}$ of THF, $5 \mathrm{~mL}$ of water and a small amount of lithium hydroxide were added and the mixture was refluxed for $12 \mathrm{~h} .5 \mathrm{~mL}$ of $\mathrm{MeOH}$ were added and the mixture was refluxed for further $1 \mathrm{~h}$. Solvents were removed under vacuum and $50 \mathrm{~mL}$ of $\mathrm{HCl}(2 \mathrm{~N})$ were added to the residue. The mixture was refluxed for $1 \mathrm{~h}$ and concentrated under vacuum to leave $5 \mathrm{~mL}$ of aqueous layer. This layer was alkalinised with $\mathrm{NH}_{4} \mathrm{OH}$ and extracted with $5 \times 20 \mathrm{~mL}$ of DCM. Combined organic layers were washed with brine, dried over magnesium sulfate and concentrated under vacuum. The crude product was purified by silica gel column chromatography (eluent : $\mathrm{DCM} / \mathrm{MeOH} / \mathrm{NH}_{4} \mathrm{OH}: 80 / 20 / 1$ ) to afford $76 \mathrm{mg}$ of the title compound and $10 \mathrm{mg}$ of $\mathbf{1 9}$ (respective ratio: 9/1) as amorphous white solids (overall yield: $84 \%)$. IR $\left(\mathrm{cm}^{-1}\right): 3210(\mathrm{vN}-\mathrm{H}, \mathrm{vO}-\mathrm{H}), 2966-2941,2873,1702,1667,1459,1250,1222 ;{ }^{1} \mathrm{H}$ NMR $\left(300 \mathrm{MHz}, \mathrm{CDCl}_{3}\right): \delta(\mathrm{ppm}) 4.10(1 \mathrm{H}, \mathrm{ddd}, \mathrm{J}=10.1 \mathrm{~Hz}, \mathrm{~J}=7.3 \mathrm{~Hz}, \mathrm{~J}=2.9 \mathrm{~Hz}, \mathrm{H} 16 \beta)$, $2.62\left(1 \mathrm{H}, \mathrm{dq}, \mathrm{J}_{20-17 \alpha}=10.9 \mathrm{~Hz}, \mathrm{~J}_{20-21}=6.5 \mathrm{~Hz}, \mathrm{H} 20\right), 2.50\left(1 \mathrm{H}, \mathrm{d}, \mathrm{J}_{\mathrm{gem}}=17.1 \mathrm{~Hz}, \mathrm{H} 12 \alpha\right), 2.43$ $(1 \mathrm{H}, \mathrm{dd}, \mathrm{J}=10.4 \mathrm{~Hz}, \mathrm{~J}=4.1 \mathrm{~Hz}, \mathrm{H} 3 \alpha), 2.30\left(1 \mathrm{H}, \mathrm{d}, \mathrm{J}_{\mathrm{gem}}=17.1 \mathrm{~Hz}, \mathrm{H} 12 \beta\right), 2.30(1 \mathrm{H}, \mathrm{m}, \mathrm{H} 1 \beta)$, $2.24\left(6 \mathrm{H}, \mathrm{bs}, \mathrm{N}_{\mathrm{B}} \mathrm{Me}_{2}\right), 2.07(1 \mathrm{H}, \mathrm{m}, \mathrm{H} 8 \beta), 2.02(1 \mathrm{H}, \mathrm{m}, \mathrm{H} 15 \beta), 1.97(1 \mathrm{H}, \mathrm{m}, \mathrm{H} 17 \alpha), 1.76(2 \mathrm{H}$, $\mathrm{m}, \mathrm{H} 2+\mathrm{C} 6 \beta), 1.60(1 \mathrm{H}, \mathrm{m}, \mathrm{H} 7 \alpha), 1.54\left(1 \mathrm{H}, \mathrm{d}, \mathrm{J}_{\mathrm{gem}}=4.0 \mathrm{~Hz}, \mathrm{H} 19 \alpha\right), 1.49(1 \mathrm{H}, \mathrm{m}, \mathrm{H} 15 \alpha), 1.46$ $(1 \mathrm{H}, \mathrm{m}, \mathrm{H} 5 \alpha), 1.36(1 \mathrm{H}, \mathrm{m}, \mathrm{H} 7 \beta), 1.32(1 \mathrm{H}, \mathrm{m}, \mathrm{H} 2 \beta), 1.25(1 \mathrm{H}, \mathrm{m}, \mathrm{H} 1 \alpha), 1.21(3 \mathrm{H}, \mathrm{s}, \mathrm{H} 28)$, $1.02(1 \mathrm{H}, \mathrm{m}, \mathrm{H} 6 \alpha), 1.05\left(1 \mathrm{H}, \mathrm{d}, \mathrm{J}_{\mathrm{gem}}=4.0 \mathrm{~Hz}, \mathrm{H} 19 \beta\right), 0.87\left(3 \mathrm{H}, \mathrm{d}, \mathrm{J}_{21-20}=6.6 \mathrm{~Hz}, \mathrm{H} 21\right), 0.85$ (3H, s, H30), $0.84(3 \mathrm{H}, \mathrm{s}, \mathrm{H} 18) ;{ }^{13} \mathrm{C}$ NMR (75.4 MHz, $\left.\mathrm{CDCl}_{3}\right): \delta(\mathrm{ppm}) 211.0(\mathrm{C} 11), 78.3(\mathrm{C} 16)$, 62.0 (C20), 60.2 (C3), 57.0 (C4), 55.7 (C17), 51.4 (C12), 50.7 (C5), 47.1 (C14), 44.5 (C13), 42.7 (C15), 41.2 (C8), 39.0 (C10), 35.0 (C9), 31.6 (C2), 29.7 (C19), 27.7 (C1), 24.4 (C7), 20.6 (C28), 18.4 (C6), 17.8 (C18), 15.2 (C30), 9.9 (C21) ; MS (ESI, m/z) : 418.3 (M+H) ; HRMS (ESI, m/z) : calcd for $\mathrm{C}_{25} \mathrm{H}_{43} \mathrm{~N}_{3} \mathrm{O}_{2}: 418.3433$, found : 418.3450 . 


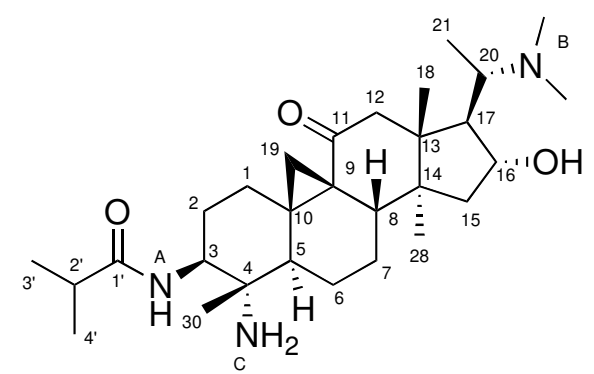

$\operatorname{MP}\left({ }^{\circ} \mathrm{C}\right): 254$; IR $\left(\mathrm{cm}^{-1}\right): 3314,2970,2932,2873,2832,1652,1540,1456,1226,1189,1166$; ${ }^{1} \mathrm{H}$ NMR $\left(300 \mathrm{MHz}, \mathrm{CDCl}_{3}\right): \delta(\mathrm{ppm}) 5.36\left(1 \mathrm{H}, \mathrm{d}, \mathrm{J}_{\mathrm{NAH}-3}=9.1 \mathrm{~Hz}, \mathrm{~N}_{\mathrm{A}} \mathrm{H}\right), 4.10(1 \mathrm{H}, \mathrm{m}, \mathrm{H} 16 \beta)$, $3.78(1 \mathrm{H}, \mathrm{m}, \mathrm{H} 3 \alpha), 2.62\left(1 \mathrm{H}, \mathrm{dq}, \mathrm{J}_{20-17 \alpha}=10.9 \mathrm{~Hz}, \mathrm{~J}_{20-21}=6.6 \mathrm{~Hz}, \mathrm{H} 20\right), 2.52\left(1 \mathrm{H}, \mathrm{d}, \mathrm{J}_{\mathrm{gem}}=\right.$ $17.0 \mathrm{~Hz}, \mathrm{H} 12 \alpha), 2.39\left(1 \mathrm{H}, \mathrm{m}, \mathrm{H} 2^{\prime}\right), 2.34(1 \mathrm{H}, \mathrm{m}, \mathrm{H} 1 \beta), 2.30(1 \mathrm{H}, \mathrm{m}, \mathrm{H} 12 \beta), 2.25(6 \mathrm{H}, \mathrm{s}$,

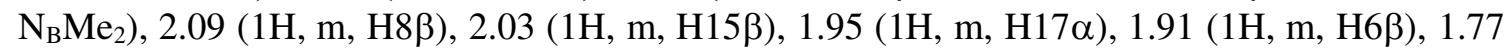
$(2 \mathrm{H}, \mathrm{m}, \mathrm{H} 2 \alpha+\mathrm{H} 2 \beta), 1.62(2 \mathrm{H}, \mathrm{m}, \mathrm{H} 5 \alpha+\mathrm{H} 7 \beta), 1.53(1 \mathrm{H}, \mathrm{d}, \mathrm{J}$ gem $=4.0 \mathrm{~Hz}, \mathrm{H} 19 \alpha), 1.50(1 \mathrm{H}, \mathrm{m}$, $\mathrm{H} 15 \alpha), 1.38(1 \mathrm{H}, \mathrm{m}, \mathrm{H} 7 \alpha), 1.33(1 \mathrm{H}, \mathrm{m}, \mathrm{H} 1 \alpha), 1.22(3 \mathrm{H}, \mathrm{s}, \mathrm{H} 28), 1.17$ and $1.18(6 \mathrm{H}, 2 \mathrm{~d}, \mathrm{~J}=$ $6.9 \mathrm{~Hz}, \mathrm{H} 3{ }^{\prime}+\mathrm{H} 4$ '), $1.05\left(1 \mathrm{H}, \mathrm{d}, \mathrm{J}_{\text {gem }}=6.9 \mathrm{~Hz}, \mathrm{H} 19 \beta\right), 0.95(1 \mathrm{H}, \mathrm{m}, \mathrm{H} 6 \alpha), 0.89\left(3 \mathrm{H}, \mathrm{d}, \mathrm{J}_{21-20}=\right.$ $6.6 \mathrm{~Hz}, \mathrm{H} 21), 0.86(3 \mathrm{H}, \mathrm{s}, \mathrm{H} 30), 0.84(3 \mathrm{H}, \mathrm{s}, \mathrm{H} 18) ;{ }^{13} \mathrm{C} \mathrm{NMR}\left(75.4 \mathrm{MHz}, \mathrm{CDCl}_{3}\right): \delta(\mathrm{ppm})$ 211.1 (C11), 177.3 (C1'), 78.2 (C16), 62.0 (C20), 58.3 (C3), 56.7 (C4), 55.8 (C17), 51.4 (C12), 50.0 (C5), 47.1 (C14), 44.5 (C13), 42.7 (C15), 41.1 (C8), 38.3 (C10), 36.0 (C2'), 35.0 (C9), 29.4 (C19), 29.0 (C2), 27.5 (C1), 24.2 (C7), 20.6 (C28), 19.6 and 19.9 (C3'+C4'), 18.2 (C6), 17.8 (C18), 16.2 (C30), 9.9 (C21) ; MS (ESI, m/z) : $488.4(\mathrm{M}+\mathrm{H})$; HRMS (ESI, m/z) : calcd for $\mathrm{C}_{29} \mathrm{H}_{50} \mathrm{~N}_{3} \mathrm{O}_{3}: 488.3852$, found : 488.3848 . 


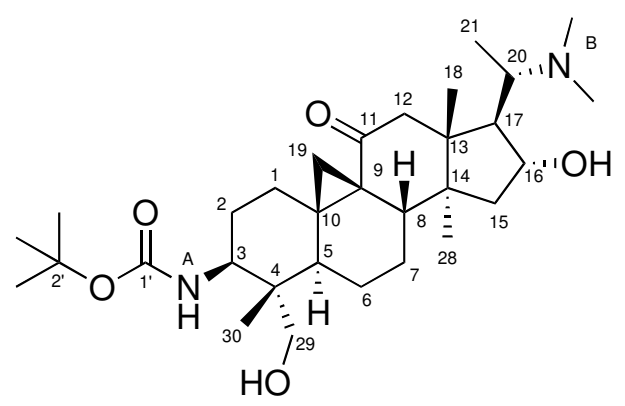

To $788 \mathrm{mg}$ ( $1.82 \mathrm{mmol}, 1$ eq.) of cycloxobuxidine-F 4 dissolved in $25 \mathrm{~mL}$ of $\mathrm{MeOH}$ were added $429 \mu \mathrm{L}$ ( $2.0 \mathrm{mmol}, 1.1$ eq.) of di-tert-butyl dicarbonate. The mixture was stirred at $\mathrm{RT}$ for $5 \mathrm{~h}$ and the solvent was removed under reduced pressure. The residue was dissolved in DCM, washed with $30 \mathrm{~mL}$ of a saturated sodium carbonate solution and extracted with $3 \times 30 \mathrm{~mL}$ of DCM. Combined organic layers were washed with brine, dried over magnesium sulfate. The solvent was removed under reduced pressure to afford the title compound as an amorphous white solid. (970 mg, yield: $100 \%$ ). IR $\left(\mathrm{cm}^{-1}\right)$ : 3313, 2971, 2935, 2870, 1667, 1530, 1454, 1251, 1164 ; ${ }^{1} \mathrm{H}$ NMR $\left(300 \mathrm{MHz}, \mathrm{CDCl}_{3}\right): \delta(\mathrm{ppm}) 4.64\left(1 \mathrm{H}, \mathrm{d}, \mathrm{J}=9.2 \mathrm{~Hz}, \mathrm{~N}_{\mathrm{A}}-\mathrm{H}\right), 4.09(2 \mathrm{H}, \mathrm{m}$, $\mathrm{H} 16 \beta+\mathrm{OH} 29), 3.69(1 \mathrm{H}, \mathrm{ddd}, \mathrm{J}=12.4 \mathrm{~Hz}, \mathrm{~J}=9.2 \mathrm{~Hz}, \mathrm{~J}=4.1 \mathrm{~Hz}, \mathrm{H} 3 \alpha), 3.35\left(1 \mathrm{H}, \mathrm{dd}, \mathrm{J}_{\mathrm{gem}}=\right.$ $\left.12.7 \mathrm{~Hz}, \mathrm{~J}_{\mathrm{H} 29 \mathrm{~b}-\mathrm{OH}}=10.3 \mathrm{~Hz}, \mathrm{H} 29 \mathrm{~b}\right), 3.13\left(1 \mathrm{H}, \mathrm{dd}, \mathrm{J}_{\mathrm{gem}}=12.7 \mathrm{~Hz}, \mathrm{~J}_{\mathrm{H} 29 \mathrm{a}-\mathrm{OH}}=4.4 \mathrm{~Hz}, \mathrm{H} 29 \mathrm{a}\right), 2.61$ $\left(1 \mathrm{H}, \mathrm{dq}, \mathrm{J}_{20-17 \alpha}=10.7 \mathrm{~Hz}, \mathrm{~J}_{20-21}=6.6 \mathrm{~Hz}, \mathrm{H} 20\right), 2.51\left(1 \mathrm{H}, \mathrm{d}, \mathrm{J}_{\mathrm{gem}}=17.1 \mathrm{~Hz}, \mathrm{H} 12 \alpha\right), 2.39(1 \mathrm{H}$, $\mathrm{dm}, \mathrm{J}=13.3 \mathrm{~Hz}, \mathrm{H} 1 \beta), 2.28\left(1 \mathrm{H}, \mathrm{d}, \mathrm{J}_{\mathrm{gem}}=17.1 \mathrm{~Hz}, \mathrm{H} 12 \beta\right), 2.25\left(6 \mathrm{H}, \mathrm{bs}, \mathrm{N}_{\mathrm{B}} \mathrm{Me}_{2}\right), 2.02$ $(\mathrm{H} 5 \alpha+\mathrm{H} 8 \beta+\mathrm{H} 15 \beta), 1.97(1 \mathrm{H}, \mathrm{m}, \mathrm{H} 17 \alpha), 1.72(1 \mathrm{H}, \mathrm{m}, \mathrm{H} 2 \alpha), 1.69(1 \mathrm{H}, \mathrm{m}, \mathrm{H} 6 \alpha), 1.55(1 \mathrm{H}, \mathrm{d}$, $\mathrm{J}=3.9 \mathrm{~Hz}, \mathrm{H} 19 \alpha), 1.52(3 \mathrm{H}, \mathrm{m}, \mathrm{H} 2 \beta+\mathrm{H} 7), 1.48(1 \mathrm{H}, \mathrm{m}, \mathrm{H} 15 \alpha), 1.45\left(9 \mathrm{H}, \mathrm{s}, \mathrm{Me}_{3}\right), 1.29(1 \mathrm{H}, \mathrm{m}$, $\mathrm{H} 1 \alpha), 1.22(3 \mathrm{H}, \mathrm{s}, \mathrm{H} 28), 1.04\left(1 \mathrm{H}, \mathrm{d}, \mathrm{J}_{\mathrm{gem}}=3.9 \mathrm{~Hz}, \mathrm{H} 19 \beta\right), 0.90(1 \mathrm{H}, \mathrm{m}, \mathrm{H} 6 \beta), 0.87(3 \mathrm{H}, \mathrm{d}$, $\left.\mathrm{J}_{21-20}=6.6 \mathrm{~Hz}, \mathrm{H} 21\right), 0.84(3 \mathrm{H}, \mathrm{s}, \mathrm{H} 18), 0.56(3 \mathrm{H}, \mathrm{s}, \mathrm{H} 30) ;{ }^{13} \mathrm{C} \mathrm{NMR}\left(75.4 \mathrm{MHz}, \mathrm{CDCl}_{3}\right)$ : $\delta(\mathrm{ppm}) 211.2$ (C11), 157.1 (C1'), 79.9 (C2'), 78.2 (C16), 64.1 (C29), 61.9 (C20), 55.7 (C17), 51.9 (C3), 51.4 (C12), 47.0 (C14), 44.6 (C4), 44.4 (C13), 42.7 (C15), 41.3+41.0 (C5+C8), 37.7 (C10), 34.0 (C9), 30.5 (C19), 28.2 ( $\mathrm{Me}_{3}$ ), 27.7+27.6 (C1+C2), 24.2 (C7), 20.7 (C28), 18.3 (C6), 17.7 (C18), 10.9 (C30), 9.8 (C21) ; MS (ESI, m/z) : $533.3(\mathrm{M}+\mathrm{H})$; HRMS (ESI, m/z) : calcd for $\mathrm{C}_{31} \mathrm{H}_{53} \mathrm{~N}_{2} \mathrm{O}_{5}: 533.3954$, found : 533.3965 . 


\section{$3 \beta-[($ tert-butoxycarbonyl)amino]-20S-(dimethylamino)-4 $\alpha$-(hydroxymethyl)- $4 \beta, 14 \alpha-$ dimethyl-11-oxo-9,19-cyclo-5 $\alpha, 9 \beta$-pregnan-16 $\alpha$-yl benzoate (22)}

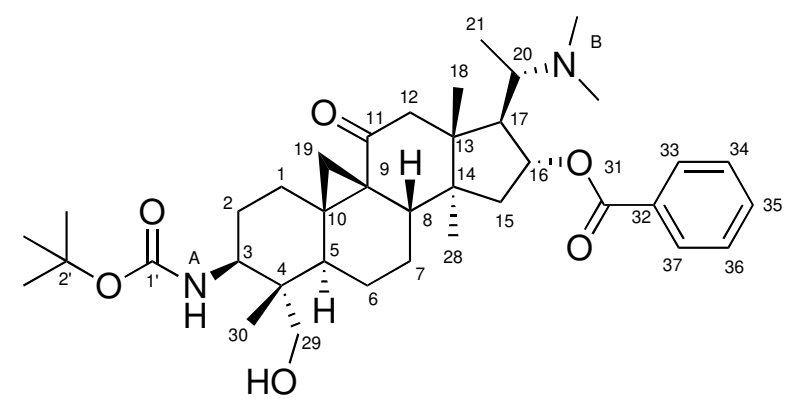

To $970 \mathrm{mg}$ (1.82 mmol, 1 eq.) of $\mathbf{2 1}$ dissolved in $25 \mathrm{~mL}$ of DCM were added $481 \mathrm{mg}$ (1.9 mmol, 1.05 eq.) of benzoic anhydride, $384 \mu \mathrm{L}$ (2.7 mmol, 1.5 eq.) of trimethylamine and a catalytic amount of DMAP. The mixture was stirred at RT for $12 \mathrm{~h}$ then washed with $30 \mathrm{~mL}$ of a saturated sodium carbonate solution. The aqueous layer was extracted with $2 \times 30 \mathrm{~mL}$ of DCM. Combined organic layers were washed with brine, dried over magnesium sulfate and concentrated under vacuum. The crude product was purified by silica gel column chromatography (eluent : $\mathrm{DCM} / \mathrm{MeOH} / \mathrm{NH}_{4} \mathrm{OH}: 97 / 2 / 1$ ) to afford the title compound as an amorphous white solid (1.125 g, yield: $97 \%)$. IR $\left(\mathrm{cm}^{-1}\right): 3364,2969,2932,2871,2823,1711$, 1672, 1524, 1276, $1164 ;{ }^{1} \mathrm{H}$ NMR $\left(300 \mathrm{MHz}, \mathrm{CDCl}_{3}\right): \delta(\mathrm{ppm}) 8.04(2 \mathrm{H}, \mathrm{m}, \mathrm{H} 33+\mathrm{H} 37), 7.55$ $(1 \mathrm{H}, \mathrm{m}, \mathrm{H} 35), 7.43(2 \mathrm{H}, \mathrm{m}, \mathrm{H} 34+\mathrm{H} 36), 5.42(1 \mathrm{H}, \mathrm{dd}, \mathrm{J}=8.0 \mathrm{~Hz}, \mathrm{~J}=5.7 \mathrm{~Hz}, \mathrm{H} 16 \beta), 4.47(1 \mathrm{H}, \mathrm{d}$, $\left.\mathrm{J}=9.1 \mathrm{~Hz}, \mathrm{~N}_{\mathrm{A}}-\mathrm{H}\right), 3.70(1 \mathrm{H}, \mathrm{ddd}, \mathrm{J}=12.6 \mathrm{~Hz}, \mathrm{~J}=9.1 \mathrm{~Hz}, \mathrm{~J}=4.0 \mathrm{~Hz}, \mathrm{H} 3 \alpha), 3.34\left(1 \mathrm{H}, \mathrm{d}, \mathrm{J}_{\mathrm{gem}}=\right.$ $12.6 \mathrm{~Hz}, \mathrm{H} 29 \mathrm{~b}), 3.13\left(1 \mathrm{H}, \mathrm{d}, \mathrm{J}_{\mathrm{gem}}=12.6 \mathrm{~Hz}, \mathrm{H} 29 \mathrm{a}\right), 2.62\left(1 \mathrm{H}, \mathrm{d}, \mathrm{J}_{\mathrm{gem}}=17.0 \mathrm{~Hz}, \mathrm{H} 12 \alpha\right), 2.56$ (1H, m, H20), $2.44(1 \mathrm{H}, \mathrm{m}, \mathrm{H} 17 \alpha), 2.42\left(1 \mathrm{H}, \mathrm{d}, \mathrm{J}_{\mathrm{gem}}=17.0 \mathrm{~Hz}, \mathrm{H} 12 \beta\right), 2.41(1 \mathrm{H}, \mathrm{m}, \mathrm{H} 1 \beta), 2.17$ $(1 \mathrm{H}, \mathrm{m}, \mathrm{H} 15 \beta), 2.11\left(6 \mathrm{H}, \mathrm{bs}, \mathrm{N}_{\mathrm{B}} \mathrm{Me}_{2}\right), 2.08(2 \mathrm{H}, \mathrm{m}, \mathrm{H} 5 \alpha+\mathrm{H} 8 \beta), 1.71(1 \mathrm{H}, \mathrm{m}, \mathrm{H} 2 \alpha), 1.69(1 \mathrm{H}$, m, H6 $\alpha), 1.57$ ( $2 \mathrm{H}, \mathrm{m}, \mathrm{H} 15 \alpha+\mathrm{H} 19 \alpha), 1.53(3 \mathrm{H}, \mathrm{m}, \mathrm{H} 2 \beta+\mathrm{H} 7), 1.45\left(9 \mathrm{H}, \mathrm{s}, \mathrm{Me}_{3}\right), 1.26(1 \mathrm{H}, \mathrm{m}$, $\mathrm{H} 1 \alpha), 1.24(3 \mathrm{H}, \mathrm{s}, \mathrm{H} 28), 1.06\left(1 \mathrm{H}, \mathrm{d}, \mathrm{J}_{\mathrm{gem}}=3.6 \mathrm{~Hz}, \mathrm{H} 19 \beta\right), 0.91(3 \mathrm{H}, \mathrm{s}, \mathrm{H} 18), 0.90(1 \mathrm{H}, \mathrm{m}$, $\mathrm{H} 6 \beta), 0.87\left(3 \mathrm{H}, \mathrm{d}, \mathrm{J}_{21-20}=6.3 \mathrm{~Hz}, \mathrm{H} 21\right), 0.56(3 \mathrm{H}, \mathrm{s}, \mathrm{H} 30) ;{ }^{13} \mathrm{C} \mathrm{NMR}\left(75.4 \mathrm{MHz}, \mathrm{CDCl}_{3}\right)$ : $\delta(\mathrm{ppm}) 211.2$ (C11), 165.9 (C31), 157.2 (C1'), 132.5 (C35), 130.8 (C32), 129.4 (C33+C37), 128.2 (C34+C36), 80.1 (C2'), 79.5 (C16), 64.2 (C29), 59.5 (C20), 55.3 (C17), 52.0 (C3), 52.0 (C12), 47.5 (C14), 44.7 (C4+C13), 43.2 (C15), 41.4+41.1 (C5+C8), $40.2\left(\mathrm{~N}_{\mathrm{B}} \mathrm{Me}_{2}\right), 37.9$ (C10), 34.0 (C9), 30.8 (C19), 28.3 ( $\mathrm{Me}_{3}$ ), 27.9+27.8 (C1+C2), 24.4 (C7), 19.6 (C28), 18.5 (C6), 17.9 (C18), 11.0 (C30), 10.0 (C21) ; MS (ESI, m/z) : $637.3(\mathrm{M}+\mathrm{H})$; HRMS (ESI, m/z) : calcd for $\mathrm{C}_{38} \mathrm{H}_{57} \mathrm{~N}_{2} \mathrm{O}_{6}: 637.4216$, found : 637.4191 . 


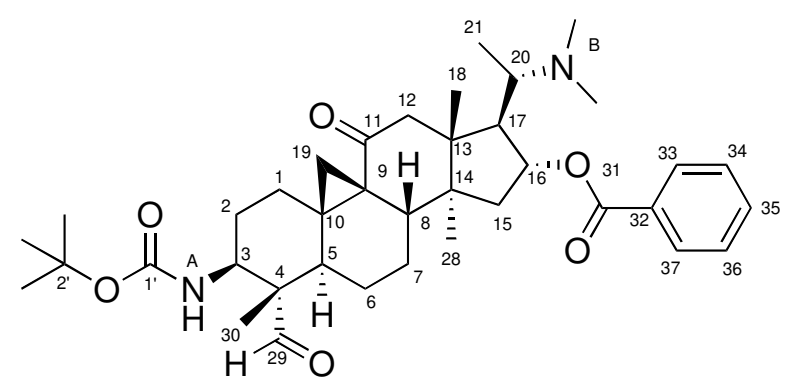

To $1.125 \mathrm{~g}$ ( $1.77 \mathrm{mmol}, 1 \mathrm{eq}$.) of $\mathbf{2 2}$ dissolved in $12 \mathrm{~mL}$ of moist DCM were added $974 \mathrm{mg}$ ( $2.296 \mathrm{mmol}, 1.3$ eq.) of Dess-Martin periodinane. The mixture was stirred at RT for $3 \mathrm{~h}$, diluted with $50 \mathrm{~mL}$ of DCM and washed with $50 \mathrm{~mL}$ of water. The aqueous layer was extracted with $2 \times 30 \mathrm{~mL}$ of DCM. Combined organic layers were washed with brine, dried over magnesium sulfate and concentrated under vacuum to afford the title compound as an amorphous white solid (981 mg, yield: $88 \%$ ). IR $\left(\mathrm{cm}^{-1}\right): 3374,2974,2933,2872,2823,1704$, 1667, 1516, 1278, $1157 ;{ }^{1} \mathrm{H}$ NMR $\left(300 \mathrm{MHz}, \mathrm{CDCl}_{3}\right): \delta(\mathrm{ppm}) 9.42(1 \mathrm{H}, \mathrm{s}, \mathrm{H} 29), 8.04(2 \mathrm{H}, \mathrm{m}$, $\mathrm{H} 33+\mathrm{H} 37), 7.55(1 \mathrm{H}, \mathrm{m}, \mathrm{H} 35), 7.44(2 \mathrm{H}, \mathrm{m}, \mathrm{H} 34+\mathrm{H} 36), 5.41(1 \mathrm{H}, \mathrm{dd}, \mathrm{J}=8.0 \mathrm{~Hz}, \mathrm{~J}=5.5 \mathrm{~Hz}$, $\mathrm{H} 16 \beta), 4.44\left(1 \mathrm{H}, \mathrm{d}, \mathrm{J}=9.3 \mathrm{~Hz}, \mathrm{~N}_{\mathrm{A}}-\mathrm{H}\right), 4.00(1 \mathrm{H}, \mathrm{m}, \mathrm{H} 3 \alpha), 2.64\left(1 \mathrm{H}, \mathrm{d}, \mathrm{J}_{\mathrm{gem}}=17.2 \mathrm{~Hz}, \mathrm{H} 12 \alpha\right)$, $2.52(1 \mathrm{H}, \mathrm{dq}, \mathrm{J}=11.4 \mathrm{~Hz}, \mathrm{~J}=6.1 \mathrm{~Hz}, \mathrm{H} 20), 2.46\left(1 \mathrm{H}, \mathrm{d}, \mathrm{J}_{\mathrm{gem}}=17.2 \mathrm{~Hz}, \mathrm{H} 12 \beta\right), 2.43(2 \mathrm{H}, \mathrm{m}$, $\mathrm{H} 1 \beta+\mathrm{H} 17 \alpha), 2.15(1 \mathrm{H}, \mathrm{m}, \mathrm{H} 15 \beta), 2.10\left(8 \mathrm{H}, \mathrm{m}, \mathrm{N}_{\mathrm{B}} \mathrm{Me}_{2}+\mathrm{H} 5 \alpha+\mathrm{H} 8 \beta\right), 1.86(1 \mathrm{H}, \mathrm{m}, \mathrm{H} 2 \alpha), 1.61$ $(1 \mathrm{H}, \mathrm{d}, \mathrm{J}=4.0 \mathrm{~Hz}, \mathrm{H} 19 \alpha), 1.56(1 \mathrm{H}, \mathrm{d}, \mathrm{J}=14.6 \mathrm{~Hz}, \mathrm{H} 15 \alpha), 1.52(1 \mathrm{H}, \mathrm{m}, \mathrm{H} 2 \beta), 1.49$ (2H, m, H7), $1.41(1 \mathrm{H}, \mathrm{m}, \mathrm{H} 1 \alpha), 1.39\left(9 \mathrm{H}, \mathrm{s}, \mathrm{Me}_{3}\right), 1.27(3 \mathrm{H}, \mathrm{s}, \mathrm{H} 28), 1.07\left(1 \mathrm{H}, \mathrm{d}, \mathrm{J}_{\text {gem }}=4.0 \mathrm{~Hz}, \mathrm{H} 19 \beta\right)$, $1.02(2 \mathrm{H}, \mathrm{m}, \mathrm{H} 6), 0.93(3 \mathrm{H}, \mathrm{s}, \mathrm{H} 30), 0.91(3 \mathrm{H}, \mathrm{s}, \mathrm{H} 18), 0.86\left(3 \mathrm{H}, \mathrm{d}, \mathrm{J}_{21-20}=6.1 \mathrm{~Hz}, \mathrm{H} 21\right) ;{ }^{13} \mathrm{C}$ NMR (75.4 MHz, CDCl ${ }_{3}$ ): $\delta(\mathrm{ppm}) 210.4$ (C11), 203.8 (C29), 165.8 (C31), 154.9 (C1'), 132.5 (C35), 130.7 (C32), 129.4 (C33+C37), 128.2 (C34+C36), 79.7 (C2'), 79.4 (C16), 59.5 (C20), 56.7 (C4), 55.4 (C17), 51.9 (C3), 51.8 (C12), 47.4 (C14), 44.6 (C13), 43.0 (C15), 41.8 (C5), 41.1 (C8), $40.2\left(\mathrm{~N}_{\mathrm{B}} \mathrm{Me}_{2}\right), 35.8(\mathrm{C} 10), 33.5(\mathrm{C} 9), 29.4(\mathrm{C} 19), 28.2\left(\mathrm{Me}_{3}\right), 27.7+27.5(\mathrm{C} 1+\mathrm{C} 2)$, 24.0 (C7), 20.9 (C6), 19.3 (C28), 17.9 (C18), 9.9 (C21), 7.6 (C30) ; MS (ESI, m/z) : 635.3 $(\mathrm{M}+\mathrm{H}) ;$ HRMS (ESI, m/z) : calcd for $\mathrm{C}_{38} \mathrm{H}_{55} \mathrm{~N}_{2} \mathrm{O}_{6}: 635.4060$, found : 635.4053 . 


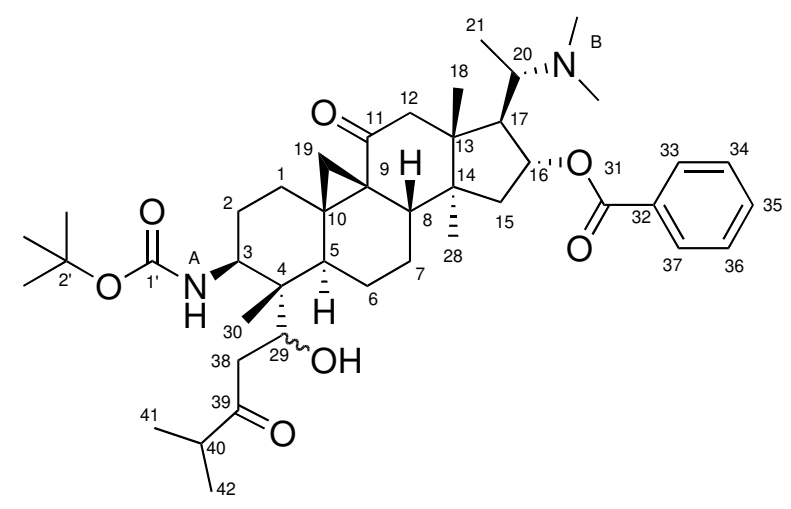

To $126 \mu \mathrm{L}$ (1.17 mmol, 10 eq.) of 3-methyl-2-butanone dissolved in $1 \mathrm{~mL}$ of THF were added at $-78^{\circ} \mathrm{C} 587 \mu \mathrm{L}(1.17 \mathrm{mmol}, 10$ eq.) of LDA (2M in THF/heptane/ethylbenzene). The mixture was stirred for $15 \mathrm{~min}$ then slowly added to $75 \mathrm{mg}(0.117 \mathrm{mmol}, 1$ eq. $)$ of 23 dissolved in $0.5 \mathrm{~mL}$ of THF at $-78^{\circ} \mathrm{C}$. The mixture was stirred at $-10{ }^{\circ} \mathrm{C}$ for $3 \mathrm{~h}$, diluted with $10 \mathrm{~mL}$ of a saturated sodium hydrogen carbonate solution and extracted with $3 \times 10 \mathrm{~mL}$ of DCM. Combined organic layers were washed with brine, dried over magnesium sulfate and concentrated under vacuum., over magnesium sulfate and concentrated under vacuum. The crude product was purified by silica gel column chromatography (eluent : $\mathrm{DCM} / \mathrm{MeOH} / \mathrm{NH}_{4} \mathrm{OH}: 96 / 3 / 1$ ) to afford the title compound as an amorphous light yellow solid $(67 \mathrm{mg}$, yield: $79 \%)$. IR $\left(\mathrm{cm}^{-1}\right): 3364$, 2969, 2932, 2872, 2823, 1708, 1670, 1518, 1278, $1172 ;{ }^{1} \mathrm{H}$ NMR (300 MHz, $\left.\mathrm{CDCl}_{3}\right): \delta(\mathrm{ppm})$ $8.04(2 \mathrm{H}, \mathrm{m}, \mathrm{H} 33+\mathrm{H} 37), 7.55(1 \mathrm{H}, \mathrm{m}, \mathrm{H} 35), 7.44(2 \mathrm{H}, \mathrm{m}, \mathrm{H} 34+\mathrm{H} 36), 5.41(1 \mathrm{H}, \mathrm{dd}, \mathrm{J}=7.8 \mathrm{~Hz}$, $\mathrm{J}=5.6 \mathrm{~Hz}, \mathrm{H} 16 \beta), 4.42\left(1 \mathrm{H}, \mathrm{d}, \mathrm{J}=10.0 \mathrm{~Hz}, \mathrm{~N}_{\mathrm{A}}-\mathrm{H}\right), 4.04(1 \mathrm{H}, \mathrm{d}, \mathrm{J}=9.3 \mathrm{~Hz}, \mathrm{H} 29), 3.61(1 \mathrm{H}, \mathrm{m}$, $\mathrm{H} 3 \alpha), 3.37(1 \mathrm{H}, \mathrm{bs}, \mathrm{OH}), 3.08(1 \mathrm{H}, \mathrm{d}, \mathrm{J}=16.8 \mathrm{~Hz}, \mathrm{H} 38 \mathrm{a}), 2.65(1 \mathrm{H}, \mathrm{sept}, \mathrm{J}=6.9 \mathrm{~Hz}, \mathrm{H} 40), 2.61$ $\left(1 \mathrm{H}, \mathrm{d}, \mathrm{J}_{\mathrm{gem}}=17.0 \mathrm{~Hz}, \mathrm{H} 12 \alpha\right), 2.51(1 \mathrm{H}, \mathrm{m}, \mathrm{H} 20), 2.45(1 \mathrm{H}, \mathrm{m}, \mathrm{H} 17 \alpha), 2.44\left(1 \mathrm{H}, \mathrm{d}, \mathrm{J}_{\mathrm{gem}}=\right.$

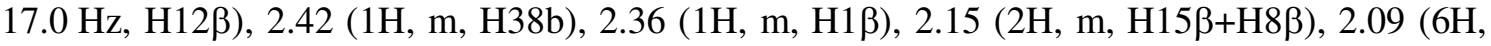
bs, $\left.\mathrm{N}_{\mathrm{B}} \mathrm{Me}_{2}\right), 1.97(1 \mathrm{H}, \mathrm{m}, \mathrm{H} 6 \alpha), 1.80(1 \mathrm{H}, \mathrm{m}, \mathrm{H} 5 \alpha), 1.72(1 \mathrm{H}, \mathrm{m}, \mathrm{H} 2 \alpha), 1.60(1 \mathrm{H}, \mathrm{d}, \mathrm{J}=3.6 \mathrm{~Hz}$,

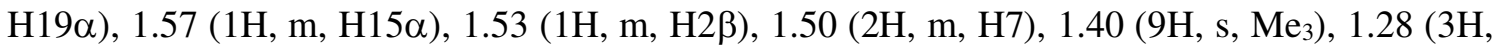
s, H28), $1.24(1 \mathrm{H}, \mathrm{m}, \mathrm{H} 1 \alpha), 1.13(1 \mathrm{H}, \mathrm{m}, \mathrm{H} 19 \beta), 1.10+1.09(6 \mathrm{H}, 2 \mathrm{~d}, \mathrm{~J}=6.9 \mathrm{~Hz}, \mathrm{H} 41+\mathrm{H} 42)$, $1.00(1 \mathrm{H}, \mathrm{m}, \mathrm{H} 6 \beta), 0.91(3 \mathrm{H}, \mathrm{s}, \mathrm{H} 18), 0.88(3 \mathrm{H}, \mathrm{s}, \mathrm{H} 30), 0.85\left(3 \mathrm{H}, \mathrm{d}, \mathrm{J}_{21-20}=6.0 \mathrm{~Hz}, \mathrm{H} 21\right) ;{ }^{13} \mathrm{C}$ NMR (75.4 MHz, $\mathrm{CDCl}_{3}$ ): $\delta(\mathrm{ppm}) 216.3$ (C39), 211.1 (C11), 165.8 (C31), $155.7(\mathrm{C} 1$ '), 132.5 (C35), 130.9 (C32), 129.4 (C33+C37), 128.2 (C34+C36), 79.6 (C2'), 79.5 (C16), 71.5 (C29), 59.4 (C20), 55.4 (C17), 52.7 (C3), 52.0 (C12), 47.5 (C14), 46.1 (C4), 44.6 (C13), 43.9 (C5), 43.1 (C15), 42.0 (C38), 41.3 (C8), 41.0 (C40), $40.2\left(\mathrm{~N}_{\mathrm{B}} \mathrm{Me}_{2}\right), 38.0$ (C10), 34.1 (C9), 30.6 (C19), 29.2 (C2), $28.3\left(\mathrm{Me}_{3}\right), 27.8$ (C1), 24.4 (C7), 19.9 (C6), 19.5 (C28), 18.1+18.0 (C41+C42), 17.9 (C18), 11.5 (C30), 9.9 (C21) ; MS (ESI, m/z) : $721.4(\mathrm{M}+\mathrm{H})$; HRMS (ESI, m/z) : calcd for $\mathrm{C}_{43} \mathrm{H}_{65} \mathrm{~N}_{2} \mathrm{O}_{7}: 721.4792$, found : 721.4763 . 
tert-butyl [20S-(dimethylamino)-16 $\alpha$-hydroxy-4 $\beta, 14 \alpha$-dimethyl-4 $\alpha-[(1 \mathrm{E})-4$-methyl-3oxopent-1-en-1-yl]-11-oxo-9,19-cyclo-5 $\alpha, 9 \beta$-pregnan-3 $\beta$-yl]carbamate (26)

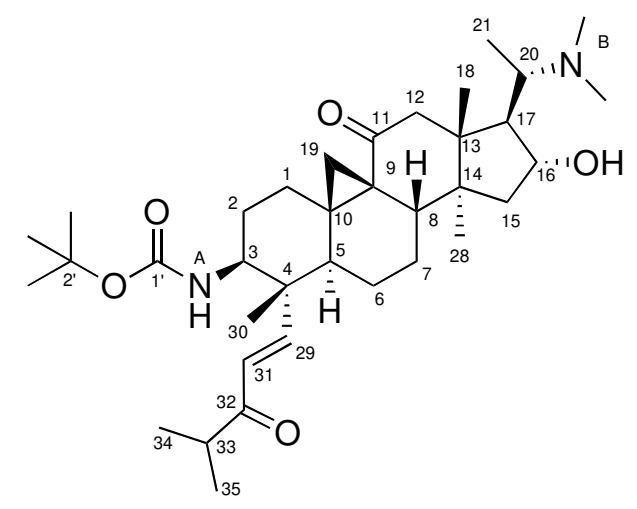

To $89 \mathrm{mg}$ ( $0.123 \mathrm{mmol}, 1$ eq.) of $\mathbf{2 4}$ dissolved in $20 \mathrm{~mL}$ of a mixture $\mathrm{MeOH} /$ water (7/3) was added a small amount of potassium carbonate. The mixture was refluxed for $12 \mathrm{~h}$ then the $\mathrm{MeOH}$ was removed under vacuum. The mixture was diluted with $10 \mathrm{~mL}$ of a saturated sodium carbonate solution and extracted with $3 \times 20 \mathrm{~mL}$ of DCM. Combined organic layers were washed with brine, dried over magnesium sulfate and concentrated under vacuum. The crude product was purified by silica gel column chromatography (eluent : $\mathrm{DCM} / \mathrm{MeOH} / \mathrm{NH}_{4} \mathrm{OH}: 96 / 3 / 1$ ) to afford the title compound as an amorphous colourless solid (64 mg, yield: $86 \%)$. IR $\left(\mathrm{cm}^{-1}\right)$ : 3325, 2970, 2933, 2870, 2836, 1698, 1665, 1520, 1250, $1164 ;{ }^{1} \mathrm{H}$ NMR (500 MHz, $\left.\mathrm{CDCl}_{3}\right)$ : $\delta(\mathrm{ppm}) 6.72(1 \mathrm{H}, \mathrm{d}, \mathrm{J}=16.5 \mathrm{~Hz}, \mathrm{H} 29), 5.92(1 \mathrm{H}, \mathrm{d}, \mathrm{J}=16.5 \mathrm{~Hz}, \mathrm{H} 31), 4.39(1 \mathrm{H}, \mathrm{d}, \mathrm{J}=9.5 \mathrm{~Hz}$, $\left.\mathrm{N}_{\mathrm{A}}-\mathrm{H}\right), 4.10(1 \mathrm{H}, \mathrm{m}, \mathrm{H} 16 \beta), 3.69(1 \mathrm{H}, \mathrm{m}, \mathrm{H} 3 \alpha), 3.07(1 \mathrm{H}, \mathrm{m}, \mathrm{H} 33), 2.62(1 \mathrm{H}, \mathrm{dq}, \mathrm{J}=10.7 \mathrm{~Hz}$, $\mathrm{J}=6.4 \mathrm{~Hz}, \mathrm{H} 20), 2.52\left(1 \mathrm{H}, \mathrm{d}, \mathrm{J}_{\mathrm{gem}}=17.1 \mathrm{~Hz}, \mathrm{H} 12 \alpha\right), 2.38(1 \mathrm{H}, \mathrm{dm}, \mathrm{J}=14 \mathrm{~Hz}, \mathrm{H} 1 \beta), 2.32(1 \mathrm{H}$, $\left.\mathrm{d}, \mathrm{J}_{\mathrm{gem}}=17.1 \mathrm{~Hz}, \mathrm{H} 12 \beta\right), 2.25\left(6 \mathrm{H}, \mathrm{bs}, \mathrm{N}_{\mathrm{B}} \mathrm{Me}_{2}\right), 2.07(1 \mathrm{H}, \mathrm{dd}, \mathrm{J}=10.4 \mathrm{~Hz}, \mathrm{~J}=7.6 \mathrm{~Hz}, \mathrm{H} 8 \beta)$, $2.00(1 \mathrm{H}, \mathrm{m}, \mathrm{H} 15 \beta), 1.98(1 \mathrm{H}, \mathrm{m}, \mathrm{H} 17 \alpha), 1.80(1 \mathrm{H}, \mathrm{m}, \mathrm{H} 2 \alpha), 1.77(1 \mathrm{H}, \mathrm{m}, \mathrm{H} 5 \alpha), 1.60(1 \mathrm{H}, \mathrm{d}$, $\mathrm{J}=3.7 \mathrm{~Hz}, \mathrm{H} 19 \alpha), 1.50(3 \mathrm{H}, \mathrm{m}, \mathrm{H} 2 \beta+\mathrm{H} 7), 1.48(1 \mathrm{H}, \mathrm{dd}, \mathrm{J}=14.3 \mathrm{~Hz}, \mathrm{~J}=2.4 \mathrm{~Hz}, \mathrm{H} 15 \alpha), 1.35$ $(1 \mathrm{H}, \mathrm{m}, \mathrm{H} 1 \alpha), 1.34\left(9 \mathrm{H}, \mathrm{s}, \mathrm{Me}_{3}\right), 1.25(1 \mathrm{H}, \mathrm{m}, \mathrm{H} 6 \alpha), 1.23(3 \mathrm{H}, \mathrm{s}, \mathrm{H} 28), 1.10+1.09(6 \mathrm{H}, 2 \mathrm{~d}, \mathrm{~J}=$

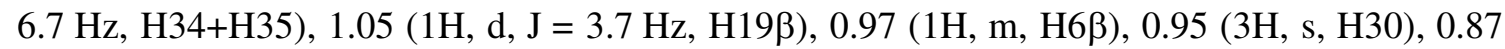
$\left(3 \mathrm{H}, \mathrm{d}, \mathrm{J}_{21-20}=6.4 \mathrm{~Hz}, \mathrm{H} 21\right), 0.83(3 \mathrm{H}, \mathrm{s}, \mathrm{H} 18) ;{ }^{13} \mathrm{C} \mathrm{NMR}\left(75.4 \mathrm{MHz}, \mathrm{CDCl}_{3}\right): \delta(\mathrm{ppm}) 210.8$ (C11), 204.9 (C32), 155.0 (C29), 154.2 (C1'), 128.7 (C31), 79.1 (C2'), 78.2 (C16), 62.0 (C20), 55.8 (C3+C17), 51.4 (C12), 47.8 (C14), 47.1 (C4), 46.8 (C5), 44.5 (C13), 42.6 (C15), 41.2 (C8), 36.6 (C10), 36.4 (C33), 34.4 (C9), 29.7 (C19), 28.2 (Me 3 ), 27.7 (C1+C2), 24.2 (C7), 20.7+20.6 (C34+C35), 17.8 (C28), 18.6 (C6), 17.8 (C18), 10.8 (C30), 9.9 (C21) ; MS (ESI, m/z) : 599.4 $(\mathrm{M}+\mathrm{H}), \mathrm{HRMS}(\mathrm{ESI}, \mathrm{m} / \mathrm{z})$ : calcd for $\mathrm{C}_{36} \mathrm{H}_{59} \mathrm{~N}_{2} \mathrm{O}_{5}: 599.4424$, found : 599.4454 . 
$N$-[20S-(dimethylamino)-16 $\alpha$-hydroxy-4 $\alpha$-(mercaptomethyl)-4 $\beta, 14 \alpha$-dimethyl-11oxo-9, 19-cyclo-5 $\alpha, 9 \beta$-pregnan-3 $\beta$-yl]-isobutyramide (29).

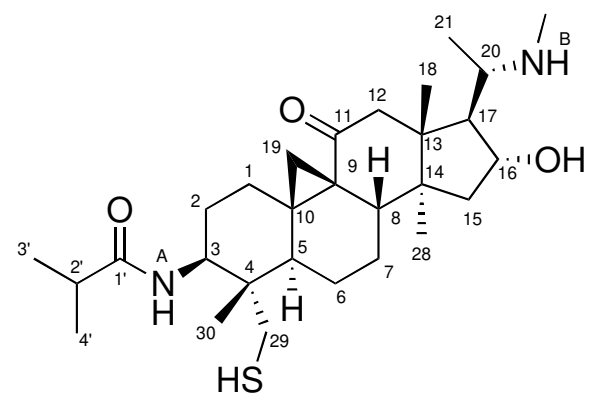

To $40 \mathrm{mg}$ ( $0.057 \mathrm{mmol}, 1$ eq.) of $\mathbf{2 8}$ dissolved in $0.5 \mathrm{~mL}$ of DMPU were added $98 \mathrm{mg}$ $(0.86 \mathrm{mmol}, 15$ eq. $)$ of potassium thioacetate. The mixture was stirred at $100{ }^{\circ} \mathrm{C}$ for $5 \mathrm{~h}$ then diluted with $10 \mathrm{~mL}$ of a saturated sodium hydrogen carbonate solution and extracted with $20 \mathrm{~mL}$ of $\mathrm{Et}_{2} \mathrm{O}$. The organic layer was dried with brine, over magnesium sulfate and concentrated under vacuum. The residue was dissolved in $20 \mathrm{~mL}$ of a degassed mixture $\mathrm{MeOH} /$ water (7/3) and a small amount of potassium hydroxide was added. The mixture was stirred at RT for $3 \mathrm{~h}$. The $\mathrm{MeOH}$ was removed under reduced pressure and the mixture was extracted with $3 \times 20 \mathrm{~mL}$ of DCM. Combined organic layers were washed with brine, dried over magnesium sulfate and concentrated under vacuum. The crude product was purified by silica gel column chromatography (gradient elution : $\mathrm{DCM} / \mathrm{MeOH} / \mathrm{NH}_{4} \mathrm{OH}$ : from $99 / 0 / 1$ to $90 / 10 / 1$ ) to afford the title compound as an amorphous white solid (20 mg, yield: $67 \%$ ). IR $\left(\mathrm{cm}^{-1}\right): 3322,2964,2937,2869$, 2833, 1655, 1534, 1460, 1225, 1159, $1094 ;{ }^{1} \mathrm{H}$ NMR (300 MHz, $\left.\mathrm{CDCl}_{3}\right): \delta(\mathrm{ppm}) 5.23(1 \mathrm{H}$, $\left.\mathrm{d}, \mathrm{J}_{\mathrm{NAH}-3}=9.9 \mathrm{~Hz}, \mathrm{~N}_{\mathrm{A}}-\mathrm{H}\right), 4.15(1 \mathrm{H}, \mathrm{m}, \mathrm{H} 3 \alpha), 4.12(1 \mathrm{H}, \mathrm{m}, \mathrm{H} 16 \beta), 2.64\left(1 \mathrm{H}, \mathrm{dq}, \mathrm{J}_{20-17}=\right.$ $\left.10.8 \mathrm{~Hz}, \mathrm{~J}_{20-21}=6.5 \mathrm{~Hz}, \mathrm{H} 20\right), 2.53\left(1 \mathrm{H}, \mathrm{d}, \mathrm{J}_{\mathrm{gem}}=17.1 \mathrm{~Hz}, \mathrm{H} 12 \alpha\right), 2.49$ (1H, m, H29b), $2.41(1 \mathrm{H}, \mathrm{m}, \mathrm{H} 1 \beta), 2.34\left(1 \mathrm{H}, \mathrm{m}, \mathrm{H} 2\right.$ ') $2.29\left(1 \mathrm{H}, \mathrm{d}, \mathrm{J}_{\mathrm{gem}}=17.1 \mathrm{~Hz}, \mathrm{H} 12 \beta\right), 2.26(6 \mathrm{H}, \mathrm{bs}$, $\mathrm{N}_{\mathrm{BMe}}$ ), 2.22 (1H, m, H5), 2.19 (1H, m, H29a), 2.02 (2H, m, H8 $\left.\beta+\mathrm{H} 15 \beta\right), 1.99$ (1H, m, $\mathrm{H} 17 \alpha), 1.72(1 \mathrm{H}, \mathrm{m}, \mathrm{H} 2 \alpha), 1.56\left(1 \mathrm{H}, \mathrm{d}, \mathrm{J}_{\mathrm{gem}}=3.4 \mathrm{~Hz}, \mathrm{H} 19 \alpha\right), 1.55(1 \mathrm{H}, \mathrm{m}, \mathrm{H} 7 \beta), 1.51$

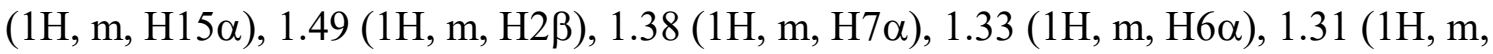
$\mathrm{H} 1 \alpha), 1.23(3 \mathrm{H}, \mathrm{s}, \mathrm{H} 28), 1.17+1.14\left(6 \mathrm{H}, 2 \mathrm{~d}, \mathrm{~J}=7.2 \mathrm{~Hz}, \mathrm{H} 3{ }^{\prime}+\mathrm{H} 4{ }^{\prime}\right), 1.03\left(1 \mathrm{H}, \mathrm{d}, \mathrm{J}_{\text {gem }}=\right.$ $3.4 \mathrm{~Hz}, \mathrm{H} 19 \beta), 0.92$ (1H, m, H6ß), 0.89 (3H, d, J $21-20=6.5 \mathrm{~Hz}, \mathrm{H} 21), 0.85$ (3H, s, H18), $0.78(3 \mathrm{H}, \mathrm{s}, \mathrm{H} 30) ;{ }^{13} \mathrm{C}$ NMR $\left(75.4 \mathrm{MHz}, \mathrm{CDCl}_{3}\right): \delta(\mathrm{ppm}) 211.4(\mathrm{C} 11), 176.7(\mathrm{C} 1$ '), 78.1 (C16), 62.0 (C20), 55.7 (C17), 51.4 (C12), 49.7 (C3), 47.0 (C14), 44.4 (C13), 43.1 (C4), 42.9 (C15), 42.0 (C5), 41.9 (C8), 37.6 (C10), 35.8 (C2'), 34.2 (C9), 31.2 (C29), 30.9 (C19), 28.5 (C2), 27.4 (C1), 24.3 (C7), 20.8 (C28), 20.1+19.4 (C3'+C4'), 18.4 (C6), 17.8 (C18), 15.5 (C30), 9.9 (C21) ; MS (ESI, m/z) : $519.3(\mathrm{M}+\mathrm{H})$; HRMS (ESI, m/z) : calcd for $\mathrm{C}_{30} \mathrm{H}_{51} \mathrm{~N}_{2} \mathrm{O}_{3} \mathrm{~S}: 519.3620$, found : 519.3611 . 


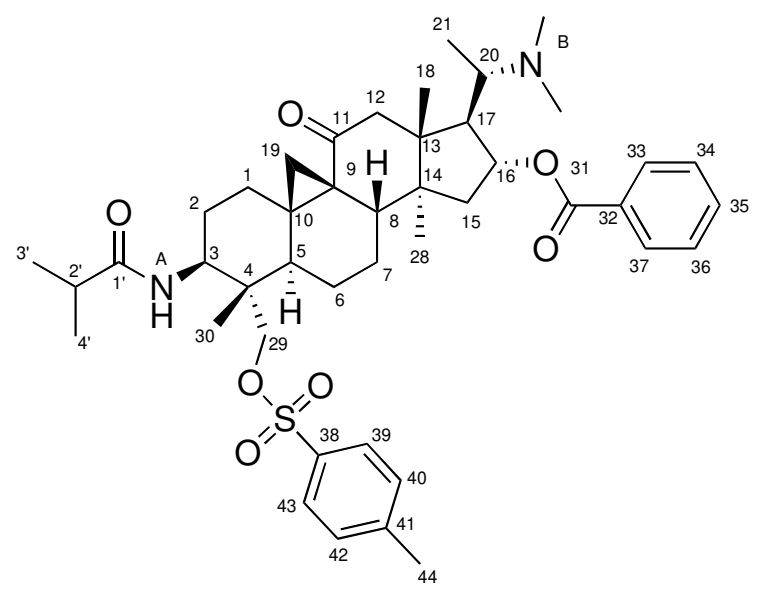

To $747 \mathrm{mg}$ (1.2 mmol, 1 eq.) of $\mathbf{1 4}$ dissolved in $30 \mathrm{~mL}$ of THF were added $234 \mathrm{mg}$ ( $1.2 \mathrm{mmol}, 1$ eq.) of $p$-toluene sulfonic acid. The mixture was stirred at RT for $30 \mathrm{~min}$ then concentrated under reduced pressure. The residue was dissolved in $7 \mathrm{~mL}$ of pyridine and $1.173 \mathrm{~g}$ (6.1 mmol, 5 eq.) of tosyl chloride were added. The mixture was stirred at $90{ }^{\circ} \mathrm{C}$ for $3 \mathrm{~h}$. After 3 co-evaporations with $3 \times 20 \mathrm{~mL}$ of 1,2-dichloroethane the residue was dissolved in DCM, washed with $30 \mathrm{~mL}$ of a saturated sodium hydrogen carbonate solution and extracted with $3 \times 30 \mathrm{~mL}$ of DCM. Combined organic layers were washed with brine, dried over magnesium sulfate and concentrated under vacuum. The crude product was purified by silica gel column chromatography (eluent : $\mathrm{DCM} / \mathrm{MeOH} / \mathrm{NH}_{4} \mathrm{OH}$ : 97/2/1) to afford the title compound as an amorphous light yellow solid (805 mg, yield: $86 \%$ ). IR : 3322, 2964, 2929, 2869, 2823, 1711, $1666,1529,1600,1450,1359,1278,1228,1188,1175 ;{ }^{1} \mathrm{H}$ NMR $\left(300 \mathrm{MHz}, \mathrm{CDCl}_{3}\right): \delta(\mathrm{ppm})$ $8.05(2 \mathrm{H}, \mathrm{m}, \mathrm{H} 33+\mathrm{H} 37), 7.78(2 \mathrm{H}, \mathrm{d}, \mathrm{J}=8.3 \mathrm{~Hz}, \mathrm{H} 39+\mathrm{H} 43), 7.56(1 \mathrm{H}, \mathrm{m}, \mathrm{H} 35), 7.45(2 \mathrm{H}, \mathrm{m}$, $\mathrm{H} 34+\mathrm{H} 36), 7.30(2 \mathrm{H}, \mathrm{d}, \mathrm{J}=8.3 \mathrm{~Hz}, \mathrm{H} 40+\mathrm{H} 42), 5.42(1 \mathrm{H}, \mathrm{dd}, \mathrm{J}=8.1 \mathrm{~Hz}, \mathrm{~J}=5.7 \mathrm{~Hz}, \mathrm{H} 16 \beta)$, $5.30\left(1 \mathrm{H}, \mathrm{d}, \mathrm{J}_{\mathrm{NAH}-\mathrm{H} 3}=10.0 \mathrm{~Hz}, \mathrm{~N}_{\mathrm{A}}-\mathrm{H}\right), 4.09(1 \mathrm{H}, \mathrm{m}, \mathrm{H} 3 \alpha), 3.80\left(1 \mathrm{H}, \mathrm{d}, \mathrm{J}_{\mathrm{gem}}=9.6 \mathrm{~Hz}, \mathrm{H} 29 \mathrm{~b}\right)$, $3.55\left(1 \mathrm{H}, \mathrm{d}, \mathrm{J}_{\mathrm{gem}}=9.6 \mathrm{~Hz}, \mathrm{H} 29 \mathrm{a}\right), 2.62\left(1 \mathrm{H}, \mathrm{d}, \mathrm{J}_{\mathrm{gem}}=17.3 \mathrm{~Hz}, \mathrm{H} 12 \alpha\right), 2.54\left(1 \mathrm{H}, \mathrm{dq}, \mathrm{J}_{20-17 \alpha}=\right.$ $\left.11.3 \mathrm{~Hz}, \mathrm{~J}_{20-21}=6.0 \mathrm{~Hz}, \mathrm{H} 20\right), 2.45\left(1 \mathrm{H}, \mathrm{dd}, \mathrm{J}_{17 \alpha-20}=11.3 \mathrm{~Hz}, \mathrm{~J}_{17 \alpha-16 \beta}=5.7 \mathrm{~Hz}, \mathrm{H} 17 \alpha\right), 2.43(1 \mathrm{H}$, $\left.\mathrm{d}, \mathrm{J}_{\text {gem }}=17.3 \mathrm{~Hz}, \mathrm{H} 12 \beta\right), 2.39(3 \mathrm{H}, \mathrm{s}, \mathrm{H} 37), 2.36(1 \mathrm{H}, \mathrm{m}, \mathrm{H} 1 \beta), 2.23(1 \mathrm{H}, \mathrm{m}, \mathrm{H} 2$ '), $2.12(1 \mathrm{H}, \mathrm{m}$, $\mathrm{H} 5 \alpha), 2.10\left(6 \mathrm{H}, \mathrm{bs}, \mathrm{N}_{\mathrm{B}} \mathrm{Me}_{2}\right), 2.03(1 \mathrm{H}, \mathrm{m}, \mathrm{H} 8 \beta), 2.01(1 \mathrm{H}, \mathrm{d}, \mathrm{J}=12.2 \mathrm{~Hz}, \mathrm{H} 15 \beta), 1.71(1 \mathrm{H}, \mathrm{m}$, $\mathrm{H} 2 \alpha), 1.58(1 \mathrm{H}, \mathrm{d}, \mathrm{J}=12.2 \mathrm{~Hz}, \mathrm{H} 15 \alpha), 1.56\left(1 \mathrm{H}, \mathrm{d}, \mathrm{J}_{\mathrm{gem}}=3.4 \mathrm{~Hz}, \mathrm{H} 19 \beta\right), 1.46(3 \mathrm{H}, \mathrm{m}$, $\mathrm{H} 2 \beta+\mathrm{H} 6 \alpha+\mathrm{H} 7 \beta), 1.29(1 \mathrm{H}, \mathrm{m}, \mathrm{H} 1 \alpha), 1.26(1 \mathrm{H}, \mathrm{m}, \mathrm{H} 7 \alpha), 1.23(3 \mathrm{H}, \mathrm{s}, \mathrm{H} 28), 1.04\left(3 \mathrm{H}, \mathrm{d}, \mathrm{J}^{\prime}{ }^{\prime}-{ }^{\prime}{ }^{\prime}=\right.$ $\left.7.0 \mathrm{~Hz}, \mathrm{H} 4^{\prime}\right), 1.03\left(1 \mathrm{H}, \mathrm{d}, \mathrm{J}_{\mathrm{gem}}=3.4 \mathrm{~Hz}, \mathrm{H} 19 \beta\right), 0.98\left(3 \mathrm{H}, \mathrm{d}, \mathrm{J}^{\prime}-4{ }^{\prime}=7.0 \mathrm{~Hz}, \mathrm{H} 3^{\prime}\right), 0.91$ (3H, s, $\mathrm{H} 18), 0.85\left(3 \mathrm{H}, \mathrm{d}, \mathrm{J}_{21-20}=6.0 \mathrm{~Hz}, \mathrm{H} 21\right), 0.84(1 \mathrm{H}, \mathrm{m}, \mathrm{H} 6 \beta), 0.73(3 \mathrm{H}, \mathrm{s}, \mathrm{H} 30) ;{ }^{13} \mathrm{C}$ NMR $(75.4$ $\left.\mathrm{MHz}, \mathrm{CDCl}_{3}\right): \delta(\mathrm{ppm}) 210.8(\mathrm{C} 11), 175.9(\mathrm{C} 1$ ') $, 165.8(\mathrm{C} 31), 144.4(\mathrm{C} 41), 132.5(\mathrm{C} 35), 132.4$ (C38), 130.6 (C32), 129.6 (C40+C42), 129.2 (C33+C37), 128.1 (C34+C36), 128.0 (C39+C43), 79.3 (C16), 71.2 (C29), 59.3 (C20), 55.1 (C17), 51.8 (C12), 49.4 (C3), 47.3 (C14), 44.5 (C13), 43.0 (C15), 42.9 (C4), 42.7 (C5), 41.3 (C8), $40.1\left(\mathrm{~N}_{\mathrm{B}} \mathrm{Me}_{2}\right), 37.1$ (C10), 35.5 (C2'), 33.6 (C9), 30.1 (C19), 28.0 (C2), 27.0 (C1), 24.1 (C7), 21.4 (C37), 19.6 (C3'), 19.4 (C28), 19.2 (C4'), 18.5 (C6), 17.7 (C18), 10.8 (C30), 9.8 (C21); MS (ESI): 761.3 (M+H); HRMS (ESI, m/z): calcd for $\mathrm{C}_{44} \mathrm{H}_{61} \mathrm{~N}_{2} \mathrm{O}_{7} \mathrm{~S}: 761.4199$, found : 761.4197 . 


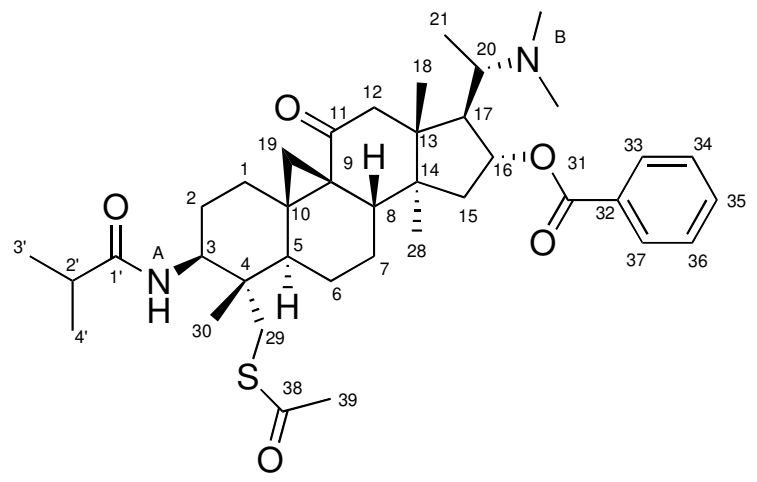

To $805 \mathrm{mg}$ ( $1.06 \mathrm{mmol}, 1$ eq.) of $\mathbf{3 1}$ dissolved in $7.5 \mathrm{~mL}$ of DMPU were added $1.811 \mathrm{mg}$ ( $15.8 \mathrm{mmol}, 15 \mathrm{eq}$.) of potassium thioacetate. The mixture was stirred at $100{ }^{\circ} \mathrm{C}$ for $5 \mathrm{~h}$, diluted with $70 \mathrm{~mL}$ of a saturated sodium hydrogen carbonate solution and extracted with $70 \mathrm{~mL}$ of $\mathrm{Et}_{2} \mathrm{O}$. The organic layer was dried with brine, over magnesium sulfate and concentrated under reduced pressure. The residue was dissolved in $40 \mathrm{~mL}$ of DCM and $261 \mathrm{mg}$ (1.06 mmol, 1 eq.) of $m$-CPBA were added at $0{ }^{\circ} \mathrm{C}$. The mixture was stirred at $0{ }^{\circ} \mathrm{C}$ for $1 \mathrm{~h}$ and $10 \mathrm{~mL}$ of $\mathrm{MeOH}, 285 \mathrm{mg}$ (1.06 mmol, 1 eq.) of iron chloride and $588 \mathrm{mg}$ ( $2.1 \mathrm{mmol}, 2$ eq.) of iron sulfate were added. The mixture was stirred at $10{ }^{\circ} \mathrm{C}$ for further $3 \mathrm{~h}$ and $3 \mathrm{~mL}$ of $\mathrm{NH}_{4} \mathrm{OH}$ were added. The mixture was filtered through a pad of celite and the solvents were removed under vacuum. The crude product was purified by silica gel column chromatography (eluent : $\mathrm{DCM} / \mathrm{MeOH} / \mathrm{NH}_{4} \mathrm{OH}: 96 / 3 / 1$ ) to afford $555 \mathrm{mg}$ of 32 (yield: $81 \%$ ) as an amorphous light yellow solid. IR $\left(\mathrm{cm}^{-1}\right): 3317,2967,2932,2869,2824,1704,1660,1626,1526,1450,1278$, 1228, $\left.1113 ;{ }^{1} \mathrm{H} \mathrm{NMR} \mathrm{(300} \mathrm{MHz,} \mathrm{CDCl}_{3}\right): \delta(\mathrm{ppm}) 8.05(2 \mathrm{H}, \mathrm{m}, \mathrm{H} 33+\mathrm{H} 37), 7.55(1 \mathrm{H}, \mathrm{m}, \mathrm{H} 35)$, $7.44(2 \mathrm{H}, \mathrm{m}, \mathrm{H} 34+\mathrm{H} 36), 5.42\left(1 \mathrm{H}, \mathrm{d}, \mathrm{J}_{\mathrm{NAH}-\mathrm{H} 3}=9.3 \mathrm{~Hz}, \mathrm{~N}_{\mathrm{A}}-\mathrm{H}\right), 5.41(1 \mathrm{H}, \mathrm{m}, \mathrm{H} 16 \beta), 3.95(1 \mathrm{H}$, ddd, $\mathrm{J}=12.2 \mathrm{~Hz}, \mathrm{~J}=10.0 \mathrm{~Hz}, \mathrm{~J}=4.3 \mathrm{~Hz}, \mathrm{H} 3 \alpha), 3.03\left(1 \mathrm{H}, \mathrm{d}, \mathrm{J}_{\mathrm{gem}}=13.8 \mathrm{~Hz}, \mathrm{H} 29 \mathrm{~b}\right), 2.85(1 \mathrm{H}, \mathrm{d}$, $\left.\mathrm{J}_{\mathrm{gem}}=13.8 \mathrm{~Hz}, \mathrm{H} 29 \mathrm{a}\right), 2.62\left(1 \mathrm{H}, \mathrm{d}, \mathrm{J}_{\mathrm{gem}}=17.0 \mathrm{~Hz}, \mathrm{H} 12 \alpha\right), 2.50(1 \mathrm{H}, \mathrm{m}, \mathrm{H} 20), 2.43(2 \mathrm{H}, \mathrm{m}$,

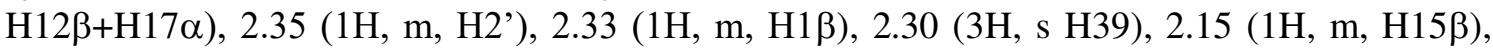
$2.10\left(7 \mathrm{H}, \mathrm{m}, \mathrm{N}_{\mathrm{B}} \mathrm{Me}, \mathrm{H} 8 \beta\right), 1.75(1 \mathrm{H}, \mathrm{m}, \mathrm{H} 5 \alpha), 1.72(1 \mathrm{H}, \mathrm{m}, \mathrm{H} 2 \alpha), 1.60\left(1 \mathrm{H}, \mathrm{d}, \mathrm{J}_{\mathrm{gem}}=4.1 \mathrm{~Hz}\right.$,

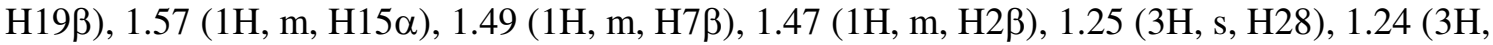
$\mathrm{m}, \mathrm{H} 1 \alpha+\mathrm{H} 6 \alpha+\mathrm{H} 7 \alpha), 1.16+1.14\left(6 \mathrm{H}, 2 \mathrm{~d}, \mathrm{~J}_{3^{\prime}-4^{\prime}}=6.9 \mathrm{~Hz}, \mathrm{H} 3^{\prime}+\mathrm{H}^{\prime}{ }^{\prime}\right), 1.07\left(1 \mathrm{H}, \mathrm{d}, \mathrm{J}_{\mathrm{gem}}=4.1 \mathrm{~Hz}\right.$,

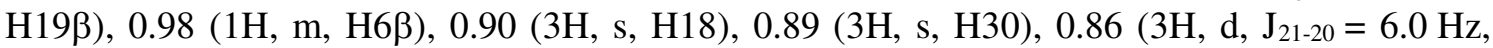
$\mathrm{H} 21) ;{ }^{13} \mathrm{C}$ NMR $\left(75.4 \mathrm{MHz}, \mathrm{CDCl}_{3}\right): \delta(\mathrm{ppm}) 210.7(\mathrm{C} 11), 195.6(\mathrm{C} 38), 176.3(\mathrm{C} 1$ '), 165.7 (C31), 132.5 (C35), 130.7 (C32), 129.3 (C33+C37), 128.2 (C34+C36), 79.4 (C16), 56.4 (C20), 55.3 (C17), 52.0 (C3), 51.8 (C12), 47.4 (C14), 44.6 (C13), 44.5 (C5), 42.9 (C15), 42.8 (C4), 40.9 (C8), 40.2 ( $\left.\mathrm{N}_{\mathrm{B}} \mathrm{Me}\right), 37.6$ (C10), 35.7 (C2'), 35.4 (C29), 33.9 (C9), 30.6 (C39), 29.7 (C19), 28.4 (C2), 27.2 (C1), 24.2 (C7), 19.7 (C28), 19.4 (C3'+C4'), 18.8 (C6), 17.8 (C18), 15.0 (C30), $9.8(\mathrm{C} 21)$; MS (ESI, m/z) : $665.3(\mathrm{M}+\mathrm{H})$; HRMS (ESI, m/z) : calcd for $\mathrm{C}_{39} \mathrm{H}_{57} \mathrm{~N}_{2} \mathrm{O}_{5} \mathrm{~S}: 665.3988$, found : 665.3980 . 


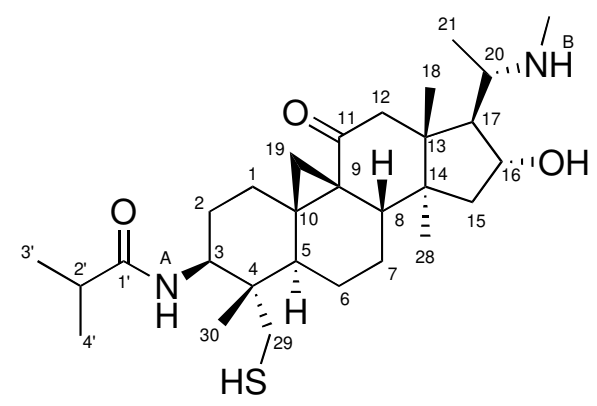

To $240 \mathrm{mg}$ ( $0.24 \mathrm{mmol}, 1$ eq.) of $\mathbf{2 5 4}$ dissolved in $50 \mathrm{~mL}$ of a degassed mixture of $\mathrm{MeOH} / \mathrm{H}_{2} \mathrm{O}$ (4/1) was added a small amount of potassium hydroxide. The mixture was stirred at $\mathrm{RT}$ for $5 \mathrm{~h}$ then $\mathrm{MeOH}$ was removed under vacuum. The aqueous layer was extracted with $3 \times 30 \mathrm{~mL}$ of DCM. Combined organic layers were washed with brine, dried over magnesium sulfate and concentrated under vacuum. The crude product was purified by silica gel column chromatography (gradient elution : $\mathrm{DCM} / \mathrm{MeOH} / \mathrm{NH}_{4} \mathrm{OH}$ : from $99 / 0 / 1$ to $90 / 10 / 1$ ) to afford the title compound as an amorphous white solid (102 mg, yield: $55 \%)$. IR $\left(\mathrm{cm}^{-1}\right): 3315,2964$, 2937, 2869, 1660, 1650, 1537, 1454, 1227, 1156, $1090 ;{ }^{1} \mathrm{H}$ NMR (300 MHz, $\left.\mathrm{CDCl}_{3}\right): \delta(\mathrm{ppm})$ $5.17\left(1 \mathrm{H}, \mathrm{d}, \mathrm{J}_{\mathrm{NAH}-3}=10.1 \mathrm{~Hz}, \mathrm{~N}_{\mathrm{A}}-\mathrm{H}\right), 4.15(1 \mathrm{H}, \mathrm{m}, \mathrm{H} 3 \alpha), 4.14(1 \mathrm{H}, \mathrm{m}, \mathrm{H} 16 \beta), 2.54(1 \mathrm{H}, \mathrm{d}$, $\left.\mathrm{J}_{\mathrm{gem}}=17.1 \mathrm{~Hz}, \mathrm{H} 12 \alpha\right), 2.21(1 \mathrm{H}, \mathrm{m}, \mathrm{H} 29 \mathrm{~b}), 2.47(1 \mathrm{H}, \mathrm{m}, \mathrm{H} 20), 2.45\left(3 \mathrm{H}, \mathrm{s}, \mathrm{N}_{\mathrm{B}} \mathrm{Me}\right), 2.41(1 \mathrm{H}$, $\mathrm{dt}, \mathrm{J}=13.7 \mathrm{~Hz}, \mathrm{~J}=3.5 \mathrm{~Hz}, \mathrm{H} 1 \beta), 2.36\left(1 \mathrm{H}, \mathrm{d}, \mathrm{J}_{\mathrm{gem}}=17.1 \mathrm{~Hz}, \mathrm{H} 12 \beta\right), 2.34\left(1 \mathrm{H}, \mathrm{m}, \mathrm{H} 2{ }^{\prime}\right), 2.23$ $(2 \mathrm{H}, \mathrm{m}, \mathrm{H} 5+\mathrm{H} 29 \mathrm{a}), 2.01(2 \mathrm{H}, \mathrm{m}, \mathrm{H} 8 \beta+\mathrm{H} 15 \beta), 1.73(2 \mathrm{H}, \mathrm{m}, \mathrm{H} 2 \alpha+\mathrm{H} 17 \alpha), 1.56\left(1 \mathrm{H}, \mathrm{d}, \mathrm{J}_{\mathrm{gem}}=\right.$ $3.7 \mathrm{~Hz}, \mathrm{H} 19 \alpha), 1.53(1 \mathrm{H}, \mathrm{m}, \mathrm{H} 7 \beta), 1.52(1 \mathrm{H}, \mathrm{m}, \mathrm{H} 15 \alpha), 1.48(1 \mathrm{H}, \mathrm{m}, \mathrm{H} 2 \beta), 1.39$ (1H, m, H7 $\alpha$ ), $1.33(2 \mathrm{H}, \mathrm{m}, \mathrm{H} 1 \alpha+\mathrm{H} 6 \alpha), 1.21(3 \mathrm{H}, \mathrm{s}, \mathrm{H} 28), 1.17+1.15(6 \mathrm{H}, 2 \mathrm{~d}, \mathrm{~J}=7.0 \mathrm{~Hz}, \mathrm{H} 3$ ' $+\mathrm{H} 4$ '), 1.09 (3H, d, $\left.\mathrm{J}_{21-20}=6.1 \mathrm{~Hz}, \mathrm{H} 21\right), 1.03\left(1 \mathrm{H}, \mathrm{d}, \mathrm{J}_{\mathrm{gem}}=3.7 \mathrm{~Hz}, \mathrm{H} 19 \beta\right), 0.89(1 \mathrm{H}, \mathrm{m}, \mathrm{H} 6 \beta), 0.85(3 \mathrm{H}, \mathrm{s}, \mathrm{H} 18)$, 0.78 (3H, s, H30); ${ }^{13} \mathrm{C}$ NMR (75.4 MHz, $\left.\mathrm{CDCl}_{3}\right)$ : $\delta(\mathrm{ppm}) 211.4$ (C11), 176.7 (C1'), 77.9 (C16), 60.6 (C17), 58.5 (C20), 51.5 (C12), 49.7 (C3), 47.1 (C14), 44.6 (C13), 43.1 (C4), 42.9 (C15), 42.0 (C5), 41.9 (C8), 37.6 (C10), 35.9 (C2'), 34.2 (C9), 33.8 ( $\mathrm{N}_{\mathrm{B}} \mathrm{Me}$ ), 31.2 (C29), 30.9 (C19), 28.6 (C2), 27.5 (C1), 24.4 (C7), 20.7 (C28), 20.1 (C3'), 19.4 (C4'), 18.6 (C21), 18.5 (C6), 18.0 (C18), 15.5 (C30); MS (ESI, m/z) : $505.3(\mathrm{M}+\mathrm{H})$; HRMS (ESI, m/z) : calcd for $\mathrm{C}_{29} \mathrm{H}_{49} \mathrm{~N}_{2} \mathrm{O}_{3} \mathrm{~S}$ : 505.3464, found : 505.3458 . 


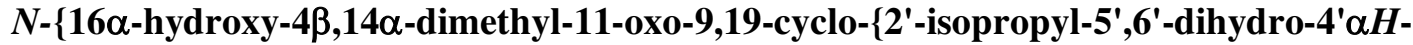 $\left[1^{\prime}, 3^{\prime}\right]$ thiazino $\left.\left[4^{\prime}, 5{ }^{\prime}: 3,4\right]\right\}-5 \alpha, 9 \beta$-pregnan-20S-yl\}- $N$-methylbenzamide (35b)}

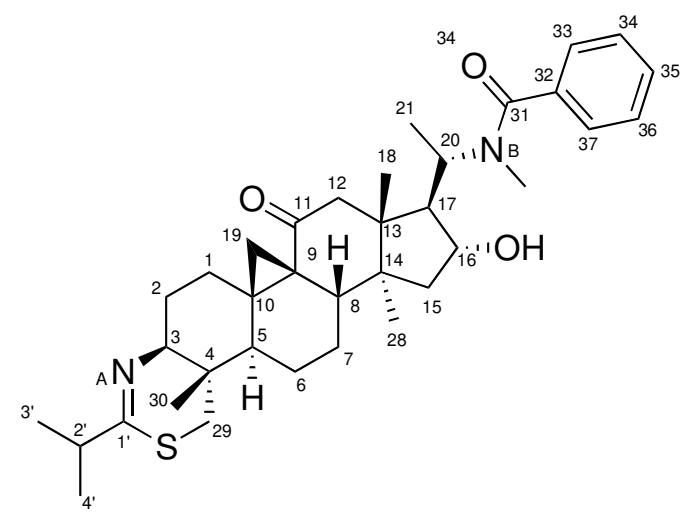

$13 \mathrm{mg}$ ( $0.0267 \mathrm{mmol}, 1$ eq.) of $\mathbf{3 4}, 9 \mathrm{mg}(0.0347 \mathrm{mmol}, 1.3 \mathrm{eq}$.$) of benzoic anhydride$ and $1 \mathrm{~mL}$ of EtOH were used by following similar procedure described for the preparation of 35a to give the title compound as an amorphous white solid $(12.5 \mathrm{mg}$, yield: $83 \%)$. IR $\left(\mathrm{cm}^{-1}\right)$ : $3333,2962,2931,2868,1666,1613,1598,1574,1447,1257,1115,1084,1025 ;{ }^{1} \mathrm{H}$ NMR $(300$ $\left.\mathrm{MHz}, \mathrm{CDCl}_{3}\right): \delta(\mathrm{ppm}) 7.39(5 \mathrm{H}, \mathrm{m}, \mathrm{H} 33+\mathrm{H} 34+\mathrm{H} 35+\mathrm{H} 36+\mathrm{H} 37), 5.14(1 \mathrm{H}, \mathrm{m}, \mathrm{H} 20), 4.41(1 \mathrm{H}$, $\mathrm{m}, \mathrm{H} 16 \beta), 2.88(1 \mathrm{H}, \mathrm{m}, \mathrm{H} 3 \alpha), 2.86\left(3 \mathrm{H}, \mathrm{s}, \mathrm{N}_{\mathrm{B}} \mathrm{Me}\right), 2.84\left(1 \mathrm{H}, \mathrm{d}, \mathrm{J}_{\mathrm{gem}}=11.2 \mathrm{~Hz}, \mathrm{H} 29 \mathrm{~b}\right), 2.75(1 \mathrm{H}$, $\left.\mathrm{d}, \mathrm{J}_{\mathrm{gem}}=11.2 \mathrm{~Hz}, \mathrm{H} 29 \mathrm{a}\right), 2.68\left(1 \mathrm{H}, \mathrm{m}, \mathrm{H} 2^{\prime}\right), 2.60\left(1 \mathrm{H}, \mathrm{d}, \mathrm{J}_{\mathrm{gem}}=17.0 \mathrm{~Hz}, \mathrm{H} 12 \alpha\right), 2.47(1 \mathrm{H}, \mathrm{m}$, $\mathrm{H} 1 \beta), 2.42\left(1 \mathrm{H}, \mathrm{d}, \mathrm{J}_{\mathrm{gem}}=17.0 \mathrm{~Hz}, \mathrm{H} 12 \beta\right), 2.26(1 \mathrm{H}, \mathrm{m}, \mathrm{H} 17 \alpha), 2.09(2 \mathrm{H}, \mathrm{m}, \mathrm{H} 8 \beta+\mathrm{H} 15 \beta), 1.73$ $(1 \mathrm{H}, \mathrm{m}, \mathrm{H} 2 \alpha), 1.67\left(1 \mathrm{H}, \mathrm{d}, \mathrm{J}_{\mathrm{gem}}=4.0 \mathrm{~Hz}, \mathrm{H} 19 \alpha\right), 1.59(1 \mathrm{H}, \mathrm{m}, \mathrm{H} 2 \beta), 1.57(2 \mathrm{H}, \mathrm{m}, \mathrm{H} 7), 1.54$ $(1 \mathrm{H}, \mathrm{m}, \mathrm{H} 6 \alpha), 1.43(2 \mathrm{H}, \mathrm{m}, \mathrm{H} 5 \alpha+\mathrm{H} 15 \alpha), 1.30(1 \mathrm{H}, \mathrm{m}, \mathrm{H} 1 \alpha), 1.24(3 \mathrm{H}, \mathrm{s}, \mathrm{H} 28), 1.22(3 \mathrm{H}, \mathrm{m}$, H21), $1.20+1.18\left(6 \mathrm{H}, 2 \mathrm{~d}, \mathrm{~J}=6.8 \mathrm{~Hz}, \mathrm{H} 3^{\prime}+\mathrm{H}^{\prime}\right), 1.14(1 \mathrm{H}, \mathrm{m}, \mathrm{H} 19 \beta), 1.02$ (3H, s, H18), 0.95 $(1 \mathrm{H}, \mathrm{m}, \mathrm{H} 6 \beta), 0.78(3 \mathrm{H}, \mathrm{s}, \mathrm{H} 30) ;{ }^{13} \mathrm{C} \mathrm{NMR}\left(75.4 \mathrm{MHz}, \mathrm{CDCl}_{3}\right): \delta(\mathrm{ppm}) 210.7(\mathrm{C} 11), 172.6$ (C31), 172.0 (C1'), 137.6 (C32), 129.3 (C35), 128.3 (C34+C36), 126.8 (C33+C37), 75.9 (C16), 62.6 (C3), 56.9 (C17), 51.6 (C12), 49.9 (C5), 48.5 (C20), 47.4 (C14), 45.9 (C15+C13), 41.5 (C8), 40.0 (C2'), 38.7 (C29), 38.5 (C10), 34.0 (C9), 33.5 (C4), 31.4 ( $\left.\mathrm{N}_{\mathrm{B}} \mathrm{Me}\right), 30.5$ (C2+C19), 27.9 (C1), 24.6 (C7), 21.1+20.8 (C3'+C4'), 19.9 (C28), 18.1 (C18+C21), 17.7 (C6), 11.3 (C30); MS (ESI, m/z) : $591.3(\mathrm{M}+\mathrm{H})$; HRMS (ESI, m/z) : calcd for $\mathrm{C}_{31} \mathrm{H}_{49} \mathrm{~N}_{2} \mathrm{O}_{3} \mathrm{~S}: 591.6320$, found : 591.3627. 


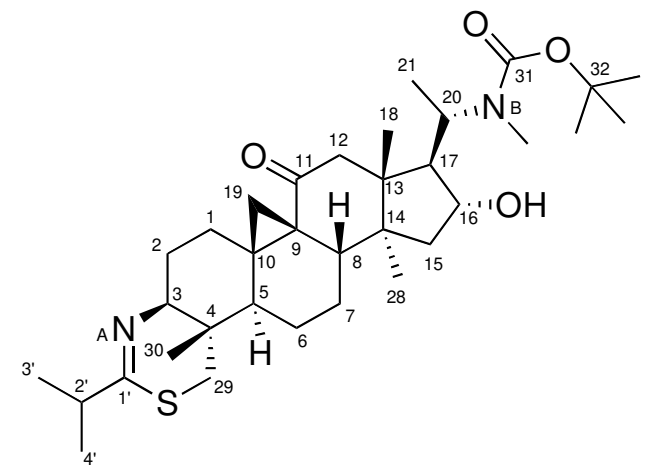

$17 \mathrm{mg}(0.035 \mathrm{mmol}, 1$ eq.) of $\mathbf{3 4}, 22 \mu \mathrm{L}(0.105 \mathrm{mmol}, 3 \mathrm{eq}$.) of di-tert-butyl dicarbonate and $1 \mathrm{~mL}$ of THF were used by following similar procedure described for the preparation of 35a to give the title compound as an amorphous white solid (15 mg, yield: $83 \%)$. IR $\left(\mathrm{cm}^{-1}\right): 3457$, 2965, 2931, 2869, 1668, 1633, 1455, 1260, $1140 .{ }^{1} \mathrm{H}$ NMR $\left(500 \mathrm{MHz}, \mathrm{CDCl}_{3}\right): \delta(\mathrm{ppm}) 4.46$ $(1 \mathrm{H}, \mathrm{m}, \mathrm{H} 20), 4.26(1 \mathrm{H}, \mathrm{m}, \mathrm{H} 16 \beta), 2.88(1 \mathrm{H}, \mathrm{dd}, \mathrm{J}=12.8 \mathrm{~Hz}, \mathrm{~J}=3.5 \mathrm{~Hz}, \mathrm{H} 3 \alpha), 2.84(1 \mathrm{H}, \mathrm{d}$, $\left.\mathrm{J}_{\mathrm{gem}}=11.6 \mathrm{~Hz}, \mathrm{H} 29 \mathrm{~b}\right), 2.76\left(3 \mathrm{H}, \mathrm{s}, \mathrm{N}_{\mathrm{B}} \mathrm{Me}\right), 2.74\left(1 \mathrm{H}, \mathrm{d}, \mathrm{J}_{\mathrm{gem}}=11.6 \mathrm{~Hz}, \mathrm{H} 29 \mathrm{a}\right), 2.68(1 \mathrm{H}, \mathrm{m}$, $\left.\mathrm{H} 2^{\prime}\right), 2.55\left(1 \mathrm{H}, \mathrm{d}, \mathrm{J}_{\text {gem }}=17.1 \mathrm{~Hz}, \mathrm{H} 12 \alpha\right), 2.47(1 \mathrm{H}, \mathrm{m}, \mathrm{H} 1 \beta), 2.36\left(1 \mathrm{H}, \mathrm{d}, \mathrm{J}_{\text {gem }}=17.1 \mathrm{~Hz}\right.$,

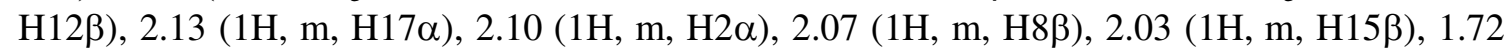
$(1 \mathrm{H}, \mathrm{m}, \mathrm{H} 2 \beta), 1.65\left(1 \mathrm{H}, \mathrm{d}, \mathrm{J}_{\text {gem }}=3.7 \mathrm{~Hz}, \mathrm{H} 19 \alpha\right), 1.57(2 \mathrm{H}, \mathrm{m}, \mathrm{H} 7), 1.55(1 \mathrm{H}, \mathrm{m}, \mathrm{H} 6 \alpha), 1.43$ $(2 \mathrm{H}, \mathrm{m}, \mathrm{H} 5 \alpha+\mathrm{H} 15 \alpha), 1.32(10 \mathrm{H}, \mathrm{m}, \mathrm{H} 1 \alpha+\mathrm{Boc}), 1.21(3 \mathrm{H}, \mathrm{s}, \mathrm{H} 28), 1.19+1.18(6 \mathrm{H}, 2 \mathrm{~d}, \mathrm{~J}=$ $6.7 \mathrm{~Hz}, \mathrm{H} 3$ ' $+\mathrm{H} 4$ ') $, 1.12(1 \mathrm{H}, \mathrm{m}, \mathrm{H} 19 \beta), 1.10(3 \mathrm{H}, \mathrm{m}, \mathrm{H} 21), 1.02(1 \mathrm{H}, \mathrm{m}, \mathrm{H} 6 \beta), 0.93$ (3H, s, $\mathrm{H} 18), 0.77$ (3H, s, H30) ; ${ }^{13} \mathrm{C}$ NMR (75.4 MHz, $\left.\mathrm{CDCl}_{3}\right): \delta(\mathrm{ppm}) 210.5(\mathrm{C} 11), 171.6(\mathrm{C} 1$ '), 156.7 (C31), 79.7 (C32), 76.0 (C16), 62.6 (C3), 57.8 (C17), 51.6 (C12), 50.8 (C20), 49.8 (C5), 47.3 (C14), 45.5 (C13), 45.2 (C15), 41.5 (C8), 40.1 (C2'), 38.7 (C29), 38.5 (C10), 34.1 (C9), 33.5 (C4), 30.5 (C19), 30.2(C2), $28.5\left(\mathrm{~N}_{\mathrm{B}} \mathrm{Me}+\mathrm{N}_{\mathrm{B}} \mathrm{Boc}\right), 27.9$ (C1), 24.5 (C7), 21.1 (C28), 20.8+19.9 (C3'+C4'), 18.2 (C21), 18.1 (C6), 17.9 (C18), 11.3 (C30); MS (ESI, m/z) : $587.4(\mathrm{M}+\mathrm{H})$; HRMS (ESI, m/z) : calcd for $\mathrm{C}_{34} \mathrm{H}_{55} \mathrm{~N}_{2} \mathrm{O}_{4} \mathrm{~S}: 587.3883$, found : 587.3884 . 
20S-[benzyl(methyl)amino]-16 $\alpha$-hydroxy-4 $\beta, 14 \alpha$-dimethyl-9,19-cyclo-\{2'-isopropyl5',6'-dihydro-4' $\alpha H-\left[1^{\prime}, 3\right.$ ']thiazino[4',5':3,4]\}-5 $\alpha, 9 \beta$-pregnan-11-one (36a).

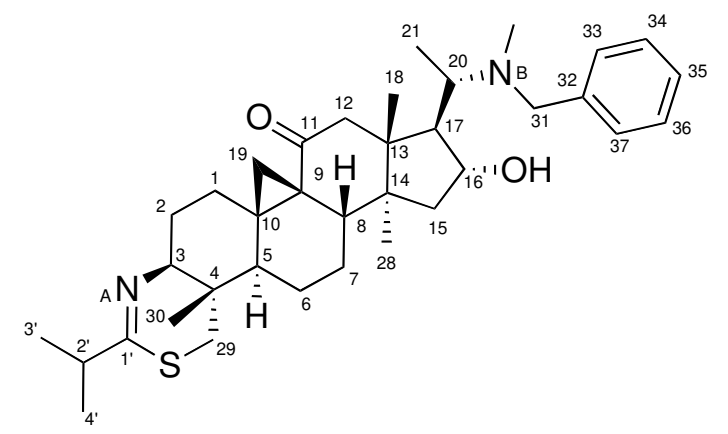

To $13 \mathrm{mg}(0.0267 \mathrm{mmol}, 1$ eq. $)$ of $\mathbf{3 4}$ dissolved in $1 \mathrm{~mL}$ of acetonitrile were added $4.1 \mu \mathrm{L}$ ( $0.035 \mathrm{mmol}, 1.3$ eq.) of benzyl bromide and $11 \mathrm{mg}(0.08 \mathrm{mmol}, 3$ eq.) of potassium carbonate. The mixture was stirred at RT for $16 \mathrm{~h}$, diluted with $5 \mathrm{~mL}$ of a saturated sodium hydrogen carbonate solution then extracted with $3 \times 5 \mathrm{~mL}$ of DCM. Combined organic layers were washed with brine, dried over magnesium sulfate and concentrated under vacuum. The crude product was purified by silica gel column chromatography (gradient elution: $\mathrm{DCM} / \mathrm{MeOH} / \mathrm{NH}_{4} \mathrm{OH}$ : from $99 / 0 / 1$ to $96 / 3 / 1$ ) to afford the title compound as an amorphous white solid (14 mg, yield: $93 \%)$. IR $\left(\mathrm{cm}^{-1}\right)$ : 3324, 2962, 2929, 2867, 1666, 1633, 1452, 1259, 1223, 1147, 1079, 1017 ; ${ }^{1} \mathrm{H}$ NMR (500 $\left.\mathrm{MHz}, \mathrm{CD}_{3} \mathrm{CN}, 333 \mathrm{~K}\right): \delta(\mathrm{ppm}) 7.30(5 \mathrm{H}, \mathrm{m}, \mathrm{H} 33+\mathrm{H} 34+\mathrm{H} 35+\mathrm{H} 36+\mathrm{H} 37), 3.99(1 \mathrm{H}, \mathrm{m}$, $\mathrm{H} 16 \beta), 3.72\left(1 \mathrm{H}, \mathrm{d}, \mathrm{J}_{\mathrm{gem}}=12.8 \mathrm{~Hz}, \mathrm{H} 31\right), 3.59\left(1 \mathrm{H}, \mathrm{d}, \mathrm{J}_{\mathrm{gem}}=12.8 \mathrm{~Hz}, \mathrm{H} 31\right), 2.86(2 \mathrm{H}$, m, H20+H29b), $2.84(1 \mathrm{H}, \mathrm{m}, \mathrm{H} 3 \alpha), 2.75\left(1 \mathrm{H}, \mathrm{d}, \mathrm{J}_{\mathrm{gem}}=11.6 \mathrm{~Hz}, \mathrm{H} 29 \mathrm{a}\right), 2.53(1 \mathrm{H}, \mathrm{d}$, $\left.\mathrm{J}_{\mathrm{gem}}=17.1 \mathrm{~Hz}, \mathrm{H} 12 \alpha\right), 2.48\left(1 \mathrm{H}, \mathrm{m}, \mathrm{H} 2^{\prime}\right), 2.35\left(1 \mathrm{H}, \mathrm{dt}, \mathrm{J}_{\mathrm{gem}}=13.6 \mathrm{~Hz}, \mathrm{~J}_{1 \beta-2}=3.2 \mathrm{~Hz}\right.$, $\mathrm{H} 1 \beta), 2.23\left(1 \mathrm{H}, \mathrm{d}, \mathrm{J}_{\text {gem }}=17.1 \mathrm{~Hz}, \mathrm{H} 12 \beta\right), 2.21\left(3 \mathrm{H}, \mathrm{s}, \mathrm{N}_{\mathrm{BMe}}\right), 2.12(1 \mathrm{H}, \mathrm{m}, \mathrm{H} 17 \alpha), 2.08$ (1H, m, H8 $\beta), 1.97(1 \mathrm{H}, \mathrm{m}, \mathrm{H} 15 \beta), 1.95(1 \mathrm{H}, \mathrm{m}, \mathrm{H} 2 \alpha), 1.63(1 \mathrm{H}, \mathrm{m}, \mathrm{H} 2 \beta), 1.53(1 \mathrm{H}, \mathrm{m}$, H7 $\beta), 1.51(1 \mathrm{H}, \mathrm{m}, \mathrm{H} 6 \alpha), 1.50\left(1 \mathrm{H}, \mathrm{d}, \mathrm{J}_{\text {gem }}=4.0 \mathrm{~Hz}, \mathrm{H} 19 \alpha\right), 1.47(1 \mathrm{H}, \mathrm{m}, \mathrm{H} 5 \alpha), 1.39$ $(1 \mathrm{H}, \mathrm{m}, \mathrm{H} 15 \alpha), 1.33(1 \mathrm{H}, \mathrm{m}, \mathrm{H} 7 \alpha), 1.31$ (1H, m, H1 $\alpha), 1.18(3 \mathrm{H}, \mathrm{s}, \mathrm{H} 28), 1.13(1 \mathrm{H}, \mathrm{m}$,

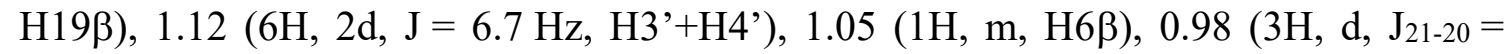
$6.4 \mathrm{~Hz}, \mathrm{H} 21), 0.75(3 \mathrm{H}, \mathrm{s}, \mathrm{H} 18), 0.73(3 \mathrm{H}, \mathrm{s}, \mathrm{H} 30) ;{ }^{13} \mathrm{C} \mathrm{NMR}\left(75.4 \mathrm{MHz}, \mathrm{CD}_{3} \mathrm{CN}\right): \delta(\mathrm{ppm})$ 210.8 (C11), 165.9 (C1'), 138.7 (C32), 129.1 (C33+C37), 128.5 (C34+C36), 127.3 (C35), 77.6 (C16), 62.7 (C3), 60.3 (C20), 59.4 (C31), 55.8 (C17), 51.4 (C12), 49.7 (C5), 47.2 (C14), 44.7 (C13), 42.8 (C15), 41.3 (C8), 40.2 (C2'), 38.7 (C29), 38.4 (C10), 35.3 (NBMe), 34.4 (C9), 33.4 (C4), 30.5 (C2), 30.3 (C19), 28.0 (C1), 24.4 (C7), 21.0+20.8 (C3'+C4'), 20.6 (C28), 18.0 (C6), 17.7 (C18), 11.3 (C30), 10.7 (C21) ; MS (ESI, m/z) : $577.4(\mathrm{M}+\mathrm{H}), 487.3(\mathrm{M}-\mathrm{Bn}+\mathrm{H})$; HRMS (ESI, m/z) : calcd for $\mathrm{C}_{36} \mathrm{H}_{53} \mathrm{~N}_{2} \mathrm{O}_{2} \mathrm{~S}: 577.3828$, found : 577.3835 . 


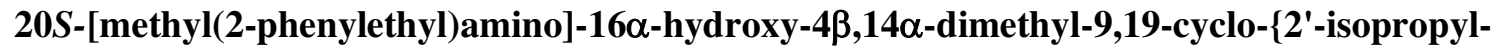
5',6'-dihydro-4' $\alpha H$-[1',3']thiazino $\left[4^{\prime}, 5\right.$ ':3,4]\}-5 $\alpha, 9 \beta$-pregnan-11-one (36b)

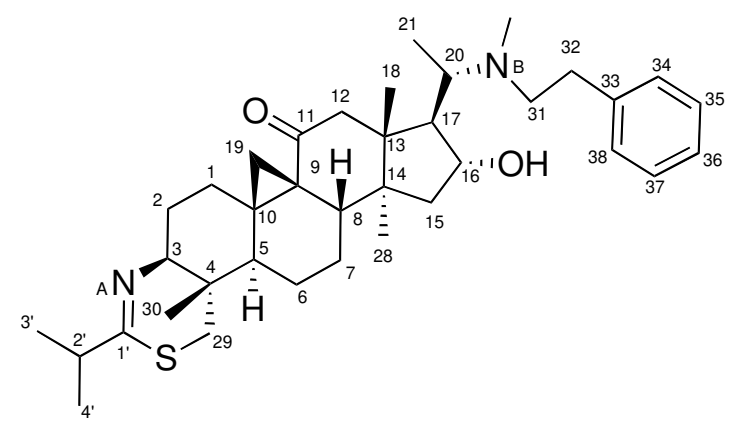

$18.5 \mathrm{mg}$ ( $0.038 \mathrm{mmol}, 1$ eq.) of $\mathbf{3 4}, 7 \mu \mathrm{L}$ ( $0.05 \mathrm{mmol}, 1.3 \mathrm{eq}$.) of (2-bromoethyl)benzene, $16 \mathrm{mg}(0.114 \mathrm{mmol}, 3 \mathrm{eq}$. $)$ of potassium carbonate and $1 \mathrm{~mL}$ of acetonitrile were used by following similar procedure described for the preparation of 36a to give the title compound as an amorphous white solid (19 mg, yield: $84 \%$ ). For this reaction, the mixture was refluxed for $12 \mathrm{~h}$ to give the desired product. IR $\left(\mathrm{cm}^{-1}\right): 3381,3025(\mathrm{H}), 2961,2930,2865,1666,1632,1496$, $1454,1260,1224,1154,1117,1016 ;{ }^{1} \mathrm{H}$ NMR $\left(500 \mathrm{MHz}, \mathrm{CD}_{3} \mathrm{CN}, 333 \mathrm{~K}\right): \delta(\mathrm{ppm}) 7.27(5 \mathrm{H}, \mathrm{m}$, $\mathrm{H} 34+\mathrm{H} 35+\mathrm{H} 36+\mathrm{H} 37+\mathrm{H} 38), 4.01(1 \mathrm{H}, \mathrm{m}, \mathrm{H} 16 \beta), 2.91\left(1 \mathrm{H}, \mathrm{d}, \mathrm{J}_{\mathrm{gem}}=11.7 \mathrm{~Hz}, \mathrm{H} 29 \mathrm{~b}\right), 2.86(1 \mathrm{H}$, m, H20), $2.82(4 \mathrm{H}, \mathrm{m}, \mathrm{H} 31+\mathrm{H} 32), 2.79(2 \mathrm{H}, \mathrm{m}, \mathrm{H} 3 \alpha+\mathrm{H} 29 \mathrm{a}), 2.56\left(1 \mathrm{H}, \mathrm{d}, \mathrm{J}_{\mathrm{gem}}=17.0 \mathrm{~Hz}\right.$, $\mathrm{H} 12 \alpha), 2.50\left(1 \mathrm{H}, \mathrm{m}, \mathrm{H} 2^{\prime}\right), 2.39\left(1 \mathrm{H}, \mathrm{dt}, \mathrm{J}_{\mathrm{gem}}=13.2 \mathrm{~Hz}, \mathrm{~J}_{1 \beta-2}=3.4 \mathrm{~Hz}, \mathrm{H} 1 \beta\right), 2.29(3 \mathrm{H}, \mathrm{s}$, $\left.\mathrm{N}_{\mathrm{B}} \mathrm{Me}\right), 2.25\left(1 \mathrm{H}, \mathrm{d}, \mathrm{J}_{\mathrm{gem}}=17.0 \mathrm{~Hz}, \mathrm{H} 12 \beta\right), 2.13(1 \mathrm{H}, \mathrm{m}, \mathrm{H} 8 \beta), 2.05(1 \mathrm{H}, \mathrm{m}, \mathrm{H} 17 \alpha), 2.01(1 \mathrm{H}$, $\mathrm{m}, \mathrm{H} 15 \beta), 1.97(1 \mathrm{H}, \mathrm{m}, \mathrm{H} 2 \alpha), 1.68(1 \mathrm{H}, \mathrm{m}, \mathrm{H} 2 \beta), 1.57(1 \mathrm{H}, \mathrm{m}, \mathrm{H} 7 \beta), 1.54\left(1 \mathrm{H}, \mathrm{d}, \mathrm{J}_{\mathrm{gem}}=4.0 \mathrm{~Hz}\right.$,

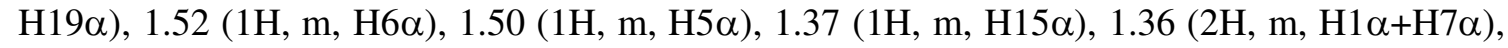
$1.21(3 \mathrm{H}, \mathrm{s}, \mathrm{H} 28), 1.18(1 \mathrm{H}, \mathrm{m}, \mathrm{H} 19 \beta), 1.17+1.16\left(6 \mathrm{H}, 2 \mathrm{~d}, \mathrm{~J}=6.8 \mathrm{~Hz}, \mathrm{H} 3\right.$ ' $\left.+\mathrm{H} 4{ }^{\prime}\right), 1.09$ (1H, m, H6ß), $0.95\left(3 \mathrm{H}, \mathrm{d}, \mathrm{J}_{21-20}=6.6 \mathrm{~Hz}, \mathrm{H} 21\right), 0.88(3 \mathrm{H}, \mathrm{s}, \mathrm{H} 18), 0.79(3 \mathrm{H}, \mathrm{s}, \mathrm{H} 30) ;{ }^{13} \mathrm{C}$ NMR $(75.4$ $\left.\mathrm{MHz}, \mathrm{CD}_{3} \mathrm{CN}\right): \delta(\mathrm{ppm}) 211.3(\mathrm{C} 11), 165.3(\mathrm{C} 1$ '), $141.8(\mathrm{C} 33), 129.8(\mathrm{C} 34+\mathrm{C} 38), 129.5$ (C35+C37), 127.1 (C36), 78.9 (C16), 64.1 (C20), 62.5 (C3), 57.6 (C17), 57.3 (C31), 52.7 (C12), 50.6 (C5), 48.4 (C14), 46.1 (C13), 44.2 (C15), 42.6 (C8), 40.7 (C2'), 40.0 (C29), 38.9 (C10), 35.9 (C32), 35.6 ( $\mathrm{N}_{\mathrm{B}} \mathrm{Me}$ ), 34.4 (C9), 33.4 (C4), 31.8 (C2), 30.6 (C19), 29.3 (C1), 25.4 (C7), 21.5+21.4 (C3'+C4'), 21.0 (C28), 19.1 (C6), 18.5 (C18), 11.9 (C30), 11.6 (C21); MS (ESI, m/z) : $591.4(\mathrm{M}+\mathrm{H})$; HRMS (ESI, m/z) : calcd for $\mathrm{C}_{37} \mathrm{H}_{55} \mathrm{~N}_{2} \mathrm{O}_{2} \mathrm{~S}:$ : 591.3984, found : 591.3972. 


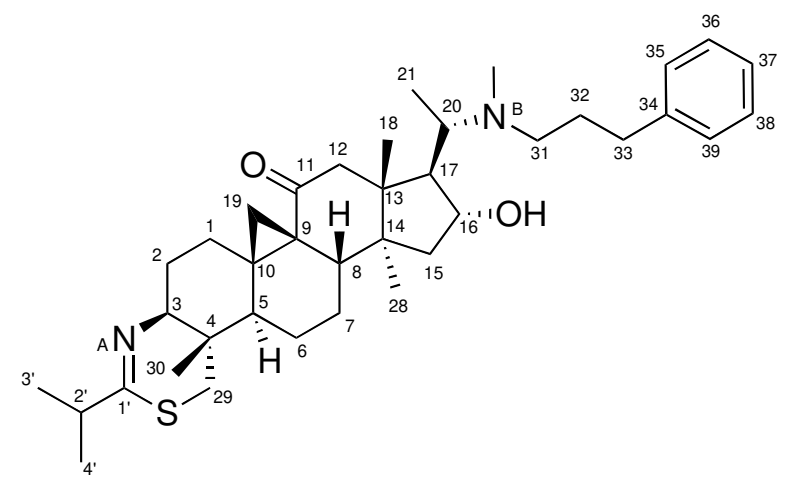

$15 \mathrm{mg}(0.031 \mathrm{mmol}, 1$ eq. $)$ of $\mathbf{3 4}, 13 \mathrm{mg}(0.092 \mathrm{mmol}, 3$ eq. $)$ of potassium carbonate, 6 $\mu \mathrm{L}(0.043 \mathrm{mmol}, 1.3$ eq.) of 1 -bromo-3-phenylpropane and $0.5 \mathrm{~mL}$ of DMF were used by following similar procedure described for the preparation of 36a to give the title compound as an amorphous white solid (14 mg, yield: $72 \%)$. IR $\left(\mathrm{cm}^{-1}\right): 3381,3025,2962,2930,2863,1666$, 1632, 1454, 1259, 1224, 1156, $1018 ;{ }^{1} \mathrm{H}$ NMR $\left(500 \mathrm{MHz}, \mathrm{CD}_{3} \mathrm{CN}\right): \delta(\mathrm{ppm}) 7.21(2 \mathrm{H}, \mathrm{m}$, $\mathrm{H} 36+\mathrm{H} 38), 7.11(3 \mathrm{H}, \mathrm{m}, \mathrm{H} 35+\mathrm{H} 37+\mathrm{H} 39), 4.04(1 \mathrm{H}, \mathrm{m}, \mathrm{H} 16 \beta), 2.78(1 \mathrm{H}, \mathrm{dd}, \mathrm{J}=12.8 \mathrm{~Hz}, \mathrm{~J}=$ $4.0 \mathrm{~Hz}, \mathrm{H} 3 \alpha), 2.76(1 \mathrm{H}, \mathrm{d}, \mathrm{J}=11.9, \mathrm{H} 29 \mathrm{~b}), 2.66\left(1 \mathrm{H}, \mathrm{d}, \mathrm{J}_{\text {gem }}=11.9 \mathrm{~Hz}, \mathrm{H} 29 \mathrm{a}\right), 2.65(1 \mathrm{H}, \mathrm{m}$, H20), $2.56(2 \mathrm{H}, \mathrm{t}, \mathrm{J}=8.1 \mathrm{~Hz}, \mathrm{H} 33), 2.51(1 \mathrm{H}$, septuplet, J = 7.0 Hz, H2'), $2.44(2 \mathrm{H}, \mathrm{m}, \mathrm{H} 31)$, $2.43\left(1 \mathrm{H}, \mathrm{d}, \mathrm{J}_{\mathrm{gem}}=17.1 \mathrm{~Hz}, \mathrm{H} 12 \alpha\right), 2.38\left(1 \mathrm{H}, \mathrm{dt}, \mathrm{J}_{\mathrm{gem}}=13.4 \mathrm{~Hz}, \mathrm{~J}=4.0 \mathrm{~Hz}, \mathrm{H} 1 \beta\right), 2.24(1 \mathrm{H}, \mathrm{d}$, $\left.\mathrm{J}_{\mathrm{gem}}=17.1 \mathrm{~Hz}, \mathrm{H} 12 \beta\right), 2.09\left(3 \mathrm{H}, \mathrm{s}, \mathrm{N}_{\mathrm{B}} \mathrm{Me}\right), 2.01(2 \mathrm{H}, \mathrm{m}, \mathrm{H} 2 \alpha+\mathrm{H} 8 \beta), 1.96(1 \mathrm{H}, \mathrm{m}$, $\mathrm{H} 15 \beta+\mathrm{H} 17 \alpha), 1.75(2 \mathrm{H}, \mathrm{m}, \mathrm{H} 32), 1.62(1 \mathrm{H}, \mathrm{m}, \mathrm{H} 2 \beta), 1.57\left(1 \mathrm{H}, \mathrm{d}, \mathrm{J}_{\text {gem }}=4.0 \mathrm{~Hz}, \mathrm{H} 19 \alpha\right), 1.53$ $(1 \mathrm{H}, \mathrm{m}, \mathrm{H} 7 \beta), 1.49(1 \mathrm{H}, \mathrm{m}, \mathrm{H} 6 \alpha), 1.42(1 \mathrm{H}, \mathrm{d}, \mathrm{J}=14.0 \mathrm{~Hz}, \mathrm{~J}=2.7 \mathrm{~Hz}, \mathrm{H} 15 \alpha), 1.35(1 \mathrm{H}, \mathrm{d}, \mathrm{J}=$ $12.5 \mathrm{~Hz}, \mathrm{~J}=3.7 \mathrm{~Hz}, \mathrm{H} 5 \alpha), 1.24(2 \mathrm{H}, \mathrm{m}, \mathrm{H} 1 \alpha+\mathrm{H} 7 \alpha), 1.14(3 \mathrm{H}, \mathrm{s}, \mathrm{H} 28), 1.11+1.10(6 \mathrm{H}, 2 \mathrm{~d}, \mathrm{~J}=$

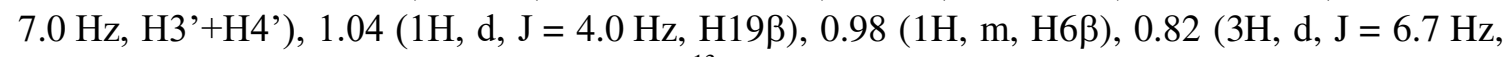
$\mathrm{H} 21), 0.78$ (3H, s, H18), 0.69 (3H, s, H30); ${ }^{13} \mathrm{C}$ NMR (75.4 MHz, $\left.\mathrm{CD}_{3} \mathrm{CN}\right): \delta(\mathrm{ppm}) 211.7(\mathrm{C} 11)$, 165.8 (C1'), 141.9 (C34), 128.4 (C35+C36+C38+C39), 125.9 (C37), 78.2 (C16), 62.7 (C3), 60.4 (C20), 56.2 (C31), 55.7 (C17), 51.4 (C12), 49.8 (C5), 47.2 (C14), 44.6 (C13), 42.8 (C15), 41.4 (C8), 40.3 (C2'), 38.8 (C29), 38.5 (C10), 34.5 ( $\left.\mathrm{N}_{\mathrm{B}} \mathrm{Me}+\mathrm{C} 9\right), 33.7$ (C33), 33.4 (C4), 30.6 (C2), 30.3 (C19), 30.2 (C32), 28.0 (C1), 24.5 (C7), 21.0+20.8 (C3'+C4'), 20.7 (C28), 18.1 (C6), 17.9 (C18), 11.3 (C30), 10.9 (C21); MS (ESI, m/z) : $605.3(\mathrm{M}+\mathrm{H})$; HRMS (ESI, m/z) : calcd for $\mathrm{C}_{38} \mathrm{H}_{57} \mathrm{~N}_{2} \mathrm{O}_{2} \mathrm{~S}: 605.4141$, found : 605.4152 . 


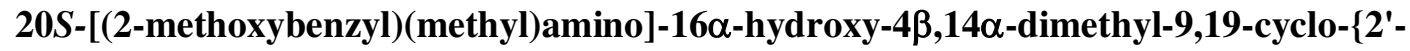
isopropyl-5',6'-dihydro-4' $\alpha H$-[1',3']thiazino $\left.\left[4^{\prime}, 5^{\prime}: 3,4\right]\right\}-5 \alpha, 9 \beta$-pregnan-11-one (36d)

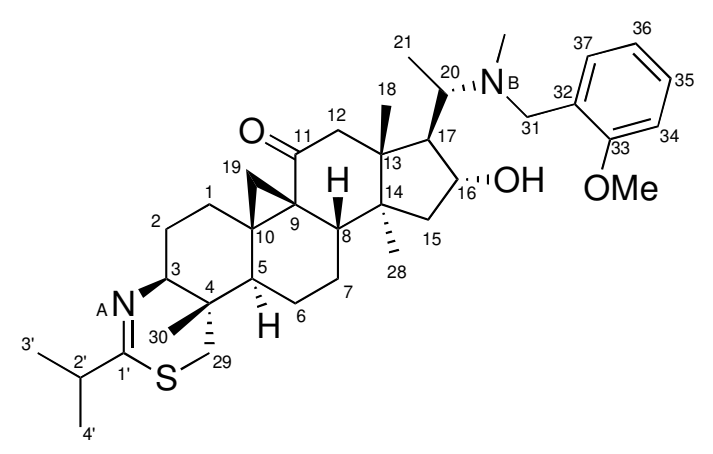

$15 \mathrm{mg}$ ( $0.031 \mathrm{mmol}, 1$ eq.) of $\mathbf{3 4}, 6 \mu \mathrm{L}(0.037 \mathrm{mmol}, 1.2$ eq.) of 2-methoxybenzyl chloride, $6.5 \mathrm{mg}(0.046 \mathrm{mmol}, 1.5$ eq. $)$ of potassium carbonate and $1 \mathrm{~mL}$ of DMF were used by following similar procedure described for the preparation of 36a to give the title compound as an amorphous white solid (13 mg, yield: $69 \%$ ). For this reaction, the mixture was stirred at $130{ }^{\circ} \mathrm{C}$ for $30 \mathrm{~min}$ to give the desired product. IR $\left(\mathrm{cm}^{-1}\right): 3324,2962,2929,2867,1666,1633,1452$, 1259, 1223, 1147, 1079, $1017 ;{ }^{1} \mathrm{H}$ NMR (500 MHz, CD $\left.{ }_{3} \mathrm{CN}\right): \delta(\mathrm{ppm}) 7.27(1 \mathrm{H}, \mathrm{m}, \mathrm{H} 37), 7.19$ (1H, m, H35), 6.92 (1H, m, H36), 6.89 (1H, m, H34), 3.85 (3H, s, OMe), 3.73 (1H, m, H16 $)$, $3.63(2 \mathrm{H}, \mathrm{bs}, \mathrm{H} 31), 2.86(1 \mathrm{H}, \mathrm{m}, \mathrm{H} 3 \alpha), 2.84(1 \mathrm{H}, \mathrm{d}, \mathrm{J}=11.3, \mathrm{H} 29 \mathrm{a}), 2.80(1 \mathrm{H}, \mathrm{m}, \mathrm{H} 20), 2.74$ $\left(1 \mathrm{H}, \mathrm{d}, \mathrm{J}_{\mathrm{gem}}=11.3 \mathrm{~Hz}, \mathrm{H} 29 \mathrm{~b}\right), 2.60\left(1 \mathrm{H}, \mathrm{sept}, \mathrm{J}=6.8 \mathrm{~Hz}, \mathrm{H} 2^{\prime}\right), 2.51\left(1 \mathrm{H}, \mathrm{d}, \mathrm{J}_{\text {gem }}=16.4 \mathrm{~Hz}\right.$, $\mathrm{H} 12 \alpha), 2.45\left(1 \mathrm{H}, \mathrm{dm}, \mathrm{J}_{\mathrm{gem}}=13.8 \mathrm{~Hz}, \mathrm{H} 1 \beta\right), 2.32\left(1 \mathrm{H}, \mathrm{d}, \mathrm{J}_{\mathrm{gem}}=16.4 \mathrm{~Hz}, \mathrm{H} 12 \beta\right), 2.18(3 \mathrm{H}, \mathrm{s}$, $\left.\mathrm{N}_{\mathrm{B}} \mathrm{Me}\right), 2.05(2 \mathrm{H}, \mathrm{m}, \mathrm{H} 2 \alpha+\mathrm{H} 8 \beta), 1.99(1 \mathrm{H}, \mathrm{m}, \mathrm{H} 17 \alpha), 1.93(1 \mathrm{H}, \mathrm{m}, \mathrm{H} 15 \beta), 1.69$ (1H, m, H2 $\beta$ ), $1.64\left(1 \mathrm{H}, \mathrm{d}, \mathrm{J}_{\mathrm{gem}}=3.8 \mathrm{~Hz}, \mathrm{H} 19 \alpha\right), 1.53(2 \mathrm{H}, \mathrm{m}, \mathrm{H} 6 \alpha+\mathrm{H} 7 \beta), 1.45(2 \mathrm{H}, \mathrm{m}, \mathrm{H} 5 \alpha+\mathrm{H} 15 \alpha), 1.34$ $(1 \mathrm{H}, \mathrm{m}, \mathrm{H} 1 \alpha), 1.31(1 \mathrm{H}, \mathrm{m}, \mathrm{H} 7 \alpha), 1.20+1.18\left(6 \mathrm{H}, 2 \mathrm{~d}, \mathrm{~J}=6.8 \mathrm{~Hz}, \mathrm{H} 3^{\prime}+\mathrm{H}^{\prime}{ }^{\prime}\right), 1.18(3 \mathrm{H}, \mathrm{s}, \mathrm{H} 28)$, $1.10(1 \mathrm{H}, \mathrm{d}, \mathrm{J}=3.8 \mathrm{~Hz}, \mathrm{H} 19 \beta), 0.97$ (1H, m, H6ß), 0.96 (3H, m, H21), 0.77 (3H, s, H30), 0.75 $(3 \mathrm{H}, \mathrm{s}, \mathrm{H} 18) ;{ }^{13} \mathrm{C}$ NMR $\left(75.4 \mathrm{MHz}, \mathrm{CD}_{3} \mathrm{CN}\right): \delta(\mathrm{ppm}) 211.0(\mathrm{C} 11), 165.8(\mathrm{C} 1$ '), $158.2(\mathrm{C} 33)$, 131.2 (C35), 128.7 (C33), 126.8 (C32), 120.1 (C36), 110.3 (C34), 77.9 (C16), 62.7 (C3), 58.7 (C20), 55.6 (C31+C17), 55.1 (OMe), 51.4 (C12), 49.8 (C5), 47.1 (C14), 44.5 (C13), 42.8 (C15), 41.3 (C8), 40.3 (C2'), 38.8 (C29), 38.4 (C10), 34.5 ( $\left.\mathrm{N}_{\mathrm{B}} \mathrm{Me}+\mathrm{C} 9\right), 33.4$ (C4), 30.6 (C2), 30.2 (C19), 28.0 (C1), 24.4 (C7), 21.0+20.8 (C3'+C4'), 20.6 (C28), 18.0 (C6), 17.7 (C18), 11.4 (C30), 10.8 (C21); MS (ESI, m/z) : $607.3(\mathrm{M}+\mathrm{H})$; HRMS (ESI, m/z) : calcd for $\mathrm{C}_{37} \mathrm{H}_{55} \mathrm{~N}_{2} \mathrm{O}_{3} \mathrm{~S}$ : 606.3933, found : 607.3923 . 


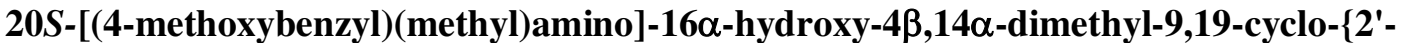 isopropyl-5',6'-dihydro-4' $\alpha H$-[1',3']thiazino $\left.\left[4^{\prime}, 5^{\prime}: 3,4\right]\right\}-5 \alpha, 9 \beta-p r e g n a n-11-o n e(36 f)$}

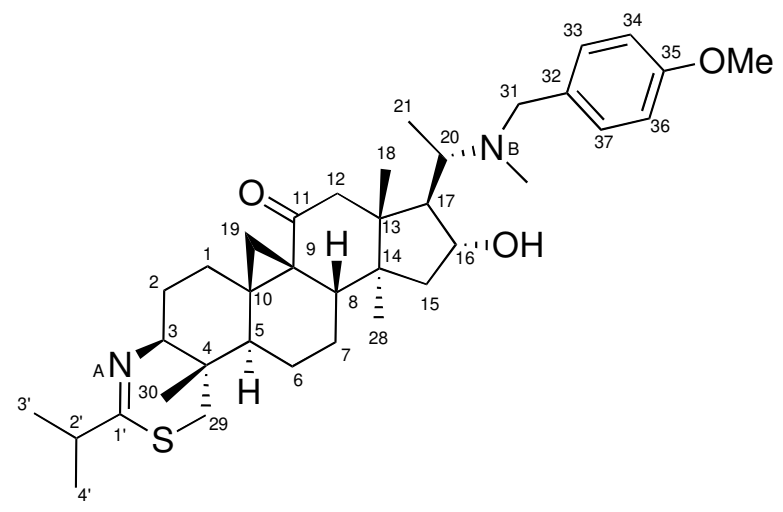

$16 \mathrm{mg}(0.033 \mathrm{mmol}, 1$ eq. $)$ of $\mathbf{3 4}, 14 \mathrm{mg}(0.099 \mathrm{mmol}, 3$ eq. $)$ of potassium carbonate, 6 $\mu \mathrm{L}(0.043 \mathrm{mmol}, 1.3$ eq.) of 4-methoxybenzyl chloride and $0.5 \mathrm{~mL}$ of DMF were used by following similar procedure described for the preparation of 36a to give the title compound as an amorphous white solid (14 mg, yield: $70 \%)$. IR $\left(\mathrm{cm}^{-1}\right): 3343,2964,2932,2867,1665,1611$, $1512,1462,1246,1173,1173,1035 ;{ }^{1} \mathrm{H}$ NMR $\left(500 \mathrm{MHz}, \mathrm{CD}_{3} \mathrm{CN}\right): \delta(\mathrm{ppm}) 7.21(2 \mathrm{H}, \mathrm{d}, \mathrm{J}=$ $8.5 \mathrm{~Hz}, \mathrm{H} 33+\mathrm{H} 37), 6.86(2 \mathrm{H}, \mathrm{d}, \mathrm{J}=8.5 \mathrm{~Hz}, \mathrm{H} 34+\mathrm{H} 36), 3.96$ (1H, bs, H16ß), 3.80 (3H, s, OMe), $3.58(2 \mathrm{H}, \mathrm{bs}, \mathrm{H} 31), 2.85(1 \mathrm{H}, \mathrm{dd}, \mathrm{J}=12.8 \mathrm{~Hz}, \mathrm{~J}=4.0 \mathrm{~Hz}, \mathrm{H} 3 \alpha), 2.82(1 \mathrm{H}, \mathrm{d}, \mathrm{J}=11.3, \mathrm{H} 29 \mathrm{~b})$, $2.81(1 \mathrm{H}, \mathrm{m}, \mathrm{H} 20), 2.73\left(1 \mathrm{H}, \mathrm{d}, \mathrm{J}_{\mathrm{gem}}=11.3 \mathrm{~Hz}, \mathrm{H} 29 \mathrm{a}\right), 2.59$ (1H, septuplet, J = 7.0 Hz, H2'), $2.51\left(1 \mathrm{H}, \mathrm{d}, \mathrm{J}_{\mathrm{gem}}=17.0 \mathrm{~Hz}, \mathrm{H} 12 \alpha\right), 2.44\left(1 \mathrm{H}, \mathrm{dt}, \mathrm{J}_{\mathrm{gem}}=13.4 \mathrm{~Hz}, \mathrm{~J}=4.0 \mathrm{~Hz}, \mathrm{H} 1 \beta\right), 2.31(1 \mathrm{H}, \mathrm{d}$, $\left.\mathrm{J}_{\text {gem }}=17.0 \mathrm{~Hz}, \mathrm{H} 12 \beta\right), 2.17\left(3 \mathrm{H}, \mathrm{s}, \mathrm{N}_{\mathrm{B}} \mathrm{Me}\right), 2.05(3 \mathrm{H}, \mathrm{m}, \mathrm{H} 2 \alpha+\mathrm{H} 8 \beta+\mathrm{H} 17 \alpha), 1.95(1 \mathrm{H}, \mathrm{m}$, $\mathrm{H} 15 \beta), 1.69(1 \mathrm{H}, \mathrm{m}, \mathrm{H} 2 \beta), 1.63\left(1 \mathrm{H}, \mathrm{d}, \mathrm{J}_{\mathrm{gem}}=4.0 \mathrm{~Hz}, \mathrm{H} 19 \alpha\right), 1.57(1 \mathrm{H}, \mathrm{m}, \mathrm{H} 7 \beta), 1.52(1 \mathrm{H}, \mathrm{m}$, $\mathrm{H} 6 \alpha), 1.46(1 \mathrm{H}, \mathrm{m}, \mathrm{H} 15 \alpha), 1.41(1 \mathrm{H}, \mathrm{m}, \mathrm{H} 5 \alpha), 1.30(2 \mathrm{H}, \mathrm{m}, \mathrm{H} 1 \alpha+\mathrm{H} 7 \alpha), 1.20(3 \mathrm{H}, \mathrm{s}, \mathrm{H} 28)$, $1.18+1.17(6 \mathrm{H}, 2 \mathrm{~d}, \mathrm{~J}=7.0 \mathrm{~Hz}, \mathrm{H} 3$ '+H4'), $1.09(1 \mathrm{H}, \mathrm{d}, \mathrm{J}=4.0 \mathrm{~Hz}, \mathrm{H} 19 \beta), 0.98(1 \mathrm{H}, \mathrm{m}, \mathrm{H} 6 \beta)$, $0.95(3 \mathrm{H}, \mathrm{m}, \mathrm{H} 21), 0.77(3 \mathrm{H}, \mathrm{s}, \mathrm{H} 30), 0.75(3 \mathrm{H}, \mathrm{s}, \mathrm{H} 18) ;{ }^{13} \mathrm{C}$ NMR $\left(75.4 \mathrm{MHz}, \mathrm{CD}_{3} \mathrm{CN}\right): \delta(\mathrm{ppm})$ 210.8 (C11), 166.1 (C1'), 158.9 (C35), 130.4 (C33+C37), 113.9 (C34+C36), 77.8 (C16), 62.7 (C3), 60.4 (C31), 58.9 (C20), 55.7 (C17), 55.2 (OMe), 51.5 (C12), 49.8 (C5), 47.2 (C14), 44.7 (C13), 42.7 (C15), 41.4 (C8), 40.2 (C2'), 38.7 (C29), 38.4 (C10), 34.5 ( $\left.\mathrm{N}_{\mathrm{B}} \mathrm{Me}\right), 34.4$ (C9), 33.4 (C4), 30.5 (C2), 30.3 (C19), 28.0 (C1), 24.4 (C7), 21.1+20.8 (C3'+C4'), 20.6 (C28), 18.0 (C6), 17.8 (C18), 11.3 (C30), 10.7 (C21); MS (ESI, m/z) : $745.4(\mathrm{M}+\mathrm{K}), 607.3$ (M+H); HRMS (ESI, $\mathrm{m} / \mathrm{z}$ ) : calcd for $\mathrm{C}_{37} \mathrm{H}_{55} \mathrm{~N}_{2} \mathrm{O}_{3} \mathrm{~S}: 607.3933$, found : 607.3925 . 


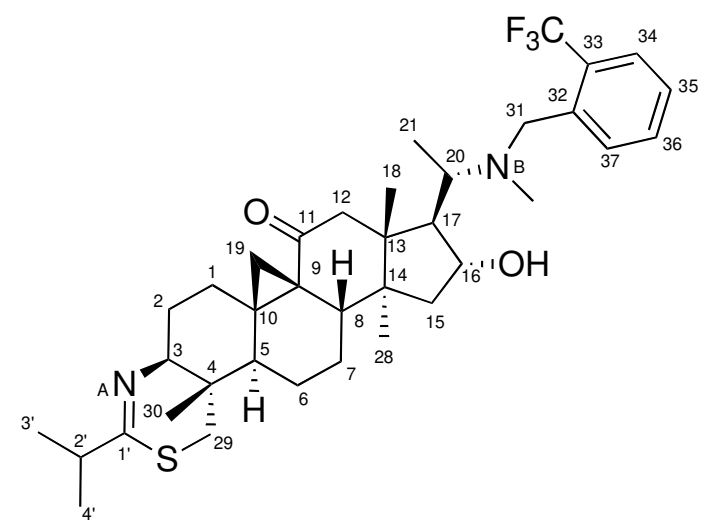

To $15 \mathrm{mg}(0,031 \mathrm{mmol}, 1$ eq.) of $\mathbf{3 4}$ dissolved in $0.5 \mathrm{~mL}$ of DMF were added $13 \mathrm{mg}$ $(0.093 \mathrm{mmol}, 3 \mathrm{eq}$.$) of potassium carbonate and 10 \mathrm{mg} \quad(0.040 \mathrm{mmol}, 1.3 \mathrm{eq}$.$) of$ 2-trifluoromethylbenzyl bromide. The mixture was stirred at $80{ }^{\circ} \mathrm{C}$ for $12 \mathrm{~h}$, diluted with $10 \mathrm{~mL}$ of a saturated sodium hydrogen carbonate solution and extracted with $3 \times 10 \mathrm{~mL}$ of $\mathrm{Et}_{2} \mathrm{O}$. Combined organic layers were washed with brine, dried over magnesium sulfate and concentrated under vacuum. The crude product was purified by silica gel column chromatography (gradient elution : Hept/Acetone/ $\mathrm{NH}_{4} \mathrm{OH}$ : from $60 / 40 / 1$ to $40 / 60 / 1$ ) to give the title compound as an amorphous white solid (11 mg, yield: $55 \%)$. IR $\left(\mathrm{cm}^{-1}\right): 3455,2960,2930$, 2867, 1666, 1633, 1454, 1311, 1257, 1160, 1120, $1037 ;{ }^{1} \mathrm{H}$ NMR (500 MHz, $\left.\mathrm{CD}_{3} \mathrm{CN}, 300 \mathrm{~K}\right)$ : $\delta(\mathrm{ppm}) 7.57(2 \mathrm{H}, \mathrm{m}, \mathrm{Har}), 7.47(1 \mathrm{H}, \mathrm{t}, \mathrm{J}=7.6 \mathrm{~Hz}, \mathrm{Har}), 7.29(1 \mathrm{H}, \mathrm{t}, \mathrm{J}=7.6 \mathrm{~Hz}, \mathrm{Har}), 3.91(1 \mathrm{H}$, bs, H16ß), $3.91(2 \mathrm{H}, \mathrm{bs}, \mathrm{H} 31), 2.76(1 \mathrm{H}, \mathrm{dd}, \mathrm{J}=12.8 \mathrm{~Hz}, \mathrm{~J}=4.3 \mathrm{~Hz}, \mathrm{H} 3 \alpha), 2.75\left(1 \mathrm{H}, \mathrm{d}, \mathrm{J}_{\mathrm{gem}}=\right.$ $11.6 \mathrm{~Hz}, \mathrm{H} 29 \mathrm{~b}), 2.71(1 \mathrm{H}, \mathrm{dq} ; \mathrm{J}=11.3 \mathrm{~Hz}, \mathrm{~J}=6.4 \mathrm{~Hz}, \mathrm{H} 20), 2.65\left(1 \mathrm{H}, \mathrm{d}, \mathrm{J}_{\mathrm{gem}}=11.6 \mathrm{~Hz}, \mathrm{H} 29 \mathrm{a}\right)$, $2.51\left(1 \mathrm{H}\right.$, septuplet, $\left.\mathrm{J}=7.0 \mathrm{~Hz}, \mathrm{H} 2^{\prime}\right), 2.45\left(1 \mathrm{H}, \mathrm{d}, \mathrm{J}_{\text {gem }}=17.4 \mathrm{~Hz}, \mathrm{H} 12 \alpha\right), 2.37\left(1 \mathrm{H}, \mathrm{dt}, \mathrm{J}_{\text {gem }}=\right.$ $\left.13.7 \mathrm{~Hz}, \mathrm{~J}_{1 \beta-2}=3.7 \mathrm{~Hz}, \mathrm{H} 1 \beta\right), 2.26\left(1 \mathrm{H}, \mathrm{d}, \mathrm{J}_{\mathrm{gem}}=17.4 \mathrm{~Hz}, \mathrm{H} 12 \beta\right), 2.13\left(3 \mathrm{H}, \mathrm{s}, \mathrm{N}_{\mathrm{B}} \mathrm{Me}\right), 2.00(1 \mathrm{H}$, m, H17 $), 1.98(2 \mathrm{H}, \mathrm{m}, \mathrm{H} 2 \alpha+\mathrm{H} 8 \beta), 1.90(1 \mathrm{H}, \mathrm{dd}, \mathrm{J}=14.0 \mathrm{~Hz}, \mathrm{~J}=10.1 \mathrm{~Hz}, \mathrm{H} 15 \beta), 1.62(1 \mathrm{H}$, $\mathrm{qd}, \mathrm{J}=13.4 \mathrm{~Hz}, \mathrm{~J}=4.0 \mathrm{~Hz}, \mathrm{H} 2 \beta), 1.56\left(1 \mathrm{H}, \mathrm{d}, \mathrm{J}_{\mathrm{gem}}=4.3 \mathrm{~Hz}, \mathrm{H} 19 \alpha\right), 1.49(1 \mathrm{H}, \mathrm{m}, \mathrm{H} 7 \beta), 1.46$ $(1 \mathrm{H}, \mathrm{m}, \mathrm{H} 6 \alpha), 1.39(1 \mathrm{H}, \mathrm{dd}, \mathrm{J}=14.0 \mathrm{~Hz}, \mathrm{~J}=2.7 \mathrm{~Hz}, \mathrm{H} 15 \alpha), 1.34(1 \mathrm{H}, \mathrm{dd}, \mathrm{J}=12.5 \mathrm{~Hz}, \mathrm{~J}=$ $3.7 \mathrm{~Hz}, \mathrm{H} 5 \alpha), 1.27(1 \mathrm{H}, \mathrm{m}, \mathrm{H} 7 \alpha), 1.25(1 \mathrm{H}, \mathrm{m}, \mathrm{H} 1 \alpha), 1.14(3 \mathrm{H}, \mathrm{s}, \mathrm{H} 28), 1.11+1.10(6 \mathrm{H}, 2 \mathrm{~d}, \mathrm{~J}=$ $7.0 \mathrm{~Hz}, \mathrm{H} 3{ }^{\prime}+\mathrm{H} 4$ '), $1.03\left(1 \mathrm{H}, \mathrm{d}, \mathrm{J}_{\text {gem }}=4.3 \mathrm{~Hz}, \mathrm{H} 19 \beta\right), 0.95(1 \mathrm{H}, \mathrm{m}, \mathrm{H} 6 \beta), 0.93\left(3 \mathrm{H}, \mathrm{d}, \mathrm{J}_{21-20}=\right.$ $6.7 \mathrm{~Hz}, \mathrm{H} 21), 0.69(3 \mathrm{H}, \mathrm{s}, \mathrm{H} 18), 0.69(3 \mathrm{H}, \mathrm{s}, \mathrm{H} 30) ;{ }^{13} \mathrm{C} \mathrm{NMR}\left(75.4 \mathrm{MHz}, \mathrm{CD}_{3} \mathrm{CN}\right): \delta(\mathrm{ppm})$ 211.4 (C11), 165.8 (C1'), 137.7 (C32), 131.9 (Car), 127.2 (Car), 77.8 (C16), 62.7 (C3), 59.7 (C20), 56.1 (C31+C17), 51.5 (C12), 49.8 (C5), 47.2 (C14), 44.9 (C13), 42.8 (C15), 41.4 (C8), 40.3 (C2'), 38.8 (C29), 38.5 (C10), 34.4 ( $\left.\mathrm{N}_{\mathrm{B}} \mathrm{Me}+\mathrm{C} 9\right), 33.4$ (C4), 30.6 (C2), 30.3 (C19), 28.0 (C1), 24.4 (C7), 21.0+20.8 (C3'+C4'), 20.5 (C28), 18.0 (C6), 17.8 (C18), 11.3 (C30), 10.9 (C21), MS (ESI, m/z) : $645.4(\mathrm{M}+\mathrm{H})$; HRMS (ESI, m/z) : calcd for $\mathrm{C}_{37} \mathrm{H}_{52} \mathrm{~N}_{2} \mathrm{O}_{2} \mathrm{SF}_{3}: 645.3702$, found : 645.3704 . 


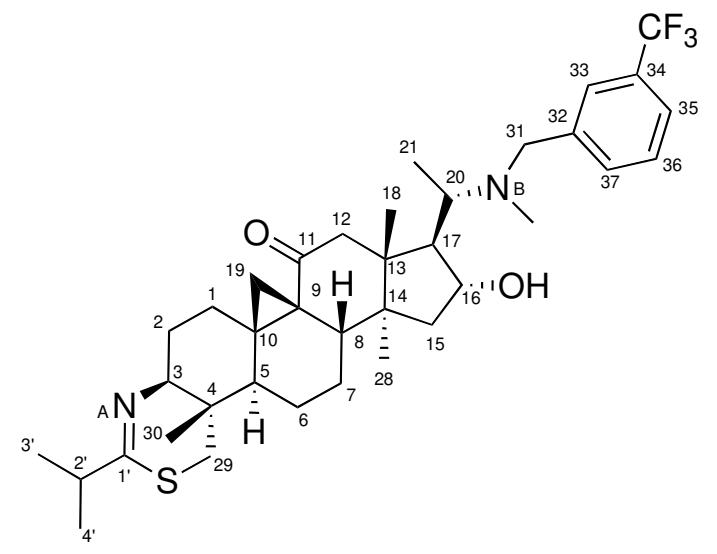

$16 \mathrm{mg}(0,031 \mathrm{mmol}, 1$ eq. $)$ of $\mathbf{3 4}, 13 \mathrm{mg}(0.093 \mathrm{mmol}, 3$ eq. $)$ of potassium carbonate, $10 \mathrm{mg}(0.040 \mathrm{mmol}, 1.3 \mathrm{eq}$.) of 3-trifluoromethylbenzyl bromide and $0.5 \mathrm{~mL}$ of DMF were used by following similar procedure described for the preparation of 36e to give the title compound as an amorphous white solid (13 mg, yield: $62 \%)$. IR $\left(\mathrm{cm}^{-1}\right): 3358,2955,2928,2854,1666,1632$, 1450, 1327, 1163, 1224, $1072 ;{ }^{1} \mathrm{H}$ NMR $\left(500 \mathrm{MHz}, \mathrm{CD}_{3} \mathrm{CN}, 333 \mathrm{~K}\right): \delta(\mathrm{ppm}) 7.52(3 \mathrm{H}, \mathrm{m}$, $\mathrm{H} 33+\mathrm{H} 35+\mathrm{H} 37), 7.46(1 \mathrm{H}, \mathrm{t}, \mathrm{J}=7.9 \mathrm{~Hz}, \mathrm{H} 36), 4.01(1 \mathrm{H}, \mathrm{bs}, \mathrm{H} 16 \beta), 3.67(1 \mathrm{H}, \mathrm{bs}, \mathrm{H} 31), 2.84$ $(1 \mathrm{H}, \mathrm{dd}, \mathrm{J}=12.8 \mathrm{~Hz}, \mathrm{~J}=4.3 \mathrm{~Hz}, \mathrm{H} 3 \alpha), 2.83\left(1 \mathrm{H}, \mathrm{d}, \mathrm{J}_{\mathrm{gem}}=11.3 \mathrm{~Hz}, \mathrm{H} 29 \mathrm{~b}\right), 2.79(1 \mathrm{H}, \mathrm{dq}, \mathrm{J}=$ $11.0 \mathrm{~Hz}, \mathrm{~J}=6.7 \mathrm{~Hz}, \mathrm{H} 20), 2.73\left(1 \mathrm{H}, \mathrm{d}, \mathrm{J}_{\mathrm{gem}}=11.3 \mathrm{~Hz}, \mathrm{H} 29 \mathrm{a}\right), 2.58(1 \mathrm{H}$, septuplet, J = $7.0 \mathrm{~Hz}$, H2'), $2.5\left(1 \mathrm{H}, \mathrm{d}, \mathrm{J}_{\mathrm{gem}}=17.1 \mathrm{~Hz}, \mathrm{H} 12 \alpha\right), 2.44\left(1 \mathrm{H}, \mathrm{dt}, \mathrm{J}_{\mathrm{gem}}=13.4 \mathrm{~Hz}, \mathrm{~J}_{1 \beta-2}=3.4 \mathrm{~Hz}, \mathrm{H} 1 \beta\right), 2.32$ $\left(1 \mathrm{H}, \mathrm{d}, \mathrm{J}_{\mathrm{gem}}=17.1 \mathrm{~Hz}, \mathrm{H} 12 \beta\right), 2.18\left(3 \mathrm{H}, \mathrm{s}, \mathrm{N}_{\mathrm{B}} \mathrm{Me}\right), 2.08(1 \mathrm{H}, \mathrm{m}, \mathrm{H} 17 \alpha), 2.05(2 \mathrm{H}, \mathrm{m}$, $\mathrm{H} 2 \alpha+\mathrm{H} 8 \beta), 1.99(1 \mathrm{H}, \mathrm{m}, \mathrm{H} 15 \beta), 1.70(1 \mathrm{H}, \mathrm{m}, \mathrm{H} 2 \beta), 1.63\left(1 \mathrm{H}, \mathrm{d}, \mathrm{J}_{\mathrm{gem}}=4.0 \mathrm{~Hz}, \mathrm{H} 19 \alpha\right), 1.56$ $(1 \mathrm{H}, \mathrm{m}, \mathrm{H} 7 \beta), 1.53(1 \mathrm{H}, \mathrm{m}, \mathrm{H} 6 \alpha), 1.49(1 \mathrm{H}, \mathrm{m}, \mathrm{H} 15 \alpha), 1.41(1 \mathrm{H}, \mathrm{m}, \mathrm{H} 5 \alpha), 1.32(2 \mathrm{H}, \mathrm{m}$, $\mathrm{H} 1 \alpha+\mathrm{H} 7 \alpha), 1.21(3 \mathrm{H}, \mathrm{s}, \mathrm{H} 28), 1.18+1.16\left(6 \mathrm{H}, 2 \mathrm{~d}, \mathrm{~J}=7.0 \mathrm{~Hz}, \mathrm{H} 3^{\prime}+\mathrm{H} 4{ }^{\prime}\right), 1.13\left(1 \mathrm{H}, \mathrm{J}_{\mathrm{gem}}=4.0 \mathrm{~Hz}\right.$, H19ß), $1.02(1 \mathrm{H}, \mathrm{m}, \mathrm{H} 6 \beta), 0.99\left(3 \mathrm{H}, \mathrm{d}, \mathrm{J}_{21-20}=6.7 \mathrm{~Hz}, \mathrm{H} 21\right), 0.77(3 \mathrm{H}, \mathrm{s}, \mathrm{H} 18), 0.76(3 \mathrm{H}, \mathrm{s}$, H30); ${ }^{13} \mathrm{C}$ NMR (75.4 MHz, $\mathrm{CD}_{3} \mathrm{CN}$ ): $\delta(\mathrm{ppm}) 210.7$ (C11), 165.9 (C1'), 139.7 (C32), 132.3 (C37), 129.1 (C36), 125.8 (C33), 124.2 (C35), 78.0 (C16), 62.7 (C3), 60.7 (C31), 59.7 (C20), 55.9 (C17), 51.4 (C12), 49.8 (C5), 47.2 (C14), 44.8 (C13), 42.8 (C15), 41.4 (C8), 40.3 (C2'), 38.7 (C29), 38.5 (C10), $34.4\left(\mathrm{~N}_{\mathrm{B}} \mathrm{Me}+\mathrm{C} 9\right), 33.4$ (C4), 30.6 (C2), 30.4 (C19), 28.0 (C1), 24.4 (C7), 21.0+20.8 (C3'+C4'), 20.6 (C28), 18.0 (C6), 17.7 (C18), 11.3 (C30), 10.8 (C21); MS (ESI, $\mathrm{m} / \mathrm{z}): 645.4(\mathrm{M}+\mathrm{H})$; HRMS (ESI, m/z) : calcd for $\mathrm{C}_{37} \mathrm{H}_{52} \mathrm{~N}_{2} \mathrm{O}_{2} \mathrm{SF}_{3}: 645.3702$, found : 645.3708. 


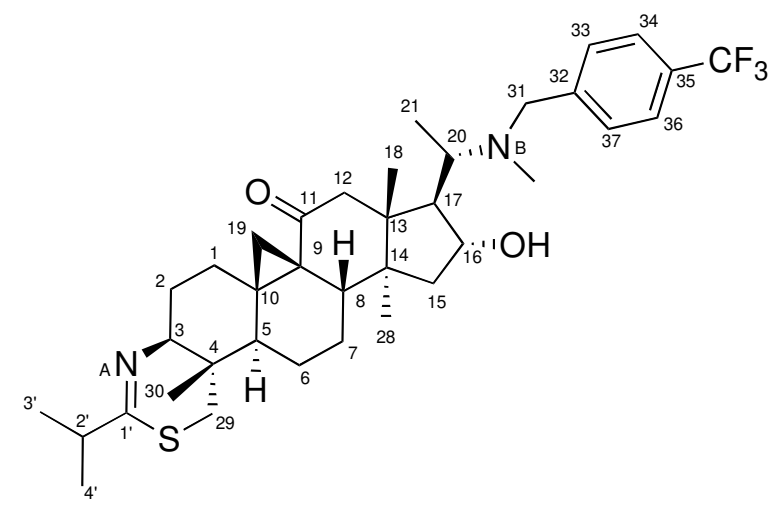

$16 \mathrm{mg}(0,031 \mathrm{mmol}, 1$ eq. $)$ of $\mathbf{3 4}, 13 \mathrm{mg}(0.093 \mathrm{mmol}, 3$ eq. $)$ of potassium carbonate, $10 \mathrm{mg}(0.040 \mathrm{mmol}, 1.3 \mathrm{eq}$.) of 4-trifluoromethylbenzyl bromide and $0.5 \mathrm{~mL}$ of DMF were used by following similar procedure described for the preparation of 36e to give the title compound as an amorphous white solid (12 mg, yield: $57 \%)$. IR $\left(\mathrm{cm}^{-1}\right): 3361,2968,2929,2866,1666,1636$, 1617, 1456, 1323, 1161, 1122, 1065, $1017 .{ }^{1} \mathrm{H}$ NMR (500 MHz, $\left.\mathrm{CD}_{3} \mathrm{CN}, 333 \mathrm{~K}\right): \delta(\mathrm{ppm}) 7.61$ $(2 \mathrm{H}, \mathrm{d}, \mathrm{J}=8.2 \mathrm{~Hz}, \mathrm{H} 34+\mathrm{H} 36), 7.44(2 \mathrm{H}, \mathrm{d}, \mathrm{J}=8.2 \mathrm{~Hz}, \mathrm{H} 33+\mathrm{H} 37), 4.04(1 \mathrm{H}, \mathrm{bs}, \mathrm{H} 16 \beta), 3.69$ $(2 \mathrm{H}, \mathrm{bs}, \mathrm{H} 31), 2.86(1 \mathrm{H}, \mathrm{dd}, \mathrm{J}=12.8 \mathrm{~Hz}, \mathrm{~J}=4.0 \mathrm{~Hz}, \mathrm{H} 3 \alpha), 2.85\left(1 \mathrm{H}, \mathrm{d}, \mathrm{J}_{\text {gem }}=11.6 \mathrm{~Hz}, \mathrm{H} 29 \mathrm{~b}\right)$, $2.81(1 \mathrm{H}, \mathrm{m}, \mathrm{H} 20), 2.75\left(1 \mathrm{H}, \mathrm{d}, \mathrm{J}_{\mathrm{gem}}=11.6 \mathrm{~Hz}, \mathrm{H} 29 \mathrm{a}\right), 2.61(1 \mathrm{H}$, septuplet, J = 7.0 Hz, H2'), $2.54\left(1 \mathrm{H}, \mathrm{d}, \mathrm{J}_{\mathrm{gem}}=17.1 \mathrm{~Hz}, \mathrm{H} 12 \alpha\right), 2.46\left(1 \mathrm{H}, \mathrm{dt}, \mathrm{J}_{\mathrm{gem}}=13.7 \mathrm{~Hz}, \mathrm{~J}_{1 \beta-2}=3.5 \mathrm{~Hz}, \mathrm{H} 1 \beta\right), 2.33(1 \mathrm{H}$, d, $\left.\mathrm{J}_{\mathrm{gem}}=17.1 \mathrm{~Hz}, \mathrm{H} 12 \beta\right), 2.19\left(3 \mathrm{H}, \mathrm{s}, \mathrm{N}_{\mathrm{B}} \mathrm{Me}\right), 2.11(2 \mathrm{H}, \mathrm{m}, \mathrm{H} 8 \beta+\mathrm{H} 17 \alpha), 2.07(1 \mathrm{H}, \mathrm{m}, \mathrm{H} 2 \alpha)$, $2.01(1 \mathrm{H}, \mathrm{m}, \mathrm{H} 15 \beta), 1.71(1 \mathrm{H}, \mathrm{m}, \mathrm{H} 2 \beta), 1.65\left(1 \mathrm{H}, \mathrm{d}, \mathrm{J}_{\mathrm{gem}}=4.0 \mathrm{~Hz}, \mathrm{H} 19 \alpha\right), 1.59(1 \mathrm{H}, \mathrm{m}, \mathrm{H} 7 \beta)$, $1.55(1 \mathrm{H}, \mathrm{m}, \mathrm{H} 6 \alpha), 1.51(1 \mathrm{H}, \mathrm{m}, \mathrm{H} 15 \alpha), 1.44(1 \mathrm{H}, \mathrm{m}, \mathrm{H} 5 \alpha), 1.32(2 \mathrm{H}, \mathrm{m}, \mathrm{H} 1 \alpha+\mathrm{H} 7 \alpha), 1.23(3 \mathrm{H}$, s, H28), 1.20+1.18 (6H, 2d, J = 7.0 Hz, H3'+H4'), $1.13(1 \mathrm{H}, \mathrm{d}, \mathrm{J}=4.0 \mathrm{~Hz}, \mathrm{H} 19 \beta), 1.00$ (3H, d, $\left.\mathrm{J}_{21-20}=6.7 \mathrm{~Hz}, \mathrm{H} 21\right), 0.88(1 \mathrm{H}, \mathrm{m}, \mathrm{H} 6 \beta), 0.79(3 \mathrm{H}, \mathrm{s}, \mathrm{H} 18), 0.77(3 \mathrm{H}, \mathrm{s}, \mathrm{H} 30) ;{ }^{13} \mathrm{C}$ NMR $(75.4$ $\left.\mathrm{MHz}, \mathrm{CD}_{3} \mathrm{CN}\right): \delta(\mathrm{ppm}) 210.7(\mathrm{C} 11), 165.8\left(\mathrm{Cl}^{\prime}\right), 142.8(\mathrm{C} 32), 129.3(\mathrm{C} 33+\mathrm{C} 37), 125.5$ (C34+C36), 78.0 (C16), 62.6 (C3), 60.8 (C31), 60.0 (C20), 55.9 (C17), 51.4 (C12), 49.8 (C5), 47.2 (C14), 44.8 (C13), 42.7 (C15), 41.3 (C8), 40.3 (C2'), 38.7 (C29), 38.5 (C10), 34.4 $\left(\mathrm{N}_{\mathrm{B}} \mathrm{Me}+\mathrm{C} 9\right), 33.4(\mathrm{C} 4), 30.5(\mathrm{C} 2), 30.4(\mathrm{C} 19), 28.0(\mathrm{C} 1), 24.4(\mathrm{C} 7), 21.1+20.8\left(\mathrm{C}^{\prime}{ }^{\circ}+\mathrm{C} 4{ }^{\prime}\right), 20.6$ (C28), 18.0 (C6), 17.8 (C18), 11.3 (C30), 10.8 (C21); MS (ESI, m/z) : 645.4 (M+H); HRMS (ESI, m/z) : calcd for $\mathrm{C}_{37} \mathrm{H}_{52} \mathrm{~N}_{2} \mathrm{O}_{2} \mathrm{SF}_{3}: 645.3702$, found : 645.3728 . 


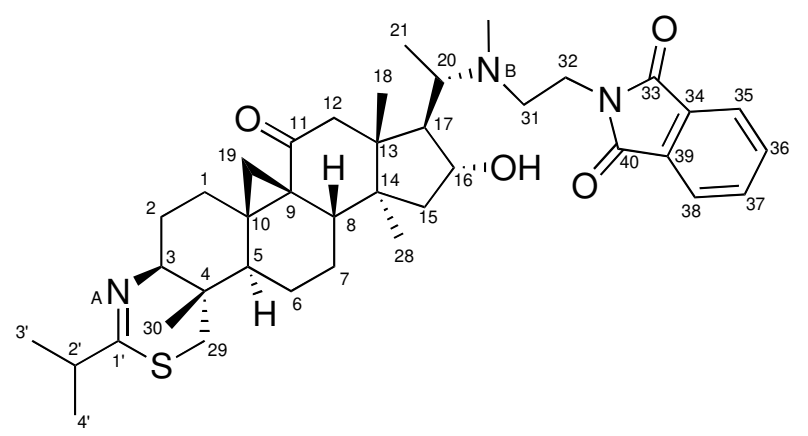

$15.7 \mathrm{mg} \quad(0.032 \mathrm{mmol}, \quad 1$ eq. $) \quad$ of $\quad \mathbf{3 4}, \quad 24 \mathrm{mg} \quad(0.097 \mathrm{mmol}, \quad 3$ eq. $)$ of $2-(2-$ bromoethyl)isoindole-1,3-dione, $13 \mathrm{mg}(0.097 \mathrm{mmol}, 3$ eq. $)$ of potassium carbonate and $1 \mathrm{~mL}$ of acetonitrile were used by following similar procedure described for the preparation of 36a to give the title compound as an amorphous white solid $(15.4 \mathrm{mg}$, yield: $72 \%)$. For this reaction, the mixture was refluxed for $12 \mathrm{~h}$ to give the desired product. IR $\left(\mathrm{cm}^{-1}\right): 3464,2962,2930$, 2873, 1772, 1711, 1668, 1633, 1394; ${ }^{1} \mathrm{H}$ NMR (500 MHz, $\left.\mathrm{CD}_{3} \mathrm{CN}, 333 \mathrm{~K}\right): \delta(\mathrm{ppm}) 7.80(2 \mathrm{H}, \mathrm{m}$, $\mathrm{H} 35+\mathrm{H} 38), 7.76(2 \mathrm{H}, \mathrm{m}, \mathrm{H} 36+\mathrm{H} 37), 3.87(1 \mathrm{H}, \mathrm{m}, \mathrm{H} 16 \beta), 3.80(2 \mathrm{H}, \mathrm{m}, \mathrm{H} 32), 3.70(2 \mathrm{H}, \mathrm{m}$, $\mathrm{H} 31), 2.85\left(1 \mathrm{H}, \mathrm{d}, \mathrm{J}_{\text {gem }}=11.9 \mathrm{~Hz}, \mathrm{H} 29 \mathrm{~b}\right), 2.82(1 \mathrm{H}, \mathrm{dd}, \mathrm{J}=12.8 \mathrm{~Hz}, \mathrm{~J}=4.0 \mathrm{~Hz}, \mathrm{H} 3 \alpha), 2.75(1 \mathrm{H}$, d, $\left.\mathrm{J}_{\mathrm{gem}}=11.9 \mathrm{~Hz}, \mathrm{H} 29 \mathrm{a}\right), 2.70(1 \mathrm{H}, \mathrm{m}, \mathrm{H} 20), 2.51\left(1 \mathrm{H}, \mathrm{d}, \mathrm{J}_{\mathrm{gem}}=17.1 \mathrm{~Hz}, \mathrm{H} 12 \alpha\right), 2.46(1 \mathrm{H}, \mathrm{m}$, H2'), $2.31(1 \mathrm{H}, \mathrm{m}, \mathrm{H} 1 \beta), 2.30\left(3 \mathrm{H}, \mathrm{s}, \mathrm{N}_{\mathrm{B}} \mathrm{Me}\right), 2.19\left(1 \mathrm{H}, \mathrm{d}, \mathrm{J}_{\mathrm{gem}}=17.1 \mathrm{~Hz}, \mathrm{H} 12 \beta\right), 2.04(1 \mathrm{H}, \mathrm{dd}$, $\mathrm{J}=10.4 \mathrm{~Hz}, \mathrm{~J}=7.0 \mathrm{~Hz}, \mathrm{H} 8 \beta), 1.98(1 \mathrm{H}, \mathrm{dd}, \mathrm{J}=10.7 \mathrm{~Hz}, \mathrm{~J}=7.0 \mathrm{~Hz}, \mathrm{H} 17 \alpha), 1.93(1 \mathrm{H}, \mathrm{m}, \mathrm{H} 2 \alpha)$, $1.84(1 \mathrm{H}, \mathrm{dd}, \mathrm{J}=14.0 \mathrm{~Hz}, \mathrm{~J}=10.4 \mathrm{~Hz}, \mathrm{H} 15 \beta), 1.60(1 \mathrm{H}, \mathrm{m}, \mathrm{H} 2 \beta), 1.48(3 \mathrm{H}, \mathrm{m}$, $\mathrm{H} 6 \alpha+\mathrm{H} 7 \beta+\mathrm{H} 19 \alpha), 1.44(1 \mathrm{H}, \mathrm{m}, \mathrm{H} 5 \alpha), 1.29(1 \mathrm{H}, \mathrm{m}, \mathrm{H} 1 \alpha), 1.27(1 \mathrm{H}, \mathrm{m}, \mathrm{H} 7 \alpha), 1.20(1 \mathrm{H}, \mathrm{m}$, $\mathrm{H} 15 \alpha), 1.11\left(10 \mathrm{H}, \mathrm{m}, \mathrm{H} 3^{\prime}+\mathrm{H}^{\prime}{ }^{\prime}+\mathrm{H} 19 \beta+\mathrm{H} 28\right), 1.03(1 \mathrm{H}, \mathrm{m}, \mathrm{H} 6 \beta), 0.91\left(3 \mathrm{H}, \mathrm{d}, \mathrm{J}_{21-20}=6.4 \mathrm{~Hz}\right.$, $\mathrm{H} 21), 0.78$ (3H, s, H18), $0.72(3 \mathrm{H}, \mathrm{s}, \mathrm{H} 30) .{ }^{13} \mathrm{C}$ NMR (75.4 MHz, CD $\left.{ }_{3} \mathrm{CN}\right): \delta(\mathrm{ppm}) 211.3(\mathrm{C} 11)$, 169.6 (C33+C40), 135.1 (C36+C37), 133.2 (C34+C39), 123.8 (C35+C38), 78.4 (C16), 63.5 (C3+C20), 56.9 (C17), 52.2 (C12), 50.2 (C5), 47.9 (C14), 45.7 (C13), 43.9 (C15), 42.0 (C8), 40.5 (C2'), 39.4 (C29), 38.8 (C10), 37.0 (C32), 35.1 (C9), 34.0 (C4), 31.4 (C2), 30.5 (C19), 28.7 (C1), 25.1 (C7), 21.3+21.2 (C3'+C4'), 20.7 (C28), 18.7 (C6), 18.1 (C18), 11.5 (C21+C30); MS $\left(\right.$ ESI, m/z) : $660.3(\mathrm{M}+\mathrm{H})$; HRMS (ESI, m/z) : calcd for $\mathrm{C}_{39} \mathrm{H}_{54} \mathrm{~N}_{3} \mathrm{O}_{4} \mathrm{~S}: 660.3835$, found : 660.3817 . 


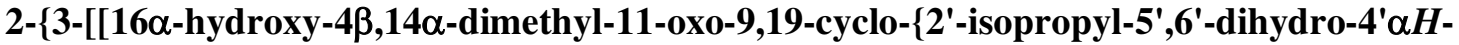 $\left[1^{\prime}, 3^{\prime}\right]$ thiazino[4',5':3,4]\}-5 $\alpha, 9 \beta$-pregnan-20S-yl](methyl)amino]propyl $\}-1 H$-isoindole- 1,3(2H)-dione (36k)}

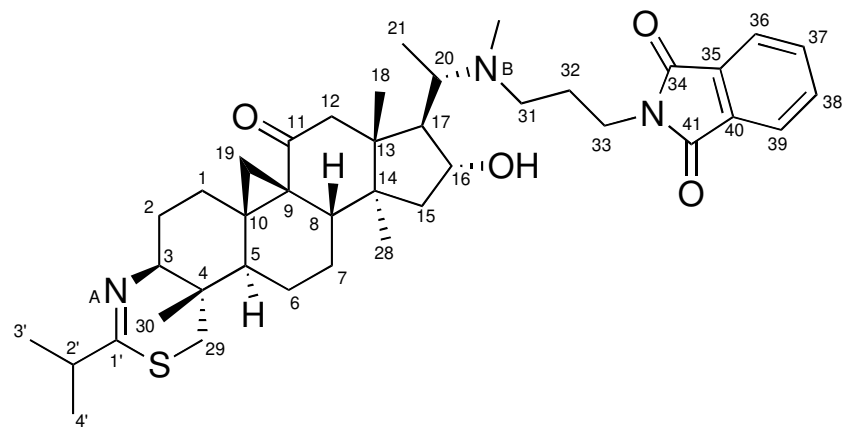

$11.3 \mathrm{mg} \quad(0.023 \mathrm{mmol}, \quad 1$ eq. $)$ of $\mathbf{3 4}, \quad 9.3 \mathrm{mg} \quad(0.035 \mathrm{mmol}, \quad 1.5$ eq. $)$ of 2-(3bromopropyl)isoindole-1,3-dione, $10 \mathrm{mg}(0.070 \mathrm{mmol}, 3$ eq. $)$ of potassium carbonate and $1 \mathrm{~mL}$ of acetonitrile were used by following similar procedure described for the preparation of 36a75\%) to give the title compound as an amorphous white solid (11.7 mg, yield: $75 \%)$. For this reaction, the mixture was refluxed for $30 \mathrm{~h}$ to give the desired product. IR $\left(\mathrm{cm}^{-1}\right): 2960$, 2928, 2866, 1770, 1711, 1669, 1635, 1395; ${ }^{1} \mathrm{H}$ NMR (500 MHz, $\left.\mathrm{CD}_{3} \mathrm{CN}, 333 \mathrm{~K}\right): \delta(\mathrm{ppm}) 7.81$ $(2 \mathrm{H}, \mathrm{m}, \mathrm{H} 36+\mathrm{H} 39), 7.76(2 \mathrm{H}, \mathrm{m}, \mathrm{H} 37+\mathrm{H} 38), 4.20(1 \mathrm{H}, \mathrm{m}, \mathrm{H} 16 \beta), 3.69(2 \mathrm{H}, \mathrm{m}, \mathrm{H} 33), 2.86(1 \mathrm{H}$, $\left.\mathrm{d}, \mathrm{J}_{\mathrm{gem}}=11.6 \mathrm{~Hz}, \mathrm{H} 29 \mathrm{~b}\right), 2.82(2 \mathrm{H}, \mathrm{m}, \mathrm{H} 3 \alpha+\mathrm{H} 20), 2.75\left(1 \mathrm{H}, \mathrm{d}, \mathrm{J}_{\text {gem }}=11.6 \mathrm{~Hz}, \mathrm{H} 29 \mathrm{a}\right), 2.61(2 \mathrm{H}$, m, H31), $2.53\left(1 \mathrm{H}, \mathrm{d}, \mathrm{J}_{\mathrm{gem}}=17.1 \mathrm{~Hz}, \mathrm{H} 12 \alpha\right), 2.48(1 \mathrm{H}, \mathrm{sept}, \mathrm{J}=6.7 \mathrm{~Hz}, \mathrm{H} 2$ '), $2.35(1 \mathrm{H}, \mathrm{dm}, \mathrm{J}=$ $13.4 \mathrm{~Hz}, \mathrm{H} 1 \beta), 2.28\left(3 \mathrm{H}, \mathrm{s}, \mathrm{N}_{\mathrm{B}} \mathrm{Me}\right), 2.23\left(1 \mathrm{H}, \mathrm{d}, \mathrm{J}_{\mathrm{gem}}=17.1 \mathrm{~Hz}, \mathrm{H} 12 \beta\right), 2.09(2 \mathrm{H}, \mathrm{m}$, $\mathrm{H} 8 \beta+\mathrm{H} 17 \alpha), 2.02(1 \mathrm{H}, \mathrm{m}, \mathrm{H} 15 \beta), 1.95(1 \mathrm{H}, \mathrm{m}, \mathrm{H} 2 \alpha), 1.89$ (1H, m, H32), $1.64(1 \mathrm{H}, \mathrm{m}, \mathrm{H} 2 \beta)$, $1.52(3 \mathrm{H}, \mathrm{m}, \mathrm{H} 6 \alpha+\mathrm{H} 7 \beta+\mathrm{H} 19 \alpha), 1.46(1 \mathrm{H}, \mathrm{dd}, \mathrm{J}=12.2 \mathrm{~Hz}, \mathrm{~J}=3.4 \mathrm{~Hz}, \mathrm{H} 5 \alpha), 1.40(1 \mathrm{H}, \mathrm{dd}, \mathrm{J}=$ $14.0 \mathrm{~Hz}, \mathrm{~J}=2.4 \mathrm{~Hz}, \mathrm{H} 15 \alpha), 1.30(2 \mathrm{H}, \mathrm{m}, \mathrm{H} 1 \alpha+\mathrm{H} 7 \alpha), 1.17(3 \mathrm{H}, \mathrm{s}, \mathrm{H} 28), 1.13(7 \mathrm{H}, \mathrm{m}$, H3'+H4'+H19ß), $1.06(1 \mathrm{H}, \mathrm{m}, \mathrm{H} 6 \beta), 0.93\left(3 \mathrm{H}, \mathrm{d}, \mathrm{J}_{21-20}=6.4 \mathrm{~Hz}, \mathrm{H} 21\right), 0.86$ (3H, s, H18), 0.74 $(3 \mathrm{H}, \mathrm{s}, \mathrm{H} 30) ;{ }^{13} \mathrm{C}$ NMR $\left(75.4 \mathrm{MHz}, \mathrm{CD}_{3} \mathrm{CN}\right): \delta(\mathrm{ppm}) 211.1(\mathrm{C} 11), 169.1$ (C34+C41), 134.8 (C37+C38), 132.9 (C35+C40), 123.6 (C36+C39), 77.4 (C16), 63.3 (C3+C20), 56.6 (C17), 52.1 (C12), 50.1 (C5), 47.8 (C14), 45.6 (C13), 43.9 (C15), 41.9 (C8), 40.4 (C2'), 39.3 (C29), 38.7 (C10), 36.4 (C33), 35.0 (C9), 33.8 (C4), 31.2 (C2), 30.2 (C19), 28.5 (C1), 27.2 (C32), 25.0 (C7), 21.3+21.2 (C3'+C4'), 20.7 (C28), 18.5 (C6), 18.2 (C18), 11.5 (C30), 11.2 (C21); MS (ESI, m/z) : $674.4(\mathrm{M}+\mathrm{H})$; HRMS (ESI, m/z) : calcd for $\mathrm{C}_{40} \mathrm{H}_{56} \mathrm{~N}_{3} \mathrm{O}_{4} \mathrm{~S}: 674.3992$, found : 674.3975. 


\section{Purity of tested compounds}

The purity of tested compounds were mesured using reversed-phase UHPLC (HSS C$18,2.1 \times 50 \mathrm{~mm} \mathrm{~s}-1.8 \mu \mathrm{M})$. All compounds for which biological data is reported are $>95 \%$ purity. Compounds were eluted with $95 / 5 \mathrm{~A} / \mathrm{B}$ for 0.3 min then with a gradient of $5-100 \% \mathrm{~B} / \mathrm{A}$ for $4.2 \mathrm{~min}$ followed by $0 / 100 \mathrm{~A} / \mathrm{B}$ isocratic for $0.5 \mathrm{~min}$ at flow rate of $0.6 \mathrm{ml} / \mathrm{min}$, where solvent $\mathrm{A}$ was $0.1 \%$ formic acid in $\mathrm{H}_{2} \mathrm{O}$ and solvent $\mathrm{B}$ was $0.1 \%$ formic acid in MeCN. Purity was determined from the absorbance at $230 \mathrm{~nm}$.

\section{TABLE LISTING THE DEGREE OF PURITY FOR TESTED COMPOUNDS (percent and retention time)}

\begin{tabular}{|c|c|}
\hline Compound & purity \\
\hline $9 a$ & $>98 \%(2.16 \mathrm{~min})$ \\
\hline 9b & $>96 \%(2.21 \mathrm{~min})$ \\
\hline $9 \mathrm{c}$ & $>95 \%(2.18 \mathrm{~min})$ \\
\hline 13a & $>95 \%(1.73 \mathrm{~min})$ \\
\hline 13b & $>95 \%(1.44 \mathrm{~min})$ \\
\hline 20 & $>99 \%(1.70 \mathrm{~min})$ \\
\hline 25 & $>95 \%(2.86 \mathrm{~min})$ \\
\hline 27 & $>95 \%(2.96 \mathrm{~min})$ \\
\hline 30 & $>97 \%(1.35 \mathrm{~min})$ \\
\hline 34 & $>95 \%(1.39 \mathrm{~min})$ \\
\hline $35 \mathbf{a}$ & $>98 \%(1.12 \mathrm{~min})$ \\
\hline $35 \mathrm{~b}$ & $>95 \%(2.75 \mathrm{~min})$ \\
\hline $35 \mathrm{c}$ & $>95 \%(3.08 \mathrm{~min})$ \\
\hline $36 \mathbf{a}$ & $>95 \%(1.42 \mathrm{~min})$ \\
\hline $36 \mathbf{b}$ & $>95 \%(1.74 \mathrm{~min})$ \\
\hline $36 c$ & $>95 \%(1.78 \mathrm{~min})$ \\
\hline 36d & $>95 \%(1.75 \mathrm{~min})$ \\
\hline $36 \mathbf{e}$ & $>95 \%(1.75 \mathrm{~min})$ \\
\hline 36f & $>98 \%(1.71 \mathrm{~min})$ \\
\hline $36 \mathrm{~g}$ & $>95 \%(1.81 \mathrm{~min})$ \\
\hline 36h & $>95 \%(1.87 \mathrm{~min})$ \\
\hline $36 \mathbf{i}$ & $>95 \%(1.87 \mathrm{~min})$ \\
\hline $36 j$ & $>95 \%(1.68 \mathrm{~min})$ \\
\hline $36 \mathbf{k}$ & $>96 \%(1.73 \mathrm{~min})$ \\
\hline
\end{tabular}




\section{Computer-Aided Molecular Modeling}

\section{RMS Distance}

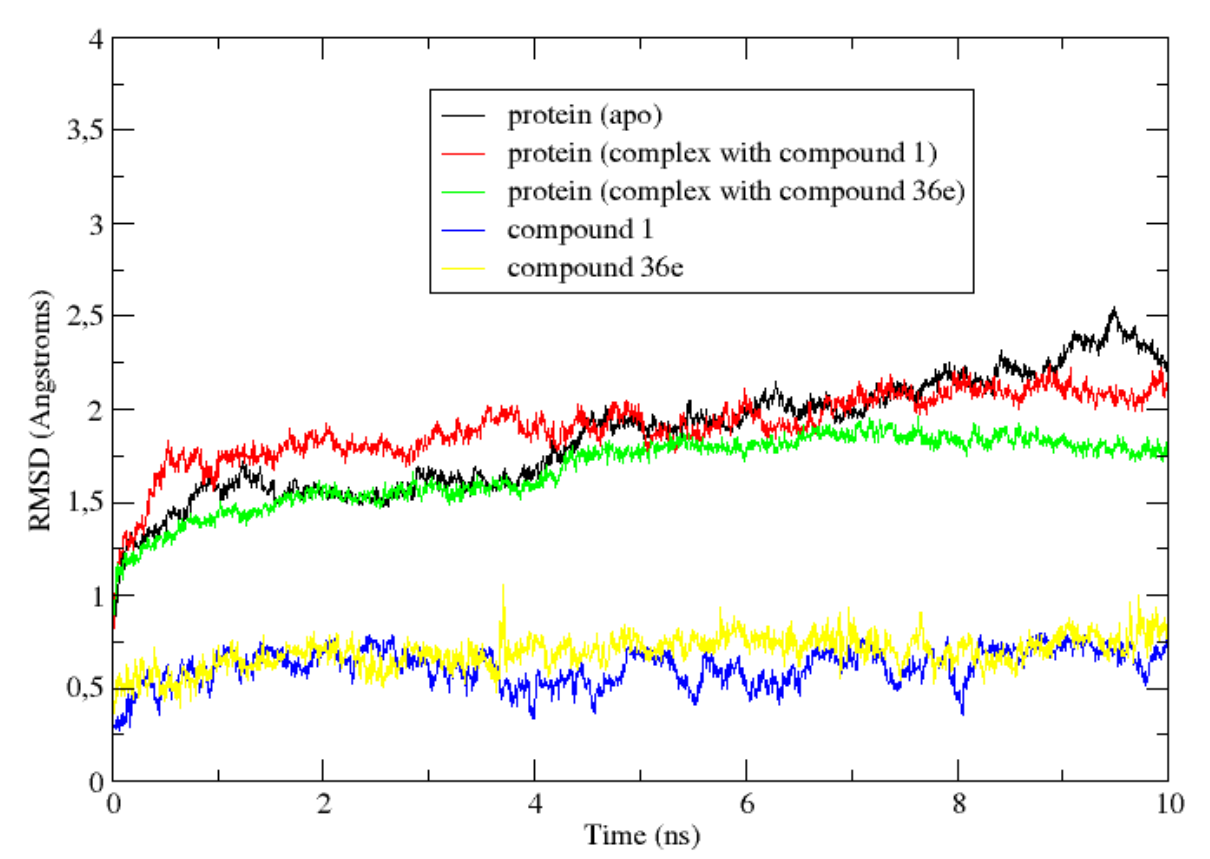

Figure S1. Root mean square deviation (RMSD) for compounds 1 (blue) and 36e (yellow), and for protein in apo form (black) and in complex with compounds 1 (red) and 36e (green), during the $10 \mathrm{~ns}$ molecular dynamics simulations 


\section{RMS fluctuation}

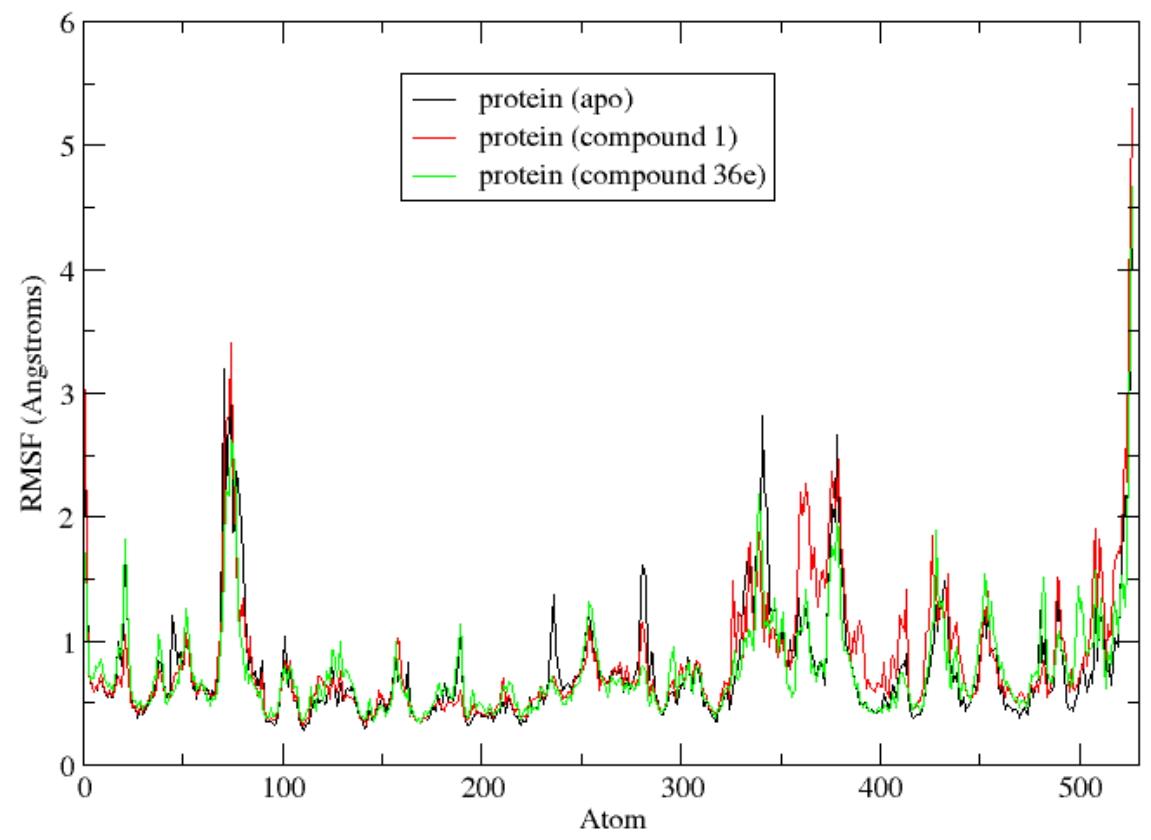

Figure S2. Root mean square fluctuation (RMSF) of CA atoms for protein in apo form (black) and in complex with compounds 1 (red) and 36e (green), during the $10 \mathrm{~ns}$ molecular dynamics simulations 


\section{Contact Surface}

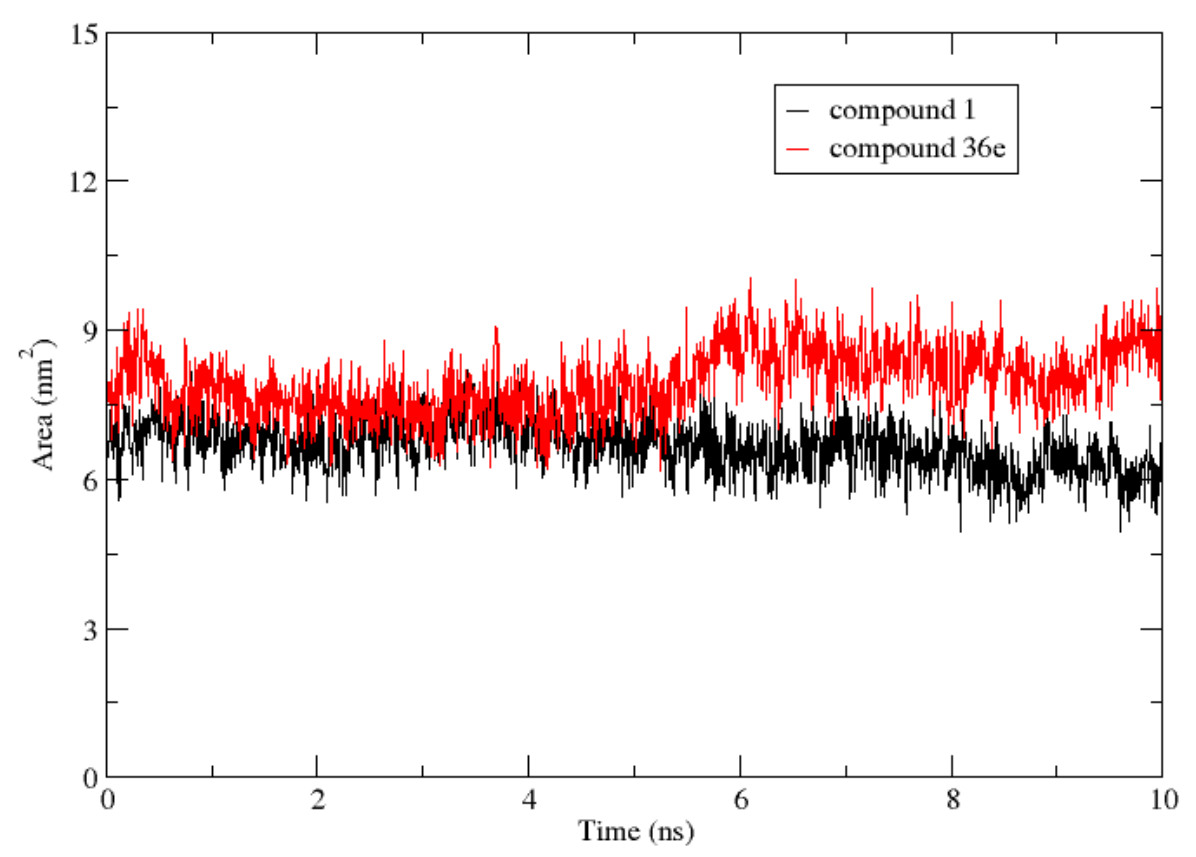

Figure S3. Contact surface between protein and compounds $\mathbf{1}$ (black) and 36e (red), during the $10 \mathrm{~ns}$ molecular dynamics simulations

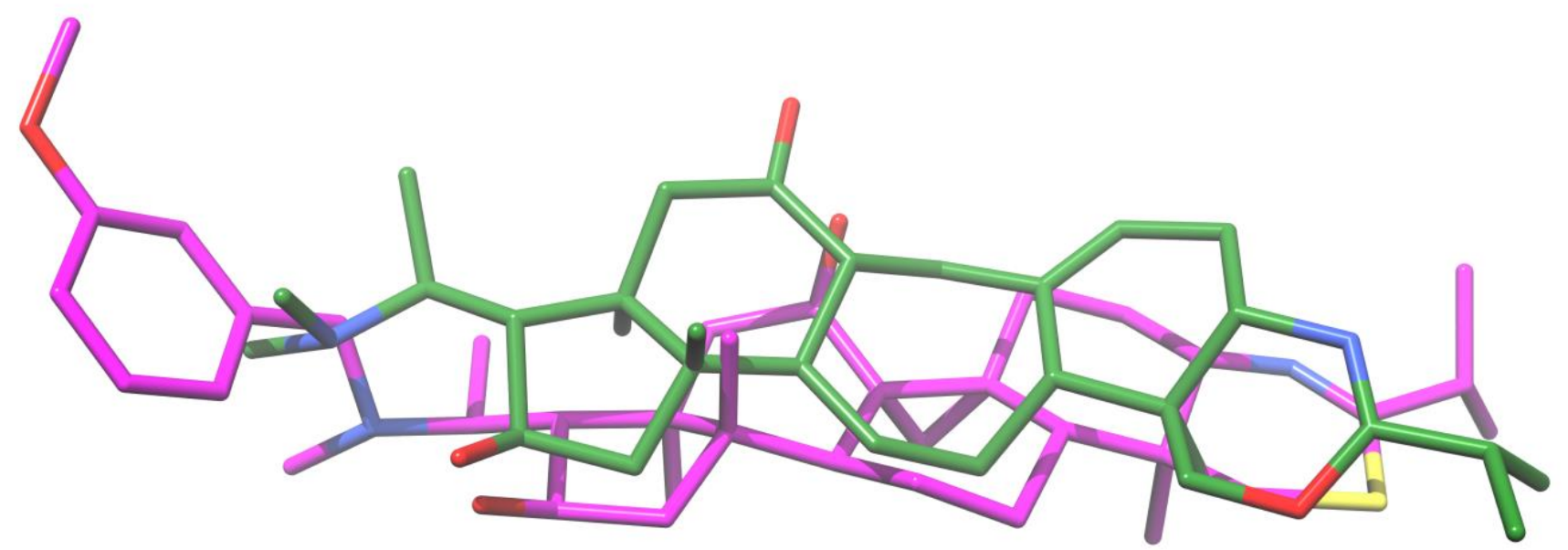

Figure S4. Superposition of equilibrium conformations in the AChE binding site for compounds 1 (green) and 36e (magenta), after $10 \mathrm{~ns}$ molecular dynamics simulations 